volume 8

ISSUE 3

2020 maggio-giugno

Editor in Chief Michele Meschi

Supervisor Editor Roberto Nardi

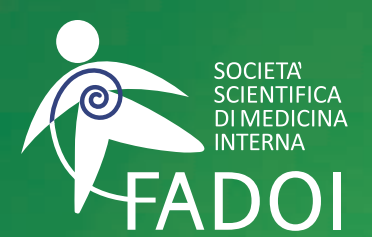

FEDERAZIONE

DELLE ASSOCIAZIONI

DEI DIRIGENTI

OSPEDALIERI

INTERNISTI

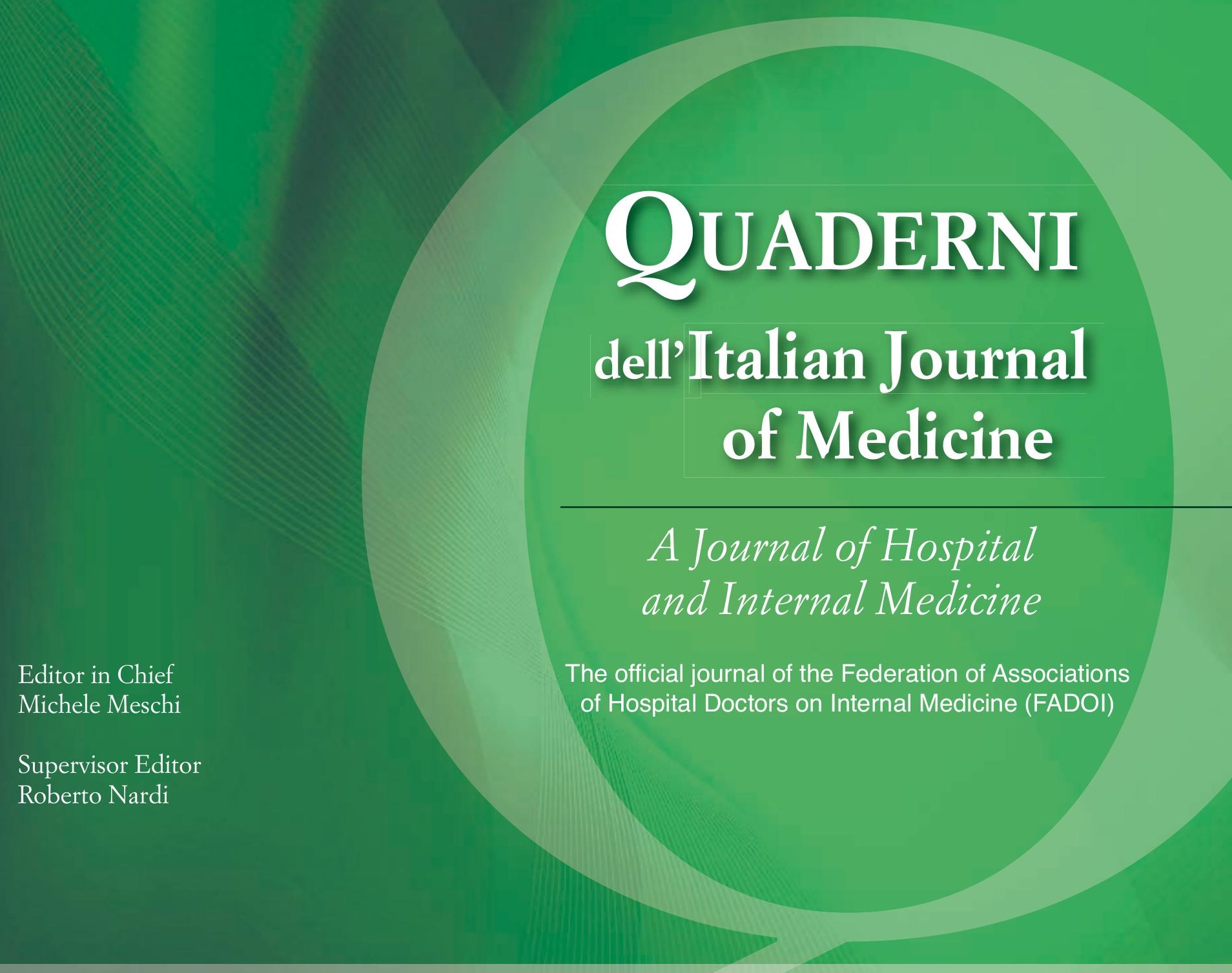

\title{
Un giorno in Medicina. Un viaggio attraverso le opinioni degli Internisti FADOI e degli infermieri ANIMO
}

Guest Editors: Ruggero Pastorelli, Gabriella Bordin 
PRESIDENTE ELETTO

Francesco Dentali, Varese, Italy

\section{PAST PRESIDENT}

Andrea Fontanella, Napoli, Italy

\section{SEGRETARIO}

Paola Gnerre, Savona, Italy

\section{STAFF DI SEGRETERIA}

Maurizia Gambacorta, Todi (PG), Italy

Ada Maffettone, Napoli, Italy

Claudia Tieri, Bari, Italy

\section{TESORIERE}

Giorgio Ballardini, Rimini, Italy

\section{COORDINATORE COMMISSIONE GIOVANI}

Ombretta Para, Firenze, Italy

\section{RESPONSABILE RAPPORTI ISTITUZIONALI}

Claudio Santini, Roma, Italy

\section{RESPONSABILE RAPPORTI CON LE REGIONI}

Alberto Fortini, Firenze, Italy

RESPONSABILE EVENTI E INIZIATIVE SPECIALI

Mauro Campanini, Novara, Italy

EDITOR IN CHIEF ITALIAN JOURNAL OF MEDICINE

Giorgio Vescovo, Padova, Italy
RESPONSABILE DEI

QUADERNI DELL'ITALIAN JOURNAL OF MEDICINE EDIZIONI ON LINE

Michele Meschi, Borgo Val di Taro (PR), Italy

\section{SUPERVISOR EDITOR DEI}

QUADERNI DELL'ITALIAN JOURNAL OF MEDICINE

Roberto Nardi, Bologna, Italy

RESPONSABILE SITO WEB E COMUNICAZIONE SOCIAL

Salvatore Lenti, Arezzo, Italy

Maurizia Gambacorta, Todi (PG), Italy

\section{WEB MANAGER E CONTENT EDITOR}

Giuseppe Oteri, Milano, Italy

Davide Ghilardi, Milano, Italy

RESPONSABILE SISTEMA GESTIONE QUALITÀ

Franco Berti, Roma, Italy

DPO

Alba Sciascera, Magenta (MI), Italy

\section{CONSULTA DEI PRESIDENTI}

Sandro Fontana, Biella, Italy

Salvatore Di Rosa, Palermo, Italy

Ido Iori, Reggio Emilia, Italy

Giovanni Mathieu, Pinerolo (TO), Italy

Antonino Mazzone, Legnano (MI), Italy

Carlo Nozzoli, Firenze, Italy

Mauro Campanini, Novara, Italy

Andrea Fontanella, Napoli, Italy 


\section{Italian Journal of Medicine}

\section{COORDINATORE}

David Terracina, Roma, Italy

\section{SEGRETARIO}

Andrea Montagnani, Grosseto, Italy

\section{DIRETTORI DEL DIPARTIMENTO \\ DELLA RICERCA CLINICA FADOI}

Filippo Pieralli, Firenze, Italy

Fulvio Pomero, Savigliano (CN), Italy

DIRETTORI DIPARTIMENTO

\section{PER LA FORMAZIONE E AGGIORNAMENTO}

Luigi Magnani, Voghera (PV), Italy

Roberta Re, Novara, Italy

\section{COORDINATORE SCIENTIFICO CENTRO STUDI FONDAZIONE FADOI}

Gualberto Gussoni, Milano, Italy

\section{DELEGATI SOCIETÀ SCIENTIFICHE COLLEGATE}

FISM Antonino Mazzone, Legnano (MI), Italy

SIF Luigi Magnani, Voghera (PV), Italy

Consulta Mauro Campanini, Novara (MI), Italy

cardiovascolare Michele Stornello, Siracusa, Italy

SIIA e ISO Michele Stornello, Siracusa, Italy Arcangelo Iannuzzi, Pomigliano d'Arco (NA), Italy

EFIM

Antonio Brucato, Milano, Italy

Lorenza Lenzi, Pomarolo (TN), Italy

Choosing wisely Roberto Frediani, Chieri (TO), Italy

\section{PROGETTI SPECIALI}

AGGIORN@FADOI

Giuliano Pinna, Marco Grandi

PROGETTO NUOVE TECNOLOGIE

Francesco Nasso, Flavio Tangianu

PROGETTO MEDICINA DI GENERE

Cecilia Politi

PROGETTO COMPETENCE

Flavio Tangianu

PROGETTO GOVERNANCE

Stefano De Carli, Andrea Montagnani, Fabrizio Colombo

PROGETTO HOSPITALIST

Francesco Orlandini

PROGETTO FINE VITA

Mauro Carbone, Fabio Gilioli

PROGETTO GASTROENTEROLOGIA \& FEGATO

Luca Fontanella, Paola Piccolo, Franco Radaelli, Giancarlo Parisi

PROGETTO NUTRIZIONE CLINICA

Roberto Risicato, Luciano Tramontano

PROGETTO MALATTIE INFETTIVE/ANTIBIOTICI

Claudio Santini, Massimo Giusti, Marco Falcone

PROGETTO ECOGRAFIA INTERNISTICA

Francesco Cipollini, Nicola Mumoli

PROGETTO MALATTIE RARE

Antonio Brucato, Antonella Paradiso

PROGETTO BPCO/NIV

Marco Candela, Giuseppe De Matthaeis, Francesco Ventrella

PROGETTO TROMBOSI

Mauro Silingardi, Matteo Giorgi Pierfranceschi, Pierpaolo Di Micco

PROGETTO SDO

Giovanni Mathieu

PROGETTO TRIAL

Giancarlo Agnelli, Antonio Ceriello, Leo Fabbri, Claudio Ferri,

Franco Radaelli, Paolo Verdecchia

\section{PRESIDENTE ANÍMO}

Gabriella Bordin, Castelfranco Veneto (TV), Italy 


\title{
QUADERNI - Italian Journal of Medicine
}

\author{
UN GIORNO IN MEDICINA. UN VIAGGIO ATTRAVERSO \\ LE OPINIONI DEGLI INTERNISTI FADOI \\ E DEGLI INFERMIERI ANİMO \\ Guest Editors: Ruggero Pastorelli, Gabriella Bordin
}

INTRODUZIONE

Un giorno in medicina. Un viaggio attraverso le opinioni degli internisti

FADOI .

A. Fontanella

\section{RASSEGNE}

Analisi della survey Un giorno in medicina. Un viaggio attraverso le opinioni degli internisti FADOI.

R. Pastorelli, C. Santini

Gli indicatori della qualità dell'assistenza nelle Unità Operative

di Medicina Interna secondo gli Internisti FADOI

S. Fiore, F. Tiratterra

L'attività clinica nelle Unità Operative di Medicina Interna:

gli aspetti gratificanti e quelli da modificare

D. Terracina, G. Campagna

Problematiche organizzative ed assistenziali nella gestione del paziente critico in Medicina Interna.

C. Nozzoli, F. Pieralli

I rapporti delle UO di Medicina Interna con il DEA e l'Ospedale ......

G. Ballardini, A. Franco

La Medicina Interna: ponte naturale fra Ospedale e Territorio. . . . . . . .

R. Re, T. Attardo, S. De Carli

Analisi della survey Un giorno in Medicina. Un viaggio attraverso le opinioni

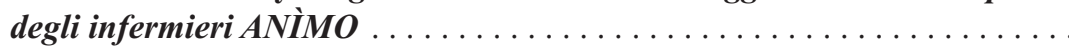

G. Bordin, R. Pastorelli

Un giorno in medicina: i risultati delle Survey FADOI e ANÌMO: quanto vicini, quanto lontani.

A. Maffettone, G. Bordin

Il core competence dell'infermiere in Medicina Interna: evoluzione e opportunità

F. Bertoncini, M. Piasentin

Gli esiti sensibili all'assistenza infermieristica in Medicina Interna .....

C. Gatta, L. Tesei 



\title{
Un giorno in medicina. Un viaggio attraverso le opinioni degli internisti FADOI
}

\author{
Andrea Fontanella \\ Presidente della Fondazione FADOI, Direttore del Dipartimento di Medicina e Pronto Soccorso, Ospedale Buon Consiglio \\ Fatebenefratelli, Napoli, Italia
}

Un giorno una farfalla guardando ammirata un millepiedi, osservava con stupore come riuscisse a muovere le sue zampette in quel modo straordinariamente coordinato. Lo chiamò e gli disse:

'Ehi Millepiedi, ma come fai a muovere tutti i tuoi piedi in quel modo?'. Il Millepiedi non ci aveva mai pensato, iniziò così a ragionarci sopra, e nel giro di pochi istanti perse la capacità di muoversi!

Questa sorta di parabola vuole cercare di riassumere il pensiero di Paul Watzlawick, psicologo e filosofo austriaco naturalizzato statunitense, eminente esponente della Scuola di Palo Alto, nonché seguace del costruttivismo. Egli coniò una mirabolante frase (che poi fu anche il titolo di un suo famoso libro): 'Guardarsi dentro rende ciechi'.

Con questa locuzione si voleva intendere che cercare di interpretare i propri moti interiori (pensieri, emozioni, sensazioni e comportamenti) dando loro delle spiegazioni di tipo causa-effetto, rischiava di farci impigliare maggiormente nei problemi che cercavamo di risolvere.

La FADOI, però, ha avuto il coraggio di fare questo, guardando a una filosofia ben più antica, all'epoca ellenica, quando gli uomini non avevano paura di guardarsi dentro. Oggi molte persone sono fobiche degli stati interiori. Realizzano quello che si è chiamato evitamento esperienziale. Invece, sulla scorta dell'insegnamento antico il processo di crescita personale, oltre che di team, deve passare soprattutto at-

Corrispondente: Andrea Fontanella, Presidente della Fondazione FADOI, Direttore del Dipartimento di Medicina e Pronto Soccorso, Ospedale Buon Consiglio Fatebenefratelli, via Manzoni 220, 80124 Napoli, Italia.

E-mail: andreafontanella52@gmail.com

Articolo pubblicato secondo la Creative Commons Attribution NonCommercial 4.0 License (CC BY-NC 4.0).

${ }^{\circ}$ Copyright: the Author(s), 2020

Licensee PAGEPress, Italy

QUADERNI - Italian Journal of Medicine 2020; 8(3):1-2 traverso un processo di graduale scoperta di sé stessi, il famoso conosci te stesso. Nosce te ipsum, frase latina che traduce il greco $\gamma \nu \tilde{\omega} \vartheta \iota$ $\sigma \varepsilon \alpha v \tau o ́ v$, uno degli apoftegmi attribuiti ai Sette Sapienti, che, inciso sul frontone del tempio di Apollo in Delfi, esortava gli uomini al riconoscimento della propria condizione e limitatezza umana. Socrate ne fece la sua massima preferita, interpretandola come un invito a considerare i limiti della conoscenza umana prima di procedere nella via del sapere e quindi della virtù.

$\mathrm{Se}$, però, ci si vuole guardare dentro senza diventare ciechi, si devono trovare degli occhiali che permettano di evitare l'errore epistemologico della linearità del pensiero... e questi occhiali sono rappresentati dalla presenza.

Per presenza si intende quello stato di attenzione naturale non giudicante che si ha quando si è completamente dentro una certa situazione.

La FADOI, quindi, è andata alla scoperta di sé stessa, cercando di comprendere le opinioni e gli orientamenti degli Internisti espressi al di fuori dell'atmosfera congressuale ma nel contesto del loro operare quotidiano, nell'ambito delle singole realtà. Avere consapevolezza di sé, infatti, è la base per potersi migliorare ed evolvere e rappresenta per la FADOI il modo migliore per orientare le iniziative societarie nelle attività formative, ma anche nel confronto con le istituzioni. Altri scopi sono poi stati anche quelli di fornire una rappresentazione nazionale degli orientamenti degli Internisti FADOI sui temi oggetto della survey, di cogliere le differenze regionali e macroregionali, e di stimare se, da parte degli Internisti esista una sensibilità reale e non puramente accademica verso gli aspetti organizzativi ed evolutivi della MI.

La realtà sanitaria in Italia è particolarmente complessa, la Medicina Interna è la specialità più rappresentata sul territorio nazionale. Ogni ospedale, da quelli metropolitani di maggiori dimensioni e di elevata specializzazione a quelli periferici con minore dotazione di posti letto e di specialità, presenta nel proprio interno una o più strutture complesse di medicina. Si stima che in Italia gli internisti siano circa 
11.000. Nonostante questo dato numerico rilevante e che non ha eguali nelle altre specialità mediche e chirurgiche, spesso risulta difficile sia alla popolazione sia agli organi decisori nazionali e regionali identificare le nostre competenze; siamo, in poche parole, difficilmente identificabili in uno specifico contesto. Le UO di MI operano in strutture ospedaliere anche molto diverse fra loro e in contesti territoriali differenti. I medici delle UO di MI sono eterogenei per età anagrafica, formazione e specializzazione più di quanto lo siano $\mathrm{i}$ Colleghi delle specialità d'organo. Va, inoltre, considerato che, date le attuali difficoltà di molti sistemi sanitari regionali, in alcune realtà si può essere sviluppato nel tempo il gradimento verso un'attività prestazionale e/o turnistica (es. turni preferibilmente notturni - nocturnalist o settimanali - 7 on/7 off) e su pazienti preferibilmente non critici e ambulatoriali.

Nonostante questa incredibile eterogeneità, dettata dalla stessa multiparametrica complessità, le risposte raccolte dalla survey sono state fortemente rassicuranti. I temi principali attorno ai quali si è svolta l'indagine sono stati: l'identificazione di indicatori più idonei a valutare la qualità della cura in una UO di MI, quali fossero gli aspetti più soddisfacenti e più importanti dell'attività di reparto, nonché gli aspetti peculiari e quelli da modificare, quale fosse la propensione alla cura dei pazienti critici e, infine, come fossero i rapporti tra le UO di MI, tra l'Ospedale e il Dipartimento Emergenza e Accettazione (DEA). Se i risultati della survey sono stati mirabilmente presentati, sviluppati e commentati nel principale articolo della monografia, da parte del titolare intellettuale nonché ideatore della survey, dott. Ruggero Pastorelli, coadiuvato da Claudio Santini, i singoli argomenti della stessa sono stati magistralmente esplicitati dai differenti autori di questa monografia, compreso l'importante contributo fornito dagli infermieri dell'ANÌMO.

Orbene a tali domande si è osservata una rassicurante concordanza di opinioni nel popolo degli Internisti ospedalieri italiani, cosa assolutamente non scontata, vista la non omogeneità formativa e organizzativa degli Internisti, che, spesso, differiscono per la diversità della formazione post-laurea, dei percorsi professionali, del tipo di ospedale e della sua localizzazione geografica.

Come avete avuto modo di leggere nel testo ci si trova di fronte ad una specialità più omogenea del previsto nonostante le rimarcate diversità.

Il dato veramente rassicurante che viene fuori da questa survey è che gli Internisti confermano l'importanza della cura della persona e della complessità clinica con metodo olistico, ma individuano anche come priorità la continuità assistenziale, la comunicazione, la cultura medica del fine vita e la collaborazione con gli Infermieri della UO e con gli altri Specialisti.

Le vere dissonanze si trovano nei rapporti con le Direzioni Strategiche e il DEA, si evidenzia, infatti, una significativa distanza fra gli atti delle Direzioni Sanitarie e i Medici Internisti nella percezione dell'importanza strategica della Medicina interna e della sua attività complessiva negli ospedali italiani. È, inoltre, rimarcato un insufficiente coordinamento fra le attività di MI e l'attività di ricovero di DEA e PS.

Questa survey, in definitiva, è stata di rilevante importanza non solo per avere dato una realistica fotografia della Medicina Interna italiana vista dall'interno (una sorta di selfie), ma, soprattutto, per l'importante contributo che fornisce alla FADOI per le sue scelte strategiche, sia in senso formativo sia nei rapporti con le Istituzioni, locali e nazionali.

Essa rimarca sostanzialmente come il fermo volere che accomuna tutto il popolo degli Internisti sia principalmente quello di non ostinarsi a modificare la struttura, ma il modo di lavorare e di pensare (programmazione, coordinamento, trasparenza, accountability), focalizzarsi sui problemi e sui sistemi in grado di risolverli, mettere la persona al centro, gestire la conoscenza e il talento con la stessa attenzione con cui si gestiscono i soldi, cosa che è poi il vero spirito della Medicina Interna. 


\title{
Analisi della survey Un giorno in Medicina. Un viaggio attraverso le opinioni degli internisti FADOI
}

\author{
Ruggero Pastorelli, ${ }^{1}$ Claudio Santini ${ }^{2}$ \\ ${ }^{1}$ Medicina Interna, Colleferro (Roma); ${ }^{2}$ UOC Medicina Interna, Ospedale Vannini, Roma, Italia
}

\section{RIASSUNTO}

Gli Internisti italiani aderenti alla FADOI (Federazione Associazioni Dirigenti Ospedalieri Internisti) sono stati coinvolti in una survey web-based indirizzata a raccogliere gli orientamenti di coloro che vivono la realtà clinica ospedaliera su: i) gli indicatori più idonei a valutare la qualità della cura in una Unità Operativa di Medicina Interna; ii) gli aspetti più soddisfacenti e più importanti dell'attività di reparto, nonché gli aspetti peculiari e quelli da modificare; iii) la propensione alla cura dei pazienti critici; ed infine iv) gli attuali rapporti tra le Unità Operative di Medicina Interna, l'Ospedale e il Dipartimento Emergenza e Accettazione. Alla survey hanno aderito 740 Internisti rappresentativi della realtà della Medicina Interna italiana. L'elaborazione dei dati, condotta dal Centro Studi FADOI, ha evidenziato: i) una specialità più omogenea dell'atteso; ii) la propensione ad indicatori di qualità correlati all'organizzazione dell'attività clinica piuttosto che di carattere amministrativo; iii) conferma del valore nella pratica clinica delle caratteristiche fondamentali della Medicina Interna; iv) propensione all'autonomia nelle procedure, esami ecografici e ventilazione non invasiva (NIV); v) propensione alla cura del paziente critico; vi) opportunità di rivedere e aggiornare le modalità di svolgimento della visita collegiale; vii) criticità fondamentalmente rappresentate dai rapporti con l'Ospedale e il Dipartimento Emergenza e Accettazione.

\section{Introduzione}

La FADOI è sempre stata caratterizzata da un alto livello di attenzione verso l'evoluzione della Medicina Interna (MI). Essa ha considerato e anche delineato le possibili caratteristiche dell'Internista del III millennio, ${ }^{1}$ la sua evoluzione centrata sulla formazione continua e l'adeguamento tecnologico, la declinazione della sua attività in nuovi modelli organizzativi (intensità di cure, hospitalist, ...).

Tuttavia, la possibile contestualizzazione di nuovi scenari organizzativi nelle Unità Operative (UO) di MI, impone almeno due riflessioni: i) le UO di MI operano in strutture ospedaliere anche molto diverse fra loro e in contesti territoriali differenti; ii) i medici delle UO di MI sono eterogenei per età anagrafica, formazione e specializzazione più di quanto lo siano i Colleghi delle specialità d'organo (Cardiologia, Nefrologia, ...) e ciò appare confermato, oltre che da quotidiane osservazioni nella vita reale,

Corrispondente: Ruggero Pastorelli, Medicina Interna, via Fontana dell'Oste 13/E, 00034 Colleferro (RM), Italia. E-mail: rugpas@inwind.it

Articolo pubblicato secondo la Creative Commons Attribution NonCommercial 4.0 License (CC BY-NC 4.0).

${ }^{\circ}$ Copyright: the Author(s), 2020

Licensee PAGEPress, Italy

QUADERNI - Italian Journal of Medicine 2020; 8(3):3-13 anche da alcuni report della letteratura internazionale. $^{2-4}$

Inoltre, va considerato che, date le attuali difficoltà di molti sistemi sanitari regionali, in alcune realtà si può essere sviluppato nel tempo il gradimento verso una attività prestazionale e/o turnistica (es., turni preferibilmente notturni-nocturnalist o settimanali -7 on/7 off) e su pazienti preferibilmente non critici e ambulatoriali.

Pertanto, dopo uno studio pilota condotto nel Lazio, è nata l'iniziativa di coinvolgere gli Internisti italiani aderenti alla FADOI in una survey denominata Un giorno in Medicina. Un viaggio attraverso le opinioni degli Internisti FADOI.

Tale survey consiste di una rilevazione tramite 3 questionari: i) di una serie di opinioni e di orientamenti degli Internisti espressi al di fuori dell'atmosfera congressuale; ii) per alcuni versi a completamento dei messaggi e considerazioni top down della letteratura, ${ }^{5-7}$ iii) potenzialmente utili a orientare le iniziative societarie nelle attività formative, ma anche nel confronto con le istituzioni.

Gli argomenti oggetto dell'indagine sono stati: i) gli indicatori più idonei a valutare la qualità della cura in una UO di MI; ii) gli aspetti più soddisfacenti e più importanti dell'attività di reparto, nonché gli aspetti peculiari e quelli da modificare; iii) la propensione alla cura dei pazienti critici; iv) ed infine gli attuali rapporti tra le UO di MI, l'Ospedale e il Dipartimento Emergenza e Accettazione (DEA).

I dati potrebbero: i) fornire una rappresentazione nazionale degli orientamenti degli Internisti FADOI sui temi oggetto della survey; ii) cogliere le differenze 
regionali e macroregionali; iii) indicare se la sensibilità degli Internisti agli aspetti organizzativi ed evolutivi della MI è diffusa, reale o prevalentemente accademica; iv) essere utili per orientare FADOI nelle iniziative societarie e nel confronto con le istituzioni.

\section{Materiali e Metodi}

La survey, condotta tramite il Centro Studi della FADOI, è stata proposta agli Internisti FADOI nel periodo marzo-aprile 2019 in modalità web-based.

Essa comprendeva tre questionari $(\mathrm{A}, \mathrm{B}, \mathrm{C})$ volti a individuare: i) gli indicatori che gli Internisti ritengono più idonei per la valutazione della qualità delle cure in una UO di MI (30 item) nel questionario A; ii) gli aspetti più soddisfacenti $(8 \mathrm{item})$ e più importanti $(12$ item) dell'attività di reparto, gli aspetti peculiari $(8$ item), quelli da modificare (11 item) e alcuni aspetti della cura dei pazienti critici (4 item) nel questionario $\mathrm{B}$; iii) le opinioni degli Internisti sui rapporti tra le UO di MI con l'Ospedale e il DEA (Dipartimento Emergenza Accettazione) (6 e 4 item rispettivamente) nel questionario $\mathrm{C}$.

Ad ogni item riportato nei questionari è stato chiesto di attribuire un valore tra 1 e $10(1=$ irrilevante ; $10=$ estremamente importante).

Inoltre, a ciascun partecipante è stato chiesto di indicare età, disciplina di specializzazione, eventuale incarico di direzione della UO, caratteristiche del proprio ospedale quali localizzazione regionale, numero posti letto, presenza di DEA o di Pronto Soccorso (PS).

Gli item da inserire nei questionari A,B,C sono stati individuati da un panel di Esperti dell'Area Organizzativa della FADOI Lazio. Al fine di raggiungere un consenso in maniera efficiente e rapida focalizzando l'attenzione sugli aspetti rilevanti degli obiettivi, è stata utilizzata una versione semplificata della metodica Delphi ${ }^{8,9}$ A ciascuno degli esperti è stato chiesto di elencare gli argomenti che a suo giudizio meritavano di essere inseriti nei tre questionari. Successivamente, è stata avviata una discussione collegiale fino alla definizione di una lista ristretta di item. Le voci della lista ristretta sono state votate separatamente da ciascun Esperto secondo i parametri di rilevanza assoluta e fattibilità utilizzando una scala da 1 a 10 . Successivamente le voci a punteggio più alto sono state inserite nei questionari.

È stato quindi effettuato uno studio pilota proponendo i questionari agli iscritti alla FADOI Lazio (novembre 2018, 68 risposte rappresentative della distribuzione geografica delle UO di MI del Lazio). Verificata la fattibilità, la survey è stata proposta a tutti gli Internisti italiani iscritti a FADOI (marzo-aprile 2019).

I dati finali sono stati elaborati in forma anonima dal Centro Studi FADOI.

\section{Risultati}

I dati generali degli Internisti che hanno partecipato alla survey sono riassunti nella Figura 1 e dimostrano che il campione è rappresentativo della distribuzione geografica e delle caratteristiche generali delle Medicine Interne italiane. L'elevato numero di risposte dimostra che la sensibilità degli Internisti agli aspetti organizzativi ed evolutivi della MI è diffusa e reale.

Le risposte ai questionari $\mathrm{A}, \mathrm{B}$ e $\mathrm{C}$ sono state rispettivamente 740, 662 e 647 . Complessivamente le risposte complete sono state 647 e quelle parziali 93.

La provenienza geografica delle risposte (Nord $n$. 247, Centro-Nord n. 170, Centro n. 163, Sud e Isole n. 160) corrisponde alla numerosità regionale degli Internisti iscritti a FADOI al 31.12.2018 per cui il campione si può considerare globalmente rappresentativo degli Internisti FADOI italiani [Nota: Nord: Piemonte, Liguria, Lombardia, Veneto, Trentino Alto Adige, Friuli Venezia Giulia; Centro Nord: Toscana, Emilia Romagna; Centro: Lazio, Marche, Umbria, Abruzzo, Molise; Sud e Isole: Campania, Basilicata, Puglia, Calabria, Sicilia, Sardegna].

Le fasce di età più rappresentate nelle risposte sono state $30-45$ anni $(33,9 \%)$ e $\geq 56$ anni $(32 \%)$.

La disciplina di specializzazione degli Internisti coinvolti (per il 23,5\% Responsabili di UO) è risultata essere la Medicina Interna nel 59,5\% dei casi.

Quanto alle caratteristiche degli ospedali sede dell'attività degli Internisti, risultano equamente rappresentati sia gli ospedali con differente numero di posti letto $(<200,200-400,>400)$ sia quelli sede di DEA I e II livello o di PS.

\section{Questionario A (n. 740 risposte)}

I punteggi assegnati agli item sono riassunti nella Tabella 1 . Vi è stata una concentrazione delle risposte nelle fasce di voto 7 e 8 con rare escursioni nella fascia 6 .

Il punteggio medio assegnato agli indicatori correlati ai processi e a risorse/organizzazione (rispettivamente 8,28 e 8,12 ) è risultato maggiore a quello riportato dagli indicatori correlati agli esiti $(7,37)$ e a quelli amministrativi $(7,22)$. Nella Tabella 2 sono riportati gli indicatori che hanno riportato il punteggio più alto $(>8)$.

\section{Questionario B (n. 662 risposte)}

I punteggi assegnati agli aspetti dell'attività di reparto più soddisfacenti, più importanti, peculiari e da modificare sono riassunti nella Tabella 3 e nelle Figure $2,3,4$ e 5 . Anche in questo caso vi è stata una sostanziale concentrazione delle risposte nelle fasce di voto 7 e 8 con rare escursioni nella fascia 6 . Il punteggio medio relativo agli aspetti più soddisfacenti $(8,07)$ è risultato inferiore a quelli relativi agli aspetti peculiari $(8,38)$, da modificare $(8,32)$ e più importanti $(8,27)$. 


\section{Cura del paziente critico}

Le risposte alle domande relative alla cura dei pazienti critici sono riportate nella Tabella 4.

Se ne ricava che solo il $41 \%$ degli delle UO di MI dispone già di posti letto monitorizzati per la cura del paziente critico. Il dato non presenta significative differenze macroregionali. Il 93\% degli Internisti ritiene che la disponibilità di posti letto monitorizzati possa essere un vantaggio per l'attività e che sarebbe pronto ad aggiornarsi o a formarsi sull'argomento.

\section{Questionario C (n. 647 risposte)}

\section{Rapporti con l'ospedale}

Le risposte sono riportate nelle Tabelle 5 e 6 .

Benché il 57\% degli Internisti ritenga che la pro-

\begin{tabular}{|c|c|c|c|c|}
\hline \multicolumn{2}{|c|}{ RISPOSTE: } & \multicolumn{3}{|c|}{ Età dei rispondenti } \\
\hline Complete & 647 & $<30$ & 39 & $5,8 \%$ \\
\hline Parziali & 93 & $46-55$ & 169 & $22,8 \%$ \\
\hline Tot & 740 & 256 & 237 & $32 \%$ \\
\hline Risposte per macroaree regionali \\
\hline Nord & $247(33,4 \%)$ & 211 & 36 & 931 \\
\hline Centro-Nord & $170(23 \%)$ & 153 & 17 & 639 \\
\hline Centro & $163(22 \%)$ & 141 & 22 & 551 \\
\hline Sud e Isole & $160(21,6 \%)$ & 142 & 18 & 570 \\
\hline
\end{tabular}

\begin{tabular}{|c|c|c|}
\hline \multicolumn{2}{|c|}{ Posti letto Ospedale } \\
\hline$<200$ & 234 & $31,6 \%$ \\
\hline $200-400$ & 245 & $33,1 \%$ \\
\hline$>400$ & 261 & $35,3 \%$ \\
\hline
\end{tabular}

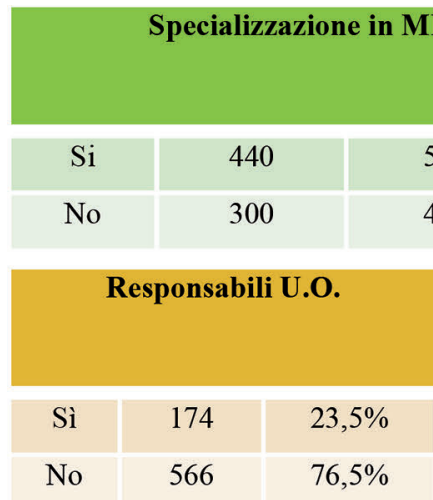

\section{MI}

$40,5 \%$

\section{Distribuzione geografica Ospedali}

\begin{tabular}{|c|c|c|c|}
\hline & $<200$ & $200-400$ & $>400$ \\
\hline Nord & 61 & 86 & 100 \\
\hline Centro-Nord & 63 & 41 & 66 \\
\hline Centro & 50 & 65 & 48 \\
\hline Sud e Isole & 60 & 53 & 47 \\
\hline
\end{tabular}
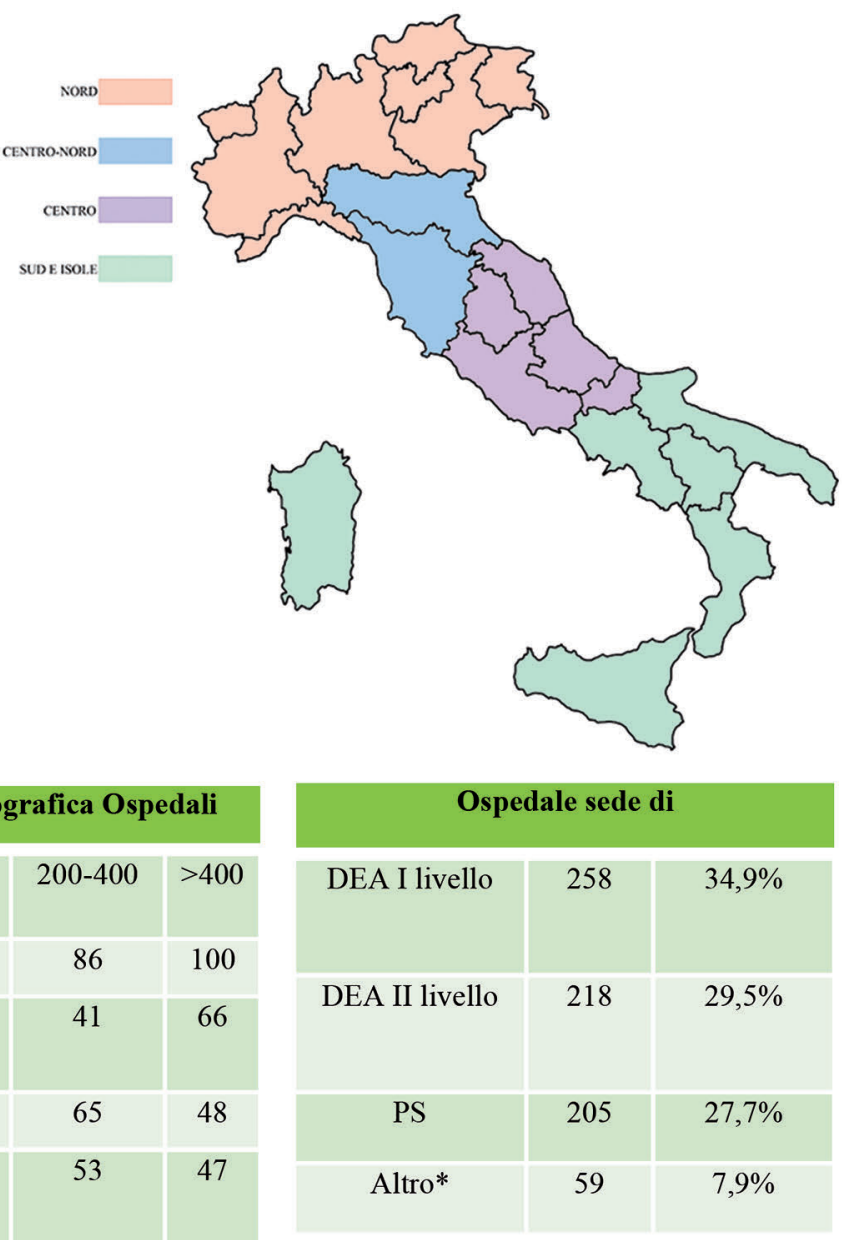

59,5\% $\quad$ Geriatria

Endocrinologia

Cardiologia

Gastroenterologia

Ematologia, pneumologia...

Figura 1. Dati generali Internisti partecipanti alla survey. 
pria UO fornisca una visione forte della MI e percepisca il proprio ruolo come importante $(75 \%)$ e non ancillare (25\%), il 71\% ritiene che gli atti della Direzione Strategica non tengono in giusto conto l'importanza dell'attività delle UO di MI.

\section{Rapporti con il DEA}

La grande maggioranza degli Internisti (Tabelle 6 e 7) ritiene che le consulenze internistiche nel
DEA o PS sono importanti per il percorso del paziente $(82 \%)$ e per definire il flusso dei ricoveri $(94 \%)$; tuttavia, mai $(51 \%)$ o raramente $(32 \%)$ viene richiesta la consulenza internistica prima di un ricovero in una UO di MI. Inoltre, tenendo conto di aree di degenza anche nel DEA, i ricoveri in MI sarebbero sostanzialmente appropriati nel $63 \%$ dei casi, in minima parte appropriati nel $34 \%$, non appropriati nel $3 \%$.

Tabella 1. Questionario A. Indicatori di qualità della cura (n. 740 risposte).

Indicatori amministrativi correlati all'attività

Numero di re-ricoveri entro un mese $\quad 7,85$

\begin{tabular}{ll}
\hline Percentuale ricoveri dal Pronto Soccorso & 7,50
\end{tabular}

\begin{tabular}{ll}
\hline Percentuale di dimissioni a domicilio & 7,25
\end{tabular}

Numero di pazienti appoggiati in altro reparto 7,24

Numero di pazienti in letti aggiunti in reparto 7,18

Numero dimissioni l'anno 7,07

\begin{tabular}{ll}
\hline Peso DRG generati & 7,05 \\
\hline
\end{tabular}

\begin{tabular}{ll} 
Degenza media & 6,99 \\
\hline
\end{tabular}

Punteggio medio $\quad 7,22$

Indicatori correlati a risorse e organizzazione

Rapporto n. infermieri/n. pazienti 8,98

Presenza in visita di uno stesso Medico per almeno 5 giorni/settimana 8,58

Possibilità di controlli ambulatoriali post-dimissione 8,28

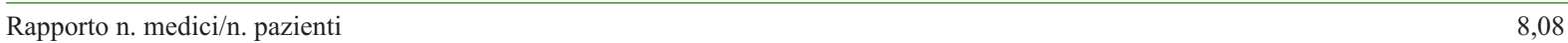

\begin{tabular}{ll}
\hline Utilizzo di lettera di dimissione strutturata & 8,08
\end{tabular}

Presenza del Direttore UO nelle attività cliniche 8,06

Individuazione del Medico che prende in carico il paziente 7,86

$\begin{array}{ll}\text { Percentuale ore di servizio svolte dai Medici in attività di guardia } & 7,64\end{array}$

Presenza dello stesso infermiere nella visita quotidiana 7,58

Punteggio medio $\quad 8,12$

Indicatori correlati ai processi

\begin{tabular}{ll}
\hline Gestione del fine vita & 8,50
\end{tabular}

Meeting clinici e discussioni collegiali 8,45

Possibilità di effettuare direttamente esami strumentali ecografici e procedure 8,39

Percorsi diagnostico-terapeutici strutturati e condivisi 8,35

\begin{tabular}{ll}
\hline Possibilità di effettuare NIV & 8,25
\end{tabular}

Organizzazione per continuità assistenziale 8,02

Gestione rischio clinico 8,00

$\begin{array}{rrr} & \text { Punteggio medio } & \mathbf{8 , 2 8}\end{array}$

Indicatori correlati agli esiti

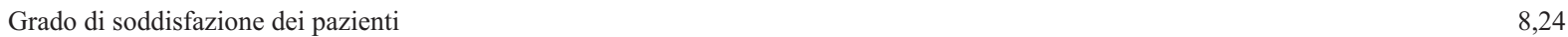

\begin{tabular}{ll}
\hline Frequenza insorgenza delle lesioni da pressione & 8,04
\end{tabular}

\begin{tabular}{ll}
\hline Numero delle cadute dei pazienti & 7,40 \\
\hline
\end{tabular}

\begin{tabular}{ll}
\hline Numero dei contenziosi & 7,01 \\
\hline
\end{tabular}

Percentuale di mortalità 6,87

Percentuale di pazienti sottoposti a cateterismo vescicale 6,71

\begin{tabular}{lrl} 
& & 6,71 \\
\hline Percentuale di pazienti sottoposti a cateterismo vescicale & Punteggio medio & $\mathbf{7 , 3 7}$
\end{tabular}

DRG, diagnosis-related group; NIV, ventilazione non invasiva; UO, Unità Operativa. 


\section{Discussione}

\section{Indicatori della qualità della cura in Medicina Interna}

Benché il management delle strutture ospedaliere continui ad essere ispirato prevalentemente da criteri di produttività piuttosto che da criteri di clinical governance e dagli esiti delle cure, l'orientamento degli Internisti è nettamente a favore degli indicatori associati alle risorse e la loro organizzazione e ai processi (Tabella 1) piuttosto che verso gli indicatori di carattere prevalentemente amministrativo di solito proposti dalle Direzioni e dai decisori istituzionali delle politiche sanitarie. Questi, infatti, hanno riportato nella survey il punteggio medio più basso $(7,22)$. Nella Tabella 2 sono riportati gli indicatori che hanno riportato il punteggio più alto $(>8)$.

In effetti, in questo ambito, medici e gestori sembrano avere una visione leggermente diversa della sanità poiché i primi sono orientati più all'azione che alla pianificazione, si identificano maggiormente con la propria professione, la cura e l'organizzazione dell' attività clinica e ritengono fondamentale la propria autonomia in tali ambiti. ${ }^{10}$ A questo riguardo sono state registrate differenze anche fra gli orientamenti dei Direttori di UO e gli altri medici della UO (Tabella 8) che meriterebbero forse un ulteriore futuro approfondimento.

Inoltre, va considerato che la letteratura ${ }^{11-16}$ riporta che molti indicatori proposti dai gestori della sanità non appaiono i più idonei a definire la qualità della cura di una UO di MI poiché spesso sono influenzati da criticità di sistema (difficile transizione ospedaleterritorio, difficoltà della medicina territoriale, insufficiente clinical governance, criteri di ricovero da PS e DEA non condivisi, ecc.) piuttosto che dalla performance della una singola UO.

\section{Gli aspetti dell'attività più soddisfacenti, più importanti, peculiari e da modificare}

Nella survey vengono confermati come elementi peculiari dell'attività internistica la visione globale, il metodo, la valutazione delle priorità diagnostiche e terapeutiche, il management delle comorbilità e le diagnosi difficili (Tabella 3; Figura 4). Gli aspetti più soddisfacenti riguardano i buoni rapporti con i Colleghi di reparto, la comunicazione medico-paziente e la risoluzione della patologia del paziente (Figura 2). Gli aspetti più importanti e i relativi punteggi assegnati sono riportati nella Figura 3. I principali fattori che caratterizzano negativamente l'attività dipendono per larga parte da cause non direttamente collegate alla UO: dotazione organica, tecnologia non adeguata, organizzazione del lavoro e carico assistenziale, anche al di fuori del reparto (Figura 5).

Appare opportuno sottolineare che, benché la collaborazione con i Colleghi di reparto e gli Infermieri sia considerata tra gli aspetti più importanti dell'attività, la visita collegiale è associata al punteggio più basso tanto nelle attività più soddisfacenti che in quelle più importanti e peculiari (Tabella 3; Figure $2,3,4)$. Il dato, certamente non atteso, non si presta ad una interpretazione univoca. La visita collegiale è certamente un aspetto fondamentale dell'attività dell'Internista, ${ }^{17-20}$ ma probabilmente le modalità del suo svolgimento meritano di essere riviste prevedendo un maggiore coinvolgimento del team e azioni mirate alla soluzione coordinata di problemi clinici e gestionali e ad un arricchimento della prestazione complessiva.

Altro aspetto da sottolineare è che la comunicazione medico-paziente e la possibilità di svolgere direttamente indagini strumentali, procedure e NIV sono associate ad un punteggio elevato sia tra le attività di reparto più soddisfacenti che tra quelle più importanti.

Tabella 2. Indicatori di qualità a punteggio più alto (n. 740 risposte).

\begin{tabular}{|c|c|}
\hline Rapporto n. infermieri/n. pazienti & 8,98 \\
\hline Presenza in visita di uno stesso Medico per almeno 5 giorni/settimana & 8,58 \\
\hline Gestione del fine vita & 8,50 \\
\hline Meeting clinici e discussioni collegiali & 8,45 \\
\hline Possibilità di effettuare direttamente esami strumentali ecografici e procedure & 8,39 \\
\hline Percorsi diagnostico-terapeutici strutturati e condivisi & 8,35 \\
\hline Possibilità di controlli ambulatoriali post-dimissione & 8,28 \\
\hline Possibilità di effettuare NIV & 8,25 \\
\hline Grado di soddisfazione dei pazienti & 8,24 \\
\hline Rapporto n. medici/n. pazienti & 8,08 \\
\hline Presenza del Direttore UO nelle attività cliniche & 8,06 \\
\hline Organizzazione per continuità assistenziale & 8,02 \\
\hline Gestione rischio clinico & 8,00 \\
\hline
\end{tabular}

NIV, ventilazione non invasiva; UO, Unità Operativa. 
Tabella 3. Questionario B. Gli aspetti dell'attività (n. 662 risposte).

Aspetti più soddisfacenti

\begin{tabular}{ll}
\hline Rapporto Medico/Paziente 8,87 \\
\hline
\end{tabular}

\begin{tabular}{ll}
\hline Rapporti/confronto con Colleghi & 8,39 \\
\hline
\end{tabular}

\begin{tabular}{ll}
\hline Risoluzione patologia paziente & 8,34
\end{tabular}

Possibilità di svolgere direttamente indagini strumentali e procedure 8,12

Possibilità di svolgere NIV (ventilazione non invasiva) 8,06

\begin{tabular}{ll}
\hline Meeting di reparto & 7,77 \\
\hline
\end{tabular}

\begin{tabular}{ll}
\hline Management clinico & 7,74
\end{tabular}

Visita collegiale 7,31

\begin{tabular}{rrr}
\hline 8,07 \\
\hline
\end{tabular}

Aspetti più importanti

\begin{tabular}{ll}
\hline Collaborazione Infermieri & 8,89
\end{tabular}

\begin{tabular}{ll}
\hline Collaborazione Colleghi Reparto & 8,71
\end{tabular}

\begin{tabular}{ll}
\hline Metodo internistico & 8,69 \\
\hline
\end{tabular}

\begin{tabular}{ll}
\hline Tempo a disposizione per l'assistenza & 8,65 \\
\hline
\end{tabular}

\begin{tabular}{ll}
\hline Collaborazione altri Specialisti & 8,59 \\
\hline
\end{tabular}

\begin{tabular}{ll}
\hline Relazioni con Pazienti/Familiari & 8,55 \\
\hline
\end{tabular}

$\overline{\text { Continuità assistenziale }} 8,42$

\begin{tabular}{ll}
\hline Attività strumentali & 8,26
\end{tabular}

Percorsi predefiniti per il trasferimento pazienti 7,97

\begin{tabular}{ll}
\hline Possibilità di svolgere NIV (ventilazione non invasiva) & 7,97 \\
\hline
\end{tabular}

\begin{tabular}{ll}
\hline Meeting post-visita & 7,64
\end{tabular}

\begin{tabular}{ll}
\hline Visita collegiale & 6,90 \\
\hline
\end{tabular}

\begin{tabular}{rrr}
\hline & Punteggio medio & 8,27
\end{tabular}

Aspetti peculiari

\begin{tabular}{ll}
\hline Visione globale & 8,87 \\
\hline Cominicazione PazientiFamiliai
\end{tabular}

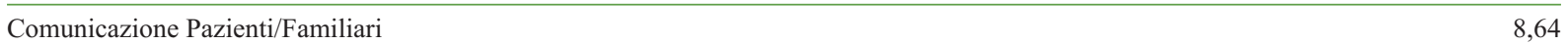

\begin{tabular}{ll}
\hline Valutazione priorità terapeutiche & 8,52 \\
\hline
\end{tabular}

\begin{tabular}{ll}
\hline Metodo interni stico & 8,48 \\
\hline
\end{tabular}

\begin{tabular}{ll}
\hline Valutazione priorità diagnostiche & 8,46
\end{tabular}

\begin{tabular}{ll}
\hline Management comorbilità & 8,33 \\
\hline
\end{tabular}

\begin{tabular}{ll}
\hline Diagnosi difficili 8,24 \\
\hline Visia collegiale
\end{tabular}

\begin{tabular}{ll}
\hline Visita collegiale & 7,53 \\
\hline
\end{tabular}

$\begin{array}{rrr}8,38 & \text { Punteggio medio } & 8\end{array}$

Aspetti da modificare

\begin{tabular}{ll}
\hline Dotazione organica & 8,68
\end{tabular}

\begin{tabular}{ll}
\hline Organizzazione lavoro e turni & 8,68 \\
\hline
\end{tabular}

\begin{tabular}{ll}
\hline Eccesso di pazienti in carico & 8,67 \\
\hline
\end{tabular}

\begin{tabular}{ll}
\hline Tempo per aggiornamento & 8,65
\end{tabular}

\begin{tabular}{ll}
\hline Pazienti in altri reparti (appoggi) & 8,39
\end{tabular}

\begin{tabular}{ll}
\hline Tecnologia non adeguata & 8,26 \\
\hline Comuniazione Pazienti/2amiliri
\end{tabular}

\begin{tabular}{ll}
\hline Comunicazione Pazienti/Familiari 8,25 \\
\hline Collatazion ColeohiRearto
\end{tabular}

Collaborazione Colleghi Reparto 8,15

$\overline{\text { Collaborazione/confronto con altri Specialisti } 8,03}$

\begin{tabular}{ll}
\hline Burocrazia & 7,96
\end{tabular}

\begin{tabular}{ll}
\hline Discontinuità assistenziale & 7,81 \\
\hline
\end{tabular}

Punteggio medio $\quad \mathbf{8 , 3 2}$ 


\section{Cura del paziente critico}

Solo una minoranza delle UO di MI, senza significative differenze macroregionali, dispone già di posti letto monitorizzati per la cura del paziente critico (Tabella 4). Su questo argomento il $93 \%$ degli Internisti si è dichiarato favorevole alla formazione e all'aggior-

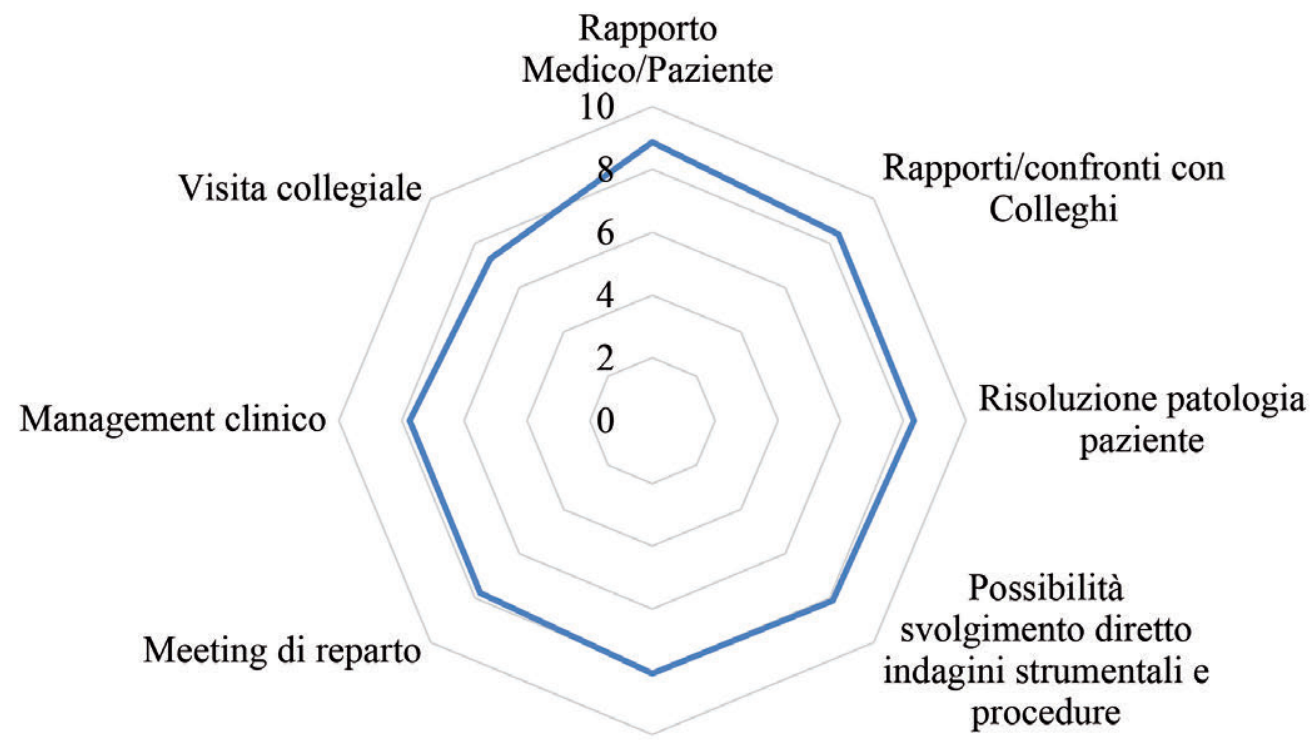

Possibilità di svolgere

NIV

Figura 2. Gli aspetti più soddisfacenti dell'attività. Concentrazione delle risposte nelle fasce di voto 7 e 8 (punteggio medio 8,07); il punteggio più alto è stato assegnato all'item Rapporto Medico/Paziente $(8,87)$, il punteggio più basso all'item Visita collegiale (7,31). NIV, ventilazione non invasiva.

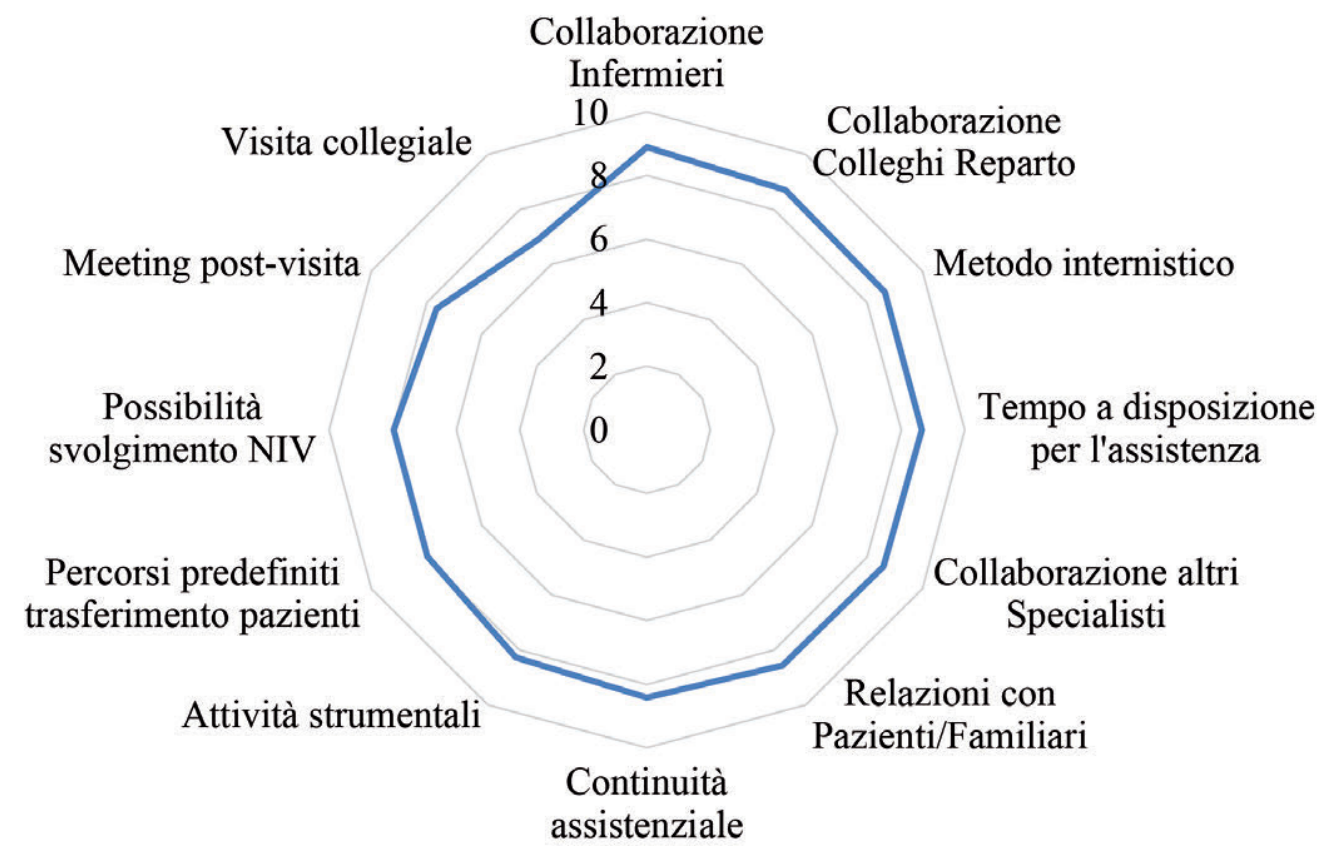

Figura 3. Gli aspetti più importanti dell'attività. Concentrazione degli item prevalentemente nella fascia di voto 8 , con qualche escursione nella fascia del 7 e una escursione nella fascia del 6 (punteggio medio 8,27). Il punteggio più alto è stato assegnato all'item Collaborazione Infermieri $(8,89)$, il punteggio più basso all'item Visita collegiale $(6,90)$. NIV, ventilazione non invasiva. 
namento. Questo tipo di risposta è in linea con gli obiettivi della moderna Medicina Interna.

Infatti, negli ospedali italiani afferisce un numero crescente di pazienti critici, spesso con insufficienza multi organo, senza una stretta necessità di ricovero in Unità di Terapia Intensiva, ma con criticità che non

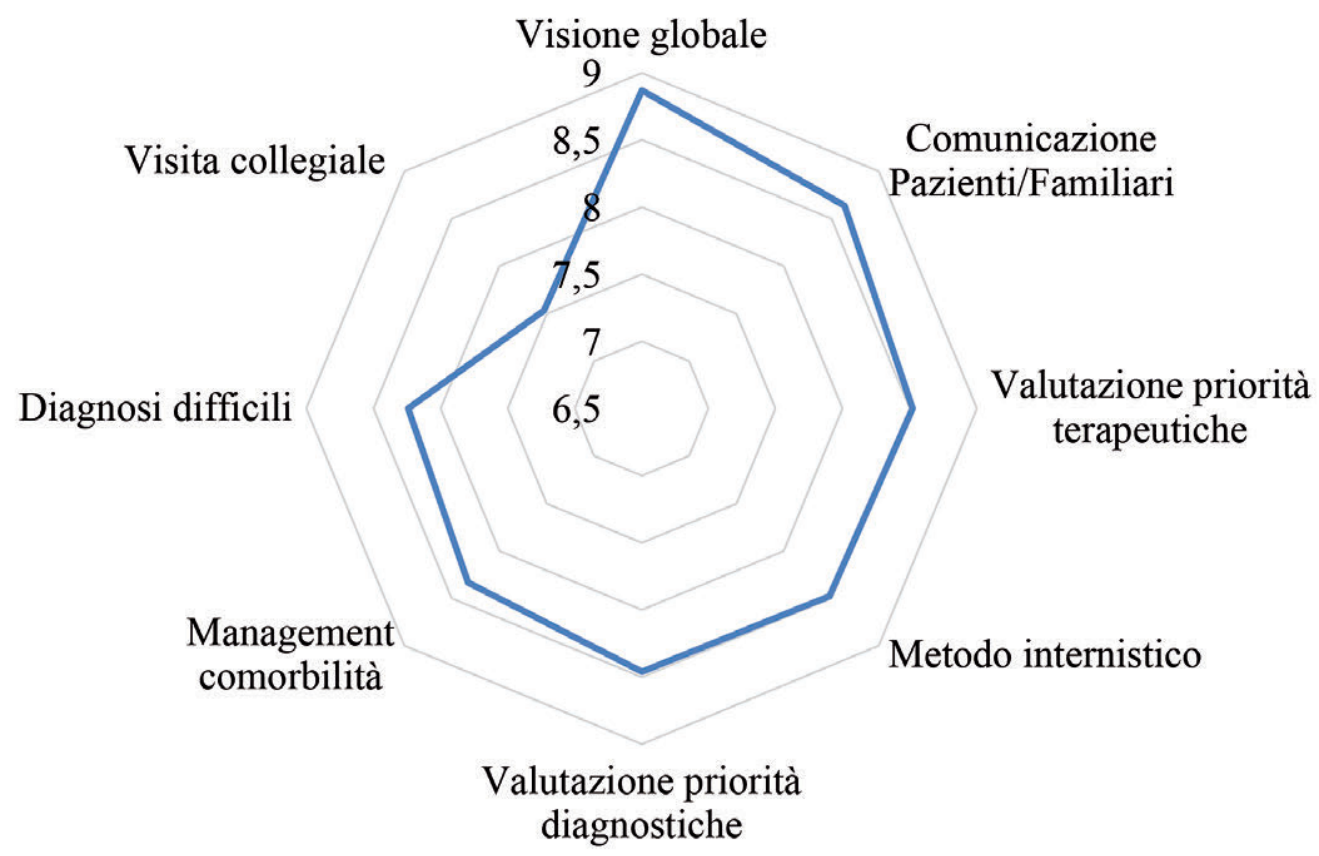

Figura 4. Gli aspetti peculiari dell'attività. Concentrazione degli item prevalentemente nella fascia di voto 8 (punteggio medio 8,38). Il punteggio più alto è stato assegnato all'item Visione globale $(8,87)$, il punteggio più basso all'item Visita collegiale $(\mathbf{7 , 5 3 )}$.

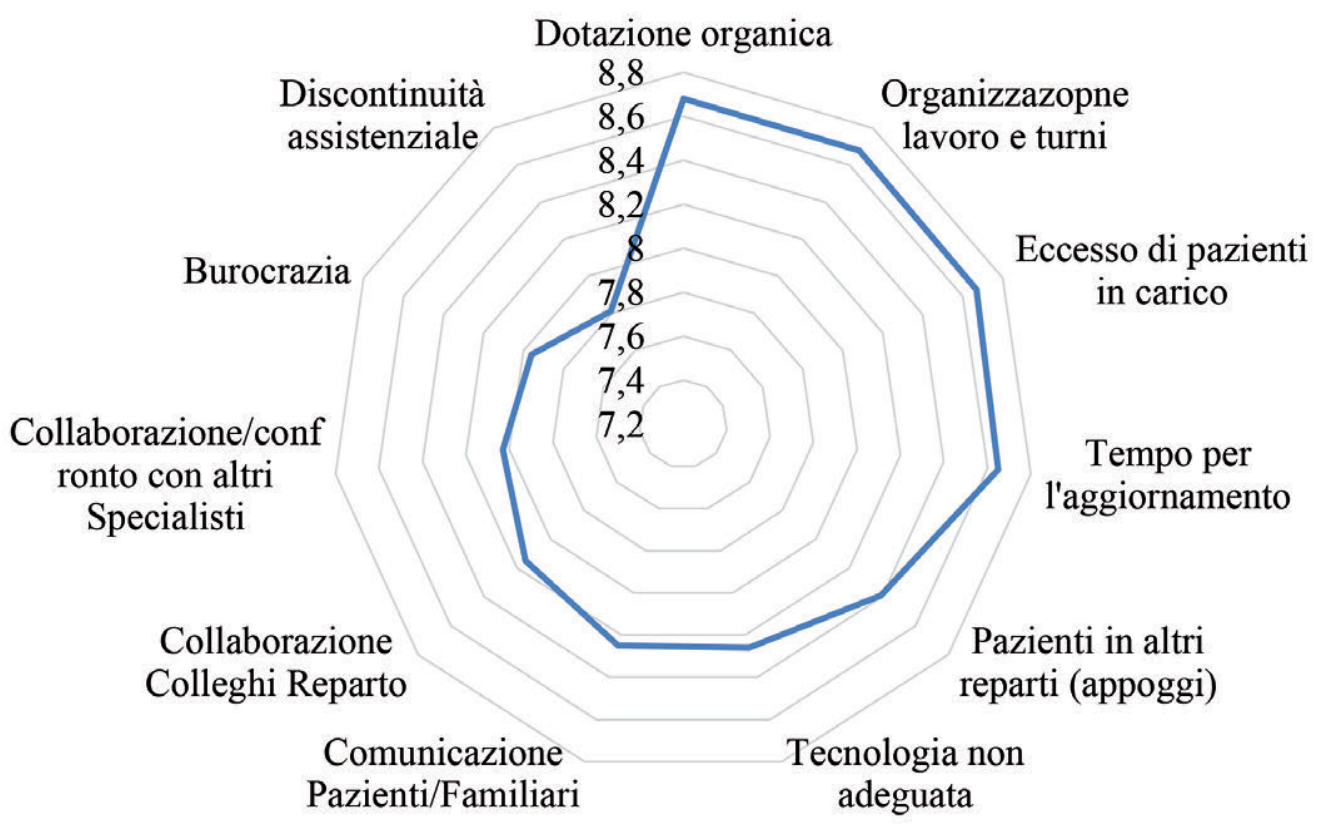

Figura 5. Gli aspetti dell'attività da modificare. Concentrazione degli item prevalentemente nella fascia di voto 8 (punteggio medio 8,32). Il punteggio più alto è stato assegnato all'item Dotazione organica $(8,68)$, il punteggio più basso all'item Discontinuità assistenziale $(7,81)$. 
Tabella 4. Questionario B. Cura dei pazienti critici (n. 662 risposte).

\begin{tabular}{|c|c|c|}
\hline Cura dei pazienti critici & Sì & No \\
\hline Disponi già di posti letto monitorizzati? & $41 \%$ & $59 \%$ \\
\hline I letti monitorizzati possono essere un vantaggio per l'attività? & $93 \%$ & $7 \%$ \\
\hline Sei favorevole all'Internista nella gestione di pazienti critici e instabili? & $84 \%$ & $16 \%$ \\
\hline Sei pronto o favorevole a formarti sulla gestione del paziente critico in Medicina Interna? & $93 \%$ & $7 \%$ \\
\hline
\end{tabular}

Tabella 5. Questionario C. Rapporti con l'Ospedale (A) (n. 647 risposte).

\begin{tabular}{|c|c|c|}
\hline Rapporti con l'Ospedale (A) & Sì & No \\
\hline L'organizzazione della tua UO fornisce una visione forte della Medicina Interna? & $57 \%$ & $43 \%$ \\
\hline Gli atti e le decisioni della Direzione Strategica tengono in giusto conto l'attività che svolgi? & $29 \%$ & $71 \%$ \\
\hline $\begin{array}{l}\text { Nel caso di competenza nosologica dubbia viene chiesto il tuo parere ed esso viene considerato quanto quello } \\
\text { degli specialisti d'organo? }\end{array}$ & $57 \%$ & $43 \%$ \\
\hline
\end{tabular}

UO, Unità Operativa

Tabella 6. Questionario C. Rapporti con l'ospedale (B) (n. 647 risposte).

\section{Rapporti con l'Ospedale (B)}

Come percepisci il tuo ruolo di Internista nell'Ospedale

\begin{tabular}{cc} 
Importante & Ancillare \\
$75 \%$ & $25 \%$ \\
Spesso & Raramente \\
$48 \%$ & $52 \%$ \\
\hline
\end{tabular}

Nell'ultimo anno i percorsi/protocolli validati dalla DS hanno previsto presenza Internista e/o riguardano patologie di interesse internistico?

Importanti Strumentali

Le richieste di tue consulenze sono:

per il percorso per soluzioni contingenti del paziente o logistiche

$82 \% \quad 18 \%$

DS, Direzione Sanitaria

Tabella 7. Questionario C. Rapporti con il DEA (n. 647 risposte).

\begin{tabular}{lccc}
\hline Rapporti con il DEA & & & \\
\hline Prima di un ricovero nella tua UO il PS ti chiama in consulenza? & Sempre & Mai & Raramente \\
& $17 \%$ & $51 \%$ & $32 \%$ \\
\hline Gli Specialisti d'organo sono chiamati in consulenza prima di un ricovero? & Sempre & Mai & Raramente \\
& $67 \%$ & $6 \%$ & Poco \\
\hline Quanto ritieni importante accordi con PS per flusso ricoveri o consulenza strutturata & Molto & $6 \%$ & - \\
in emergenze/urgenze di alcune patologie? & $94 \%$ & No & In minima parte \\
\hline In linea generale i ricoveri presso la tua UO sono appropriati per la Medicina Interna & Sì & $4 \%$ & $3 \%$ \\
tenendo conto di aree di degenza anche nel DEA (Degenza breve, Breve Osservazione, & $63 \%$ & &
\end{tabular}

DEA, Dipartimento Emergenza Accettazione; Holding Area, Area di Attesa; OBI, Osservazione Breve Intensiva; PS, Pronto soccorso; UO, Unità Operativa.

Tabella 8. Differenze fra i punteggi attribuiti ad alcuni indicatori dai Direttori e dagli altri medici della UO.

\begin{tabular}{lcc}
\hline Item & Direttori & Altri medici \\
\hline Presenza di Direttore U.O.C. nelle attività cliniche & 8,4 & 7,95 \\
\hline Degenza media & 7,14 & 6,86 \\
\hline Numero dimissioni l'anno & 7,44 & 6,95 \\
\hline Peso DRG generati & 7,88 & 6,79 \\
\hline Re ricoveri entro 1 mese & 7,68 & 7,9 \\
\hline
\end{tabular}


consentono una adeguata gestione in regime di degenza ordinaria. Obiettivo della Medicina Interna deve essere anche quello di intercettare questo tipo di pazienti attraverso la disponibilità strutturata di tecnologia sufficiente a: i) assicurare l'appropriatezza delle cure; ii) evitare i ricoveri non appropriati sia in ambienti ad alto costo che in ambienti a bassa intensità di cure; iii) inserire a pieno titolo la Medicina Interna nell’organizzazione per Intensità di Cure.

\section{Rapporti con l'ospedale e il DEA}

Le risposte sono riportate nelle Tabelle 5, 6 e 7 .

Benché il 57\% degli Internisti ritenga che la propria UO fornisca una visione forte della MI e percepisca il proprio ruolo come importante (75\%) e non ancillare (25\%), il $71 \%$ ritiene che gli atti della Direzione Strategica non tengono in giusto conto l'importanza dell'attività delle UO di MI riportando così una evidente distanza fra Direzione Strategica e Medici delle UO di MI nella percezione dell'importanza strategica dell'attività complessiva svolta dalle MI negli ospedali italiani. Risulta anche un insufficiente coordinamento fra le attività di MI e l'attività di ricovero di DEA e PS che, a differenza di quanto sembra accadere per i ricoveri nelle UO di specialità d'organo, concordano con gli Internisti un ricovero nella loro UO solo in una minoranza di casi. Ciò potrebbe essere una delle cause della quota di ricoveri inappropriati o solo in minima parte appropriati riportata dagli Internisti che hanno aderito alla survey.

\section{Conclusioni}

Le risposte ad una survey, con tutti i loro limiti intrinseci, certamente esprimono un orientamento, soprattutto quando sono concordanti. Le opinioni di coloro che svolgono un ruolo attivo nelle attività cliniche può essere molto importante per una società scientifica che, come FADOI, si propone di intervenire nell'evoluzione della MI.

Nelle UO di MI a volte non è presente la stessa omogeneità formativa e organizzativa che contraddistingue di solito le specialità d'organo. L'evidente concordanza delle risposte a questa survey, assolutamente non scontata, lascia intravedere una specialità più omogenea dell'atteso nonostante la diversità della formazione post-laurea, dei percorsi professionali, del tipo di ospedale e della sua localizzazione geografica. Una possibile spiegazione potrebbe essere la condivisione degli aspetti fondanti della MI che contaminano l'eterogeneità, minimizzano le differenze iniziali e aggregano sui principi.

Per ciò che riguarda gli indicatori della qualità della cura in MI, l'orientamento degli Internisti è a favore degli indicatori associati alle risorse e alla organizzazione delle attività cliniche piuttosto che verso gli indicatori di carattere prevalentemente amministrativo di solito proposti dalle Direzioni e dai decisori istituzionali delle politiche sanitarie. Il dato, anche in questo ambito, conferma una certa distanza fra medici e gestori, i primi più orientati all'azione che alla pianificazione e con una convinta considerazione dell'autonomia professionale nella organizzazione dell'attività clinica. A questo riguardo le differenze fra gli orientamenti dei Direttori di UO e gli altri medici della UO non sono trascurabili (Tabella 8) e meriterebbero forse un ulteriore futuro approfondimento.

Complessivamente in questa survey gli Internisti confermano l'importanza della cura della persona e della complessità clinica con metodo internistico, ma individuano anche come priorità la continuità assistenziale, la comunicazione, la cultura medica del fine vita e la collaborazione con gli Infermieri della UO e gli altri Specialisti.

Una considerazione minore dell'atteso viene data alla visita collegiale e alla presenza nelle attività cliniche del Responsabile della UO. Il dato non è di univoca interpretazione, ma certamente indica la necessità di aggiornare le modalità e gli obiettivi di questo importante strumento di qualità dell'attività dell'Internista ospedaliero. Inoltre, va considerato che l'attuale restrizione degli organici in molti casi non rende possibile una vera visita collegiale e costringe i pochi Internisti in servizio ad un giro visita con poche possibilità di interazione nell'ambito del team.

Risulta molto chiara, invece, la propensione all'ampliamento delle competenze: i) maggiore autonomia nella NIV, indagini ecografiche e procedure; ii) autonomia nella cura del paziente critico.

La formazione e l'aggiornamento su quest'ultimo argomento sono auspicati dalla grande maggioranza degli Internisti.

Le criticità maggiori sembrano essere costituite dai rapporti con le Direzioni Strategiche e il DEA. Infatti, le risposte alla survey evidenziano: i) una significativa distanza fra gli atti delle Direzioni Sanitarie e i Medici delle UO di MI nella percezione dell'importanza strategica della Medicina Interna e della sua attività complessiva negli ospedali italiani (Tabella 6); ii) un insufficiente coordinamento fra le attività di MI e l'attività di ricovero di DEA e PS (Tabella 7). Questo diffuso fenomeno potrebbe essere una delle cause della quota di ricoveri solo in minima parte appropriati (34\%) o inappropriati (3\%) riportata dagli Internisti.

I dati di questa survey potrebbero essere utili a orientare le iniziative societarie nelle attività formative e nel confronto con le istituzioni.

\section{Ringraziamenti}

Area organizzativa FADOI Lazio: Giuseppe Campagna, Maria Serena Fiore, Fabrizio Tiratterra, Rug- 
gero Pastorelli, David Terracina, Claudio Santini, Annachiara Crespi, Clinical Project Manager, Centro Studi FADOI.

\section{Bibliografia}

1. Silingardi M. C'è bisogno di una nuova figura professionale? Quaderni Ital J Med 2018;3:9-12.

2. Paladini L, Hodder R, Bellia V, et al. Physician specialty as a source of heterogeneity in the care of patients with COPD. Chest 2011;140:1666-7.

3. Antonelli Incalzi R. An epidemiological overview and clinical picture of COPD in the elderly. JGG 2016;64: 119-25.

4. Cranston M, Semple C, Duckitt R, et al. The practice of internal medicine in Europe: organisation, clinical conditions and procedures. Eur J Intern Med 2013;24:627-32.

5. Berti F. La carta della qualità in Medicina Interna. Ital J Med 2010;4:207-11.

6. Orlandini F. Analisi dei modelli esistenti: future hospital program. Quaderni Ital J Med 2014;3:13-7.

7. Future Hospital Commission. Future Hospital: caring for medical patients. A report from the Future Hospital Commission to the Royal College of Physicians. London: Royal College of Physicians; 2013.

8. Adler M, Zilio E. Gazing into the Oracle: The applications of Delphi Method to Social Policy and Public Health. London: Kingsley Publishers; 1996.

9. Bertin G. Un modello di valutazione basato sul giudizio degli esperti. Milano: Edizioni Etas libri; 1994.

10. De Carli S. È ancora possibile una reale alleanza fra clinici e gestori per una Medicina Interna ospedaliera sostenibile? Quaderni Ital J Med 2014;2:95-100.

11. Foglia E, Croce D. Indicatori della qualità nella Medicina Interna Ospedaliera: solamente durata della degenza, ricoveri inappropriati, mortalità intraospedaliera e tasso di re-ricovero a 30 giorni? Quaderni Ital J Med 2014;2:106-13.

12. Mc Glynn EA, Schneider EC, Kerr E. Remaiging Quality Measurament. N Engl J Med 2014;371:2150-52.

13. Pannick S, Watchter RM, Vincent $C$, et al. Rethinking medical ward quality. BMJ 2016;355:i5417.

14. Sabbatini AK, Wright B. Excluding Observation Stays from Readmission Rates. What Quality Measures are Missing. N Engl J Med 2018;378:2062-3 .

15. Gupta A, Allen LA, Bhatt DL, et al. Association of the Hospital Readmission Reduction Program Implementation with readmission and mortality outcomes in heart failure. JAMA Cardiol 2018;3:44-53.

16. Finkelstein A, Zhou A, Taubman S, et al. Health Care Hotspotting - A Randomized, Controlled Trial. N Engl J Med 2020;382:152-62.

17. Simonetti V, Comparcini D, Tesei L, et al. Il giro visita: una prassi che garantisce la qualità? Una revisione integrativa della letteratura. Riv L'infermiere 2019:6.

18. Royal College of Physicians, Royal College of Nursing. Ward rounds in medicine: principles for best practice. London: Royal College of Physicians; 2012.

19. Gilliand N, Catherwood N, Chen S, et al. Ward round template: enhancing patient safety on ward rounds 2018. BMJ Qual Improv Rep 2018;7:e000170.

20. Gonzalo JD, Kuperman E, Lehman E, Haidet P. Bedside interprofessional rounds: perceptions of benefits and barriers by internal medicine nursing staff, attending physicians, and housestaff physicians. J Hosp Med 2014;9:646-51. 


\title{
Gli indicatori della qualità dell'assistenza nelle Unità Operative di Medicina Interna secondo gli Internisti FADOI
}

\author{
Maria Serena Fiore, ${ }^{1}$ Fabrizio Tiratterra ${ }^{2}$ \\ ${ }^{1}$ Medicina Interna, Ospedale Sandro Pertini, ASL Roma 2; ${ }^{2}$ Medicina Interna, Ospedale Giovanni Battista Grassi, ASL Roma 3, \\ Roma, Italia
}

\section{Introduzione}

FADOI ha recentemente condotto una Survey webbased denominata Un giorno in Medicina. Un viaggio attraverso le opinioni degli Internisti FADOI che ha esplorato, tra i diversi argomenti, le opinioni degli internisti circa gli indicatori ritenuti più idonei per valutare la qualità delle cure in una Unità operativa complessa di Medicina Interna. Gli indicatori scelti ed analizzati sono stati suddivisi in quattro categorie: statistico-amministrativi, correlati ad organizzazione e risorse, correlati ai processi, correlati agli esiti. Gli internisti FADOI hanno attribuito i punteggi più elevati agli indicatori riferiti a: mantenimento della continuità assistenziale (presenza del medico 5 giorni a settimana), adeguato rapporto infermiere/paziente e medico/paziente, condivisione degli aspetti clinico-terapeutici, soddisfazione del paziente, possibilità di eseguire autonomamente e direttamente prestazioni strumentali diagnostico-terapeutiche. I più classici indicatori di performance (degenza media, mortalità, peso dei DRG) hanno ottenuto invece una valutazione sensibilmente più bassa. L'alta partecipazione alla Survey ha dimostrato l'interesse degli internisti ospedalieri a riflettere sugli elementi caratterizzanti la Medicina Interna nell'attuale scenario della Sanità.

Il tema della qualità, nelle sue dimensioni professionale, organizzativo-gestionale, percepita e sociale, è negli ultimi anni largamente esplorato in Medicina di pari passo con lo sviluppo della Clinical Governance;

Corrispondente: Maria Serena Fiore, Medicina Interna, Ospedale Sandro Pertini, ASL Roma 2, via dei Monti Tiburtini 385, 00157 Roma, Italia.

Tel.: +39.06.4143.6598.

E-mail: mariaserenafiore@gmail.com

Articolo pubblicato secondo la Creative Commons Attribution NonCommercial 4.0 License (CC BY-NC 4.0).

${ }^{\circ}$ Copyright: the Author(s), 2020

Licensee PAGEPress, Italy

QUADERNI - Italian Journal of Medicine 2020; 8(3):14-19 tuttavia non sempre il termine qualità è declinato operativamente con la precisazione di uno specifico obiettivo (es., valutare, standardizzare, migliorare ...).

In linea generale perseguire qualità significa: i) individuare opportunità di miglioramento e definire, per rendere le stesse effettive, progetti di valutazione e di intervento (tra questi particolare rilievo assumono i percorsi assistenziali); ii) definire e condividere procedure organizzative che siano basate su una analisi attenta dei processi e contribuiscano alla loro ottimizzazione; iii) definire, condividere ed implementare Linee guida (intese come sequenze di raccomandazioni che riguardano comportamenti professionali, basate il più possibile sulle evidenze scientifiche) da contestualizzare nei percorsi assistenziali; iv) curare che le procedure, le Linee guida ed i percorsi assistenziali siano costantemente aggiornati sulla base delle acquisizioni tecnologiche, dei dati emergenti dalla letteratura scientifica nonché dalle indicazioni provenienti dalla verifica della loro applicazione; v) definire ed attuare sistemi di monitoraggio (sia degli eventi avversi che dei livelli di soddisfazione di pazienti e familiari) in modo da individuare nuove opportunità di miglioramento e pianificare le azioni conseguenti nell'ottica e secondo le modalità del Miglioramento Continuo di Qualità (MCQ). ${ }^{1-5}$

La qualità dell'assistenza è necessariamente il risultato dell'interazione tra le persone (che esprimono qualità professionale) ed il sistema organizzativo (qualità organizzativa) ed è quindi una proprietà dell'intero sistema di assistenza che non si esaurisce nel miglioramento della capacità professionale dei singoli operatori.

La qualità professionale assicura che ogni intervento sia coerente con le migliori conoscenze scientifiche e venga applicato con le necessarie abilità tecniche. Essa si esprime come Efficacia attesa (capacità di un certo intervento di modificare in modo favorevole le condizioni di salute delle persone a cui è rivolto, quando applicato in condizioni ottimali), Appropriatezza (utilità della prestazione per la persona cui è applicata) ed Efficacia pratica (risultati ottenuti dall'applicazione di routine dell'intervento).

È ben noto, inoltre, che la misura della qualità pro- 
fessionale sia basata sull'analisi delle risorse utilizzate per assicurare l'assistenza (input), delle procedure attraverso le quali la stessa viene assicurata (processi), delle prestazioni erogate (output) e dei benefici ottenuti dai pazienti (outcomes).

In questo contesto, alla necessità di misurare (cioè determinare con strumenti adeguati ed appositi la quantità o l'estensione di un fenomeno o di una grandezza) è evidentemente associata quella di valutare e cioè di accertare il valore di un intervento attraverso la comparazione tra dati predefiniti e dati raccolti $a d$ hoc; solo in tal modo è infatti possibile comprendere le cause di un determinato fenomeno e/o prendere le decisioni più corrette ed informate. ${ }^{6}$

\section{Indicatori}

Uno strumento essenziale per perseguire e mantenere la qualità è rappresentato quindi dalla disponibilità di misurazioni, capaci di monitorare l'attività in corso e di orientare i cambiamenti. Berwick (1996) sosteneva che misurare è indispensabile perché nell'atto di definire le misure si chiariscono gli obiettivi e perché le misure sono indispensabili per decidere se continuare a fare quello che si fa o se cambiare. Le misure sono anche indispensabili per valutare se vale la pena di introdurre un'innovazione.

Gli indicatori sono gli strumenti attraverso i quali è possibile misurare.

Per indicatore si intende una variabile, ad alto contenuto informativo, utilizzata per descrivere in modo sintetico un fenomeno complesso e capace di fornire (attraverso il confronto con uno standard o valore soglia) elementi necessari per prendere decisioni e avviare iniziative di miglioramento.

Gli indicatori quindi, non misurano direttamente la qualità, ma rappresentano dei segnali che dirigono l'attenzione verso aspetti delle cure destinati ad essere approfonditi.

Possiamo quindi, sinteticamente attribuire ad essi diversi scopi: i) fare confronti (tra professionisti, strutture, nel tempo ...); ii) esprimere valutazioni comparative con standard di riferimento; iii) individuare priorità; iv) valutare attività e servizi in termini di raggiungimento di risultati attesi; v) misurare l'efficienza; vi) ed anche orientare gli utenti nelle scelte di cura. ${ }^{6-9}$

Gli indicatori debbono possedere alcuni necessari requisiti che derivano da caratteristiche intrinseche (riproducibilità, accuratezza, pertinenza) e da caratteristiche estrinseche, cioè legate alla rilevazione ed all'uso (completezza, misurabilità, accettabilità, utilità).

Riproducibilità: grado di accordo nel misurare lo stesso fenomeno, tra più osservatori o tra lo stesso osservatore in più occasioni.

Accuratezza: grado di accordo tra il valore osservato e quello vero.
Pertinenza: capacità di rappresentare il fenomeno che si intende valutare.

Completezza: $\mathrm{n}$. di dati persi.

Misurabilità e accettabilità: possibilità di ottenere, nella pratica corrente, $i$ dati necessari alla elaborazione dell'indicatore.

Utilità: capacità di influenzare le decisioni.

Un buon indicatore deve essere quindi (utilizzando l'acronimo MISURADOC): Misurabile (rilevabile in modo riproducibile ed accurato), Importante (pertinente ad un problema frequente o con notevoli conseguenze sui pazienti o sui costi), Semplice, Utilizzabile (preferibilmente accompagnato da valori soglia o standard), Risolvibile (relativo ad un problema per cui si può fare qualcosa con le risorse disponibili), Discriminante (capace di distinguere tra realtà diverse e di mettere in luce i cambiamenti nel tempo), Ottenibile (rilevabile con costi e tempi accettabili), Completo (rilevabile e rilevato in tutti gli eventi /tutti i soggetti in cui lo si deve rilevare). ${ }^{6}$

In Medicina vi è da tempo un ampio uso degli indicatori di output soprattutto da parte dei gestori della Sanità con l'obiettivo di valutare le performance.( es. degenza media, re-ricovero a 30 giorni, indice di rotazione di posto letto ...) e per tale motivo essi sono centrati su criteri economico-gestionali. Successivamente è comparsa l'esigenza di valutare non solo la performance ma anche gli esiti (outcome) per cui sono stati sviluppati sistemi centrati su indicatori di esito (come accade ad esempio nel P.Re.Val.E, Programma regionale valutazione Esiti del Lazio) validi soprattutto per le branche specialistiche. Non vi sono infatti indicatori specifici per la Medicina Interna capaci di descrivere e valutare compiutamente le attività richieste nelle unità operative di Medicina per pazienti complessi e multipatologici. ${ }^{10-17}$

\section{Analisi dei dati della survey}

Nella Survey Un giorno in Medicina. Un viaggio attraverso le opinioni degli Internisti FADOI, sono stati selezionati e compresi in un questionario a 31 items gli elementi utili ad indagare gli aspetti percepiti come di qualità per l'assistenza da parte degli internisti ospedalieri e sulla base di questo definiti Indicatori. A ciascuno degli indicatori è stato attribuito un valore numerico da 1 (Irrilevante) a 10 (Estremamente importante). Sono state fornite complessivamente 740 risposte complete.

Per le loro caratteristiche, gli elementi presi in considerazione possono essere sostanzialmente suddivisi in quattro gruppi: i) Indicatori statistico-amministrativi correlati all'attività (Degenza media, Percentuale ricoveri dal Pronto Soccorso, Numero dimissioni l'anno, Numero di pazienti appoggiati in altro Reparto, Numero di pazienti in letti aggiunti in Reparto, Percentuale di mortalità, Percentuale di dimissioni a 
domicilio, Numero di re-ricoveri entro un mese, Peso DRG generati); ii) Indicatori correlati ad organizzazione e risorse (Individuazione del Medico che prende in carico il paziente, Presenza del Direttore di UO nelle attività cliniche, Presenza in visita di uno stesso Medico per almeno 5 giorni/settimana, Presenza dello stesso infermiere nella visita quotidiana, Percentuale ore di servizio svolte dai Medici in attività di guardia, Utilizzo di lettera di dimissione strutturata, Possibilità di controlli ambulatoriali post-dimissione, Rapporto n. medici/n. pazienti, Rapporto n. infermieri/n. pazienti); iii) Indicatori correlati ai processi (Meeting clinici e discussioni collegiali, Organizzazione per continuità assistenziale, Percorsi diagnostico-terapeutici strutturati e condivisi, Gestione rischio clinico, Possibilità di effettuare direttamente esami strumentali ecografici e procedure, Possibilità di effettuare NIV (ventilazione non invasiva.), Gestione del fine vita); iv) Indicatori correlati agli esiti (Grado di soddisfazione dei pazienti, Frequenza insorgenza delle lesioni da pressione, Numero delle cadute dei pazienti, $\mathrm{Nu}-$ mero dei contenziosi, Percentuale di pazienti sottoposti a cateterismo vescicale, Percentuale di mortalità).

I punteggi espressi per i diversi gruppi di indicatori sono esemplificati nelle Tabelle 1-4.

Il gruppo di indicatori in Tabella 1, prettamente di output, ha ottenuto il punteggio medio più basso tra $\mathrm{i}$ quattro; in particolare Degenza media, Mortalità e Peso dei DRG, gli indicatori di esito più frequenti e più comunemente utilizzati nei cicli di gestione della performance (budget), risultano aver ottenuto il minor punteggio in termini di rilevanza da parte degli internisti ospedalieri.

Gli indicatori in Tabella 2 presentano un indice di gradimento tra gli internisti decisamente superiore ai precedenti infatti il punteggio medio è sensibilmente più alto.

Ciò dimostra che gli internisti ben comprendono la centralità della qualità organizzativa per garantire una Sanità di qualità ai cittadini. In particolare, le ri-

Tabella 1. Questionario A: indicatori di qualità della cura (n. 740 risposte).

\begin{tabular}{lc}
\hline Indicatori statistico-amministrativi correlati all'attività & 6,99 \\
\hline Degenza media & 7,50 \\
\hline Percentuale ricoveri dal Pronto Soccorso & 7,07 \\
\hline Numero dimissioni l'anno & 7,24 \\
\hline Numero di pazienti appoggiati in altro Reparto & 7,18 \\
\hline Numero di pazienti in letti aggiunti in Reparto & 6,87 \\
\hline Percentuale di mortalità & 7,25 \\
\hline Percentuale di dimissioni a domicilio & 7,85 \\
\hline Numero di re-ricoveri entro un mese & 7,05 \\
\hline Peso DRG generati & $\mathbf{7 , 2 2}$ \\
\hline Punteggio medio & \\
\hline
\end{tabular}

DRG, diagnosis-related group o raggruppamento omogeneo di diagnosi.

Tabella 2. Questionario A: indicatori di qualità della cura (n. 740 risposte).

\begin{tabular}{ll}
\hline Indicatori correlati a organizzazione e risorse & 7,86 \\
\hline Individuazione del Medico che prende in carico il paziente & 8,06 \\
\hline Presenza del Direttore UO nelle attività cliniche & 8,58 \\
\hline Presenza in visita di uno stesso Medico per almeno 5 giorni/settimana & 7,58 \\
\hline Presenza dello stesso infermiere nella visita quotidiana & 7,64 \\
\hline Percentuale ore di servizio svolte dai Medici in attività di guardia & 8,08 \\
\hline Utilizzo di lettera di dimissione strutturata & 8,28 \\
\hline Possibilità di controlli ambulatoriali post-dimissione & 8,08 \\
\hline Rapporto n. medici/n. pazienti & 8,98 \\
\hline Rapporto n. infermieri/n. pazienti & $\mathbf{8 , 1 2}$ \\
\hline Punteggio medio &
\end{tabular}

UO, Unità Operativa. 
sposte fornite evidenziano da un lato la rilevanza di un adeguato rapporto tra pazienti e personale sanitario (medico ed infermieristico) e dall'altra l'importanza della presa in carico continuativa del paziente (presenza di uno stesso medico in visita per almeno 5 giorni a settimana e presenza del Direttore di UO nelle attività cliniche) da parte di un medico.

In merito ai Processi, che comunque mantengono un elevato indice di considerazione da parte degli internisti, vi è una particolare attenzione dedicata all'approfondimento condiviso a livello di equipe degli aspetti clinico-diagnostici (Tabella 3).

Un dato parzialmente sorprendente è il livello di importanza attribuito alla gestione del Fine vita a testimoniare l'impegno diffuso della Medicina Interna ospedaliera su questa emergente tematica.
Infine, risulta avere un importante livello di gradimento la possibilità di utilizzare, in modo autonomo e diretto all'interno della UO di Medicina, strumenti tecnologici diagnostico-terapeutici quali ecografia e NIV.

I punteggi attribuiti dagli internisti agli indicatori scelti come correlati agli esiti (outcomes) nella Survey dimostrano un gradimento intermedio (Tabella 4). Tra questi, quello più largamente valutato è il grado di soddisfazione dei pazienti e questo è un elemento che conferma l'orientamento degli internisti ospedalieri per una attività clinica di qualità.

Il 23,5\% dei partecipanti alla Survey svolgeva il ruolo di Direttore di UO di Medicina Interna. Le risposte prodotte dai Direttori sono risultate sostanzialmente sovrapponibili a quelle dei Dirigenti medici ad eccezione di alcuni indicatori esemplificati in Tabella 5. Si

Tabella 3. Questionario A: indicatori di qualità della cura (n. 740 risposte).

\begin{tabular}{lc}
\hline Indicatori correlati ai processi & 8,45 \\
\hline Meeting clinici e discussioni collegiali & 8,02 \\
\hline Organizzazione per continuità assistenziale & 8,35 \\
\hline Percorsi diagnostico-terapeutici strutturati e condivisi & 8,00 \\
\hline Gestione rischio clinico & 8,39 \\
\hline Possibilità di effettuare direttamente esami strumentali ecografici e procedure & 8,25 \\
\hline Possibilità di effettuare NIV (ventilazione non invasiva) & 8,5 \\
\hline Gestione del fine vita & $\mathbf{8 , 2 8}$ \\
\hline Punteggio medio &
\end{tabular}

Tabella 4. Questionario A: indicatori di qualità della cura (n. 740 risposte).

\begin{tabular}{ll}
\hline Indicatori correlati agli esiti & \\
\hline Grado di soddisfazione dei pazienti & 8,24 \\
\hline Frequenza insorgenza delle lesioni da pressione & 8,04 \\
\hline Numero delle cadute dei pazienti & 7,40 \\
\hline Numero dei contenziosi & 7,01 \\
\hline Percentuale di pazienti sottoposti a cateterismo vescicale & 6,71 \\
\hline Percentuale di mortalità & 6,87 \\
\hline Punteggio medio & $\mathbf{7 , 3 7}$ \\
\hline
\end{tabular}

Tabella 5. Differenze fra punteggi attribuiti ad alcuni indicatori dai Direttori e dagli altri Medici.

\begin{tabular}{lcc}
\hline Indicatore & Direttori & Altri \\
\hline Presenza di Direttore UO nelle attività cliniche & 8,4 & 7,95 \\
\hline Degenza media & 7,14 & 6,86 \\
\hline Numero dimissioni l'anno & 7,44 & 6,95 \\
\hline Peso DRG prodotti & 7,88 & 6,79 \\
\hline Re ricoveri entro 1 mese & 7,68 & 7,9 \\
\hline
\end{tabular}


tratta in particolare di 4 indicatori su 5, tutti di performance, e ciò riflette l'attenzione agli aspetti gestionali come caratteristica dell'attività degli internisti Direttori che devono orientare le loro priorità in funzione del raggiungimento degli obiettivi concordati e condivisi con le Direzioni Strategiche. È anche interessante notare, d'altro canto, che i Direttori ritengono comunque importante la loro presenza diretta nello svolgimento delle attività cliniche come dimostrato dalle risposte date al quesito specifico.

\section{Conclusioni}

Uno degli obiettivi della Survey FADOI Un giorno in Medicina. Un viaggio attraverso le opinioni degli Internisti FADOI è stato quello di sondare l'orientamento dei clinici in merito a quali potrebbero essere gli indicatori più idonei per valutare la qualità della cura in una Unità Operativa di Medicina Interna.

Gli indicatori che più frequentemente compaiono negli obiettivi che vengono assegnati alle UO di Medicina sono sostanzialmente obiettivi di output. Letteralmente l'output è ciò che viene messo fuori, quindi, nel caso delle organizzazioni sanitarie, le prestazioni. ${ }^{18}$ Classici obiettivi di output con cui viene misurata dalle Direzioni Generali di ASL e Aziende Ospedaliere l'attività di degenza ordinaria dei reparti di Medicina Interna sono il numero di dimissioni, la degenza media, il peso medio. ${ }^{19}$

Nel corso dell'ultimo decennio vi è stata una crescente attenzione a livello internazionale verso gli indicatori di esito (o indicatori di outcome), cioè indicatori che tentano di quantificare il miglioramento o il peggioramento dello stato di salute del paziente durante il ricovero. In questa direzione, in Italia, dal 2010 è stato avviato da AGENAS, per conto del Ministero della Salute, il Programma Nazionale Esiti (PNE) per valutare comparativamente efficacia, equità, sicurezza e appropriatezza delle cure prodotte nell'ambito del Servizio sanitario italiano. I risultati dell'edizione 2017 di PNE analizzano 166 indicatori, nessuno di questi è dedicato all'area clinica Medicina Interna. ${ }^{20}$

Non è quindi sorprendente che vi sia una riflessione su come misurare la qualità in Medicina Interna. Sono in molti a credere che sia giunto il tempo di ripensare gli indicatori che possono essere utili per valutare la qualità delle cure in pazienti con multiple patologie croniche. ${ }^{3,11}$

In effetti, lo scenario epidemiologico caratterizzato dal progressivo invecchiamento della popolazione, dall'aumento della prevalenza di persone con malattie croniche e ricorrenti riacutizzazioni, dall' aumento delle persone con decadimento cognitivo, ha drasticamente cambiato la tipologia dei pazienti ricoverati nei reparti di degenza di Medicina Interna.
Anche le scelte di riordino del Sistema Sanitario Nazionale (SSN), che hanno ridefinito il rapporto tra posti letto e popolazione con conseguente sostanziale riduzione dei posti letto per acuti, e le linee di indirizzo regionale orientate a favorire il ricovero nei reparti di Medicina Interna di pazienti provenienti dal Dipartimento Emergenza Accettazione (DEA) hanno esercitato un ruolo nella tipologia dei pazienti che attualmente vengono curati nei nostri reparti.

L'azione congiunta del progressivo invecchiamento della popolazione e quella del riordino del SSN hanno prodotto un effetto divergente. Da un lato, è aumentato il numero di pazienti ricoverati con disabilità e/o malattie croniche in fase avanzata che necessitano di un adeguato percorso di transizione dalla degenza ospedaliera al domicilio, oppure ad una degenza caratterizzata da più bassa intensità di cure, per la cura dei quali è fondamentale la qualità della continuità assistenziale, della modalità di transizione da un livello assistenziale all'altro, della comunicazione con familiari e caregivers. Dall'altro, la riduzione dei posti letto ed il sostanziale aumento del numero di pazienti ricoverati direttamente dal DEA in Medicina Interna, hanno contribuito ad aumentare la quantità di pazienti critici/acuti che necessitano di una più alta intensità di cura, per i quali può essere indicata la monitorizzazione di parametri vitali, il trattamento con ventilazione non invasiva (NIV), l'ecografia bedside.

Tra gli ipotetici indicatori di qualità proposti dalla Survey agli Internisti, quelli che hanno ottenuto il maggior gradimento sono stati: il rapporto $n$. infermieri/n. pazienti, il rapporto n. medici/n. pazienti, la presenza in visita di uno stesso medico per almeno 5 giorni la settimana, meeting clinici e discussioni collegiali, gestione del fine vita, possibilità di effettuare direttamente esami strumentali ecografici e procedure.

Il rapporto $n$. infermieri/n. pazienti e quello n. medici/n. pazienti sono indicatori di struttura, forniscono informazioni sulle caratteristiche strutturali del reparto. È evidente la rilevanza di tali indicatori, infatti nei reparti ad alta intensità di cura (per esempio in quelli di Rianimazione) è chiaramente definito lo standard minimo del rapporto pazienti/infermieri. Nei reparti di Medicina Interna, invece, questo rapporto può variare, come anche quello tra $\mathrm{n}$. pazienti/n. medici. Forse potrebbe essere utile contribuire a definire i valori degli standard minimi assistenziali in termini di dotazione di medici ed infermieri nei reparti di Medicina rapportandoli al numero medio di pazienti con grave disabilità e/o critici che vi vengono ricoverati.

La presenza in visita di uno stesso medico per almeno 5 giorni la settimana ed i meeting clinici e discussioni collegiali sono due elementi che contribuiscono in modo fondamentale per individuare con chiarezza il medico che prende in carico il paziente e per garantire la continuità delle cure nel corso 
della degenza e favorire scelte diagnostico-terapeutiche in linea con le evidenze della letteratura.

La gestione del fine vita e la possibilità di effettuare direttamente esami strumentali ecografici e procedure sono competenze ormai necessarie all'Internista in considerazione delle caratteristiche dei pazienti di cui deve farsi carico.

In conclusione, dalla valutazione del gradimento degli ipotetici indicatori di qualità proposti dalla Survey emerge la consapevolezza degli Internisti del cambiamento epidemiologico che è avvenuto nei reparti e la necessità di adeguarvi la qualità dell'organizzazione e le competenze del clinico.

\section{Bibliografia}

1. Donabedian A. The definition of quality and approaches to its management, vol 1: explorations in quality assessment and monitoring. Ann Arbor, MI: Health Administration Press; 1980.

2. Donabedian A. The quality of care. How can it be assessed? JAMA 1988;260:1743-8.

3. Pannick S. Rethinking medical ward quality. BMJ 2016;355:i5417.

4. Future Hospital Commission. Future hospital: Caring for medical patients. A report from the Future Hospital Commission to the Royal College of Physicians. London: Royal College of Physicians; 2013.

5. American Hospital Association. Reconfiguring the bedside care team of the future. 2013. Available from: http://www.aha.org/content/13/beds-whitepapergen.pdf

6. Morosini P. Indicatori in valutazione e miglioramento della qualità professionale. 2004, v, 112 p. Rapporti ISTISAN 04/29 Rev.

7. Lilford RJ, Brown CA, Nicholl J. Use of process measures to monitor the quality of clinical practice. BMJ 2007;335:648-50.

8. Majeed A, Lester H, Bindman AB. Improving the qua- lity of care with performance indicators. BMJ 2007;335:916-8.

9. Lohr KN, Schroeder SA. A strategy for quality assurance in Medicare. N Engl J Med 1990;322:707-12.

10. Fonarow GC, Abraham WT, Albert NM, et al. Association between performance measures and clinical outcomes for patients hospitalized with heart failure. JAMA 2007;297:61-70.

11. McGlynn EA, Schneider EC, Kerr EA. Reimagining Quality Measurement N Engl J Med 2014;371;23.

12. Kerr EA, Hayward RA. Patient-centered performance management: enhancing value for patients and health care systems. JAMA 2013;310:137-8.

13. Ferrer R, Artigas A, Levy MM, et al. Improvement in process of care and outcome after a multicenter severe sepsis educational program in Spain. JAMA 2008;299:2294-303.

14. Pannick S, Beveridge I, Wachter RM, et al. Improving the quality and safety of care on the medical ward: A review and synthesis of the evidence base. Eur J Intern Med 2014;25:874-87.

15. Barnett K, Mercer SW, Norbury M, et al. Epidemiology of multimorbidity and implications for health care, research, and medical education: across-sectional study. Lancet 2012;380:37-43.

16. Nelson EC, Batalden PB, Huber TP, et al. Microsystems in health care: Part 1. Learning from high-performing front-line clinical units. Jt Comm J Qual Improv 2002;28:472-93.

17. Wong BM, Dyal S, Etchells EE, et al. Application of a trigger tool in near real time to inform quality improvement activities: a prospective study in a general medicine ward. BMJ Qual Saf 2015;24:272-81.

18. Beccastrini S, Gardini A, Tonelli S. "Piccolo dizionario della Qualità". Centro Scientifico Editore; 2001.

19. Foglia $\mathrm{E}$, Croce D. Indicatori di qualità nella Medicina Interna ospedaliera: solamente durata di degenza, ricoveri inappropriati, mortalità intraospedaliera e tasso di re-ricovero a 30 giorni? Q Ital J Med 2014;2:106-13.

20. Davoli M, Basiglini A. Indicatori PNE: istruzioni per l'uso Monitor numero 43-2018 pag 13-27. 


\title{
L'attività clinica nelle Unità Operative di Medicina Interna: gli aspetti gratificanti e quelli da modificare
}

\author{
David Terracina, ${ }^{1}$ Giuseppe Campagna ${ }^{2}$ \\ ${ }^{1}$ UOC di Medicina, Ospedale S. Eugenio, Roma; ${ }^{2}$ UOC di Medicina, Ospedale S. Maria Goretti, Latina, Italia
}

\section{Introduzione}

La Medicina Interna è una branca del sapere medico molto antica il cui ambito di interesse, oltremodo ampio, è divenuto nel corso delle ultime quattro decadi estremamente eterogeneo, parallelamente all'espansione, sempre crescente, delle conoscenze che ha condotto alla nascita di ambiti sub-specialistici confinati a patologie d'organo o di apparato o, addirittura, a singole malattie. La gemmazione dalla Medicina Interna di un'ampia gamma di sub-specialità ha, inevitabilmente condotto alla parcellizzazione del sapere medico oscurando, almeno inizialmente, la Disciplina Madre il cui ambito lavorativo è divenuto meno evidente e meno rappresentativo anche per il numero esiguo di Internisti che, nello stesso lasso di tempo, sono stati formati dalle Università Italiane. Nelle speranze ed ambizioni di un neolaureato, con alle spalle due-tre anni di frequenza in qualità di studente interno nella Clinica Medica, c'era l'ingresso alla Scuola di Specializzazione in Medicina Interna ma ciò era riservato a soli 3-5 iscritti all'anno in ogni Università, con la necessità molto concreta di dover optare per un'altra subspecialità, l'equivalente di una bocciatura sul campo. Peraltro le campagne di promozione di alcune subspecialità sono state molto aggressive tanto che nei ricordi di studente universitario alcuni possono rammentare la richiesta di adesione, in sede di esame, a una dichiarazione preparata ad hoc su ciclostilato

Corrispondente: David Terracina, UOC di Medicina Ospedale Sant'Eugenio, Roma, Italia

E-mail: david.terracina2@gmail.com ; campagna.casa@libero.it

Articolo pubblicato secondo la Creative Commons Attribution NonCommercial 4.0 License (CC BY-NC 4.0).

${ }^{\circ}$ Copyright: the Author(s), 2020

Licensee PAGEPress, Italy

QUADERNI - Italian Journal of Medicine 2020; 8(3):20-28 con la quale si affermava di essere favorevoli all'apertura, negli Ospedali, di reparti specialistici, p.e. di Malattie Infettive. Nonostante questo viaggio alquanto travagliato la Medicina Interna è rimasta insostituibile perché concede spazio ad un inquadramento unitario del paziente e a una diagnosi tempestiva in grado di rendere puntuale il percorso terapeutico e l'ambito lavorativo, è un luogo eccezionale di confronto con la mission non di dimostrare ma di curare, non di prescrivere tanti esami ma di optare se fare o meno un esame. Negli ultimi decenni si è però progressivamente instaurato un diffuso clima di insoddisfazione e di precarietà in Italia. Nonostante gli evidenti progressi ed i grandi risultati raggiunti dalla medicina, questo clima è riuscito a coinvolgere anche il medico, facendo venir meno quella forte motivazione che è stata a ognuno necessaria per intraprendere i lunghi e difficili studi. Il pessimismo con cui si valuta l'attività porta al continuo evidenziare le criticità del sistema, trascurando le opportunità, gli aspetti positivi ed i punti di forza dello sviluppo della nostra professione, trasmettendo l'idea che il miglioramento dell'attività lavorativa sia correlato unicamente all'individuazione e risoluzione dei problemi tralasciando ciò che genera soddisfazione, entusiasmo e comportamenti virtuosi. ${ }^{1}$ L'attuale legislazione ha reso la Laurea in Medicina e Chirurgia non spendibile all'interno dl Servizio Sanitario Nazionale, richiedendo un ulteriore percorso di specializzazione per poter partecipare ai concorsi e agli avvisi. Il risultato è che pochi fortunati riescono ad inserirsi stabilmente in ambiente lavorativo non prima dei 28-29 anni di età, ma non è raro che si debba attendere anche i 40 anni e oltre. Ciò è ancora più vero per una Specialità come la Medicina Interna che, oltre a richiedere un anno in più di studi specialistici, necessita di una progressiva acquisizione sul campo di capacità e conoscenze che esige diversi anni di attività se non tutta la vita lavorativa. In questo contesto una insufficiente motivazione rende impossibile raggiungere l'elevata qualità e soddisfazione nel lavoro che è alla base di una vita professionalmente fruttuosa e umanamente appagante. 


\section{Scegli un lavoro che ami...}

Scegli un lavoro che ami, e non dovrai lavorare neppure un giorno in vita tua.

Confucio

In effetti la giornata lavorativa occupa gran parte della nostra vita e la componente economica è solo un aspetto tra quelli che determinano la soddisfazione personale. I bisogni fondamentali degli esseri umani secondo Abraham H. Maslow² (teoria dei bisogni), in ordine di importanza sono: bisogni fisiologici, di sicurezza, di appartenenza, di riconoscimento e autorealizzazione.

Tre sono le principali teorie che hanno preso in considerazione come incrementare la motivazione al lavoro:

1. Teoria dell'equità di J.S. Adams: ${ }^{3}$ la motivazione al lavoro si basa sul modo in cui il dipendente valuta il compito che svolge, sul compenso che ottiene in cambio e sul confronto di questo con quanto ricevuto dal resto dei lavoratori.

2. Teoria della motivazione e dell'igiene di Frederick Herzberg: è possibile identificare i fattori che rendono un lavoro soddisfacente per una persona. Fattori igienici che pur non motivando producono minore produttività e malessere se non soddisfatti (condizioni di lavoro, salario, relazioni interpersonali, stabilità economica, supervisione da parte dei superiori, sicurezza nel lavoro, ecc.). Fattori motivanti che appagano il lavoratore e ne migliorano l'efficienza ma scarsamente influenti se non soddisfatti (promozioni, riconoscimenti, responsabilità, realizzazione personale, posizione sociale, crescita professionale, ecc.)

3. Teoria dei bisogni appresi di David C. McClelland: ${ }^{4,5}$ diverse sono le esigenze che devono essere soddisfatte perché un lavoratore si senta motivato (il bisogno di ottenere risultati, l'equilibrio tra potere e riconoscimento, tra sfida e successo, la necessità di appartenere al gruppo e di avere un contatto con gli altri, ecc.). Si individuano tre fattori motivazionali presenti in ogni essere umano: successo, affiliazione e potere. Ciò che motiva di più una persona dipende dal fattore predominante. Qualunque metodo di analisi si voglia applicare è comunque essenziale rilevare quali sono i fattori che favoriscono la soddisfazione e quali sono quelli che ostacolano un'adeguata motivazione dei lavoratori. FADOI (Federazione Associazioni Dirigenti Ospedalieri Italiani) ha coinvolto i Soci in una survey con l'obiettivo di acquisire dati sulla realtà lavorativa nei reparti di Medicina Interna e di mettere a fuoco, tra l'altro, anche i fattori più soddisfacenti e quelli meno gratificanti dell'attività lavorativa.

Sono state proposte tre schede separate volte ad in- dividuare: A) gli indicatori di qualità del lavoro svolto, B) gli aspetti più soddisfacenti e più importanti dell'attività di reparto, nonché gli aspetti peculiari e da modificare, C) la qualità dei rapporti tra i Medici Internisti e le altre Unità Operative dell'Ospedale.

\section{Attività lavorativa nei reparti di medicina interna}

Uno degli aspetti più importanti che caratterizzano i reparti di Medicina Interna, che li differenzia da quelli di altre specialità, è la grande eterogeneità delle attività svolte, influenzata da numerose variabili: grandezza dell'ospedale, collocazione dell'ospedale in grande, media o piccola città, altre specialità presenti nella struttura, presenza o meno di Pronto Soccorso, numero e competenza dei medici a disposizione, tipologia della struttura (azienda ospedaliera, azienda universitaria, presidio ospedaliero, ospedale classificato, struttura convenzionata, IRCCS, ecc.), strumentazione a disposizione, legislazioni e indirizzi differenti delle regioni. Si può dire che ogni Unità Operativa di Medicina Interna ha una propria caratteristica attività. Tutto ciò rende estremamente difficile trovare gli elementi comuni che caratterizzano la specialità. Peraltro una serie di attività vengono svolte in tutti i reparti di Medicina Interna, per esempio: i) il giro visita; ii) la cartella clinica; iii) l'attività burocratica e gli impegni amministrativi; iv) il colloquio con i pazienti ed i familiari; v) la dimissione del paziente; vi) la gestione del fine vita e del dolore; vii) le consulenze; viii) i turni di guardia e di reparto; ix) l'aggiornamento e la formazione; $\mathrm{x}$ ) le riunioni, i meeting e il lavoro in equipe; xi) l'utilizzo di strumentazioni; xii) gli ambulatori.

I pazienti ricoverati nei reparti internistici hanno sempre avuto alcune caratteristiche, sia per il difficile inquadramento clinico-terapeutico sia per la necessità della presa in carico continuativa, tali da richiedere l'apporto contemporaneo di più medici. La parte centrale dell'attività è sempre stata la cosiddetta visita medica, momento fondamentale effettuato al letto del paziente dall'equipe medica dove elaborare e discutere quotidianamente l'iter diagnostico-terapeutico dopo verifica delle condizioni cliniche e la valutazione delle indagini effettuate. Tale attività impegnava la maggior parte del tempo lavorativo nelle ore antimeridiane. I cambiamenti epidemiologici e demografici, la riduzione del personale disponibile, l'aumento del tempo necessario ad altre attività, la progressiva riduzione del peso dell'esame clinico a vantaggio degli esami diagnostici, l'informatizzazione del lavoro, il progresso nelle specifiche attività di varie figure professionali, hanno messo in crisi l'organizzazione della visita medica. In primo luogo è evidente che la complessità dei pazienti e la complessità delle attività da svolgere sul malato hanno necessariamente cambiato 
il significato della visita medica modificandone anche il nome in giro visita. Tale attività non può più essere ad esclusivo appannaggio dei medici ma necessita dell'apporto attivo di tutte le altre figure professionali che intervengono sul paziente. È da molti anni che è richiesta la presenza della professionalità infermieristica (un tempo rappresentata dal Caposala) fondamentale per integrare e omogeneizzare le attività e per garantire la trasmissione delle informazioni sull'andamento del paziente. Attualmente ogni ricoverato avrebbe diritto alla definizione di un Piano Assistenziale Individuale quotidiano (PAIq) a cui devono contribuire tutte le figure professionali via via interessate a quel determinato paziente a seconda delle necessità (medici internisti, medici specialisti d'organo, infermieri, riabilitatori, nutrizionisti, assistenti sociali, ecc.) con integrazione delle attività. Nella frenetica attività quotidiana e nelle enormi problematiche gestionali siamo molto lontani da una tale organizzazione. Nel tempo le varie figure si sono evolute singolarmente e sempre più agiscono separatamente nella gestione e cura del paziente. Non stupisce che in tale situazione nella survey FADOI la visita collegiale ha ottenuto un punteggio basso tanto nelle attività più soddisfacenti che in quelle più importanti e peculiari. È dunque necessario un profondo ripensamento di tale attività. I momenti di discussione e di approfondimento devono essere necessariamente trovati al di fuori del giro visita in modo da impegnare il gruppo di operatori sanitari per un tempo limitato. Sono state, pertanto, ipotizzate riunioni di valutazione da effettuare al mattino e successive riunioni di verifica a fine giornata lontano dal letto del paziente (briefing e debriefing). In questo modo i vari operatori possono in teoria effettuare le proprie attività separatamente ed indipendentemente sul paziente con i tempi ed i modi a loro più consoni senza rinunciare al confronto e alla multidisciplinarità. La convulsa attività della corsia, la scarsa dotazione di personale, la diversa disponibilità di tempo dei vari operatori, l'enorme mole di incombenze burocratiche, la concentrazione delle varie attività dell'ospedale al mattino spesso non consentono la contemporanea presenza delle varie figure professionali, facendo perdere il momento di confronto. Ne deriva una importante sensazione di solitudine del medico che deve effettuare il giro visita molte volte senza nemmeno l'ausilio ed il contraddittorio con i colleghi e gli infermieri. Questa situazione diviene poi problematica se si associa alla scarsità di risorse che, insieme alla necessaria turnazione nelle 24 ore, non consente all'operatore la continuità di presa in carico del paziente per diversi giorni consecutivi: il paziente internistico richiede talora molto tempo per essere completamente inquadrato. È una esigenza molto sentita dai medici tanto che nella survey hanno avuto un punteggio molto elevato la presenza in visita dello stesso medico per al- meno 5 giorni consecutivi alla settimana come indicatore della qualità dell'assistenza e la continuità assistenziale tra le attività di reparto più importanti. Il risultato è una profonda crisi delle usuali modalità di lavoro che arriva sino a mettere in discussione l'utilità del giro visita nel suo complesso, crisi rafforzata dalla spinta di autonomia delle varie professioni sanitarie che spesso sono state considerate subalterne e di complemento alla figura medica senza riconoscerne la professionalità e l'indipendenza. Sicuramente è necessario un ripensamento delle modalità di lavoro che consenta la multisciplinarità nella gestione dei pazienti internistici così complessi e spesso fragili.

Uno dei sostanziali cambiamenti che si sono progressivamente imposti nell'ambito della professione medica e, in particolare, nell'attività ospedaliera è stato l'enorme incremento del tempo da dedicare alle incombenze burocratiche in generale e segnatamente alla gestione della cartella clinica. Gli item della survey FADOI non hanno esplorato questo aspetto in quanto ormai evidente a tutti. Forse non è stato chiarito a sufficienza nelle apposite sedi che il tempo necessario agli aspetti burocratici viene sottratto al tempo di cura del paziente, al tempo di colloquio con il paziente ed i familiari e al tempo per l'aggiornamento professionale. Di fatto è una sottrazione di risorse di personale che va ulteriormente ad acuire la grave carenza di medici ed infermieri diffusa in tutta Italia, soprattutto nelle regioni in piano di rientro, legata al ripetuto sottofinanziamento della Sanità. Ciò si associa alla sempre crescente richiesta alle strutture ospedaliere, prevalentemente ai Pronto Soccorso e alle Medicine Interne, di presa in carico di problematiche squisitamente sociali che con il cambiamento della struttura delle famiglie (composte da una sola persona $31,9 \%$, coppie senza figli $20,5 \%$, con un solo adulto $10 \%),{ }^{6}$ con l'invecchiamento della popolazione, con l'incremento dell'attesa di vita dei pazienti con gravi malattie e disabilità insieme al prolungamento della vita lavorativa per il procrastinarsi della pensione (mancata possibilità di accudire i familiari) non riescono ad essere gestite nelle famiglie e dal territorio. Nelle risposte dei medici FADOI ai questionari si evidenziano nel gruppo degli indicatori di qualità il rapporto tra numero di medici e il numero di infermieri con il numero pazienti in carico, tra le attività più importanti il tempo da dedicare all'assistenza e tra le attività da modificare la dotazione organica e l'eccesso di pazienti in carico avendo tutti raggiunto i punteggi più alti. È evidente come i medici sentano la necessità di dedicare più tempo alla cura diretta del paziente $o$ incrementando la pianta organica o delegando ad altre figure parte delle incombenze burocratiche e sociali, recuperando quel rapporto medico-paziente che è l'attività ritenuta più soddisfacente e utile.

Attualmente è ineludibile l'obbligo di garantire 
l'autodeterminazione del paziente tramite il consenso informato, che da orale è divenuto scritto e da attività utilizzata solo per le decisioni dubbie e rilevanti (in quanto le altre erano ricomprese nel rapporto di fiducia medico-paziente) ormai è necessario per ogni atto o decisione. Il problema è come illustrare ai pazienti ed ai familiari, in modo chiaro, comprensibile ed efficace situazioni sempre più complesse, che possono prevedere attività spesso ai limiti dell'accanimento terapeutico ovvero l'impiego di terapie o presidi con elevati rischi di effetti avversi. Gli utenti sono oggi molto più informati ma spesso in modo confuso o errato. Nello stesso tempo la continua delegittimazione della figura del medico da parte degli organi di stampa e dei social, ha favorito un clima di sospetto, sfiducia e diffidenza nella popolazione che si avvicina agli operatori sanitari. È presente anche la necessità di educare il paziente ed i familiari/caregiver alla prevenzione, all'uso di dispositivi terapeutici, al monitoraggio delle terapie, alla corretta assunzione dei farmaci, alla corretta alimentazione, all'attività fisica, ecc.. Tutto ciò ha messo in luce la grave carenza di formazione del medico sulle modalità di comunicazione, argomento praticamente inesistente nel corso degli studi. Il tema è particolarmente sentito dagli internisti che lo reputano una delle caratteristiche dello specialista in Medicina Interna in quanto la comunicazione con pazienti e familiari in situazioni complesse o in polipatologie in stadi avanzati o addirittura nel fine vita sono spesso delegate dai vari specialisti d'organo all'internista. Inoltre una buona capacità comunicativa è il primo presidio per arginare il dilagante aumento dei contenziosi, delle aggressioni agli operatori sanitari e delle richieste di risarcimento di supposti danni. La comunicazione efficace diviene poi fondamentale nel momento più delicato del ricovero di un paziente, cioè la dimissione. È il momento in cui si deve stilare il Piano Assistenziale Individuale, piano che dovrebbe anche esso essere frutto di una attività multidisciplinare. I pazienti internistici hanno sempre la necessità di proseguire le terapie dopo il ricovero, spesso richiedono controlli e ulteriori indagini, devono essere in grado di adoperare nuovi presidi il cui utilizzo è iniziato durante il ricovero. Questo momento critico necessita di una quantità di tempo che attualmente non è a disposizione degli operatori sanitari. La dimissione che deve essere preparata con adeguato anticipo, utile per affrontare con paziente e familiari le varie necessità, richiederebbe anche una stretta integrazione con il territorio, soprattutto con il medico curante, figura completamente assente nel percorso ospedaliero. Tutte le Medicine Interne prevedono la presenza continuativa di un medico anche nelle ore pomeridiane e notturne sia per i pazienti ricoverati nei propri reparti sia per eventuali consulenze o interventi urgenti nell'intero ospedale. Questa attività richiede ovviamente una tur- nistica che nell'attuale situazione di carenza di personale complica ulteriormente le problematiche prima riportate. Riduce il tempo dedicato da ciascun medico all'iter diagnostico-terapeutico dei pazienti e soprattutto frammenta l'assistenza rendendola discontinua. Si comprende come nelle risposte dei medici all'indagine abbia ricevuto un valore molto elevato tra le cose da modificare la organizzazione del lavoro e dei turni.

A dispetto di ciò, la definizione della Medicina Interna rimane, comunque, complessa risultando difficile l'individuazione del suo ambito di applicazione, specie dopo che la maggiore rilevanza clinica della strumentazione ed il progressivo perfezionamento tecnologico ha dato il via libera alla formazione delle discipline specialistiche che si sono, progressivamente, ritagliate la propria autonomia indebolendo la funzione dell'Internista. ${ }^{7}$ Infatti, man mano che l'assistenza sanitaria si è specializzata, anche le aspettative dei pazienti di essere gestiti da uno specialista sono andate crescendo. La ricerca biotecnologica ha assunto un'importanza sempre maggiore nelle diverse branche medico-chirurgiche, contribuendo già ora, e sicuramente ancor più nel prossimo futuro, a progressi in ambito diagnostico e terapeutico fino a poco tempo fa largamente inimmaginabili.

Le formazioni specialistiche gemmate dalla Medicina Interna e quelle parti di esse che si sono ulteriormente settorializzate hanno accolto la ricerca scientifica e l'innovazione tecnologica utilizzandole nella pratica clinica per migliorare le capacità diagnostico-terapeutiche e per soddisfare la domanda di salute, acquisendo visibilità nell'immaginario collettivo e agli occhi dei decisori politici che hanno visto in esse il concretizzarsi della speranza di una vita in buona salute. Relativamente all'applicazione dell'innovazione tecnologica a supporto dell'attività clinico-assistenziale ospedaliera gli internisti non hanno investito a sufficienza per sfruttarne le opportunità e per rimuovere le principali barriere che ne ostacolano o bloccano l'adozione e la diffusione. Questo procedere tumultuoso della tecnologia ha fortemente condizionato le politiche economiche che hanno privilegiato gli investimenti, nelle organizzazioni aziendali, a favore delle sub-specialità, sottoponendo la Medicina Interna ad una elevata pressione esterna che ha generato disparità nei rapporti con le altre specialità. Così, i posti letto nelle Medicine sono stati ridotti ma i pazienti continuano ad ammalarsi ugualmente e sono colpiti da patologie complesse che interessano più organi ed apparati, motivo per cui non hanno accesso nei reparti specialistici. I posti letto nelle Medicine scarseggiano e allora si aggiungono i letti, ora un terzo letto in una stanza da due, ora fuori reparto, magari in una struttura lontana con scarsa possibilità di controllo e monitoraggio da parte di chi lo ha in carico. Tutto ciò ha, di fatto, generato una situazione di stress cro- 
nico dell'assetto sanitario nel suo complesso, radicato nell'ambiente di lavoro e nella cultura organizzativa. Il burnout organizzativo ha contaminato gli Internisti che hanno cominciato a percepire esaurimento emotivo, depersonalizzazione e ridotta sensazione di realizzazione personale con potenziali conseguenze negative per i medici stessi e per gli esiti delle cure. ${ }^{8-}$ ${ }^{10}$ L'aspetto più eclatante di questa situazione emozionalmente negativa è stata la rinuncia alla cura della persona e la delega ad altri specialisti con conseguente indebolimento della figura e ruolo dell'internista.

\section{Aspetti da modificare}

Uno dei dati che si evidenziano nelle unità di Medicina Interna, comune anche ad altre specialità, è l'elevata età media dei medici che vi opera. Nella survey di FADOI solo il 5,8\% dei questionari è stato compilato da medici di età inferiore ai 30 anni ed il 33,9\% tra 30 e 45 anni, ben il 32\% ha un'età superiore ai 56 anni. In queste condizioni ci troveremo presto in carenza di medici e nella difficoltà di formarli, poiché una specialità che richiede un così lungo tempo di apprendimento, necessita di un continuo inserimento di giovani che possano iniziare l'attività sotto il tutoraggio dei più esperti. È peculiare, anche in confronto con gli altri reparti ospedalieri, il fatto che oltre il $40 \%$ dei medici che operano nelle medicine non hanno la specializzazione in Medicina Interna (dato della survey). Numerosi sono gli strutturati con specializzazioni equipollenti o affini e molti hanno più di una specializzazione. Purtroppo, in parte, questa situazione si va a inquadrare in una visione riduttiva della Medicina Interna che è stata considerata per anni non una specializzazione ma un tipo di reparto dove dovevano essere trattati i pazienti di non stretta pertinenza specialistica. Una corsia reputata meno impegnativa, con competenza globale ma generica, a bassa assistenza con prevalente gestione di malati cronici. Non a caso molti reparti sono ancora denominati Medicina Generale, termine che crea tra l'altro confusione nella popolazione, ma anche nelle istituzioni, con l'attività dei Medici di Medicina Generale del territorio. Nello stesso tempo la presenza di specialisti con approfondita competenza in particolari sub-discipline mediche ha incrementato la capacità di diagnosi e cura stimolando l'approfondimento di particolari situazioni, migliorando l'appropriatezza nel trattamento di diverse malattie. Peraltro molti degli specialisti d'organo più anziani hanno frequentato per molti anni le corsie di Medicina Interna prima di essere assunti o di specializzarsi, acquisendo sul campo il metodo internistico (sino a circa 25 anni fa non era necessaria la specializzazione nella disciplina per essere assunti). Il metodo internistico è stato considerato tra le attività distintive più importanti che caratterizzano l'attività del reparto da chi ha risposto alla survey. In pratica chi lavora nelle corsie di Medicina Interna è cosciente dell'esistenza di un approccio peculiare dell'internista alla gestione dei malati. Probabilmente non tutti intendono la stessa cosa ma alcune caratteristiche sono comunemente evidenziate: l'inquadramento del paziente nella sua globalità, la presa in carico del paziente e non della malattia o del singolo organo, la particolare attenzione alle interazioni ed agli effetti avversi delle terapie, la necessità di individuare una scala delle priorità da affrontare per ogni singolo paziente, la necessità di trattare le malattie acute tenendo conto delle numerose patologie croniche coesistenti, dell'età e dello stato degli emuntori, la necessità di operare in più medici per arrivare a definire le diagnosi difficili, la capacità di intervenire come un direttore d'orchestra per armonizzare gli interventi dei vari operatori sanitari e dei vari specialisti, la capacità di adattare le linee guida, prevalentemente d'organo o di singola malattia, alle necessità di un paziente con diverse malattie, la rilevanza data all'anamnesi e all'esame obiettivo (spesso di minore interesse per gli specialisti d'organo che si basano prevalentemente sulle indagini) nell'inquadramento e nella cura del paziente, l'attenzione prestata alla comunicazione con i pazienti e familiari, ecc.. Nello stesso tempo nelle nostre corsie si è andata progressivamente perdendo la necessità di effettuare in prima persona procedure diagnostiche e di utilizzare apparecchiature diagnostico-terapeutiche che vengono delegate ad altre figure professionali o ad altri specialisti. Tali attività vengono così a perdere il contributo, all'indagine, della parte clinica divenendo attività tecniche standardizzate. In alcuni ospedali la toracentesi è effettuata dal chirurgo toracico, la ventilazione non invasiva (NIV) dall'anestesista o dallo pneumologo, l'ecografia dal radiologo, persino il posizionamento di sondini naso-gastrici possono essere ad appannaggio esclusivo di figure professionali. La richiesta di modificare questa situazione sia per migliorare la qualità sia per ridurre i tempi di attesa è molto forte soprattutto tra i giovani internisti. Molto sentita è la necessità di acquisire competenze in ecografia e nella gestione della NIV. L'impegno delle Società Scientifiche e delle Scuole di Specializzazione in tal senso cerca di colmare il deficit formativo ma le amministrazioni si oppongono non fornendogli gli spazi e le apparecchiature necessarie.

Come è stato già riportato i medici internisti hanno individuato tra le situazioni da modificare soprattutto quelle che non consentono di seguire continuativamente i pazienti per il tempo necessario: scarsità della dotazione organica, eccesso di pazienti in carico, organizzazione del lavoro e dei turni, presenza di pazienti in altri reparti (appoggi). È questa una situazione di disagio in quanto gli internisti sentono di non dare ai pazienti quanto dovrebbero in termini di attività clinica. 
Non è raro che alcuni colleghi preferiscano limitare la propria attività ai turni pomeridiani o notturni evitando la presa in carico del paziente, in pratica rinunciando alla parte clinica. Manca il tempo per l'approfondimento del singolo caso, per la discussione con i colleghi di altre specialità e per l'aggiornamento. Il problema è prevalentemente individuato nell'eccesso di burocrazia e nella discontinuità nell'assistenza al paziente. Probabilmente il concetto di burocrazia deve essere inteso in modo estensivo comprendendo sia le infinite disposizioni delle singole aziende sia le procedure imposte dalle regioni e dal ministero sia la gestione della cartella clinica sia le regole per la privacy, per la trasparenza, per il consenso informato, per la prescrizione dei farmaci, ecc. La grande aspettativa di semplificazione riposta nella progressiva informatizzazione delle attività si è rivelata vana. Le incombenze si sono ulteriormente incrementate e il computer ha sottratto ulteriormente tempo al rapporto medico-paziente. Certo la quantità di dati a disposizione e la possibilità di utilizzarli sono enormemente incrementate ma la richiesta ai medici di inserimento di tali dati si è moltiplicata soprattutto a fini amministrativi ed epidemiologici. La possibilità di effettuare indagini e di utilizzare la parte informatica si scontra inoltre con la carenza e l'obsolescenza delle apparecchiature a disposizione. Puntualmente nella survey ottiene un punteggio elevato tra le attività da modificare la tecnologia non adeguata. Infine è evidente che la modalità di collaborazione tra i medici dello stesso reparto e con quelli di altre specialità deve essere modificata. Nelle varie equipe si vive la difficoltà di formare un gruppo omogeneo di "formare una squadra". Sembra che ognuno pratichi una sua propria attività medica con obiettivi simili ma non identici. Non esiste più una graduazione delle competenze e delle responsabilità così come una progressione della carriera lavorativa. Le responsabilità dei direttori di struttura, delle amministrazioni e della legislazione è evidente, sempre più impegnati al raggiungimento di obiettivi economici ed epidemiologici trascurando gli aspetti qualitativi dell'assistenza e la motivazione degli operatori. Nelle attività mediche è molto rilevante la medicina difensiva che complica ulteriormente i rapporti all'interno dell'equipe e con i consulenti di altre specialità. Si deve recuperare il piacere di lavorare fianco a fianco per garantire la migliore assistenza possibile ai pazienti con un percorso di crescita lavorativa quotidiano imparando gli uni dagli altri.

\section{Aspetti gratificanti}

Negli ultimi anni la figura di quello che una volta era definito il clinico medico è tornata, appropriatamente, a svolgere un ruolo di grande prestigio negli ospedali come documentato da molta letteratura. Infatti, la progressione delle capacità terapeutiche della medicina specialistica nella cura delle malattie acute, il miglioramento delle condizioni sociosanitarie e l'aumento della sopravvivenza hanno portato progressivamente a una profonda modificazione degli scenari di cura in quanto, parallelamente all'invecchiamento della popolazione, si è avuto un notevole incremento delle malattie ad andamento cronico, spesso presenti contemporaneamente nello stesso individuo.

Tutto ciò ha creato problematiche inedite con le quali il Sistema Sanitario deve confrontarsi e che sono costituite da longevità, fragilità, disabilità, polipatologia, cronicizzazione, interconnessioni morbose capaci di condizionare l'evolutività clinica e le complicanze. L'applicazione di tecnologie diagnostiche e di presidi terapeutici sempre più sofisticati e l'esito non più necessariamente funesto di molte malattie acute hanno cambiato il contesto epidemiologico delle malattie nel quale, sia pure in presenza di uno sbilanciamento dell'attuale disponibilità dell'innovazione tecnologica a vantaggio delle sub-specialità, la figura dell'internista è oggi al centro dell'attenzione della comunità scientifica, della Società in genere e dei decisori politici, in quanto ha dimostrato di possedere quel background metodologico, culturale e professionale che rende possibile la gestione globale di situazioni cliniche complesse, elevandosi ben oltre ogni forma di modernizzazione dei sistemi diagnostici e terapeutici spesso mal controllabili dagli stessi utilizzatori. ${ }^{11} \mathrm{Nel}$ contesto del rinnovato interesse per il ruolo dell'internista vengono evidenziati nelle risposte alla survey gli aspetti ritenuti peculiari, come già riportato precedentemente: visione globale, comunicazione con pazienti/familiari, valutazione delle priorità terapeutiche, metodo internistico, valutazione delle priorità diagnostiche, management delle comorbilità, diagnosi difficili. Questa coscienza di una forte identità della specialità si concretizza nell'individuazione di molti aspetti soddisfacenti e motivanti nell'attività quotidiana. In primo luogo l'internista continua a trarre enorme soddisfazione dal rapporto con il paziente che rappresenta la centralità e la peculiarità in generale dell'attività medica ma insostituibile nell'ambito della Medicina Interna. Il rapporto è divenuto nel tempo sempre più complesso ed impegnativo con l'accrescimento del ruolo del paziente nell'iter decisionale ma ha portato con se anche la necessità di istaurare legami profondi con il malato che rendono più completa $\mathrm{e}$ soddisfacente l'attività del medico stesso. Certo gli aspetti legati al consenso informato e alla Legge 219 del 22 dicembre 2017 (biotestamento) continuano a richiedere riflessioni e approfondimenti proprio per trovare un equilibrio tra la necessità di informazione, l'autodeterminazione del paziente e il ruolo del medico evitando di minare proprio quel rapporto di fiducia che è alla base di ogni atto medico. Il principale obiettivo è sempre il bene del paziente e l'internista 
trae enorme soddisfazione nel risolvere o migliorare le problematiche del malato tanto più quanto più sono complesse e difficili. È uno degli aspetti più soddisfacenti individuati nella survey. In confronto con altre sub-specialità dove è preponderante l'individualità e spesso la competitività, nella Medicina Interna prevale la necessità di lavorare in gruppo. Necessità che arricchisce professionalmente e umanamente permettendo di risolvere le problematiche più complesse. Non stupisce che il confronto quotidiano con i colleghi, i momenti di incontro e discussione e la collaborazione con tutti gli operatori sanitari siano annoverati tra gi aspetti più soddisfacenti della professione. Peraltro, tale confronto, sembra sia auspicato al di fuori della visita collegiale in momenti dedicati senza la presenza del paziente. Ciò in parte contrasta con la sempre crescente necessità di coinvolgere il malato nel processo decisionale e con l'opportunità di valutare ogni provvedimento alla luce delle condizioni cliniche del paziente. Infine c'è una grande necessità di riappropriarsi in prima persona dell'esecuzione di indagini strumentali e di procedure diagnostico-terapeutiche, in parte per rendersi autonomi nel percorso diagnostico-terapeutico, in parte per la coscienza che ogni indagine ha maggiore efficacia se viene eseguita direttamente dal clinico che deve inquadrare il paziente. Ormai c'è la coscienza che il supporto ecografico è parte integrante dell'esame obiettivo del malato e che molti presidi tecnici sono necessari nelle corsie di Medicina Interna che ricoverano abitualmente pazienti critici.

\section{Gli ambiti del miglioramento}

Dalla crisi d'identità degli anni ' 90 e dal successivo recupero della centralità dell'internista piuttosto involontario, non controllato e non ottenuto attraverso una strategia, la Comunità degli Internisti deve trarre l'insegnamento che è necessario, investire energie e risorse nel progresso tecnologico per gli indubbi vantaggi che ne possono derivare in ambito epidemiologico, di ricerca clinica, di analisi delle immagini (US, TC, MRI, PET), di dati relativi al funzionamento di organi ed apparati (EEG, ECG, Saturimetria, Pletismografia), di segnali per la valutazione della funzionalità di apparati biologici, di discipline biomolecolari e di modellistica per analisi di sistemi e sottosistemi. Nell'ottica sopra delineata è importante che la Comunità degli Internisti sia aperta ad accogliere nuovi sistemi assistenziali, impegnandosi nel loro sviluppo avendo così la possibilità di modellarli in modo sartoriale, rispettando le variegate realtà sanitarie del nostro paese, considerando che una delle caratteristiche comuni è il ruolo fondamentale delle Aree Mediche che ospitano nei propri reparti il $13-15 \%$ di tutti i ricoveri ospedalieri, provenienti per 1'87\% dal Dipartimento Emergenza Urgenza (DEA) e costituiti da pazienti per il $60 \%$ con età superiore ai 75 anni, molto spesso instabili o critici.

Nella necessità di ripensare i modelli organizzativi per renderli quanto più funzionali possibili e porli nella condizione di far fronte ad almeno due dei cambiamenti che si sono venuti profilando con forza crescente in questi ultimi anni: i) l'invecchiamento della popolazione, che ha comportato un aumento negli ospedali di pazienti anziani, nella stragrande maggioranza dei casi, comorbidi, complessi e fragili; ii) lo sviluppo tecnologico, che ha cambiato in maniera significativa le modalità di approccio a numerose patologie acute, generando esiti molto favorevoli ma favorendo la cronicizzazione.

Potrebbe essere opportuna la creazione di un'Area Medica Omogenea affiancata dall'azione di una istituzione che potremmo definire Centrale di Continuità Ospedale-Territorio in grado, attraverso il rapido snellimento delle procedure burocratiche, di contribuire, grazie ad una rapida collocazione dei pazienti clinicamente stabilizzati in strutture o organizzazioni territoriali, ad un adeguato turnover dei pazienti ricoverati nelle corsie per acuti, spesso rallentato dalle così dette dimissioni difficili. Per dimissione difficile intendiamo la dimissione che, nel rispetto della continuità terapeutica ed assistenziale, necessita di un consumo di risorse economiche, umane ed organizzative che vanno oltre la potenzialità del paziente e dei suoi familiari implicando un coinvolgimento particolare di quei presidi territoriali comprendenti i medici di famiglia, i servizi infermieristici ed assistenziali domiciliari, le lungodegenze extraospedaliere, le residenze sanitarie assistenziali, le case protette e le case di riposo. La dimissione difficile si verifica quando ad un evento acuto, fa seguito una situazione di disabilità permanente o temporanea, che richiede una riorganizzazione familiare già resa precaria dai cambiamenti della situazione finanziaria che hanno limitato le risorse disponibili e dalle mutate caratteristiche sociali della popolazione.

Vanno individuate le discipline omogenee per la macro-aggregazione nell'Area Medica senza abolire l'articolazione in Unità Operative. Il modello organizzativo che potrebbe rispondere in modo più aderente a tali nuove esigenze è quello definito per intensità di cure. ${ }^{12}$

Tale modello consente di: i) collocare il paziente in relazione alla sua criticità e severità e non semplicemente per patologia; ii) evitare di rendere frammentaria la collocazione strutturale logistica dei pazienti; iii) considerare il paziente nella sua interezza, complessità e polipatologia; iv) prevedere approcci diagnostico-terapeutici integrati anche attraverso l'utilizzo di supporti tecnologici appropriati alla situazione di ogni singolo paziente.

Tale modello può essere applicato, con la massima 
flessibilità, in ambito dipartimentale attraverso la collocazione in un'area con caratteristiche strutturali che ne possano consentire l'attuazione.

È necessario costruire un assetto organizzativo strutturato per intensità di cure in cui sia prevista: i) Un'area di degenza ad alta intensità di cure, per pazienti che non necessitano di accesso in U.T.I., la cui appropriatezza di ricovero sia valutata in base al NEWS. Attrezzata con monitoraggio multiparametrico wireless, con spazi adeguati per consentire la NIV, con pompe idonee per infusioni parenterali ed enterali e dotata di un sistema informatico in rete. La strutturazione logistica deve poter consentire un'assistenza infermieristica ad alta intensità, rapidamente attivabile in caso di necessità e la presenza medica deve differenziarsi per un maggior numero di accessi programmati ed opportunamente documentati; ii) Un'area di degenza per pazienti con fase di malattia in acuzie ma con minor grado di instabilità e criticità; iii) Un'area di degenza a più bassa intensità di cure dove allocare il paziente sufficientemente stabilizzato ma ancora a rischio di ricadute o complicanze e che, comunque, necessita ancora di terapia complessa. Ovvero in attesa della presa in carico da parte del territorio.

La creazione delle aree deve consentire la collocazione e passaggio dei pazienti da un livello ad un altro a seconda dei bisogni. Il personale da dedicare alle aree di cui ai punti b. e c. è quello ordinario e fornirà ai pazienti un'assistenza strutturata o a richiesta provvedendo alle necessità assistenziali: mobilizzazione, cura della persona, alimentazione, igiene, terapia, ecc.. Tutte queste aree devono avere una sistemazione adeguata in spazi opportunamente collegati e adiacenti.

Questo modello, che non comprende le Unità di Terapia Intensiva, deve prevedere percorsi interni che possano consentire di accogliere in sicurezza pazienti ancora instabili provenienti anche dalle U.T.I. e pazienti chirurgici con patologie mediche complesse e instabili nell'immediato post-operatorio.

Deve quindi esistere ed essere strutturata un'interfaccia tra Pronto Soccorso e reparti di degenza per attuare, fin dall'accettazione, gli snodi decisionali di tipo diagnostico-terapeutico, stratificando la gravità del paziente e definendo criteri condivisi di allocazione all'interno delle aree a diversa intensità. Le responsabilità clinico-gestionali per ogni singolo paziente sono in carico all'Unità Operativa alla quale il paziente è assegnato. L'organizzazione del lavoro deve essere adeguatamente regolamentata e strutturata con un coordinamento che fa capo al Consiglio di Area Medica (Direttori di UOC, UOSD e UOS) con un Direttore di Area Medica cui compete la gestione degli indicatori di esito, i rapporti con il Bed Management e la Direzione Sanitaria di Presidio e la interazione con la Direzione Aziendale.

\section{Conclusioni}

Nel corso della millenaria storia della medicina, lo scopo del medico è sempre stato, e rimane, quello di diagnosticare il problema, adottare quei provvedimenti terapeutici in grado di ripristinare l'equilibrio del paziente e ottenerne la guarigione. ${ }^{13} \mathrm{Nel}$ corso di questa lunga storia, la pratica della medicina è passata dall'approccio olistico a quello delle spiegazioni scientificamente moderne e supportate dai progressi tecnologici. ${ }^{14}$ Quest'approccio ha fatto temere a molti lo smarrimento dell'universalità sia per il paziente che per il medico. ${ }^{15}$ Capire quali pressioni esterne abbiano portato al cambiamento del paradigma della medicina è fondamentale per comprendere il significato del cambiamento dell'incontro fra medico e paziente nel XXI secolo. È questo il contesto in cui si inserisce il concetto della insostituibilità della Medicina Interna perché alla base c'è la buona formazione dell'internista che acquisisce la struttura culturale, metodologica e professionale che gli consente di affrontare la complessità del paziente anche nello scenario attuale dominato da profonde trasformazioni sociali, dall'adozione delle innovazioni tecnologiche, dal cambiamento dell'epidemiologia delle malattie e dal prorompente ingresso nella pratica clinica della medicina basata sull'evidenza derivata da prove sperimentali su vaste popolazioni di soggetti. Non è alla semplicistica adozione delle soluzioni tecnologiche che la classe medica deve delegare la gestione dell'eterogeneità dell'incontro clinico ma, al contrario, è l'acquisizione della tecnogovernanza che deve guidare la gestione della malattia nella cornice delle linee guida e delle raccomandazioni di buona pratica clinica. ${ }^{16-18}$ Questo aspetto rappresenta il vero punto di valenza dell'internista in quanto ne mette in luce la forte identità. La rinascita della Medicina Interna è ormai tangibile e noi internisti non dobbiamo perseguire la perfezione ma, al contrario, avviare un processo di miglioramento continuo attraverso il quale superare la resistenza al cambiamento che ci ha contrassegnati nei decenni scorsi oscurando la nostra branca. Ispirandoci a questi principi, nel corso della formazione specialistica, vanno migliorate le nostre competenze per essere pienamente in grado di offrire una prestazione sanitaria di qualità che metta al centro il paziente. Nel percorso formativo dell'internista va inserita la comunicazione che assume un ruolo sempre più centrale nelle sue principali articolazioni: medico-paziente, medico care-giver, medico-medico. Le innovazioni tecnologiche, la complessità degli interventi sanitari, la frammentazione del percorso di cura, anche per cause contingenti, può essere superata solo con un'adeguata e strutturata comunicazione. È proprio nel contesto della comunicazione che si inserisce l'empowerment del paziente, concetto nato intorno al 2000 e che è ufficialmente diventato il nuovo obiettivo della Sanità finalizzato a mettere il paziente al cen- 
tro delle sue cure, renderlo costantemente informato e partecipe nel processo decisionale e di cura, poiché le personali decisioni di un paziente possono influenzare anche la sua stessa condizione di salute psico-fisica. L'empowerment del paziente è di fondamentale importanza soprattutto nei malati cronici. Un dato di fatto di cui dobbiamo avere consapevolezza perché è la nostra forza, è che la Medicina Interna è l'asse portante del Sistema Sanitario tanto che è presente in tutti gli ospedali, dove si fa carico della grande maggioranza dei ricoveri acuti di area medica garantendo continuità nelle 24 ore per 365 giorni all'anno. Per tali ragioni vanno definite le attività prioritarie che, se affrontate con competenza manageriale, possono rendere le nostre scelte compatibili con un Sistema Sanitario Sostenibile e versatile nella forma di assistenza sanitaria messa a disposizione grazie alla poliedrica gamma di expertise acquisita e che deve annoverare anche la NIV, l'ecografia bedside, le procedure invasive, l'impianto dei PICC, la gestione del paziente critico adottando sistemi tecnologici (ad es., il monitoraggio wireless). La cultura della cronicità patrimonio dell'internista è il presupposto per l'impulso all'affermazione definitiva della figura dell'Hospitalist il cui ruolo va visto in una complessa attività strutturata di interfaccia soprattutto con i percorsi chirurgici. Proprio per la profonda conoscenza del malato cronico è sempre più importante il ruolo dell'internista nell'individuazione dei setting assistenziali alternativi al regime di ricovero (Day Hospital, PAC, Ambulatori Specialistici) che possono decongestionare gli ospedali e nell'integrazione con i servizi territoriali tramite la costituzione delle Centrali di Continuità Ospedale Territorio (CCOT) istituzionalmente in relazione con le Case della Salute, le Lungodegenze, gli Hospice, i CAD e con i Servizi Sociali dei Comuni per facilitare le cosiddette dimissioni difficili. Si può concludere con le parole dello scrittore Don De Lillo, tratte dal libro $R u$ more bianco in cui, nello scenario medico-sociale così ampio e complesso in cui è collocata la figura dell'internista, tratteggia una riflessione che bene mette in luce il ruolo centrale dell'internista: A New York.... chiedono se hai un buon internista. Ecco dove sta il potere. Gli organi interni. Fegato, reni, stomaco, intestino, pancreas. La medicina interna è la pozione magica .... Da un buon internista derivano energia e carisma, del tutto al di là della cura che somministra. Si chiedono notizie di fiscalisti, di operatori finanziari, di spacciatori. Ma a contare sul serio sono gli internisti. ${ }^{19}$

\section{Bibliografia}

1. Giannandrea F, Ferraro P. Work Engagement. La ricerca della felicità nei luoghi di lavoro. Edizioni Ferrari Sinibaldi; 2018.

2. Maslow AH. Motivation and Personality. Harper \& Row; 1954.

3. Adams JS. Injustice in social Exchange. Advances in experimental social psychology. New York: Academic Press; 1965.

4. McClelland DC. The Achieving Society. New York: Van Nostrand Reinhold; 1961.

5. McClelland DC. Power: The inner experience. New York: Irvington; 1975.

6. Annuario Statistico Italiano 2018 - Rapporto ISTAT al $1^{\circ}$ gennaio 2018.

7. Dammacco F. La Medicina Interna e l'approccio olistico al paziente tra globalizzazione e tecnologie avanzate. Recenti Prog Med 2012;103:248-55.

8. Medicina della Complessità: la moderna Medicina Interna. Fondazione Paolo Procacci; 2011.

9. Yates SW. Physician Stress and Burnout. Am J Med 2020;133:2.

10. Lemaire JB, Ewashina D, Polachek AJ, et al. Understanding how patients perceive physician wellness and its links to patient care: A qualitative study PLoS One 2018 https://doi.org/10.1371/journal.pone.0196888

11. Panagioti M, Panagopoulou E, Bower P, et al. Controlled interventions to Reduce Burnout in Physicians. A Systematic Review and Meta-analysis. JAMA Intern Med 2017;177:195-205.

12. Gensini GF, Conti AA, Lippi D. La Medicina Interna: storia, attualità, prospettive. Recenti Prog Med 2005;96:190-3.

13. Landini G. La Medicina interna fra intensità di cure e territorio. Toscana Medica 2017;10.

14. Zaharias G. What is narrative-based medicine? Narrative-based medicine. Canad Fam Phys 2018;64

15. Dammacco F. La Medicina Interna e l'approccio olistico al paziente tra globalizzazione e tecnologie avanzate. Recenti Prog Med 2012;103:248-55.

16. Wolf A, Moore L, Lydahl D, et al. The realities of partnership in person-centred care: a qualitative interview study with patients and professionals. BMJ Open 2017; 7:e016491.

17. May C. Technogovernance: Evidence, subjectivity, and the clinical encounter in primary care medicine. Social Sci Med 2006;62:1022-30.

18. Lenti S, Felici M, Campanini M, et al. La comunicazione con il paziente e tra i professionisti nella gestione del paziente complesso in Medicina Interna. Quaderni Ital J Med 2017;5(1).

19. De Lillo D. Rumore bianco. Torino: Einaudi 1999. 


\title{
Problematiche organizzative ed assistenziali nella gestione del paziente critico in Medicina Interna
}

\author{
Filippo Pieralli, ${ }^{1}$ Antonio Mancini, ${ }^{1}$ Carlo Nozzoli ${ }^{2}$ \\ ${ }^{1}$ Medicina Interna ad Alta Intensità, AOU Careggi Firenze; ${ }^{2}$ Dipartimento di Medicina Interna ed Emergenza, AOU Careggi \\ Firenze, Italia
}

\section{Che cos'è l'Area Critica in Medicina Interna?}

L'Area critica in medicina interna (ACMI) non ha una definizione standardizzata in letteratura. In pratica definisce la necessità di creare in aree mediche, setting a più elevata intensità di cura che garantiscano standard di cura più elevati rispetto a quelli erogabili in reparti di degenza ordinaria, ma che non abbiano le caratteristiche di Terapia Intensiva.

Nel mondo anglosassone ed in molti paesi europei, la critical care medicine non è una disciplina appannaggio di specialisti in anestesia e rianimazione, ma di medici di varia estrazione specialistica, solitamente medicina interna e pneumologia, che abbiano un curriculum formativo adeguato per gestire i pazienti critici.

Generalmente si fa riferimento a Intermediate Care Units o High Dependency Units come a quelle strutture che offrono il trattamento ed il monitoraggio di pazienti critici definibili come clinicamente instabili con una disfunzione d'organo o a rischio di insufficienza d'organo, ma che non necessitano di ventilazione meccanica invasiva o procedure più avanzate come ossigenazione tramite circuiti extracorporei (ECMO). ${ }^{1,2}$

L'ammissione dei pazienti può avvenire direttamente in ingresso (Step-up) o per trasferimento da terapie intensive (Step-down).

Per entrare nel concreto, le condizioni cliniche che possono necessitare di ammissione in ACMI sono numerose e riportate in Tabella 1.

La caratteristica delle ACMI è quindi la poliva-

Corrispondente: ????? Nome, contatti completi

E-mail: filippopieralli@gmail.com ; nozzolicarlo@gmail.com

Articolo pubblicato secondo la Creative Commons Attribution NonCommercial 4.0 License (CC BY-NC 4.0).

${ }^{\circ}$ Copyright: the Author(s), 2020

Licensee PAGEPress, Italy

QUADERNI - Italian Journal of Medicine 2020; 8(3):29-32 lenza e la capacità di flessibilità capaci di garantire l'adeguamento della domanda e dell'offerta al ricovero di pazienti complessi e polipatologici. L'organizzazione di un ACMI rappresenta ad oggi verosimilmente la risposta più efficiente dal punto vista costo efficacia per la gestione di tali problematiche, superando, con il coordinamento e la gestione omogenea del personale medico ed infermieristico all'interno della stessa area. Uno studio multicentrico europeo $^{3,4}$ ha dimostrato che negli ospedali in cui è presente una Intermediate Care Unit la mortalità ospedaliera dei pazienti ricoverati in terapia intensiva (valutata fino alla dimissione dall'ospedale) è più bassa rispetto agli ospedali privi di queste strutture. La verosimile spiegazione di questo dato sta nel fatto che le strutture di TI sotto pressione per necessità di posti letto trasferiscono precocemente nei reparti di degenza ordinaria che non hanno adeguata capacità gestionale clinica e assistenziale di pazienti ancora troppo complessi per strutture di degenza ordinaria.

Nonostante la loro potenziale e sfavorevole evolutività non tutte le patologie in Tabella 1 necessitano obbligatoriamente di un percorso in Area Critica, bisogna innanzitutto individuare i segnali di allarme, di destabilizzazione emodinamica e delle funzioni d'organo che impongano uno step up del livello di assistenza. A tale proposito è ormai consolidato l'utilizzo di score prognostici in grado di valutare con precisione il rischio di destabilizzazione del paziente in particolare il modified early warning score (MEWS) ed il national early warning score (NEWS) soprattutto quest'ultimo, più accurato nella definizione del rischio e che tra i vari parametri considerati ingloba anche la saturimetria e la necessità di ossigenoterapia. L'implementazione delle varie cartelle informatizzate consente e consentirà sempre più il calcolo veloce dei vari scores anche di più complessi ma importanti come il SOFA score nella sepsi, fornendo alert di facile visione da parte del medico e dell'infermiere e riducendo così il rischio di pericolose sviste. ${ }^{5,6}$

Esistono alcune ragioni a supporto della necessità di ACMI, le principali che possiamo identificare sono le seguenti: i) Nei reparti di Medicina Interna sono ri- 
coverati sempre più pazienti critici e complessi (terapie innovative, possibilità terapeutiche, prolungamento della cronicità); ii) Le Terapie Intensive hanno innalzato le potenzialità terapeutiche per malati sempre più gravi (intubati, ECMO) e non possono farsi carico dei malati meno gravi (rapporto costo efficacia sfavorevole); iii) I pazienti non più bisognosi di ricovero in Terapia Intensiva hanno talvolta bisogno di uno progressivo svezzamento con elevata necessità assistenziale prima di essere assistiti in reparti ordinari (Step Down); iv) La capacità di orientarsi e gestire il paziente complesso e con molteplici comorbidità, rende l'internista il candidato idoneo alla gestione della Critical Care.

\section{Come organizzare un'Area Critica in Medicina Interna?}

Criticità clinica e criticità assistenziale. La rivalutazione dell'assetto organizzativo degli ospedali, caratterizzato dalla progressiva chiusura o riduzione del numero di posti letto nei reparti specialistici e dal sempre maggiore afflusso di pazienti anziani fragili e polipatologici, spesso in condizioni cliniche dominate dalla disfunzione di uno o più organi e richiedenti per tale motivo supporto e monitoraggio, hanno conferito nuovo vigore alle centralità della Medicina Interna nella gestione del paziente e della sua complessità assistenziale. Anche la recente emergenza COVID-19 ha testimoniato il ruolo centrale delle Medicine Interne nel costituire la spina dorsale degli ospedali. La

Tabella 1. Principali condizioni cliniche che possono necessitare di ammissione in Area Critica di Medicina Interna.

- Insufficienza respiratoria acuta ipossiemica e/o ipercapnica

- Sindrome coronarica acuta (NSTEMI)

- Scompenso cardiaco acuto o riacutizzato

- Aritmie iper/ipocinetiche

- Tromboembolismo venoso

- Patologia cerebrovascolare acuta

- Sepsi

- Shock

- Coma iperosmolare iperglicemico

- Chetoacidosi diabetica

- Disordini elettrolitici

- Emorragie acute con instabilità emodinamica

- Pancreatite acuta grave

- Avvelenamenti o Intossicazioni da farmaci

- Paziente post chirurgico complesso

- Politraumi gestione del COVID-19 è stata affrontata principalmente dai Pronto Soccorso, Terapie Intensive e Medicine Interne. In particolare si stima che quest'ultime abbiano gestito circa il 60\% di tutti i ricoveri (fonte survey Centro Studi FADOI). Questo cambiamento dello scenario operativo richiede di conseguenza l'adeguamento ed il rinnovamento delle competenze e della organizzazione dei reparti di Medicina Interna.

L'attuale ruolo delle Medicine Interne, le vede impegnate sempre più nella gestione di pazienti complessi, polipatologici, fragili per condizioni intrinseche o per prolungate ospedalizzazioni anche dopo interventi chirurgici. Si stima che circa il 10-15\% dei pazienti ricoverati in degenza ordinaria di Medicina Interna avrebbe necessità di un setting a più elevata intensità di cura sia per motivi clinici che assistenziali. ${ }^{7,8}$

I pazienti possono avere elevate criticità cliniche e assistenziali o una prevalenza di una delle due. A titolo di esempio un paziente che si ricovera in ACMI per insufficienza respiratoria acuta che necessita di trattamento ventilatorio non invasivo ha elevata criticità clinica per necessità di inquadramento, scelte terapeutiche, necessità di garantire adeguati accessi vascolari venosi o arteriosi, la possibilità di necessitare di drenaggio di un versamento pleurico che incide negativamente sulla funzionalità respiratoria. In altra condizione può essere elevata la criticità clinica ma non assistenziale, ad esempio un paziente con embolia polmonare submassiva che stia effettuando terapia eparinica non endovenosa; oppure elevata criticità assistenziale ma bassa criticità clinica, come nel caso di un paziente post-chirurgico complesso ma stabilizzato che abbia necessità di gestione infermieristica complessa per la presenza di più drenaggi e medicazioni complesse di ferite, oppure ancora un paziente in step-down dalla TI stabilizzato con tracheostomia che richiede competente gestione infermieristica (Figura 1). La gestione operativa dei pazienti non si può limitare al dedicare loro aree solo teoricamente ad elevata intensità di cura, ma il concetto deve essere sostenuto da adeguate risorse mediche, infermieristiche, operatori socio-sanitari e fisioterapisti oltre che da tecnologie e spazi adeguati. Il paziente critico, ovunque esso sia ricoverato, richiede continuo monitoraggio dei parametri vitali, spesso anche invasivi, interventi diagnostico-terapeutici e di nursing tempestivi.

Le criticità organizzative che devono essere affrontate in ambito organizzativo sono di vario tipo che possiamo elencare in maniera sintetica: i) Situazione logistica. Adeguati spazi per pazienti e personale; ii) L'ACMI può essere individuata come area interna al reparto di Medicina Interna oppure come area a se stante preferenzialmente in contiguità con la TI o in spazi comuni di Area critica; iii) Adeguate risorse umane; iv) Adeguate dotazioni strumentali. 
In particolare per le dotazioni strumentali tutti i pazienti devono essere dotati di monitoraggio multiparametrico con centralina visibile da bancone o sistemi telemetrici; devono essere disponibili ventilatori ospedalieri in grado di effettuare ventilazione non invasiva ed in caso di necessità anche invasiva, macchine per la somministrazione di Ossigeno ad alti flussi (HFNC); ecografo facilmente trasportabile con dotazione multisonda; testate con Ossigeno ad alta portata, vuoto e aria compressa. Da non trascurare un adeguato numero di prese elettriche (almeno 6 per letto) per la gestione dei dispositivi elettromedicali, incluso un carrello per emergenza che deve essere sempre disponibile. Infine, è necessaria la rapida disponibilità di emogasalizzatore, meglio se dedicato.

L'adeguata dotazione di risorse umane adeguatamente formate è l'elemento imprescindibile per l'organizzazione di una ACMI. In pratica, per quanto riguarda la dotazione medica l'optimum sarebbe garantire personale dedicato $24 \mathrm{~h} / 7 \mathrm{~g}$ e comunque con un modello minimo dedicato nelle 12 ore diurne.

La dotazione infermieristica è più ben definita, anche dal punto di vista legislativo, e corrisponde in Subintensiva ad 1 infermiere ogni 4 pazienti ed 1 operatore sociosanitario ogni 8 .

\section{Quali competenze sono necessarie per l'internista in Area Critica?}

L'elemento delle risorse umane è fondamentale, ma oltre all'adeguatezza del numero è necessario che il personale che opera in ACMI abbia competenze adeguate per la gestione di pazienti critici e complessi. In una survey promossa nel 2010 da FADOI risultava che le competenze dei medici internisti che disponevano di ACMI era nettamente subottimale (Figura 2). Dalla survey emergeva come meno della metà dei medici fossero autonomi nel posizionamento di un catetere venoso centrale e che solo circa $1 / 3$ fossero autonomi nell'ecografia toracica e cardiaca. La survey ha certamente dei limiti che sono comuni a tutte le indagini a campione e passati 10 anni è molto probabile, anche per l'impegno profuso da FADOI nella formazione di competenze ecografiche, che l'autonomia in ambito ecografico sia notevolmente cresciuta. Da questo punto di vista il tempo è nostro alleato, i giovani internisti hanno sempre maggiore confidenza con l'ecografia in urgenza, la ventilazione non invasiva, le procedure di accesso vascolare, ma resta comunque essenziale pianificare un percorso formativo nella gestione del paziente critico per tutti gli operatori, medici ed infermieri, coinvolti in essa. La nostra società ha sicuramente un ruolo determinante nella promozione culturale del sapere e saper fare, attraverso gruppi di lavoro, corsi e formazione sul campo con la tecnica del learning by doing al fine di promuovere la cultura comune della Critical Care della quale l'internista moderno deve essere interprete e promotore anche attraverso l'integrazione con gli altri attori della critical care in particolare rianimatori, medici di emergenza-urgenza, specialisti che gestiscono pazienti critici (cardiologi, pneumologi, neurologi, chirurghi).

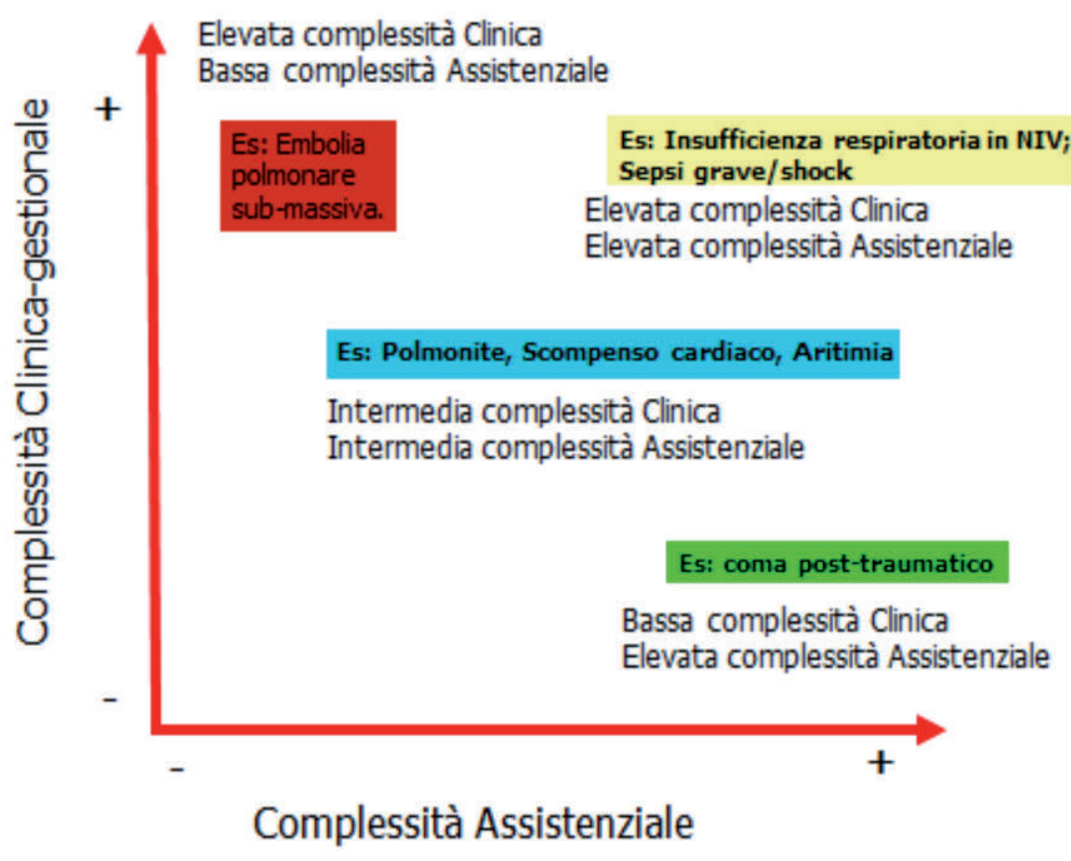

Figura 1. Complessità clinica-gestionale e assistenziale in Area Critica di Medicina Interna. 


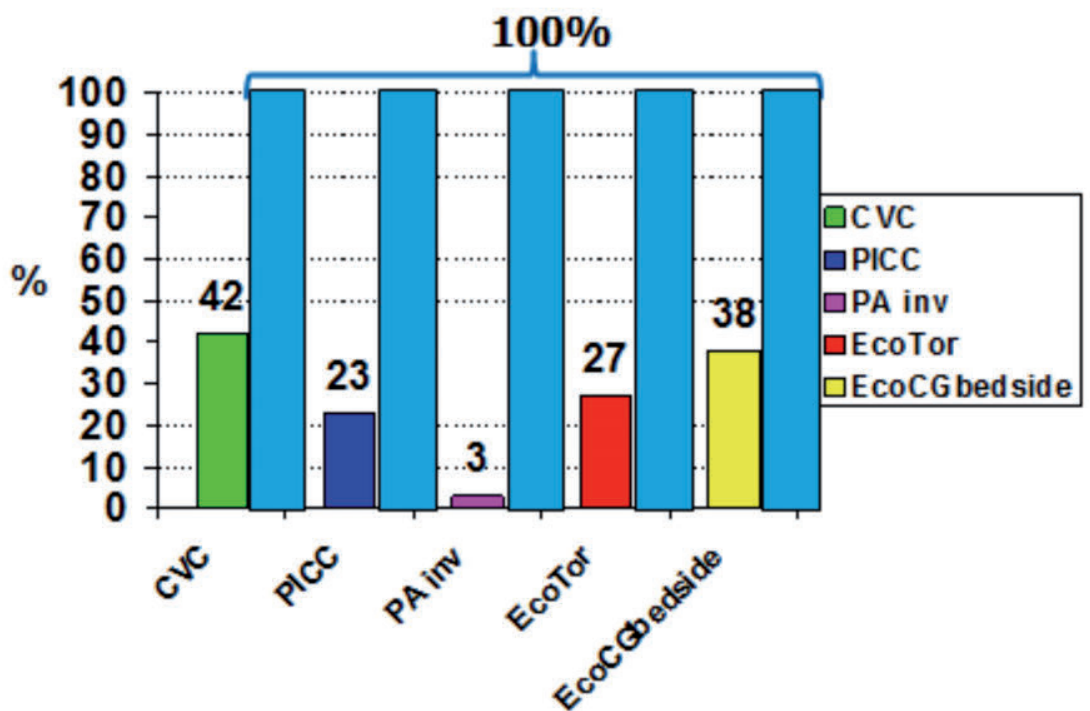

Figura 2. Procedure eseguite in autonomia (survey FADOI 2010).

\section{Conclusioni}

In sintesi la Critical Care è una disciplina che deve essere patrimonio comune del medico internista. La Medicina Interna necessità di aree dove potere gestire in maniera adeguata i pazienti a più elevata complessità clinica e assistenziale che per il profilo di rischio non possono essere adeguatamente gestiti in sicurezza in regime di degenza ordinaria. Le ACMI hanno necessità logistiche ed organizzative che devono rispettare standard minimi di riferimento. La dotazione di risorse umane adeguate per numeri e competenze è un elemento imprescindibile per la creazione delle ACMI al fine di garantire la sostenibilità di modelli che si sono dimostrati efficienti ed efficaci dove messi in atto. FADOI ha sicuramente un ruolo determinante nella promozione culturale del sapere e saper fare al fine di promuovere la cultura comune della Critical Care attraverso l'integrazione con gli altri attori della Critical Care.

\section{Bibliografia}

1. Nasraway SA, Cohen IL, Dennis RC, et al. Guidelines on admission and discharge for adult intermediate care units. American College of Critical Care Medicine of the Society of Critical Care Medicine. Crit Care Med 1998;26:607-10.

2. Rosenthal GE, Sirio CA, Shepardson LB, et al. use of Intensive Care units for Patients With Low Severity of Illness. Arch Intern Med 1998;158:1144-51.

3. Capuzzo M, Volta CA, Tassinati T, et al. Hospital mortality of adults admitted to Intensive Care units in hospitals with and without Intermediate Care units: a multicentre European cohort study. Critical Care 2014;18:551.

4. Simchen E, Sprung CL, Galai N, et al. Survival of critically ill patients hospitalized in and out of intensive care. Crit Care Med 2007;35:449-57.

5. Subbe CP, Kruger M, Rutherford P, Gemmel L. Validation of a modified Early Warning Score in medical admissions. QJM 2001;94:521-6.

6. Sistema Nazionale Linee Guida SNLG - Regione Toscana. National Early Warning Score (NEWS), Misurazione standardizzata della gravità della malattia, Tradotto ed adattato dalla linee guida originale della Royal College of Physician da Berni G, Francois C e Tonelli L; 2014.

7. Nardi R, Arienti V, Nozzoli C, Mazzone A. Hospital organization based on intensity of care: potential errors to avoid. Ital J Med 2012;6:10.4081/itjm.2012.1.

8. Alessandri M, Bartolomei C, Bernardini M, et al. Medicina interna e nuova organizzazione ospedaliera: la proposta della FADOI Toscana. Ital J Med 2007; 1:65-9. 


\title{
I rapporti delle UO di Medicina Interna con il DEA e l'Ospedale
}

\author{
Alessandro Franco, ${ }^{1}$ Giorgio Ballardini ${ }^{2}$ \\ ${ }^{1}$ U.O. Geriatria, Ospedale Infermi di Rimini, AUSL Romagna; ${ }^{2}$ U.O. Medicina Interna, Ospedale Infermi di Rimini, AUSL \\ Romagna, Italia
}

\section{Introduzione}

A partire dagli anni ' 90 il Sistema Sanitario Nazionale è stato interessato dal fenomeno della deospedalizzazione, attraverso il quale il SSN ha programmato la progressiva riduzione dei posti letto di area medica e chirurgica a favore di un incremento delle cure specialistiche extraospedaliere territoriali. Nei primi 10 anni del terzo millennio tale processo ha determinato una riduzione di circa 45.000 posti letto pari al $15 \%$ del totale per cui, fra il 2000 e il 2012, il numero di letti per acuti è passato da 5,1/1000 ab a 3,6/1000 ab (dei quali lo $0,6 \%$ riservato a letti di lungodegenza). ${ }^{1}$

Nel contempo l'Italia ha presentato una crescita della età media della popolazione con aumento delle fasce di età più avanzate e numero di ultraottantenni passato da 2.498 .313 nel 2002 a 4.330 .074 nel 2019 corrispondente ad un incremento del $73 \%$ di ultraottantenni. ${ }^{2}$ Questo fenomeno ha determinato cambiamenti nelle caratteristiche del paziente che si ricovera in area medica: oggi infatti prevalgono pazienti di oltre 75 anni nei reparti internistici e di oltre 80 anni in quelli geriatrici accomunati dalla presenza di complessità clinica e assistenziale. Questi pazienti ormai rappresentano circa la metà dei ricoveri acuti in area medica. ${ }^{2,3}$ Nonostante gli sforzi fatti per ottenere la progressiva riduzione della degenza media registrata in questi anni, l'andamento epidemiologico rappresenta la motivazione (prevedibile) delle criticità negli ospedali e nell'area internistico-geriatrica in particolare.

Corrispondenti: Alessandro Franco, UO di Geriatria, Ospedale "Infermi" di Rimini, Via L. Settembrini 2, 47923, Rimini, Italia. E-mail: alessandro.franco@auslromagna.it

Giorgio Ballardini, UO di Medicina Interna, Ospedale "Infermi” di Rimini, Via L. Settembrini 2, 47923, Rimini, Italia E-mail: Giorgio.ballardini@auslromagna.it

Articolo pubblicato secondo la Creative Commons Attribution NonCommercial 4.0 License (CC BY-NC 4.0).

${ }^{\circ}$ Copyright: the Author(s), 2020

Licensee PAGEPress, Italy

QUADERNI - Italian Journal of Medicine 2020; 8(3):33-38
I fenomeni del sovraffollamento, dell'accumulo di pazienti in attesa di ricovero e della occupazione temporanea di posti letto diversi da quelli di Medicina Interna e Geriatria sono la conseguenza di una sempre più crescente richiesta di aiuto da parte della parte più fragile della popolazione e costituiscono ormai un circolo vizioso che rallenta il processo di input-throughput-output del paziente in PS causando frammentazione del percorso di cura, allungamento dei tempi di degenza, dimissione anticipata, re-ricovero e possibili ripercussioni negative sul paziente (rischio di comparsa/peggioramento della fragilità e riduzione/perdita dell'autonomia residua).

\section{La Medicina Interna nelle organizzazioni ospedaliere}

Il ruolo centrale della Medicina Interna nell'organizzazione di un ospedale è un tema molto discusso da FADOI sia all'interno della nostra società che in ambito istituzionale. L'analisi del problema ha messo in evidenza diverse difficoltà da affrontare con un approccio condiviso dopo aver individuato le priorità clinico-assistenziali ed evitando i percorsi diagnostici frammentati. È indispensabile che a tutti i livelli della piramide, dalle direzioni generali fino allo specialista d'organo, sia chiara la necessità di riconsiderare il ruolo della Medicina Interna e della Geriatria riconoscendo la figura dell'internista/geriatra come riferimento della complessità all'interno dell'ospedale. Del resto le linee guida internazionali suggeriscono da tempo di sostituire nei DEA la focalizzazione sulla patologia con una gestione centrata sulla valutazione multidimensionale in grado di rendere manifesti i bisogni individuali del paziente anziano. ${ }^{4} \mathrm{Nel}$ Regno Unito è in corso un programma gestionale denominato Future Hospital, volto a concentrare le aree cliniche all'interno di una Acute Medical Unit con l'obiettivo di trattare l'acuzie entro $48 \mathrm{~h}$, contrastare il sovraffollamento dell'area di emergenza e programmare per tempo il trasferimento del paziente stabile alle cure territoriali. $^{5}$

La chiara dimostrazione di un peso negativo della degenza ospedaliera prolungata e dei ricoveri ripetuti 
sul paziente complesso e fragile induce a reinventare, nel nostro sistema sanitario, l'organizzazione ospedaliera fra reparti di Medicina Interna/Geriatria e DEAPS, affinché si identifichino ed implementino nuovi modelli organizzativi capaci di contrastare il sovraffollamento, facilitando, nel contempo, l'integrazione fra ospedale e territorio. Negli ultimi anni, fra le proposte sperimentate in Italia, i modelli ospedalieri più utilizzati sono stati il modello ospedaliero organizzato per intensità di cura ${ }^{6,7}{ }^{6}$ 'Osservazione Breve, ${ }^{8}$ sia nella variante osservazione breve intensiva in PS che osservazione breve internistica annessa alla UO di Medicina, e il Day Service polispecialistico ${ }^{9}$ con accesso direttamente da PS.

Il Modello per intensità di cure prevede un'area di degenza medica, distinta da PS e Medicina d'Urgenza, ove allocare pazienti sulla base della intensità di cure necessaria, indipendentemente dalle eventuali patologie prevalenti, con successivo trasferimento in aree a più bassa intensità una volta raggiunta la stabilità clinica del paziente. Tale modello è entrato rapidamente in difficoltà a causa di problematiche legate alla logistica (spazi adeguati per letti altamente dinamici) e alla difficoltà di controllare posti letto a diversa intensità assistenziale nel momento in cui si manifestano sovraffollamento e lunghi tempi di attesa in PS. ${ }^{10}$

L'osservazione breve prevede un'area intermedia tra Pronto Soccorso e UO di Medicina/Geriatria ove gestire pazienti con acuzie intermedia per i quali non è evidente la necessità di ricovero in degenza ordinaria ma occorre impostare un iter diagnostico terapeutico e di stabilizzazione clinica per poi indirizzare il paziente al territorio. In particolare, essa gestisce emergenze-urgenze per problemi clinici acuti ad alto grado di criticità ma a basso rischio evolutivo oppure a basso grado di criticità ma con potenziale rischio evolutivo con iter diagnostico-terapeutico non differibile. Questo servizio ricalca l'organizzazione della Clinical Decision Unit (CDU) anglosassone ${ }^{11}$ che prevede letti con monitoraggio multi-parametrico, nel contesto del DEA, dove afferiscono pazienti internistici a bassomedio rischio (scale di valutazione MEWS) valutati dall'internista che svolge l'intervento diagnostico-terapeutico entro $48-72 \mathrm{~h}$, stabilizza la fase acuta, esclude patologie complesse che richiedano il ricovero e dimette entro $72 \mathrm{~h}$, riaffidando il paziente alla rete territoriale.

Il Day Service, che è invece nato come presidio di cura programmato nel quale indirizzare pazienti con una patologia prevalente e un percorso di diagnosi e cura predefinito, ha le potenzialità organizzative per trattare problematiche mediche non severe, dopo inquadramento clinico in PS, in un setting ambulatoriale dotato di accesso preferenziale alla diagnostica strumentale. Il governo del Day Service si fonda sull'uso di PDTA specifici per tipologie cliniche quali lo scompenso glicometabolico nel paziente stabile, lo scompenso cardiaco di grado moderato, le polmoniti di comunità e la BPCO riacutizzata. L'accesso a questo presidio, che manifesta la migliore efficacia in strutture ospedaliere il cui DEA/PS non sia dotato di osservazione breve, avviene dopo consulenza internistica in PS e prevede la gestione del paziente nelle $24 \mathrm{~h}$ con affidamento in strutture territoriali (ambulatori della cronicità, servizi diabetologici, ambulatorio dello scompenso cardiaco) dove viene garantita la continuità della cura. Con questa dizione il confine fra Day Service e Osservazione Breve internistica appare sfumato.

Uno strumento ulteriore per migliorare la relazione fra PS e attività nelle U.O. mediche è rappresentato dalla consulenza internistico/geriatrica. In letteratura è documentato che la consulenza geriatrica in PS con applicazione del Comprehensive Geriatric Assessment (CGA) ${ }^{12}$ è in grado di ridurre il rischio di ricovero improprio e la letteratura rimarca la significatività di questo risultato soprattutto per chi accede in PS per dolore toracico e muscolo scheletrico. ${ }^{13}$ Risultati positivi sono stati osservati anche nel caso di anziani accolti in PS con problematiche socio-assistenziali prevalenti e outcome rappresentato da riduzione della degenza media e del re-ricovero. ${ }^{11}$ Il CGA tuttavia complica, perché complesso, la consulenza geriatrica e andrebbe preceduto da strumenti di screening più pratici. $\mathrm{Al}$ di là di una semplice consulenza intesa come parere professionale dello specialista d'organo, occorrerebbe poter garantire anche una presa in carico globale del paziente.

I modelli gestionali predetti richiedono un team professionale (hospitalist, bed manager, medico specialista ospedaliero, ecc.) dotato di competenze per fornire un flusso appropriato di pazienti in base all'urgenza (colori del triage) e per individuare eventuali percorsi alternativi al ricovero. Il team dovrebbe anche essere in condizioni di raccordarsi con la rete territoriale per la continuità della cura una volta superata la fase acuta che ha portato al PS.

\section{La realtà nel nostro paese}

Nella realtà italiana i problemi di sovraffollamento del PS nell'attesa di accedere al reparto e quello delle dimissioni difficili dall'area medica sono gestiti in maniera non uniforme anche se gli strumenti per affrontarli spesso coincidono. Le azioni intraprese più spesso sono state: i) contatti quotidiani fra PS e reparti medici per definire la disponibilità di posti letto; ii) creazione di posti letto con spostamento di pazienti fra reparti, utilizzo della discharge room e dimissioni anticipate al mattino; iii) ricoveri di pazienti stabili in appoggio in letti di altri reparti ma, comunque, in carico alla Medicina/Geriatria; iv) consulenza internistico/geriatrica in PS; v) istituzione di aree polmone per ridurre il carico di lavoro nella UO e limitare il fenomeno dei pa- 
zienti in appoggio; vi) blocco dei ricoveri programmati; vii) istituzione di reperibilità medica ed infermieristica straordinaria per gestire i carichi di lavoro aumentati.

L'analisi delle azioni intraprese negli ultimi anni in Italia ha individuato esempi di intervento che si riportano di seguito.

Il Piano Strategico di gestione del Policlinico di Bari ${ }^{14}$ prevede, in caso di eccesso di pazienti in attesa in PS, l'istituzione di percorsi specifici in modalità fasttrack e l'implementazione del Nucleo Assistenziale Avanzato per la gestione dei codici urgenti differibili (1 h), urgenti minori (2 h) e non urgenti (4 h) gestiti da un team di valutazione clinico-assistenziale (medico e infermiere) per decidere il percorso appropriato (ricovero, dimissione protetta, dimissione a domicilio).

Nella Azienda Ospedaliera G. Brotzu di Cagliari il Piano di Gestione del sovraffollamento di pazienti internistici e chirurgici ${ }^{15}$ prevede interventi soprattutto nelle fasi di throughput e output piuttosto che di input attraverso percorsi prioritari per prestazioni in urgenza, attività di consulenza internistica/geriatrica in PS, ricorso a letti in appoggio o sovrannumero e modifica temporanea dei letti da valenza DH/DS a degenza ordinaria, unitamente a strategie di rinforzo del personale. In caso di esaurimento dei letti è prevista la deviazione dei pazienti che accedono tramite 118 in altro presidio.

Analoghe misure in base ai livelli di criticità dei flussi in input si osservano in Toscana ${ }^{16} \mathrm{e}$ in Sicilia. ${ }^{17}$ Nel Lazio ${ }^{18}$ sono stati adottati un sistema informativo denominato TELEMED (trasmissione di ECG da 118 a PS nel sospetto di SCA e in altre emergenze mediche), programmi di miglioramento del comfort in PS (siamo qui con te e accorciamo le distanze), percorsi di continuità assistenziale multiprofessionale fra PS e UO mediche per il ricovero appropriato del paziente acuto (pool di medici internisti h 24 per gestire il flusso dei ricoveri in ambiente medico, valutazione internistica in PS) e dimissione da UO controllata attraverso la holding area (che raccorda con la rete dei servizi territoriali). Le dimissioni avvengono 7/7 giorni, in linea con l'attività del PS, ed è attivata una dimissione in codice 5 (il paziente è dimesso e torna per completare l'iter diagnostico senza essere ricoverato nuovamente). Potenziati sia i collegamenti informatici con case della salute e ambulatori di cure primarie dei MMG che quelli fra i dati del PS e il Fascicolo Sanitario Elettronico.

Si segnala, inoltre, una esperienza piemontese di valutazione geriatrica nel PS dell'Ospedale Mauriziano di Torino dove è stato implementato un ambulatorio di valutazione geriatrica per la presa in carico di pazienti con bassa intensità clinica ed elevata intensità assistenziale, per i quali viene adottato un programma di follow-up stretto volto a ridurre il sovraffollamento, le lunghe attese per ricovero e reaccesso in PS. L'analisi dei dati preliminari (non ancora disponibili) potrebbe fornire informazioni utili sul modo di discriminare i pazienti che non traggono giovamento da un ricovero ospedaliero rispetto a quelli che ne abbiamo, al contrario, bisogno. ${ }^{19}$

In Emilia Romagna il sovraffollamento è stato affrontato in diverse realtà aziendali con strumenti di valutazione applicabili nelle varie fasi del percorso di accoglienza all'interno del DEA. Nei PS vengono implementati modelli matematici, ad esempio il NEDOCS, per la misurazione di indicatori statici e dinamici di sovraffollamento. ${ }^{20}$ Viene sperimentato uno score del rischio di morte o ricovero entro l'anno denominato RISKER. ${ }^{21-23}$ Questo algoritmo, alimentato da database esistenti ed applicato su tutta la popolazione, nasce come strumento per MMG e case della salute per distinguere il grado di rischio sulla popolazione e implementare progetti di intervento territoriale dedicati ai sottogruppi ad alto rischio, con l'obiettivo di prevenire i ricoveri.

I tentativi predetti dovrebbero essere propedeutici a nuovi assetti organizzativi atti a garantire un rapido inquadramento clinico-assistenziale del paziente complesso in PS, definire i bisogni e favorire l'accesso rapido alla diagnostica/consulenza specialistica, indirizzando il paziente, quando possibile, verso soluzioni alternative al ricovero. A questo proposito la AUSL della Romagna ha da tempo sviluppato un percorso aziendale omogeneo per la gestione delle dimissioni protette che si avvale del lavoro svolto da un team rappresentato da bed manager (figura professionale di solito infermieristica), internista/geriatra e nucleo di Continuità Ospedale-Territorio (NUCOT). ${ }^{24} \mathrm{Al}$ team compete il monitoraggio continuo dei posti letto giornalieri per allocare i pazienti del PS che attendono il ricovero o la dimissione protetta nel setting appropriato. Questo progetto ha dimostrato di sostenere la personalizzazione delle cure e la riduzione della frammentazione degli interventi nel paziente con complessità e fragilità.

\section{Cosa pensano gli internisti dei rapporti con il PS}

Recentemente FADOI ha condotto una survey fra i propri soci che contiene items legati al rapporto fra DEA e ospedale (Pastorelli R, Survey Un giorno in Medicina. XXIV Congresso Nazionale FADOI. Firenze, maggio 2019; fadoi.org). L'indagine ha fatto emergere diverse opinioni comuni nel mondo internistico fra le quali la percezione di un ruolo modesto dell'internista nei processi decisionali che si svolgono quotidianamente in ospedale. Il 50\% degli intervistati è convinto che il collega dell'emergenza, di fronte ad un paziente con problematiche mediche di tipo acuto accolto in PS, ritenga sufficiente il solo coinvolgimento dello specialista d'organo per garantirne l'inquadramento esclu- 
dendo il medico con competenze internistico- geriatriche. A riprova di ciò vi è il dato che circa il $60 \%$ dei pazienti acuti, prima del ricovero in Medicina o Geriatria, viene valutato dallo specialista d'organo ma non dall'internista. Il mancato coinvolgimento va purtroppo considerato come una grave lacuna nell'organizzazione gestionale dei rapporti fra DEA e UO mediche laddove, invece, un percorso condiviso migliorerebbe il flusso di ricoveri favorendo una concomitante riduzione del rischio di ricovero inappropriato che, dalle risposte fornite, sembra verificarsi in circa un terzo dei pazienti ricoverati. La mancata collaborazione in PS fra medico dell'urgenza ed internista si traduce, secondo il $40 \%$ dei colleghi, nella sensazione che la rilevanza della propria UO di Medicina nell'organizzazione ospedaliera venga considerata modesta e, nel 22\% dei casi, addirittura ancillare il ruolo svolto dall'internista nel processo decisionale del paziente acuto. E ancora, in oltre il $90 \%$ delle risposte fornite nella survey, si percepisce il bisogno degli internisti di collaborare con il medico di PS e, eventualmente, con altre figure specialistiche, nel percorso di allocazione del paziente nel setting adeguato (reparto internistico/geriatrico, OBI, holding area ecc.).

Se si prendono in considerazione le risposte fornite nella valutazione dei rapporti fra il mondo internistico ed il resto dell'ospedale la nostra impressione è che gli altri specialisti abbiano una visione non forte delle UO internistiche/geriatriche rispetto ad altre unità operative. Non sembra che alla consulenza internistica venga attribuita la stessa rilevanza clinica rispetto ai pareri degli specialisti d'organo, soprattutto nei casi di incertezza clinica e di dubbi sui percorsi gestionali appropriati. Del resto, non infrequentemente, anche le direzioni strategiche aziendali sembrano non avvertire l'opportunità di coinvolgere l'internista nello sviluppo di percorsi clinici e gestionali ospedalieri/territoriali.

Un'ultima considerazione emergente dall'analisi dei dati della survey è la percezione che alla Medicina Interna non venga riconosciuta, all'interno dell'organizzazione ospedaliera, quella solida e compatta competenza e capacità organizzativa che di prassi viene attribuita agli altri reparti specialistici. Ciò, probabilmente, incoraggia comportamenti non adeguati come, ad esempio, la consuetudine che il medico del PS, in caso di dubbio clinico, si adatti più facilmente alle decisioni dello specialista d'organo trovando più praticabile forzare la presa in carico del paziente borderline da parte della Medicina Interna piuttosto che assegnare il paziente ad altro reparto, ponendo quest'ultimo a rischio di ricovero in setting non appropriato.

\section{Conclusioni}

Dall'analisi della survey e considerando le diverse possibilità prospettate per la soluzione delle problematiche che affliggono i DEA in Italia, i punti di ri- flessione sono numerosi e complessi. Se si tiene presente che la Medicina Interna dovrebbe rappresentare il tessuto connettivo all'interno dell'ospedale, affinché ciò si realizzi, è prima di tutto necessario che cresca la consapevolezza del ruolo dell'internista fra gli stessi internisti, che purtroppo, non raramente, si considerano specialisti d'organo mancati e che, quelle che oggi sono considerate variabilità e differenze, da limiti, divengano punti di forza. Proprio la variabilità è ciò che consente al medico di Medicina Interna di adattarsi ed affrontare le situazioni nuove ed inusuali, di rimboccarsi le maniche per applicare metodo e ragionamento clinico quando lo specialista d'organo o di malattia, dichiara la propria non competenza. Il ruolo della Medicina Interna e della Geriatria nella gestione dei pazienti COVID sta rappresentando, senza ombra di dubbio, un esempio di impegno e di capacità di reinventarsi quotidianamente sia dal punto di vista clinico che organizzativo.

I principi dell'appropriatezza clinica, diagnostica e terapeutica, associati agli strumenti della clinical governance, devono pertanto diventare il know-how dell'internista per consentirgli di assumere, nel contesto di un team formato anche dallo specialista d'organo, il ruolo di regista e leader della complessità all'interno della struttura ospedaliera la cui gestione, di fatto, rappresenta il vero problema all'interno di un DEA.

Altro punto fondamentale è rappresentato dalla capacità di integrazione dell'internista con la rete del territorio intesa sia come presidi territoriali che come ospedali di comunità. L'individuazione di figure professionali in grado di lavorare con la rete territoriale fra gli internisti ed i geriatri ospedalieri permetterebbe di colmare il gap di collegamento fra l'ospedale ed il medico di medicina generale e di garantire al paziente fragile la continuità assistenziale che merita. Se questa attività centrata sull'internista è più facilmente implementabile negli ospedali di piccole dimensioni, la sua realizzazione risulta certamente più complessa negli ospedali di maggiori dimensioni dove sarebbe più opportuno concentrarsi sul paziente complesso acuto ed organizzare in un secondo tempo la presa in carico nell'area territoriale di riferimento per garantire il proseguimento dell'assistenza.

È evidente che la creazione di percorsi diagnostici preferenziali per particolari tipologie di pazienti o per specifiche malattie, non deve avvenire a scapito di chi non rientra in questi contesti. Nei momenti di sovraffollamento questo non deve incidere sulla gestione ordinaria dei pazienti ricoverati, pena l'allungamento dei tempi di degenza e quindi la vanificazione degli sforzi.

Riassumendo, queste le linee di intervento che sembrerebbero praticabili: i) Accordo interdipartimentale per la gestione in comune della fase di ricovero del paziente complesso/fragile fra medico di PS, in- 
ternista e/o geriatra e bed manager al fine di migliorarne l'appropriatezza formulando sin dall'ingresso in reparto una linea di intervento diagnostico-terapeutica ed una bozza di programma di continuità assistenziale condivise con l'obiettivo di facilitare la dimissione del paziente e la eventuale presa in carico territoriale, soprattutto nel caso che il ricovero sia in modalità fuori reparto; ii) Accordo fra tutte le unità operative ospedaliere per il rientro quanto prima possibile del paziente fuori reparto nella unità operativa di appartenenza al fine di ridurre il carico assistenziale e il rischio clinico correlato alla modalità stessa; iii) Possibilità di riallocare le risorse umane, per garantire un rapporto fra numero di pazienti e medici responsabili appropriato (1 medico ogni 10-12 pazienti); iv) Implementazione di un programma di riduzione della degenza media basato su punti fermi che garantiscano la priorità ai pazienti ricoverati in attesa di eseguire esami strumentali, la consulenza specialistica tempestiva (entro $24 \mathrm{~h}$ ), la realizzazione di liste preferenziali per gli esami post-dimissione e la attivazione precoce del percorso dimissioni difficili; v) anticipazione della dimissione del paziente dal reparto al mattino ed utilizzo della discharge room; vi) implementazione di un ambulatorio internistico/geriatrico, anche con condivisione delle risorse umane fra internisti e geriatri, dedicato a pazienti del PS, selezionati dal triage, che non necessitano di ricovero ospedaliero ma per i quali è necessario impostare un programma di presa in carico per fornire risposte adeguate ai bisogni assistenziali; vii) percorsi diagnostico-terapeutici per i DRG a rischio di inappropriatezza; viii) formazione medica/infermieristica sull'appropriatezza del ricovero; ix) rimodulazione triennale in base ai volumi di attività della distribuzione dei posti letto fra area medica e area chirurgica.

Si confermano le ovvietà che il sovraffollamento e lo sforzo che serve per affrontarlo, non possono essere solo responsabilità del DEA e dell'area internistico-geriatrica ma devono coinvolgere tutta la rete ospedaliera. Come ulteriore riflessione, il contesto attuale, che vede il progressivo incremento dell'area della poli-patologia e della fragilità, coinvolgendo anche l'area degli specialisti d'organo e l'area chirurgica, richiederebbe un re-bilanciamento delle dotazioni di letti e di personale dei diversi ambiti. Ma questo incremento è avvenuto nel giro di pochi anni e così rapidamente da non aver consentito un equilibrio. Serve quindi una inversione di quel modo di pensare che vedeva meno chirurghi ma tutti in sala operatoria e gli internisti a gestire il reparto. Serve una contaminazione, un recupero di capacità da parte degli specialisti di praticare anche i fondamentali della medicina, eventualmente con la consulenza di internisti e geriatri, pena l'incapacità, in attesa di un riequilibrio del sistema, di far fronte alle nuove esigenze.

\section{Bibliografia}

1. Inchiesta Ospedali al collasso. Da Annuario Statistico del SSN. Quotidiano Sanità 2012.

2. Fonte ISTAT. Annuario Statistico Italiano 2017

3. www.tuttitalia.it

4. Heeren P, Devriendt E, Fieuws S et al Unplanned readmission prevention by a geriatric emergency network for transitional care (URGENT): a prospective beforeafter study. BMC Geriatrics 2019; https://doi.org/ 10.1186/s12877-019-1233-9

5. Future Hospital Commission: Caring for medical patient. Royal College of Physicians; 2013. www.rcplondon.ac.uk/futurehospital

6. Mongardi M, Bassi E, Di Ruscio E. Ospedale per intensità di cura: strumenti per la classificazione dei pazienti. RER, Direzione Generale sanità e politiche sociali; Febbraio 2015.

7. Panigada G, Chiti I. Aspetti clinico-organizzativi nella degenza medica ospedaliera in Italia: il ruolo della Medicina Interna nel dipartimento medico e continuità assistenziale. Ital J Med 2015;3:499-502.

8. Ministero della Salute. Linee di indirizzo nazionali sull'osservazione breve intensiva. http://www.salute. gov.it/imgs/C_17_notizie_3849_listaFile_itemName_0_ file.pdf

9. RER e Ministero della Salute. Evoluzione del sistema DRG nazionale. Milestone 1.2.1. Forme alternative alla degenza definizione (day hospital, day surgery, day service) e relativi flussi informativi. http://www.salute.gov. it/imgs/C_22_AttivitaMattoni_19_documenti_documento_0_file $\bar{A}$ llegato.pdf

10. Nardi $\bar{R}$, Arienti V, Nozzoli C, Mazzone A. Organizzazione dell'ospedale per intensita di cure: gli errori da evitare. Ital J Med 2012;6:1-13.

11. Hassan TB. Clinical decision units in the emergency department: old concepts, new paradigms, and refined gate keeping. Emerg Med J 2003;20:123-5.

12. Jay S, Whittaker P, Mcintosh J, Hadden N. Can consultant geriatrician led comprehensive geriatric assessment in the emergency department reduce hospital admission rates? A systematic review. Age Ageing 2017;46:366-72.

13. JJ McCabe, SP Kennelly. Acute care of older patients in the emergency department: strategies to improve patient outcomes. Open Access Emerg Med 2015:7.

14. Piano strategico di gestione dei posti letto aziendali in condizioni di sovraffollamento del PS. Regione Puglia, Azienda Ospedaliero-Universitaria consorziale Policlinico di Bari. Luglio 2019G. Ruggiano. https:/www.sanita.puglia.it/web/ospedalegiovannixxiii/news-in-archivio _det/-/journal_content/56/36067/piano-strategico-gestione-posti-letto-aziendali-in-condizioni-ordinarie-estraordinarie

15. Piano di gestione del sovraffollamento dei pazienti internistici e chirurgici. AO G. Brotzu, delibera n. 158 del 23.01.2019. https://www.aobrotzu.it/documenti/9_204 20190124124414.pdf

16. Piani aziendali per la gestione del sovraffollamento in PS (PGSA). Regione Toscana 2017. https://www.frgeditore.it/images/cop/pdf/titolo-1/razionalizzazione/toscana/all_dgr_974

17. Linee di indirizzo per la gestione del sovraffollamento 
nelle strutture di pronto soccorso della Sicilia; 2018 https:/www.qualitasiciliassr.it/sites/doc/umanizzazione/direttive/ps/D.A.n.1584_del_10.09.2018\%20.pdf

18. Piano regionale per la gestione del sovraffollamento in PS. Decreti del Commissario ad Acta. Regione Lazio 30 ottobre 2019. https://www.regione.lazio.it/binary/rl_sanita/tbl_contenuti/DCA_n._U00453_del_30.10.2019_pi ano_sovraffollamento.pdf

19. Reano A, Balzaretti P, Aurucci ML, et al. Ambulatorio di rivalutazione geriatrica per pazienti dimessi dal pronto soccorso. Uno studio Pilota. Psicogeriatria, Anno XV N 1, pg 120, gennaio-aprile 2020. https://www.psicogeriatria.it/usr_files/pubblicazioni/riviste/psicogeriatria-n1-2020.pdf

20. Linee di indirizzo per la gestione del sovraffollamento nelle strutture di Pronto Soccorso della Regione Emilia Romagna. http://bur.regione.emilia-romagna.it/bur/ area-bollettini/bollettini-in-lavorazione/dicembre-periodico-parte-seconda-1a-quindicina.2017-12-12. 4371406139
21. Louis DZ, Robenson M, McAna J, et al. Predicting risk of hospitalisation or death: a retrospective populationbased analysis. BMJ Open 2014;4:e005223.

22. Pandolfi P, Collina N, Marzaroli P, et al. Sviluppo di un modello predittivo di decesso o ricovero d'urgenza per l'individuazione degli anziani fragili. Epidemiol Prevenz 2016;40:395-403.

23. Morsilio F, Nobilio L, Moro Ml. Stratificazione della popolazione in relazione al rischio di ospedalizzazione per cause prevenibili e decesso: l'algoritmo RiskER. Agenzia sanitaria e sociale regionale dell'ER. http://assr.regione.emilia-romagna.it/pubblicazioni/rapporti-documenti/report-risker-2018

24. AUSL della Romagna. Ravenna 13 ottobre 2018: Continuità ospedale territorio: dimissioni protette e strutture intermedie. https://www.auslromagna.it/cura/formazione/formazione-mmg-pls/materiali-corsi-formazione/2018/formazione-mmg-ravenna-continuita-osped ale-territorio-dimissioni-protette-e-strutture-intermedie?format $=$ html 


\title{
La Medicina Interna: ponte naturale fra Ospedale e Territorio
}

\author{
Tiziana Marcella Attardo, ${ }^{1}$ Stefano De Carli, ${ }^{2}$ Roberta $\mathrm{Re}^{3}$ \\ ${ }^{1}$ UOC Medicina Interna Ospedale Barone Lombardo, Canicattì (AG); ${ }^{2}$ SOC Medicina Interna, Presidio Ospedaliero S. Antonio, \\ S. Daniele del Friuli, Azienda Sanitaria Friuli Centrale (ASU-FC), Udine; ${ }^{3}$ Medicina Interna, ASL Vercelli, Presidio Ospedaliero \\ Sant'Andrea Vercelli, Italia
}

\section{Introduzione}

Il miglioramento delle condizioni sociosanitarie, l'aumento della sopravvivenza a condizioni cliniche un tempo fatali e l'invecchiamento della popolazione hanno portato progressivamente a una profonda modifica dello scenario di cura, con un progressivo incremento delle malattie ad andamento cronico, spesso presenti contemporaneamente nello stesso individuo. $^{1-5}$

Pertanto attualmente un obiettivo sanitario prioritario è rappresentato dalla gestione del malato cronico e dalla definizione di percorsi assistenziali in grado di prendere in carico l'individuo nel lungo termine e prevenirne la disabilità, garantendo, quindi, sia la continuità assistenziale ospedale-territorio, sia l'integrazione degli interventi sociosanitari.

Sempre più frequentemente, le corsie ospedaliere vengono occupate da pazienti anziani o molto anziani, poco o nulla autosufficienti, con malattie croniche riacutizzate, comorbidità, complicanze e postumi invalidanti. In questa tipologia di pazienti, è evidente che la capacità di recupero dello stato di salute dopo un evento acuto è fortemente contrastata e rallentata dall'età avanzata, dalle comorbidità, dalle disabilità, dai devices di cui sono spesso portatori e dalle frequenti complicanze che contraddistinguono il decorso clinico. Sempre più spesso accade, poi, che, nonostante il buon risultato clinico ottenuto durante il ricovero, la dimissione venga ritardata da problematiche di natura extrasanitaria. In particolare, la famiglia che dovrebbe accogliere il paziente ed as-

Corrispondente: Tiziana Marcella Attardo, UOC MCAU Ospedale Barone Lombardo, Canicattì (AG), Italia. E-mail: claudia.fantucchio@alice.it

Articolo pubblicato secondo la Creative Commons Attribution NonCommercial 4.0 License (CC BY-NC 4.0).

${ }^{\circ}$ Copyright: the Author(s), 2020

Licensee PAGEPress, Italy

QUADERNI - Italian Journal of Medicine 2020; 8(3):39-54 sisterlo a casa, o non esiste (anziani soli, senza figli o discendenti diretti) o non è in grado di svolgere questo compito e/o rifiuta di farlo o perché tutti i componenti della famiglia sono impegnati in attività lavorative e/o scolastiche a tempo pieno o, ancora, perché l'assistenza al paziente è comunque troppo gravosa ed impegnativa e richiede competenze e abilità particolari. A ciò si aggiunge il timore da parte dei pazienti di essere abbandonati e non ricevere più lo stesso tipo di assistenza continuativa garantita in ospedale. Inoltre, a volte, il domicilio del paziente non è più adatto a riceverlo a seguito dei suoi postumi invalidanti, spesso necessitante di interventi di ristrutturazione lunghi e costosi.

La dimissione clinica, di conseguenza, non è l'unico criterio che definisce la dimissibilità di un paziente. ${ }^{6-12}$

La dimissione, infatti, rappresenta l'anello debole del passaggio della persona assistita da un servizio ad un altro. Essa quindi è l'aspetto cruciale del piano di assistenza che risponde al diritto della persona e della sua famiglia ad essere coinvolti ed accompagnati in ogni fase della malattia. La dimissione difficile, propria dei cosiddetti pazienti complessi, rappresenta un problema clinico, organizzativo e sociale, sempre più frequente e grave, la cui risoluzione non può che risiedere in una maggiore integrazione tra ospedale e territorio. Ciò significa migliore utilizzo di tutte le opzioni organizzative, ospedaliere e territoriali, disponibili, in un'ottica di rete, cioè di comunicazione ed interazione continua fra tutti gli attori della catena assistenziale. Da qui nasce la necessità di dimissioni protette il cui esito sono delle dimissioni concordate e programmate che garantiscano ai pazienti la continuità assistenziale ed il rientro tutelato al proprio domicilio e alle famiglie il supporto nella gestione del carico assistenziale. ${ }^{13-16}$

D'altra parte, l'ottimizzazione del consumo delle risorse, specie in un momento come questo di crisi economica, impone che l'ospedale per acuti assuma in misura sempre più accentuata il ruolo di risolutore delle fasi epicritiche di una malattia e rappresenti il luogo di concentrazione tecnologica e di sapere spe- 
cialistico. Dunque l'ospedale visto come risorsa estrema da usare solo quando è indispensabile e per il tempo strettamente necessario, ideato ed organizzato ponendo al centro il paziente, con la sua esigenza di cura ed i suoi bisogni assistenziali. Elemento fondamentale che emerge dall'analisi dei bisogni del paziente è che spesso in Sanità si operi in modo discontinuo; il medico spesso offre un'assistenza sanitaria eccellente ma non coordinata, che comporta a volte una sovrapposizione di ruoli ed un'inutile ripetizione d'indagini, che incidono tra l'altro sull'aumento della spesa sanitaria (Tabella 1).

Da una valutazione dello status si evidenzia che il paziente si trova ad usufruire di una assistenza scollegata, per cui è indispensabile creare una relazione tra tutti gli attori e, soprattutto, capire chi fa che cosa.

La mancanza di relazione tra Ospedale e Territorio è una delle criticità che ostacola il corretto percorso assistenziale del paziente, capace di condizionarne il decorso della malattia, generando, inoltre, nel paziente la percezione di inefficienza tecnico-gestionale del Sistema Sanitario. Di seguito vediamo un fotografia real life delle tre Medicine di tre Ospedali italiani posti in tre ambiti territoriali molto differenti: quella agrigentina, quella vercellese e quella udinese e dei loro sistemi di integrazione con la sanità territoriale.

\section{Focus sull'organizzazione Sanitaria in Sicilia e nella provincia di Agrigento}

Il Piano Nazionale della Cronicità (PNC), di cui all'Intesa Stato - Regione n. 160 del 15 settembre 2016, ha individuato sulla base della rilevanza epidemiologica, della gravità, invalidità, difficoltà di diagnosi ed accesso alle cure e peso assistenziale ed economico, le prime patologie croniche su cui focalizzare gli interventi in materia. ${ }^{17}$

La Regione Sicilia, nella consapevolezza dell'im- portanza della presa in carico dei pazienti cronici complessi quale elemento strategico ai fini della sostenibilità del SSR, ha consolidato nel corso degli anni la definizione e l'implementazione di PDTA specifici per singola patologia. ${ }^{17}$

Con la Legge Regionale 14 aprile 2009, n 5 Norme per il riordino del Servizio Sanitario Regionale $^{18}$ e con il Decreto 726 del 10 marzo 2010 Linee Guida sulla Riorganizzazione dell'attività Territoriale $^{19}$ sono stati impartiti gli indirizzi e gli strumenti tramite cui realizzare l'obiettivo in argomento che non può prescindere dalla effettiva interazione ospedale - territorio alla quale entrambi, ospedale e territorio, devono concorrere in modo coerente.

Nell'ambito delle linee programmatiche espresse nel Piano Regionale sanitario (PSR) 2017 - 2019 della Regione Sicilia, ${ }^{20}$ nel capitolo della gestione delle cronicità, è stato posto l'accento sulla necessità di una concezione di un disegno complessivo che attribuisca una centralità reale alla persona attraverso la promozione di modelli assistenziali ed organizzativi innovativi e flessibili in grado di consentire una gestione integrata efficiente ed efficace della cronicità nella sua interezza.

In Sicilia la popolazione residente (dati aggiornati al primo gennaio del 2009) risulta di 5.037.799 unità, di cui 2.433 .605 (48\%) uomini e 2.604 .194 (52\%) donne. ${ }^{21}$ La Regione, la più vasta del Paese (25.711 $\mathrm{km}^{2}$ ), oltre ad essere la più grande isola del Mediterraneo, comprende anche gli arcipelaghi delle Eolie (o Lipari), delle Egadi e delle Pelagie, nonché dalle isole di Ustica e Pantelleria, realtà che pongono peculiari problematiche sul piano assistenziale per le condizioni di marginalità cui sono relegate. L'insediamento della popolazione è di tipo accentrato, specie nei capoluoghi, con maggiore densità di popolazione lungo le aree costiere, anche a causa delle correnti migratorie dalle aree montuose e collinari dell'interno verso i centri più grandi. La Regione è costituita da nove province che configurano

Tabella 1. Punti di debolezza dell'attuale sistema sanitario nazionale.

1. Tempi di risposta inadeguati ai bisogni dei pazienti

2. Mancanza di dialogo tra i vari attori

3. Confusione dei ruoli e dei livelli di cura tra operatori ospedalieri e territoriali

4. Gestione del paziente cronico in reparti per acuti

5. Difficoltà della totale presa in carico del paziente cronico da parte del Medico di Medicina Generale

6. Difficoltà a progettare piani delle cure personalizzati tenendo conto dei bisogni globali della persona

7. Scarso coinvolgimento del paziente e della famiglia nelle decisioni cliniche

8. Professionisti sanitari non specificatamente formati

9. Numerosità dei servizi coinvolti 
le attuali Aziende sanitarie Provinciali (ASP), per un totale di 390 comuni. La provincia più grande è $\mathrm{Pa}$ lermo che, con 1.244.680 abitanti, rappresenta circa un quarto della popolazione totale dell'isola. ${ }^{21}$

Istituita con la Legge Regionale 14 aprile $2009 \mathrm{n}$. 5, l'Azienda Sanitaria Provinciale di Agrigento è divenuta operativa a partire dal $1^{\circ}$ settembre 2009. Essa si articola in Direzione Generale, Dipartimenti, Distretti Sanitari ed Ospedalieri. Comprende 7 Distretti Sanitari (Distretto di Agrigento, Bivona, Canicattì, Casteltermini, Licata, Ribera e Sciacca) e 2 Distretti Ospedalieri, rispettivamente il Distretto Ospedaliero AG1 che comprende i presidi ospedalieri San Giovanni di Dio di Agrigento, San Giacomo d'Altopasso di Licata e Barone Lombardo di Canicattì e il Distretto Ospedaliero AG2 che comprende i presidi ospedalieri Giovanni Paolo II di Sciacca e Fratelli Parlapiano di Ribera. Complessivamente in provincia sono presenti 1.122 posti letto (di cui 977 in strutture pubbliche e 145 in private), con una media di 2,5 posti letto/1.000 abitanti, quindi al di sotto della media nazionale. ${ }^{22}$

L'ambito territoriale di riferimento dell'ASP di Agrigento, coincidente per larga parte con la Provincia Regionale di Agrigento, comprende 42 comuni, per una superficie di $3.043 \mathrm{~km}^{2}$, con una popolazione complessiva di 442.632 abitanti (al 31.12.2013) e con una densità abitativa pari a 145,45 per $\mathrm{km}^{2}$. La popolazione ultra-sessantacinquenne al 30.12.2013 ammontava in totale ad 88.341 unità, pari al $19 \%$ della popolazione complessiva. L'indice di vecchiaia è di $153,9 \%$, mentre l'indice di invecchiamento è pari al $19,9 \%{ }^{22}$

\section{Indice di vecchiaia:}

Popolazione residente $\geq 65$ anni

Popolazione residente $\leq 14$ anni

Indice di invecchiamento:

Popolazione residente $\geq 65$ anni $\quad \times 100$

Popolazione residente

L'Ospedale di Canicattì è dotato di 126 posti accreditati di cui 94 per acuti, 21 per day hospital e 32 per post - acuti (lungodegenza) (questi ultimi in atto, per problemi inerenti a carenza di risorse umane, ridotti a quattro all'interno della UO di Medicina). Esso assorbe un bacino di utenza di 86.279 (pari al $19 \%$ dell'intera provincia), affluendo al suddetto ospedale, oltre Canicattì con i suoi 40.000 abitanti, più di una decina di comuni viciniori (Castrofilippo, Naro, Camastra, Campobello di Licata, Ravanusa, Delia, Serradifalco, Montedoro, Grotte, Racalmuto, Sommatino).

Gli obiettivi della UO di Medicina dell'Ospedale di Canicattì sono coincidenti con quelli delle medi- cine interne in generale e possono essere così specificati: i) garantire l'appropriatezza del ricovero ospedaliero per trattare condizioni patologiche che richiedono prestazioni diagnostiche e terapeutiche indifferibili, urgenti o programmabili, non gestibili in ambito ambulatoriale e/o domiciliare, con modalità assistenziali le più idonee, strutturate per livelli di intensità assistenziale: ricovero ordinario (ADM Medicina Acuti - Lungodegenza), ricovero in Day Hospital; ii) assicurare una dimissione protetta nel rispetto della disabilità, della dipendenza e dei bisogni del paziente; iii) garantire le prestazioni ambulatoriali per le principali patologie di ambito internistico con una offerta di prestazioni a CUP che rispetti i criteri di equità e affidabilità, attraverso la valorizzazione delle competenze specialistiche dei suoi professionisti, nei limiti delle risorse disponibili; iv) implementare i percorsi diagnostico-terapeutici assistenziali (PDTA) Ospedale-Territorio, attivi per tre patologie croniche di area medica (Scompenso Cardiaco/Diabete mellito/BPCO).

\section{Dimissioni protette}

È prevista l'attivazione di dimissione protetta, erogabile, in pazienti con disabilità e bisogno assistenziale elevato, a domicilio, tramite ADI (assistenza domiciliare integrata), laddove i familiari siano disponibili e le condizioni abitative lo permettano, o, qualora queste condizioni non sussistano, attraverso residenzialità c/o RSA, adeguatamente dotate di personale sanitario e parasanitario. ${ }^{23}$

\section{Dimissione in RSA (Residenza Sanitaria Assistita)}

Le dimissioni protette in struttura residenziale riguardano quei pazienti fragili che, pur avendo superato la fase acuta e post-acuta della malattia durante il ricovero ospedaliero, presentano ancora bisogni assistenziali complessi e di più professionisti (controllo medico periodico, nursing infermieristico e/o trattamento riabilitativo) che rendono particolarmente problematica la gestione domiciliare.

L'RSA accoglie pazienti non autosufficienti, non assistibili a domicilio, stabilizzati sul piano clinico ma bisognevoli di cure medico - infermieristiche nelle 24 ore e di un monitoraggio dello stato di salute. È prerequisito all'ingresso la presa in carico del paziente da parte del Distretto con la progettazione, mediante valutazione multidimensionale, di un Piano di Assistenza Individualizzato (PAI) e di un percorso successivo al ricovero che dovrà essere stato condiviso con la famiglia. L'accesso alle RSA avviene esclusivamente a seguito di una valutazione multidimensionale (UVM), utilizzando lo strumento della scheda di valutazione multidimensionale SVAMA (allegato 2 del decreto 12 novembre 2007). ${ }^{24}$ La valu- 
tazione avviene in ambito ospedaliero trattandosi di paziente ricoverato e con programma di dimissione (dimissione protetta). In ogni caso la valutazione è corredata da un programma delle attività e delle prestazioni che dovranno essere attuate all'interno della RSA. Il programma assistenziale predisposto contiene l'indicazione dei tempi di permanenza in RSA, che comunque non possono superare i 12 mesi consecutivi e di norma non possono essere inferiori a giorni 30. Il Distretto Sanitario procede conseguentemente all'emissione dell'impegnativa per l'inserimento del soggetto in RSA tenendo conto dell'eventuale preferenza espressa. Il distretto di appartenenza tempestivamente informa il reparto ospedaliero dell'avvenuta emissione dell'impegnativa, dandone opportuna comunicazione al Medico di Medicina Generale (MMG) dell'assistito. In tali casi, entro i primi 30 giorni dall'inserimento, vengono effettuate verifiche da parte dell'equipe territoriale (UVM) sull'andamento del programma delle attività e delle prestazioni. La dimissione viene effettuata dal medico responsabile della RSA, coerentemente alle indicazioni della UVM. Qualora si rendesse necessario prolungare il ricovero oltre i tempi previsti, la proposta di prosecuzione deve essere inviata dal medico responsabile della struttura residenziale all'UVM territoriale, almeno quindici giorni prima della scadenza. Tale richiesta deve essere corredata da un aggiornamento della valutazione del paziente e da una riformulazione del PAI. Su tale richiesta l'UVM è tenuta ad esprimersi almeno tre giorni prima dalla data di scadenza del termine del piano di assistenza. In ogni caso dovranno essere previste soluzioni assistenziali appropriate al fine di garantire una continuità del percorso di cura del soggetto in dimissione. L'UVM del distretto di residenza dell'assistito, integrata dal MMG/PLS che procede alla valutazione multidimensionale, provvede all'elaborazione del Piano Assistenziale individualizzato (PAI). Il PAI contiene: i) Il programma delle attività e delle prestazioni che dovranno essere attuate all'interno della RSA; ii) La terapia farmacologica; iii) I presidi e gli ausili necessari; iv) I tempi di permanenza in RSA (massimo 12 mesi consecutivi e di norma non inferiori a 30 giorni); v) I tempi di verifica.

L'Asp 1 di Agrigento su cui ricade l'Ospedale di Canicattì, è dotata di tre RSA: una ubicata a Naro, una a Licata ed una a Casteltermini. La RSA viene individuata tenendo conto dell'eventuale preferenza espressa dal paziente e dei posti disponibili nelle strutture del territorio. Qualora la RSA individuata insista su un territorio diverso da quello del distretto sanitario di residenza dell'assistito, le verifiche successive alla prima valutazione verranno effettuate dall'UVM del distretto in cui è ubicata la struttura residenziale. L'UVM che ha effettuato la prima valutazione dovrà tempestivamente inviare copia del PAI già redatto all'UVM del distretto in cui insiste la RSA. L'autorizzazione al ricovero in RSA viene emessa dal Direttore del Distretto in cui è stata effettuata la valutazione. Le autorizzazioni successive verranno emesse dal Direttore del Distretto ove ricade le RSA. Qualora la valutazione sia avvenuta in distretto diverso da quello di appartenenza del paziente, i programmi delle attività e delle prestazioni dovranno essere tempestivamente trasmessi agli uffici distrettuali competenti per il territorio in cui lo stesso è residente. Gli uffici distrettuali daranno tempestiva informazione dell'avvenuta emissione dell'impegnativa al MMG del paziente.

\section{Le cure domiciliari}

Nella ASP di Agrigento, le modalità organizzative del processo assistenziale e di presa in carico relativo alle Cure Domiciliari, in coerenza con le linee di indirizzo nazionali e regionali $\mathrm{i}^{10}$ prevedono il coinvolgimento del sistema PUA, e, per le cure domiciliari integrate, della U.V.M. Il servizio prevede inoltre l'integrazione con le azioni ed i servizi offerti dagli Enti Locali in ambito sociale (Tabella 2).

Il programma operativo di consolidamento e sviluppo (POCS) 2013-2015 - innalzamento quali-quantitativo del livello di cure domiciliari integrate prevedeva che l'obiettivo da realizzare fosse parametrato al valore del $4 \%$ di anziani trattati in ADI (soglia prevista come valore - normale dalla Griglia LEA). ${ }^{25,26}$

In rapporto alla suddetta percentuale i potenziali utenti della provincia agrigentina (popolazione ultrasessantacinquenne) sono stati stimati in 3.808 soggetti.

Il servizio ADI, attraverso la promozione di un sistema complessivo di cure, ${ }^{25}$ recepito dai decreti assessoriali del 12.11.2007, del 2.7.2008 e dal DPRS 26 gennaio 2011, ${ }^{25}$ consiste in: i) Cure Domiciliari Prestazionali (consistono in prestazioni sanitarie occasionali o a ciclo programmato, comprese le visite programmate del Medico di Medicina Generale e pediatra di libera scelta (MMG/PLS). Esse, sono attivate dal MMG/PLS; ii) Cure Domiciliari Integrate di primo e secondo livello che ricomprendono quelle già definite $\mathrm{ADI}$. Questa tipologia di cure domiciliari è caratterizzata dalla formulazione del Piano Assistenziale Individuale (PAI), redatto in base alla valutazione globale multidimensionale ed erogate attraverso la presa in carico multidisciplinare e multiprofessionale (interventi: su 5-6 gg); iii) Cure Domiciliari Integrate di terzo livello che ricomprendono l'ospedalizzazione domiciliare. Sono caratterizzate da una risposta intensiva a bisogni di elevata complessità definita dal PAI ed erogata da un'équipe in 
possesso di specifiche competenze e formazione di cui fa parte il MMG. Richiedono interventi su $7 \mathrm{gg}$. su 7; supporto al paziente e alla famiglia.

\section{Le Cure domiciliari di tipo prestazionale occasionale o ciclico programmato}

Si intende una risposta prestazionale, professionalmente qualificata, ad un bisogno puntuale di tipo medico, infermieristico e/o riabilitativo che, anche qualora si ripeta nel tempo, non presupponga la presa in carico del paziente, né una valutazione multidimensionale e l'individuazione di un piano di cura multidisciplinare. Sono richieste dal medico, responsabile del processo di cura del paziente, e sono volte a soddisfare un bisogno sanitario semplice nei casi in cui il paziente presenti limitazioni funzionali che rendono impossibile l'accesso ai servizi ambulatoriali.

\section{Cure domiciliari integrate di primo e secondo livello}

Sono ricompresi in questa tipologia assistenziale interventi professionali rivolti ai malati e alle loro famiglie previsti dal PAI. L'attivazione dell'assistenza richiesta dal medico responsabile delle cure del paziente prevede il coinvolgimento del MMG nella definizione degli obiettivi e dei processi assistenziali attraverso la valutazione multidimensionale.

Si rivolge a malati che, pur non presentando criticità specifiche o sintomi particolarmente complessi, hanno bisogno di continuità assistenziale ed inter- venti programmati che si articolano sui 5 giorni (I livello) o 6 giorni (II livello). Le cure integrate di primo e secondo livello prevedono un ruolo di centralità del MMG che assume la responsabilità clinica condivisa dei processi di cura.

\section{Cure domiciliari integrate di terzo livello}

Dette cure consistono in interventi professionali rivolti a malati che presentano dei bisogni con un elevato livello di complessità in presenza di criticità specifiche: i) pazienti oncologici (e non) con malattia in fase avanzata ma non eleggibili per la fruizione delle cure palliative; ii) malati portatori di malattie neurologiche degenerative/progressive in fase avanzata (SLA, distrofia muscolare, ecc.); iii) fasi avanzate e complicate di malattie croniche; iv) pazienti con necessità di nutrizione artificiale parenterale; v) pazienti con necessità di supporto ventilatorio invasivo; vi) pazienti in stato vegetativo e stato di minima coscienza.

A determinare la criticità e l'elevata intensità assistenziale concorrono l'instabilità clinica, la presenza di sintomi di difficile controllo, la necessità di un particolare supporto alla famiglia e/o al caregiver. Presupposti di base rimangono la valutazione multidimensionale, la presa in carico del paziente e l'individuazione di un piano di cura con intervento di tipo multidisciplinare. Tali cure sono richieste dal medico responsabile delle cure del paziente. Si tratta di interventi programmati sui 7 giorni settimanali

Tabella 2. Strutture organizzative, funzioni e strumenti nel modello di funzionamento del processo assistenziale.

\begin{tabular}{|c|c|c|c|}
\hline Fase & Struttura organizzativa & Funzioni strumenti & Strumenti \\
\hline 1 & $\begin{array}{l}\text { PUA: } \\
\text { Front Office } \\
\text { Back Office }\end{array}$ & $\begin{array}{l}\text { Accoglienza e decodifica della domanda, } \\
\text { Caricamento sul portale PUA } \\
\text { Invio on line della domanda alla UVM } \\
\text { Distrettuale }\end{array}$ & $\begin{array}{l}\text { Moduli richiesta ADI } \\
\text { Portale online con programma dedicato }\end{array}$ \\
\hline 2 & $\begin{array}{l}\text { UVM: } \\
\text { CURE DOMICILIARI INTEGRATE }\end{array}$ & $\begin{array}{l}\text { Elaborazione del Progetto personalizzato } \\
\text { con definizione di profilo di cura, modalità } \\
\text { erogative, durata e tempi previsti per } \\
\text { realizzare il PAI con il coinvolgimento } \\
\text { attivo del MMG e PLS }\end{array}$ & $\begin{array}{l}\text { Schede di Valutazione Multidimensionale, } \\
\text { Verbale di sintesi del progetto individuale } \\
\text { Caricamento sul portale della Svama e degli } \\
\text { indicatori per il flusso SIAD }\end{array}$ \\
\hline
\end{tabular}

UVM:

CURE DOMICILIARI INTEGRATE
1. Trasmissione all'erogatore del programma di intervento proposto dal MMG /PLS per le Cure Domiciliari Prestazionali

2. Elaborazione e trasmissione del PAI per le

Cure Domiciliari Integrate

3. Prescrizioni: specialistiche farmaci e presidi-ausili (tramite allegato $b$ )

\begin{tabular}{llll}
\hline SOGGETTO AGGIUDICATARIO & $\begin{array}{l}\text { Erogazione delle prestazioni all'assistito } \\
\text { ESTERNO }\end{array}$ & $\begin{array}{l}\text { 1. Ricezione on line del Programma di } \\
\text { Cure Domiciliari Prestazionali e secondo il Programma di Intervento per le Intervento e/o del PAI }\end{array}$ & 2. Scheda di avvenuta presa in carico \\
(contenente data, nome cognome e & il PAI per le Cure Domiciliari Integrate & qualifica dell'operatore o degli operatori)
\end{tabular}

\author{
1. Redazione del PAI (assistenza: farmaceutica, \\ protesica, assistenza specialistica) \\ 2. invio del PAI on line all'erogatore
}


per le quali è prevista la pronta disponibilità sulle 24 ore.

\section{Rete delle cure palliative}

A far data dal $1^{\circ}$ agosto del 2015 l'ASP di Agrigento ha attivato un servizio di assistenza per le cure palliative domiciliari destinate ai pazienti oncologici in fase terminale. Prestazioni sanitarie di tipo medico, infermieristico e riabilitativo, oltreché l'eventuale supporto psicologico ai malati, vengono erogati dagli operatori professionali appartenenti alle Onlus Samo, Samot e Sisifo, in stretta sinergia con i Distretti sanitari di base e l'Unità Operativa cure palliative ed Hospice. La segnalazione per attivare il percorso di assistenza può essere effettuata dagli utenti, dai loro familiari, dai medici di Medicina Generale, dal medico ospedaliero, dai servizi sociali e di volontariato. La stessa segnalazione, unitamente ad una scheda clinica redatta dal medico di famiglia, va poi inoltrata presso i PUA del Distretto sanitario di residenza del paziente. ${ }^{27}$

Le Cure Palliative domiciliari si articolano in: i) Cure Palliative di Base (garantiscono un approccio palliativo attraverso l'ottimale controllo dei sintomi ed una adeguata comunicazione con il malato e la famiglia. Queste cure sono erogate da medici ed infermieri con una buona conoscenza di base delle cure palliative); ii) Cure Palliative Specialistiche (garantiscono interventi di equipe specialistiche multiprofessionali dedicate, per pazienti con bisogni complessi, per i quali gli interventi di base sono inadeguati. Richiedono un elevato livello di competenza e modalità di lavoro interdisciplinare).

\section{Le dimissioni facilitate}

Le dimissioni facilitate rappresentano una delle modalità più rilevanti tramite cui prende corpo la effettiva integrazione tra ospedale e territorio. ${ }^{28}$

Le facilitate, a differenze delle dimissioni protette, riguardano tutti i pazienti cronici dimessi dall'ospedale, anche quelli che godono di piena autosufficienza, e sono finalizzate ad agganciare tali pazienti alla rete territoriale allo scopo di scongiurare uno dei fattori più critici della cronicità, ossia il dropout, la perdita del paziente cronico.

In tal senso è attiva nel nostro Ospedale la dimissione facilitata per tre patologie (diabete mellito, scompenso cardiaco e BPCO): essa consiste nella segnalazione online, al momento della dimissione, del paziente al Punto Unico di Accesso (PUA) del Distretto di appartenenza, al fine di garantirne la presa in carico territoriale. $\mathrm{Al}$ momento della dimissione del paziente, infatti, lo specialista ospedaliero compila la scheda di dimissione facilitata con il piano di follow-up e lo invia per via telematica allo Sportello del paziente cronico presso il PTA. L'operatore dello Sportello del paziente cronico provvede attraverso il CUP alla prenotazione della visita. Il sistema informativo esegue l'invio di notifiche di evento ed eventuale prenotazione visita congiunta verso il MMG, di sola notifica di visita congiunta verso lo specialista e l'ambulatorio infermieristico.

Come è del tutto ovvio la dimissione facilitata persegue il suo scopo solo se è adeguatamente supportata da una efficiente ed efficace organizzazione in grado di gestire la cronicità che non può prescindere dall'integrazione tra MMG, specialisti, ospedalieri e territoriali, ed infermieri distrettuali che operano in ambulatori dedicati con follow-up attivo, e, per i pazienti più complessi, negli Ambulatori di gestione integrata e nel Day Service Ambulatoriale Territoriale.

L'ASP di Agrigento ha in funzione, in tutti i sette Distretti, un ambulatorio specialistico dedicato allo scompenso cardiaco, uno per il diabete mellito ed uno per la BPCO. Ha realizzato 4 Ambulatori a gestione integrata (AGI), di cui uno per lo scompenso cardiaco nel distretto di Canicattì ed uno a Sciacca e due per il diabete mellito, uno ad Agrigento ed uno a Licata. Ha istituito, inoltre, un ambulatorio infermieristico in ciascuno dei sette Distretti. ${ }^{28}$

Inoltre, la Medicina canicattinese conta, al suo interno, di una intensa attività ambulatoriale svolta quotidianamente, in grado di garantire una precoce rivalutazione e presa in carico del paziente in postdimissione, cosa che, da un lato permette una dimissione più precoce, dall'altro una certa serenità $\mathrm{e}$ sicurezza da parte del paziente e dei suoi familiari e il non provare quel senso di abbandono e di solitudine una volta fatto ritorno al proprio domicilio.

\section{La realtà piemontese}

\section{II contesto di riferimento: il territorio della ASL di Vercelli}

L'ASL VC di Vercelli, situata nel Piemonte Nord Orientale, comprende 91 Comuni ed è costituita da un unico Distretto Sanitario, ma si evidenziano due aree territoriali diverse per caratteristiche geografiche: l'area territoriale del Vercellese, pianeggiante, situata a sud con 44 Comuni, fra cui Vercelli, il centro urbano di maggiori dimensioni e l'area territoriale della Valsesia, montana, situata a nord.

Nella ASL VC, gli ultrasessantacinquenni risultano il 27,2\% (in Regione Piemonte risultano il $25,3 \%$ della popolazione piemontese), con una percentuale di veri anziani (75-84enni) e di grandi anziani (ultra 85 enni) pari al $14,8 \%$ della popolazione totale rispetto al $13,3 \%$ regionale.

L'indice di vecchiaia rappresenta un indicatore 
dinamico che stima il grado di invecchiamento della popolazione; valori superiori a 100 indicano una maggiore presenza di anziani ai giovanissimi. Nella ASL di Vercelli nel 2017 è risultato pari a 239,53\% rispetto al 201,34\% della Regione Piemonte, mentre l'indice di invecchiamento, che riflette il peso relativo della popolazione anziana della ASL, è risultato pari a $27,25 \%$ rispetto a $25,28 \%$ della Regione. ${ }^{29}$

La Regione Piemonte ha recepito i contenuti del PNC (piano nazionale cronicità) tramite la redazione ed implementazione di un documento recante Linee di indirizzo 2018-2019 per la presa in carico della cronicità/fragilità (Delibera 306/2018); un'altra delle direttrici strategiche nell'ambito della cronicità, è caratterizzata dal Piano di Assistenza Territoriale (P.A.T) redatto ogni 3 anni. ${ }^{30}$

Il piano aziendale della cronicità dell'ASL VC (PAC), ${ }^{31}$ parte dal presupposto che la sfida alla cronicità è una sfida di sistema, in cui occorre superare i confini tra servizi sanitari e sociali, promuovendo l'integrazione tra differenti professionalità tenendo in considerazione le problematiche di età avanzata, patologie croniche, multimorbidità $\mathrm{o}$ fragilità e prevenzione.

In relazione alle finalità descritte, la matrice del piano si è articolata in fasi sequenziali a carattere multiprofessionale e interdisciplinare quali: i) identificazione della coorte di soggetti fragili (target di riferimento); ii) stratificazione in livelli di fragilità crescenti della coorte selezionata secondo determinanti selezionate (numero di accessi al DEA, presenza di esenzione/i per patologia, ADI/ADP, consumo di farmaci classi ATC); iii) definizione delle modalità di attivazione e dei setting di erogazione della presa in carico: PAI (piano assistenziale individuale) per il paziente fragile, PDTA per il paziente cronico; iv) ridefinizione dei servizi anche tramite la riorganizzazione dell'offerta ambulatoriale e la pianificazione degli accessi al S.U.S.S. (Sportello Unico Socio-Sanitario) e al CUP; v) implementazione di un registro aziendale della fragilità (anagrafe della fragilità sociosanitaria); vi) condiviso con assistenti sociali e MMG.

L'obiettivo strategico aziendale che si intende raggiungere e perseguire è quello di eliminare/ridurre i fattori predisponenti l'aggravamento delle condizioni cliniche e, in ultima analisi, l'incremento nell' utilizzo di risorse sanitarie a più elevata intensità. In particolare, le leve strategiche utilizzate hanno interessato prioritariamente l'introduzione di innovazioni della componente organizzativa e di quella professionale attraverso i seguenti moduli sperimentali: i) inserimento della figura professionale dell'IFeC (Infermiere di Famiglia e Comunità) con il ruolo di case manager del paziente cronicofragile all'interno delle Case della Salute; ii) ridefi- nizione strutturata ed organica in team multiprofessionali e multidisciplinari di tutti gli attori in prima linea (MMG, PLS, specialisti ospedalieri e territoriali) nella gestione del paziente fragile; iii) dare rilevanza alle attività delle Case della Salute quale contesto funzionale ed operativo dove, previa identificazione del clinical manager, erogare servizi a bassa/media intensità di cure ed offrire interventi di prevenzione e promozione della salute; le CdS rappresentano anche il setting privilegiato di valutazione dell'effectiveness delle attività degli IFeC; iv) predisposizione di PDTA interaziendali orientati al paziente cronico (una patologia prevalente indipendentemente dalla presenza o meno di altre comorbidità); v) elaborazione di un modello standard di PAI (piano di assistenza individuale) per il paziente polipatologico perfezionamento di una scheda di valutazione multidimensionale (socio-sanitaria) che consenta di indagare schematicamente, le aree tematiche fondamentali, o dimensioni, che configurano la natura multipla della valutazione di un soggetto fragile: salute fisica, stato cognitivo (o salute mentale), stato funzionale, condizione economica e condizione sociale.

La redazione del documento, inoltre, ha previsto la trattazione distinta sulla gestione del paziente cronico e di quello fragile, attribuendo nel primo caso una modalità di presa in carico orientata ad una patologia prevalente (attraverso PDTA); nel secondo caso la polipatologia e le differenti variabili che concorrono a determinare la fragilità devono orientare ad una presa in carico individualizzata, tramite PAI.

\section{Distretto: il modello attuale (as is)}

L'ASL VC persegue il costante monitoraggio delle attività in ambito ospedaliero e territoriale, mediante indicatori di Performance (struttura, processo, esito) descritti nel Piano della Performance a valenza triennale ed aggiornamento annuale.

Tra le attività gestite e organizzate dal distretto troviamo le attività di case management.

\section{Case management: pianificazione dell'attività}

Inserimento del paziente in un percorso di cura che può avvenire secondo diverse modalità:

- Modalità Indirette: i) Contatto mediante interlocutori privilegiati (MMG); ii) Distribuzione di apposito materiale informativo (poster/brochure); iii) Contatto formale dell'ASL (telephone call/lettera/ SMS).

- Modalità Dirette: i) Accesso al S.U.S.S.; ii) Accesso diretto ambulatori dedicati; iii) Accesso diretto Distretto (UVG/UMVD); iv) Segnalazione Cure Domiciliari; v) IFeC.

L'inserimento del paziente all'interno di un per- 
corso di cura specifico (PDTA nel caso di paziente monopatologico e PAI in presenza di polipatologia, avverrà tramite gestionale informatico dedicato.

\section{Gestione PDTA/PAI}

Il Piano Assistenziale Individualizzato corrisponde alla sequenza delle prestazioni indicate per le patologie del paziente prescritte dal MMG /Specialista e coordinate dal case manager.

L'arruolamento del paziente si articola in una prima visita e nella definizione di un piano assistenziale contenuto nel sistema informativo. Le successive valutazioni consentono al MMG di modificare e aggiornare il piano inizialmente predefinito. Al termine di questa fase si accede alla visualizzazione del percorso di cura del paziente, programmato secondo specifiche attività mediante pianificazione $\mathrm{e}$ prenotazione delle prestazioni tramite un Centro Servizi.

\section{Verifica/rimodulazione percorso}

Il sistema consente al case manager del paziente di verificare costantemente lo stato di avanzamento del percorso al fine di individuare criticità nell'erogazione delle prestazioni, eventuali problematiche cliniche, assistenziali e organizzative. I MMG/PLS disporranno di una cartella clinica informatizzata fornita dall'ASL che, oltre alle funzionalità a supporto delle attività del Medico, è integrata con il resto del sistema al fine di consentire la gestione delle informazioni/ procedure relative ai PAI ed ai PDTA e di ricevere notifiche, quali ad esempio inserimento di un proprio assistito in un percorso da parte dello specialista oppure la modifica, chiusura, interruzione di un percorso, oppure la richiesta prescrizioni.

In Ospedale di Vercelli sono attivi i PDTA di: Scompenso cardiaco, BPCO, IRC, diabete mellito, autismo, centro disturbi cognitivi e demenze.

\section{Integrazione Ospedale Territorio (O-T)}

La dimissione dall'ospedale non deve più essere esclusivamente l'atto conclusivo del ricovero, ma l'elemento di congiunzione nel percorso assistenziale del paziente dove l'Ospedale ed il Distretto si integrano per valutare il reale bisogno del paziente.

Fin dalle prime fasi del ricovero in Ospedale, si procede ad una pianificazione della dimissione con l'individuazione del setting assistenziale adeguato ed appropriato in termini clinici ed organizzativi, in modo da evidenziare da subito i casi che potenzialmente necessiteranno di continuità assistenziale, in previsione di progetti sia residenziali che domiciliari.

I setting di cura e assistenza condivisi ed elaborati possono prevedere: i) Attivazione NOCC/NDCC; ii) Inserimento in CAVS; iii) Attivazione ADI/ SID: ser- vizio distrettuale cure domiciliari; iv) Attivazione SUSS e Commissioni Multidimensionali (UVG/ UMVD) per progetti Sociosanitari; v) attivazione di servizio di cure palliative Hospice (10 p.l.), AMAP (6 p.1.).

\section{Nucleo ospedaliero di continuità assistenziale e nucleo distrettuale di continuità assistenziale (N.O.C.C./N.D.C.C)}

L'ASL VC in attuazione al modello previsto dalla DGR n. 27-3628 del 28.3.2012 e DGR 6-5519 del 14 marzo 2013 si è dotata di 1 Nucleo Ospedaliero di Continuità delle Cure (N.O.C.C.) e di 1 Nucleo Distrettuale Continuità delle Cure (N.D.C.C).

I due presidi ospedalieri sono dotati di 215 P.L. presso il Presidio Ospedaliero ( P.O.) di Vercelli e 82 P.O. a Borgosesia.

Non sono presenti posti letto di lungodegenza e continuità assistenziale.

Il N.O.C.C, operativo presso entrambi i Presidi dell'ASL VC, è composto da: i) Infermiere; ii) Assistente Sociale.

Il N.O.C.C si avvale dell'apporto clinico competente in base al progetto individuale del paziente avviando: i) funzioni di coordinamento trasversale dei servizi ospedalieri e dei dipartimenti assistenziali con le strutture assistenziali (CAVS) e territoriali aziendali (Cure Domiciliari) ed extraaziendali coinvolte nel processo di cura; ii) Attività di promozione delle iniziative di informazione e formazione rivolte a tutti gli operatori coinvolti nel progetto/percorso e finalizzate alla conoscenza dei criteri di individuazione dei pazienti per i quali attivare il Nucleo; iii) concertazione della metodologia e dei tempi del processo di presa in carico nonché gli strumenti di comunicazione/monitoraggio.

Gli N.D.C.C. delle sedi di Vercelli e Borgosesia operativi presso il Distretto dell'ASL VC sono composti da: i) Dirigente Medico del Distretto; ii) Coordinatore delle Cure Domiciliari, CPSE infermieristico; iii) Infermiere; iv) Assistente Sociale di competenza territoriale del paziente.

Il N.D.C.C. coinvolge e informa tempestivamente di volta in volta il MMG o il PLS dell'assistito e i servizi territoriali, sociali ed eventualmente i servizi specialistici. E riferisce al Direttore del Distretto.

Il N.D.C.C. svolge la funzione di individuazione, in collaborazione con il NOCC e con la rete familiare o altre figure di supporto dell'assistito, del setting appropriato per il percorso postdimissioni coerente con $\mathrm{i}$ bisogni individuati.

IL N.O.C.C. e il N.D.C.C. utilizzano a fini statistici il sistema informativo regionale (WEB-anziani) e formalizzano informaticamente tutte le fasi del percorso assistenziale del paziente, in modo da garantire le informazioni cliniche e terapeutiche necessarie ai 
vari punti della rete, nonché la tracciabilità in tempo reale dei passaggi. É prevista attivazione diretta del N.O.C.C. e N.D.C.C. anche per pazienti in DEA sia durante l'accesso che in O.B.I.

\section{CAVS}

L'Asl di Vercelli ha provveduto alla attuazione della funzione extraospedaliera di continuità assistenziale a valenza sanitaria individuando 40 posti letto di CAVS, di cui 18 nell'Ospedale di Vercelli e 18 nell'Ospedale di Borgosesia. Tali posti letto, come precisato dalla DGR 26-1653/2015, rappresentano un setting in cui prevale la componente assistenziale rispetto alla parte clinica, e deve seguire specifici criteri di tipo clinico, assistenziale, riabilitativo e socio-ambientale. I criteri di inclusione al CAVS sono di seguito riportati: i) superamento della fase acuta della patologia; ii) stabilità clinicaassistenziale-comportamentale; iii) assenza di indicazioni a setting riabilitativo individuale specifico; iv) paziente con un progetto riabilitativo non immediatamente attivabile per condizioni cliniche e socio-assistenziali; v) presenza di condizioni cliniche tali da non permettere al paziente di seguire un appropriato percorso di continuità di cure al domicilio per motivi socio-familiari, forniture di ausili, attesa di un luogo di cura adatto, conclusione di un percorso terapeutico semplice, addestramento del care giver.

\section{Centrale Operativa delle Cure Domiciliari e della Continuità assistenziale}

La Centrale operativa delle Cure domiciliari e della Continuità Assistenziale è dotata di personale infermieristico dedicato ed è attiva 5 giorni alla settimana. Nel corso del 2018 è stata attivata su tutto il territorio anche la procedura di presa in carico tramite portale del paziente, con l'utilizzo del software dedicato.

Tutti i MMG/ PLS sono stati coinvolti, e formati, parimenti a tutto il personale delle Cure Domiciliari e degli uffici di supporto e viene utilizzata piattaforma informatica.

La centrale operativa riceve tutte le richieste di attivazione di interventi domiciliari e continuità assistenziale svolgendo funzione di supporto e collegamento e coordinando le prestazioni sanitarie e gli interventi domiciliari.

La continuità assistenziale è rivolta ai pazienti a rischio di ospedalizzazione prolungata o dimissione difficile residenti nel distretto di competenza.

Il progetto di continuità dall'Ospedale verso il Distretto può realizzarsi mediante: i) dimissione ordinaria con eventuale educazione/istruzione del paziente e del care giver; ii) attivazione cure domiciliari con o senza prescrizione di ausili; iii) attivazione posti di continuità assistenziale (CAVS); iv) attivazione Cure Palliative; v) attivazione del servizio sociale territoriale; vi) ricovero in strutture di riabilitazione/lungodegenza.

\section{Assistenza domiciliare integrata (A.D.I.)}

Assicura alle persone affette da patologie gravi, in condizioni di non autosufficienza o malati terminali, cure sanitarie presso il proprio domicilio sotto la responsabilità del medico di famiglia e in presenza di caregiver validi. Comprende visite del medico di Medicina Generale e dei medici specialistici, prestazioni infermieristiche, riabilitative e di assistenza alla persona. La proposta di attivazione viene formulata dal medico di famiglia.

\section{Assistenza domiciliare integrata con l'Unità Operativa di Cure Palliative (A.D.I. - U.O.C.P.)}

Si tratta di una forma di Assistenza Domiciliare Integrata rivolta a persone in una fase avanzata di malattia e che perciò necessitano di cure palliative. Queste cure vengono prestate dal medico di famiglia in collaborazione con gli infermieri domiciliari, i medici specialisti della ASL e altro personale specializzato in cure palliative appartenente ad associazioni convenzionate (ad esempio fondo Edo Tempia).

\section{Assistenza domiciliare programmata (A.D.P.)}

Assicura visite programmate del medico di famiglia presso il domicilio di pazienti non deambulanti, con particolari patologie croniche o invalidanti, che non sono in grado di recarsi o di essere trasportati presso l'ambulatorio.

\section{Assistenza domiciliare infermieristica (S.I.D.)}

Si tratta di prestazioni infermieristiche (medicazioni, terapie iniettive, ecc.) rivolte a pazienti che, a causa di limitazioni fisiche e/o ambientali, non sono in grado di recarsi presso gli ambulatori infermieristici del Distretto.

\section{Prestazioni infermieristiche estemporanee (P.I.E.)}

Sono prestazioni occasionali (prelievo ematico, elettrocardiogramma) effettuate a soggetti per i quali non è prevista una presa in carico continuativa. Le prenotazioni per l'esecuzione delle prestazioni possono essere effettuate presso le sedi delle Cure Domiciliari con tempi di attivazione di norma entro 4 giorni per pazienti affetti da patologie croniche (con bisogni differibili nel tempo) ed entro 24 ore solo per bisogni urgenti (pazienti con bisogni non differibili). Tutti i servizi domiciliari sono attivati dal medico di famiglia. 


\section{Case della Salute}

L'ASL VC, seguendo le Linee di Indirizzo regionali che recepiscono il PNC, ha da tempo intrapreso una riorganizzazione dei servizi territoriali che individua nella Casa della Salute (D.G.R. n. 26-1653 del 29.6.2015) il setting ideale per attuare una gestione integrata delle patologie croniche. Esse costituiscono idealmente il primo punto di accesso ove l'utenza può trovare una prima risposta al proprio bisogno socio-sanitario. Nell'ASL VC sono presenti 5 Case della Salute (Santhià, Gattinara, Varallo, Coggiola, Cigliano), 3 poliambulatori e 28 punti prelievo. All'interno delle Case della Salute è presente personale medico, infermieristico e amministrativo che è affiancato dalla figura dell'Infermiere di Comunità e famiglia ( $\mathrm{IFeC}$ ). Attualmente quest'ultimo è assegnato al modello sperimentale $\mathrm{CdS}$ di Santhià ma si prevede una assegnazione a tutti gli ambiti territoriali. Tale figura collabora strettamente con i MMG per la presa in carico dei pazienti fragili, garantendo interventi personalizzati e di health literacy, oltre che di prevenzione primaria, operando prevalentemente al domicilio dei pazienti e delle loro famiglie, facilitando il processo di adattamento ed autogestione delle malattie croniche e della conseguente disabilità, contribuendo all'accesso al sistema in rete dei servizi territoriali di cure primarie. Durante il 2019 è stata attivata una seconda edizione del Master IFeC in collaborazione con UPO (Università del Piemonte Orientale), durante il quale verranno formati un numero di infermieri di famiglia programmato per tutte le case della salute dell'ASL. In questo modo il programma di Infermieristica di Famiglia verrà esteso a tutta la popolazione dell'ASL entro la fine del 2021.

\section{Sportello Unico Servizi Socio-Sanitari (S.U.S.S.) / Punto Unico di Accesso (PUA)}

Lo Sportello Unico Socio Sanitario (SUSS) realizzato in cooperazione con gli enti locali delle funzioni sociali rappresenta il gateway unitario di accesso dell'utente ai servizi sociosanitari dell'ASL, rivolta principalmente ai soggetti non autosufficienti ed ai loro caregiver (oppure può essere il case manager a proporre la presa in carico) siano essi anziani, minori o adulti affetti da patologie croniche o invalidanti. Esso infatti rappresenta uno snodo cruciale per la individuazione, la ricognizione, l'orientamento ed il monitoraggio della persona fragile ad ogni età.

Gli obiettivi sono: i) ridurre il disorientamento delle persone con domanda di assistenza (accoglienza); ii) favorire l'accesso alla rete integrata dei servizi socio-sanitari territoriali; iii) fornire l'informazione, l'orientamento e la gestione della domanda (informazione relativamente ad opportunità, risorse, prestazioni e possibili agevolazioni nonché sul sistema dei servizi esistenti ed accessibili); iv) garantire la presa in carico e l'attivazione della rete dei servizi integrati.

È stata attivata e condivisa tra tutti gli sportelli una piattaforma informatica finalizzata alla: i) registrazione delle richieste; ii) condivisione in rete dei dati (utenza/bisogno/risposta); iii) archiviazione ed elaborazione delle informazioni per una corretta gestione dei flussi informativi.

\section{Il percorso organizzativo del paziente fragile}

L'organizzazione dell'assistenza al paziente fragile prevede facilitazioni nel sistema attraverso punti di accesso spontanei o condizionati dalla necessità di cura e l'attribuzione per ciascun paziente di un case manager e di un clinical manager: i) case manager: è il professionista che provvede all'assegnazione e al coordinamento dei servizi socio-sanitari destinati alla gestione clinica di un determinato target di utenti. Ha la funzione di coordinamento gestionale. La letteratura internazionale sovrappone il concetto di case manager a quello di care manager educatore per il paziente e la propria famiglia ma anche per il medico e gli altri soggetti socio-sanitari; ii) clinical manager: è il medico referente, responsabile della presa in carico e cura la predisposizione e l'aggiornamento del PAI. L'arruolamento del paziente avviene attraverso la sottoscrizione del Patto di Cura e la condivisione del PAI.

Il modello sperimentale di clusterizzazione in classi di soggetti fragili dell'ASL VC ha subito alcune integrazioni/modifiche che riguardano: i) stratificazione della fragilità utilizzando tutte le esenzioni per patologia cronica, comprese le malattie rare e la condizione di invalidità; ii) definizione di un cluster di pazienti identificati in base alla presenza di una sola patologia.

Il modello di clusterizzazione è stato predisposto attraverso una matrice piramidale a fragilità crescente. L'integrazione delle informazioni assunte consente di aumentare la sensibilità dello strumento (Figura 1).

\section{La realtà del Friuli Venezia Giulia}

La regione Friuli Venezia Giulia /FVG) condivide con la Liguria la popolazione con l'età media più avanzata, 47 anni (dati ISTAT al $1^{\circ}$ gennaio 2019). Su una popolazione di 1.215.220 abitanti l'indice di vecchiaia, ovvero il rapporto percentuale fra popolazione di età maggiore a 65 anni e di età $0-14$, è di 191.8. Il problema delle cronicità e delle relazioni fra ospedale territorio è pertanto particolarmente rilevante, nonostante molte siano ancora le cose da fare. Fino alla riforma del 2014 (Legge regionale FVG 
2015, n. 26), ${ }^{32}$ l'assetto delle Aziende Sanitarie prevedeva una notevole articolazione e differenziazione: due Aziende Ospedaliero-Universitarie (Udine e Trieste), non collegate direttamente al territorio, un'Azienda Ospedaliera Pordenone, cui afferivano 2 ospedali spoke (Spilimbergo e S. Vito al Tagliamento); tre aziende con presenza di ospedali e due aziende territoriali pure (Provincia di Pordenone e di Trieste). L'intento della riforma di legge era in particolare quella di (Art. 2 comma a e b): [...] potenziare l'assistenza primaria, rendendola il più possibile vicina alle esigenze e ai bisogni dei cittadini, nonché facilmente accessibile e fruibile dai medesimi; determinare un riequilibrio delle risorse tra i settori nei quali viene espletata l'attività del Servizio sanitario regionale [...]. Il nuovo assetto pur prevedendo l'unificazione del territorio con le strutture ospedaliere ha solo soddisfatto parzialmente le aspettative. In particolare appariva debole e confuso il rapporto fra Aziende Hub di Udine e Trieste e quelle spoke. Esemplare il caso dell'AAS 2 Bassa Friulana Isontino, con due strutture ospedaliere collegate Latisana e Palmanova che erano spoke rispettivamente di Udine e Gorizia-Monfalcone (che, a loro volta, facevano riferimento a Trieste). L'attuale riforma sanitaria, entrata in vigore dal $1^{\circ}$ gennaio 2020 ha strutturato 3 aziende ospedaliere-territoriali con la presenza di ospedale di $2^{\circ}$ o $3^{\circ}$ livello e ospedali di rete. Benché questo possa favorire i rapporti tra ospedale e territorio con un'unica regia, rimangono molte problematiche sia su base normativa, quanto organizzativa e culturale. La Regione, tra l'altro, non ha ancora contestualizzato nel proprio territorio un piano regionale per la cronicità, ma la decennale quotidianità sul paziente anziano, fragile e multi-problematico hanno catalizzato lo sviluppo di approcci integrati Ospedale-territorio-sociale quale strumento efficace nella gestione del paziente anziano/fragile/isolato (testimoniato anche dalla presenza di una direzione centrale Salute, politiche sociali e disabilità). Analogamente alle realtà descritte nel presente articolo, anche nella Regione FVG sono stati progressivamente introdotti e sono attualmente consolidati i percorsi di continuità delle cure tramite lo strumento PAI e lo sviluppo delle azioni sul territorio in collaborazione con la Medicina di base, l'Infermiere di Comunità (IdC) e il Fisioterapista di Comunità $(\mathrm{FdC})$. La sinergia delle collaborazioni con il sistema sociale si realizza in particolare nel momento della dimissione dall'Ospedale (supporto nell'assistenza a domicilio, nell'addestramento dei caregiver domiciliari e nel compenso alle situazioni di disagio (ad esempio l'igiene e la fornitura pasti a domicilio) e nel contrasto dell'istituzionalizzazione (sostegno del Fondo per l'Autonomia Possibile $(\text { FAP })^{33}$ finalizzato a supportare il sostegno con personale a domicilio degli anziani/non autosufficienti. In questi percorsi il Sistema Sanitario viene chiamato a svolgere una funzione non solo di cura ma anche di formazione, di accompagnamento e di sorveglianza a domicilio, garantendo una presa in carico precoce delle situazioni e delle possibili criticità. Nei percorsi di contrasto all'istituzionalizzazione invece viene valorizzato il ruolo di valutazione delle condizioni di salute e di autonomia in collaborazione con il sistema sociale dei Comuni.

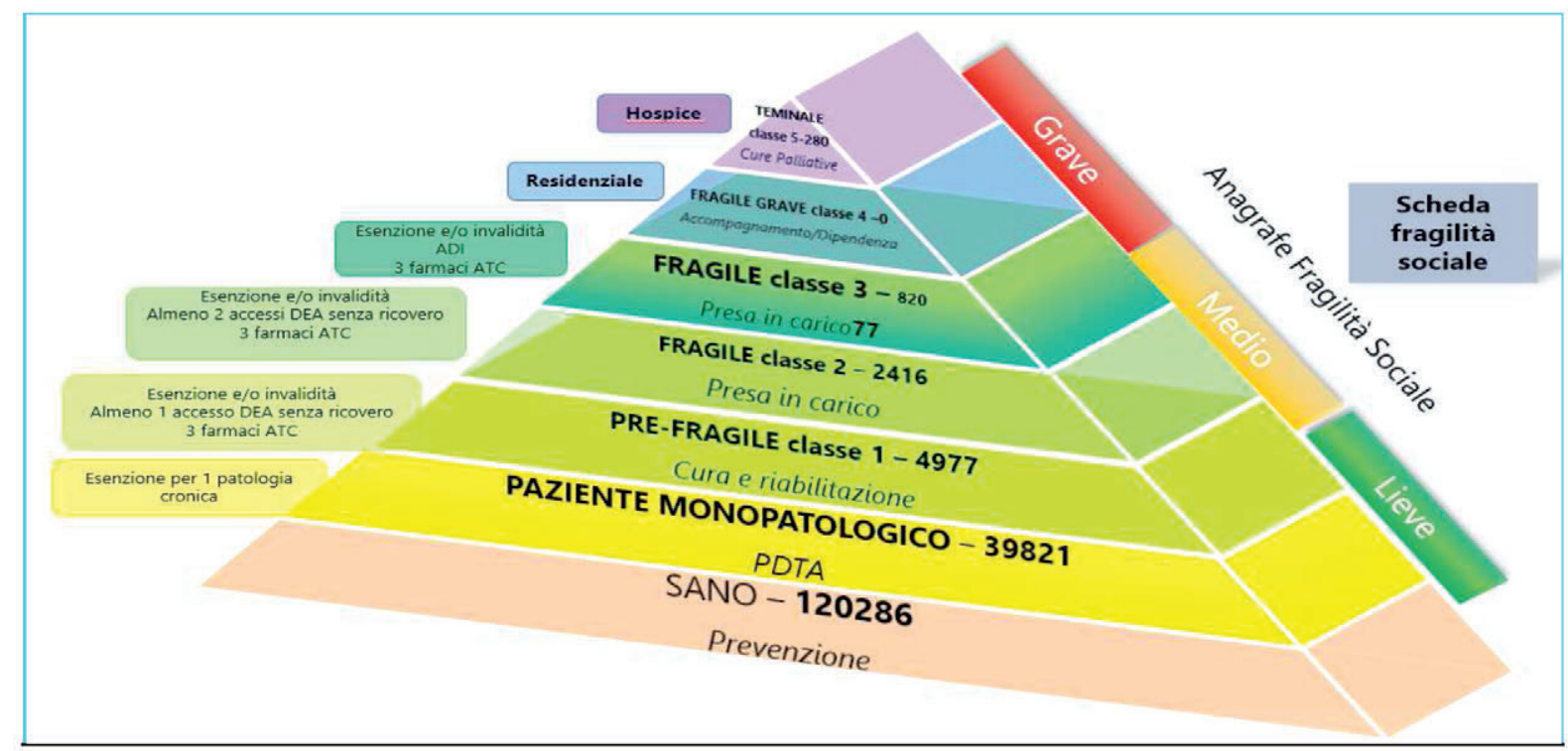

Figura 1. Scheda fragilità sociale. 
Il Distretto garantisce una continuità dell'assistenza al domicilio in collaborazione con i servizi ospedalieri e di corretta gestione delle cronicità, garantendo spazi e risorse non disponibili nelle strutture ospedaliere, votate al trattamento dell'acuzie. Una buona collaborazione tra i servizi Ospedale - territorio è la chiave dell'efficacia degli sforzi del sistema sanitario pubblico. La figura chiave degli interventi, sia in ambito sanitario che sociale, è il case manager tra gli operatori dei servizi, quale professionista in grado di cogliere e guidare il bisogno delle persone e indirizzarlo al giusto professionista della rete socio-sanitaria. Una criticità è rappresentata dalla mancanza di adeguati sistemi informatizzati in grado di facilitare la tempestiva trasmissione e condivisione delle informazioni tra i professionisti della rete. Anche in FVG, un esempio tangibile dell'integrazione socio-sanitaria è rappresentata dal Punto Unico di Accesso (PUA) dove il cittadino è in grado di trovare professionisti in grado di informalo, guidarlo e indirizzarlo correttamente nel panorama di servizi disponibili. Il momento chiave dell'integrazione Ospedale - territorio può essere riassunto nell'UVM (Unità di Valutazione Multidisciplinare) dove i professionisti sanitari potenzialmente coinvolti (ad esempio, Specialista Ospedaliero, Medico di Medicina Generale, Pediatra di Libera Scelta, Medico di Distretto, Infermiere di Continuità, Infermiere del PUA, Infermiere di Comunità, Fisioterapista Ospedaliero e di Comunità, Assistente Sociale e rappresentati del terzo settore), si incontrano per concordare con l'interessato e i familiari le modalità per favorire i percorsi di assistenza (sanitaria e sociale) dopo la dimissione. Il tutto viene trascritto in un verbale strutturato sottoscritto da tutte le parti e affidato al Case Manager che ne garantirà la realizzazione nel tempo o avvierà gli opportuni percorsi di revisione/aggiornamento. Il percorso di dimissione dei pazienti che termina con la dimissione (come concordato nell'UVD) inizia sostanzialmente poche ore dopo il ricovero (già dopo 24/48 ore viene segnalato al distretto l'elenco degli accolti e le problematiche di salute per cui sono stati ricoverati) dando in questo modo avvio al percorso costruzione dei percorsi di continuità e di attivazione dei sostegni in prospettiva della dimissione. Un ulteriore elemento di continuità tra ospedale e territorio è rappresentato dalle finalità di formazione dei caregiver domiciliari (familiari e non) che inizia sempre prima della dimissione, viene rinforzata in caso di accoglimento in $\mathrm{RSA}^{34}$ e prosegue a domicilio, sia con azioni di formazione che con finalità di sorveglianza. Un importante anello di congiunzione tra l'Ospedale e il Territorio è rappresentato dall'RSA a cui sono affidate finalità di cure intermedie sia di tipo riabilitativo che di continuità delle cure tra un setting assistenziale estensivo e il contesto domiciliare. L'RSA svolge anche funzioni di supporto al territorio, garantendo la presa in carico precoce di situazioni che non richiedono il ricovero in ospedale o anche temporanee funzioni di sollievo nelle situazioni di maggior complessità sociale (sgravando l'Ospedale in parte di ricoveri inappropriati). $\mathrm{Da}$ ricordare anche la diffusione su tutto il territorio regionale degli Hospice dedicati al supporto della terminalità. Le diverse modalità con cui si realizzano $i$ percorsi di continuità in ambito distrettuale (ADI, ADP, SID, SRD - Sistema Riabilitativo Domiciliare) vengono gestite in ambito distrettuale in collaborazione con i diversi professionisti coinvolti (MMG, PLS, palliativista, IdC, FT, ecc.) (Figura 2).

\section{La realtà di un ospedale di rete: \\ S. Daniele del Friuli (UD)}

L'ospedale di S. Daniele è dal $1^{\circ}$ gennaio 2020 ospedale Spoke dell'Azienda Sanitaria Universitaria Friuli Centrale ed è il riferimento per il distretto Collinare (Distretto 3) e Medio Friuli (Distretto 4), ovvero 112.000 abitanti su una superficie di $760 \mathrm{~km}^{2}$ in un'area rurale con presenza di attrazione dai distretti contermini (Figura 3). Il vecchio atto aziendale, ${ }^{35}$ tuttora vigente, incoraggiava la creazione di rapporti fra Ospedale e territorio. Nell'ambito di tale progettualità il reparto di Medicina Interna di S. Daniele (78 letti e 2658 ricoveri ordinari nel 2019) ha avviato dall'inizio del 2019 l'Ambulatorio delle Cronicità, un supporto ai Colleghi MMG dei due distretti di pertinenza, a loro volta strutturati in 2 Aggregazioni Funzionali Territoriali (AFT), ciascuno. L'ambulatorio offre una risposta rapida e alternativa di supporto decisionale e, nel contempo, contribuisce a contenere ricoveri e DH impropri. Nelle ore del mattino, dal lunedì al venerdì, infatti un internista (il medico responsabile del $\mathrm{DH}$ ) è disponibile per i MMG e raggiungibile per consulti a un numero telefonico riservato nella fascia oraria dalle ore 9 alle ore 12 . Eventuali approfondimenti che dovessero risultare necessari trovano prontamente risposta grazie alla possibilità di accedere ad un'offerta ambulatoriale dedicata collegata ad attività di DH laddove indicato. Un'azione sviluppata è quella di collegarlo ai pazienti in ossigenoterapia, poiché nell'area non è presente un servizio di Pneumologia. Si tratta di un'attività finalizzata a garantire la continuità e la qualità nella cura dei pazienti a domicilio e l'appropriatezza prescrittiva, inviando al centro Hub i soli casi che necessitino di approfondimenti diagnostici. Prima dell'avvio del programma è stato definito un protocollo operativo aziendale, basato sulle più recenti LG nazionali e internazionali. Il progetto è ancora in fase di avviamento. Nei prossimi mesi si avvierà una valutazione dei dati dell'attività del- 
1'Ambulatorio per le Cronicità, per valutare il reale impatto sul numero di ricoveri, sulla degenza media e la prevenzione di ricoveri ripetuti a 30 giorni dalla dimissione.

\section{Conclusioni}

Come visto, l'elemento centrale del processo di continuità assistenziale è la presa in carico del paziente dall'inizio fino al completamento del suo percorso di salute, senza alcuna soluzione di continuità nel ricevere le cure/assistenza dai vari soggetti erogatori. La presa in carico può essere definita come: processo integrato e continuativo che si configura come governo di un insieme articolato e coordinato di interventi rivolti a soddisfare un bisogno complesso. L'obiettivo è quello di superare la logica settoriale e frammentaria degli interventi attraverso l'erogazione di singole prestazioni, segmentate ed isolate, che risultano, talvolta, inefficaci ed inefficienti.

Il Distretto è il fulcro dell'articolazione territoriale cui è affidato il compito di assicurare alla popolazione residente la disponibilità, secondo criteri di equità, accessibilità ed appropriatezza, dei servizi di tipo sanitario e sociale ad alta integrazione sanitaria.

Il Distretto svolge un ruolo cruciale nella: i) rilevazione dei bisogni di salute della popolazione; ii) pianificazione e valutazione dell'offerta di servizi; iii) realizzazione delle attività di promozione della

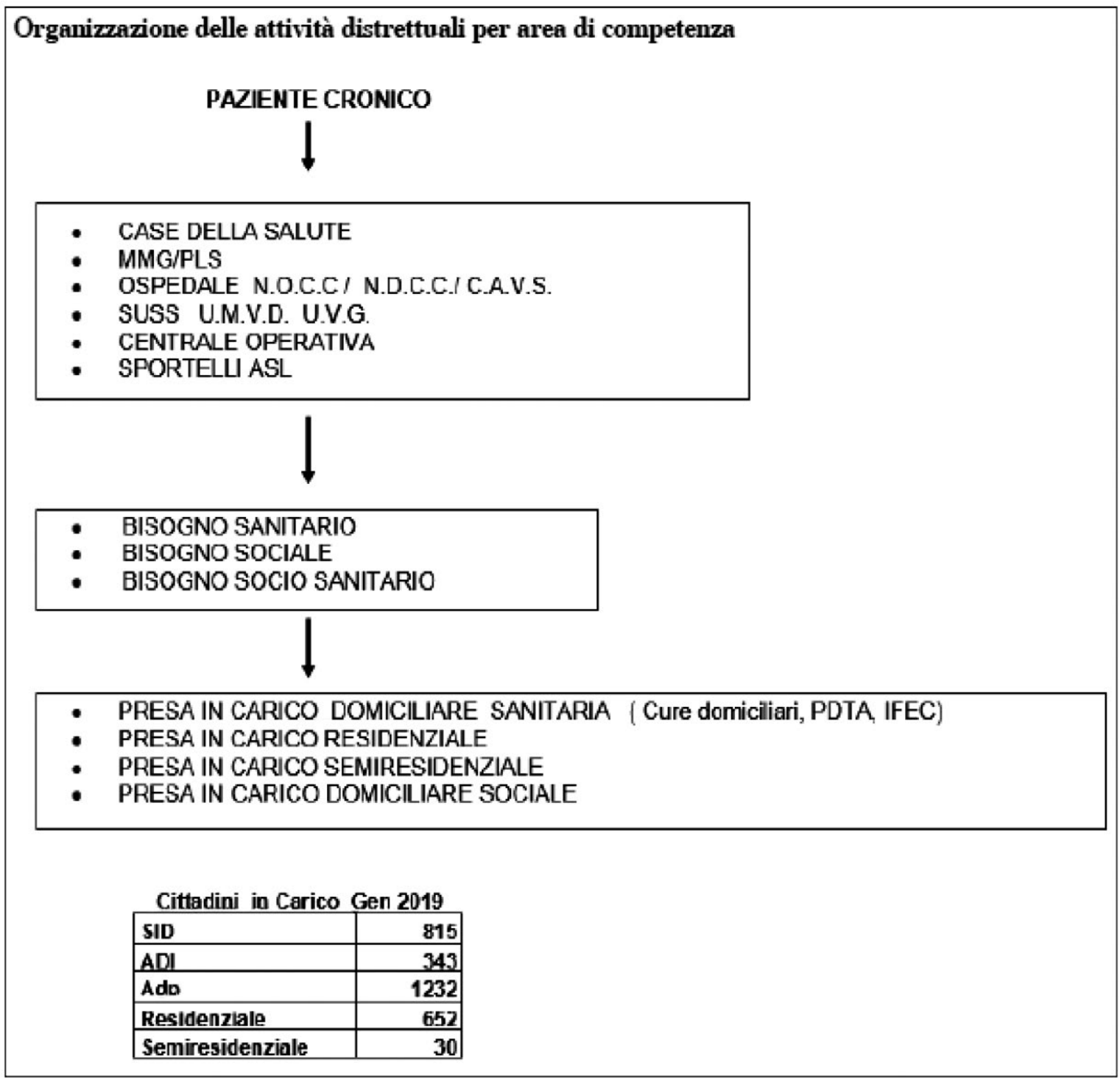

Figura 2. Organizzazione attività distrettuali per aree di competenza. 
salute; iv) prevenzione delle malattie e delle disabilità e nell'integrazione tra attività sanitarie e sociali.

La realizzazione nella realtà piemontese delle Case della Salute rappresenta un nuovo modello organizzativo di assistenza integrata rispetto al servizio sanitario nazionale, una realtà pensata per il paziente che trova il suo riferimento in un unico luogo, posto all'incrocio tra riorganizzazione sanitaria e integrazione sociosanitaria.

Al centro dell'attenzione delle Medicine Interne è certamente il paziente fragile. La definizione di fragilità richiama il concetto più spiccatamente sanitario di multimorbidità, intesa come insieme di patologie correlate che possono alternativamente $\mathrm{o}$ in maniera sinergica: ${ }^{36,37}$ i) determinare un peggioramento della qualità di vita e/o aumentare il tasso di mortalità per tutte le cause; ii) aumentare l'accesso non programmato ai servizi sanitari e/o il tasso di ospedalizzazione; iii) aumentare la necessità di polifarmacoterapia; iv) determinare un incremento degli eventi avversi, quali quelli farmaco - correlati; v) riacutizzare patologie croniche di cui il paziente è affetto.

Inoltre, dal punto di vista terapeutico, il trade off dei rischi e dei benefici dei trattamenti è spesso incerto in quanto frequentemente basato su prove di efficacia degli interventi sanitari derivanti da trial clinici che escludono il paziente con multimorbidità.

Il PDTA costituisce uno strumento di tutela delle opportunità di salute e necessita della definizione di un contesto organizzativo in cui essere implementato e adattato, e della collaborazione costante tra i vari specialisti (definendone ruoli e competenze) con finalità di tipo diagnostico-terapeutico, riabilitativo e preventivo (quali ad esempio, contenere la disabilità, posticipare l'evoluzione della malattia), integrando gli interventi sanitari con quelli sociali, qualora si riscontrino condizioni di fragilità.

Un aspetto spesso costitutivo di alcuni PDTA è dato dall'integrazione non solo funzionale (intera-

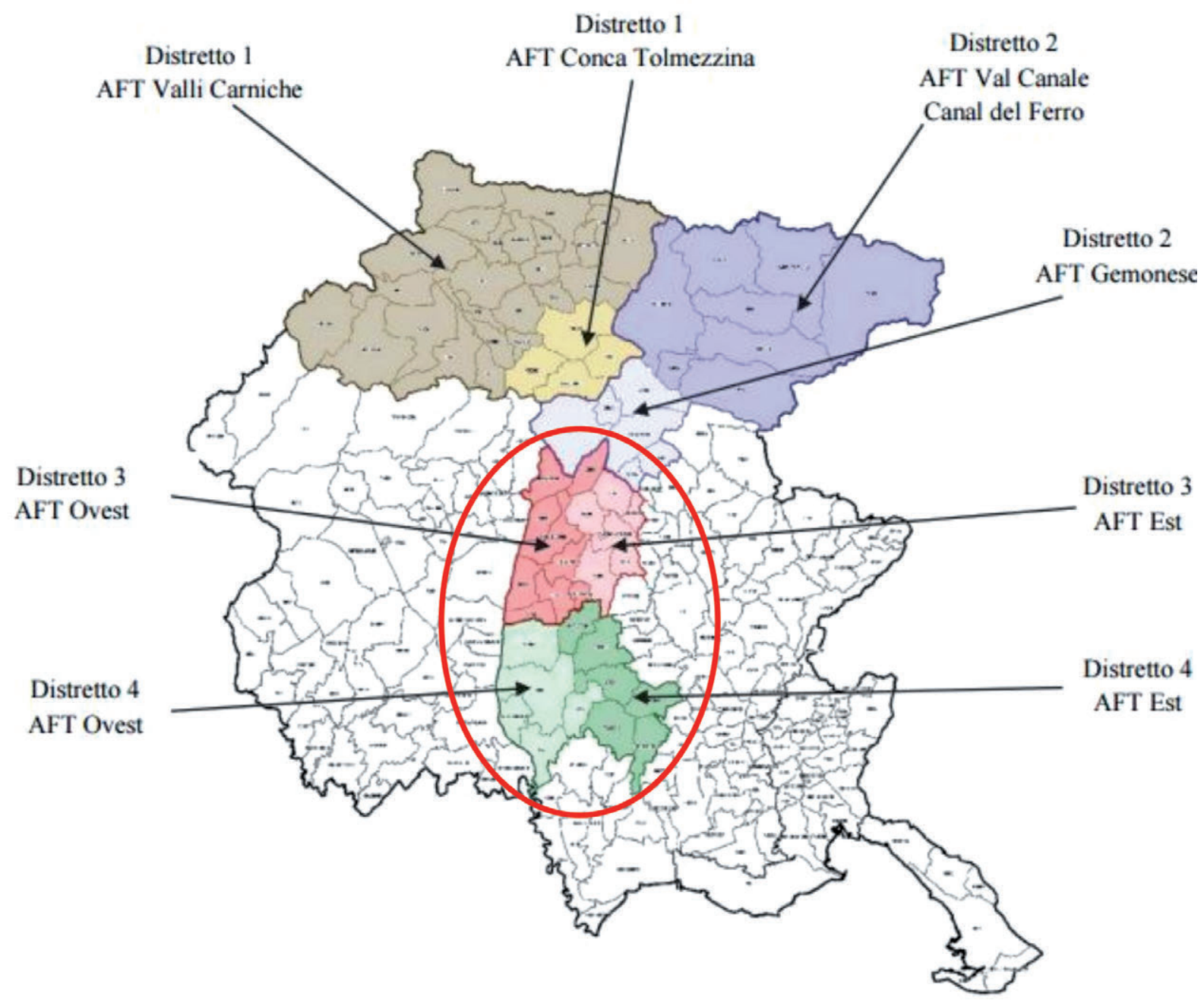

Figura 3. I Distretti afferenti all'Ospedale di S. Daniele. 
zione dei professionisti coinvolti) ma anche strutturale, rispetto ad una modalità di gestione della presa in carico che intercetta differenti setting (es., ospedale Hub e Spoke), ridefinendo compiti e responsabilità del team.

Occorre sottolineare, inoltre, che a livello semantico anche la definizione di paziente cronico è mutata in quanto a livello epidemiologico si presentano: i) cronicità ad alta complessità (patologia oncologica) in cui le innovazioni tecnologiche (sia diagnostiche che terapeutiche) hanno determinato per alcune patologie oncologiche la riduzione del tasso di mortalità con conseguente cronicizzazione della stessa patologia; ii) nuove cronicità a cui afferiscono le patologie neurologiche, reumatologiche ed immunologiche recepite anche dal Piano Nazionale Cronicità.

Nell'ambito di tale progettualità interessante l'esperienza dell'Ambulatorio delle Cronicità portata avanti nella Medicina dell'ospedale friulano: essa rappresenta uno strumento di presa in carico dei pazienti con problematiche di cronicità importanti per frequenza e difficoltà di management quali lo scompenso cardiaco, la BPCO, le anemie multi-fattoriali.

\section{Bibliografia}

1. Mauri M. Ricerca finalizzata - rapporto conclusivo Principi guida tecnici,organizzativi e gestionali per la realizzazione e gestione di Ospedali di alta tecnologia, supp. Monitor n.6, ASSR, Roma, 2003.

2. Epicentro. Il portale dell'epidemiologia per la sanità pubblica a cura dell'Istituto Superiore di Sanità. data ultima consultazione online 03/02/2020.

3. Capobianco G. L'assistenza all'anziano "fragile" in "Cambiamenti climatici, rischi per la salute e misure di prevenzione 27 -29 maggio 2013 organizzato da CNESPS - Istituto Superiore di Sanità e DG prevenzione - Ministero della Salute.

4. Bissolo G, Fazi L. Costruire l'integrazione sociosanitaria. Attori, strumenti e metodi. Roma: Ed. Carrocci; 2004.

5. Banchero A. Programmare i servizi sociali e socio sanitari. Bologna: Il Mulino; 2005.

6. Ciocia A. Per un welfare dalla parte dei cittadini: Aspetti territoriali della domanda delle politiche sociali. Roma: Carrocci; 2007.

7. Formez. Sportelli Sociali per l'accessibilità dei servizi, Rapporto di ricerca. Roma; 2005.

8. Pesaresi F. La porta sociale: le tendenze in Italia. In: Prospettive sociali e sanitarie n.18, 2008.

9. Devastato G. La porta unica di accesso. Progetto di ricerca promosso dal Ministero del Welfare dal titolo "Il sistema di protezione e cura delle persone non autosufficienti"; 2010.

10. Marengoni A. Aging with multimorbidity: a systematic review of the literature. Ageing Res Rev. 2011;10:4309.

11. Rechel B. Ageing in European Union. Lancet 2013;381:1312-22.
12. Wolinsky FD, Johnson RJ. The use of health services by older adults. J Gerontol 1991;46:345-57.

13. Gaugler J, Kane R. Family care for older adults with disabilities: Toward more targeted and interpretable research. Int J Aging Hum Dev 2002;54:205.

14. Tjia J, Velten SJ, Parsons C, et al. Studies to reduce unnecessary medication use in frail older adults: a systematic review. Drugs Aging 2013;30:285-307.

15. Pawlson LG. Hospital length of stay of frail elderly patients: primary care by general internists versus geriatricians. J Am Geriatr Soc 1988;36:202-8.

16. Milte, R. and M. Crotty, Musculoskeletal health, frailty and functional decline. Best Pract Res Clin Rheumatol 2014;28:395-410.

17. Il bisogno socio-assistenziale. Disponibile: https://www. epicentro.iss.it/...//Sicilia_Gestione_Malattie_Croniche_V1\%20mar13.doc data ultima consultazione online; $03 / 02 / 2020$.

18. www.gurs.regione.sicilia.it data ultima consultazione online 03/02/2020.

19. pti.regione.sicilia.it $>$ portal $>$ page $>$ portal data ultima consultazione online 03/02/2020.

20. www.doesicilia.it. Piano Sanitario Regionale "Piano della Salute" 2010-2012.

21. Linee guida Cure domiciliari 2014. www.aspag.it > index.php > item > download. data ultima consultazione online 03/02/2020

22. Capitolato speciale. Servizio di assistenza domiciliare Sanitaria Integrata nel territorio della Azienda Sanitaria Domiciliare Sanitaria Integrata nel territorio della Azienda Sanitaria Provinciale di Agrigento. www.aspag.it > index.php > moduli-dsm > item > download. data ultima consultazione on line 04/02/2020

23. Gazzetta Ufficiale della Regione siciliana del 30 novembre 2007 , n. 56. data ultima consultazione online $03 / 02 / 2020$.

24. Linee guida regionali per l'accesso e il governo del sistema integrato delle cure domiciliari” DPRS 26 gennaio 2011. programmazione7-13.euroinfosicilia.it > Linee_guida_ADI_definitive. data ultima consultazione online $03 / 02 / 2020$

25. Linee Guida Regionali per l'accesso e il governo del sistema integrato delle cure domiciliari. Giugno 2010. data ultima consultazione online 03/02/2020.

26. Nuova caratterizzazione dell'assistenza territoriale domiciliare e degli interventi ospedalieri a domicilio. Ministero della Salute, Commissione Nazionale per la definizione e l'aggiornamento dei LEA.2006. www.salute.gov.it > portale > documentazione. data ultima consultazione online 04/02/2020.

27. Protocollo operativo per la presa in carico e gestione dei pazienti in Cure Palliative Domiciliari. www.aspag.it. data ultima consultazione online 04/02/2020.

28. Procedure Dimissioni Facilitate SPDC - ASP Agrigento. www.aspag.it > index.php > moduli-dsm > item > download. data ultima consultazione online 04/02/2020.

29. Accordo tra lo Stato, le Regioni e le Province Autonome di Trento e Bolzano del 15settembre 2016 in materia di "Piano Nazionale Cronicità"www.salute.gov.it/imgs/C 17 pubblicazioni 2584 allegato pdf 
30. Regione Piemonte, Piano nazionale cronicità. Recepimento Accordo Stato-Regioni 15.9.2016 e approvazione delle Linee di indirizzo regionali per le annualità 2018-2019. Documento in corso di discussione presso il Consiglio Regionale Piemonte. www.regione.piemonte.it/ sanita/cms2/documentazione/.../237-pianodella-cronicita

31. Deliberazione Direttore Generale ASL VC- Piemonten.200 del 20/03/2019 avente ad oggetto: "Accordo Aziendale per la medicina Generale Convenzionata" Anno 2018 - Integrazione Regione Piemonte - Deliberazione della Giunta Regionale 628.7.2008 n. 55 9323 avente ad oggetto "definizione delle modalità e dei criteri dell'utilizzo da parte delle Aziende Sanitarie Locali (A.S.L) e dei Soggetti gestori delle funzioni socio-assistenziali (EE.GG.), delle risorse assegnate a livello nazionale al "Fondo per le non autosufficienze
“ per l'anno 2007 ed attribuzione alla Regione Piemonte", B.U.R.P. n. 15 del 16.04.2009.

32. Legge Regionale FVG 26/2015: http://lexviewint.regione.fvg.it/FontiNormative/xml/XmlLex.aspx?anno= $2015 \&$ legge $=26 \& \mathrm{id}=\& \mathrm{fx}=$ lex\&ci $=0 \&$ lang $=$ multi\&idx $=$ ctrll

33. Legge Regionale del 31 marzo 2006, n. 6 art. 4

34. DGR 2151 dd. 29 ottobre 2015 "LINEE GUIDA PER LA GESTIONE DELLE RESIDENZE SANITARIE ASSISTENZIALI".

35. http://www.aas3.sanita.fvg.it/it/chi_siamo/atto.html

36. Brown I, Renwick R, Raphael D. Frailty: constructing a common meaning, definition, and conceptual framework. Int J Rehab Res 1995;18:93-102.

37. Fried LP. Frailty in older adults evidence for a phenotype. J Gerontol Ser A Biol Sci Med Sci 2001;56: M146-57. 


\title{
Analisi della survey Un giorno in Medicina. Un viaggio attraverso le opinioni degli infermieri ANİMO
}

\author{
Gabriella Bordin, ${ }^{1}$ Ruggero Pastorelli ${ }^{2}$ \\ ${ }^{1}$ Medicina Interna, Castelfranco Veneto (TV); ${ }^{2}$ Medicina Interna, Colleferro (RM), Italia
}

\begin{abstract}
Gli Infermieri italiani aderenti all'Associazione Italiana Infermieri di Medicina (ANİMO) sono stati coinvolti in una survey web-based finalizzata a raccogliere le opinioni e gli orientamenti di chi vive quotidianamente la realtà clinica, in merito a: i) gli indicatori ritenuti più idonei a valutare la qualità dell'assistenza, ii) gli aspetti dell'attività di reparto più soddisfacenti, importanti, peculiari e da modificare, iii) la propensione alla cura dei pazienti critici, iv) $\mathrm{i}$ rapporti tra le Unità Operative (UO) di Medicina Interna (MI) con l'Ospedale e il Dipartimento di Emergenza e Accettazione (DEA).

Sono stati raccolti 408 questionari con una rappresentatività regionale corrispondente alla numerosità dei soci iscritti.

L'elaborazione dei dati è stata condotta dal Centro Studi della Federazione Nazionale dei Dirigenti Ospedalieri Internisti (FADOI) ed i risultati confermano l'importanza data dagli Infermieri agli elementi caratterizzanti l'assistenza in medicina quali la visione globale della persona e la complessità, all'interno di un contesto in cui la tecnologia è strumentale. Restano fondamentali le competenze cliniche, la cultura del fine vita, la continuità assistenziale, la comunicazione ed il lavoro interdisciplinare.

I dati suggeriscono l'opportunità di rivedere i processi che hanno evidenziato maggiori criticità quali la modalità di esecuzione della visita collegiale ed i rapporti con l'Ospedale e con il DEA. Queste aree saranno tenute in considerazione da ANİMO nella
\end{abstract}

Corrispondente: Gabriella Bordin, Medicina Interna, via dei Carpani, 31033 Castelfranco Veneto (TV), Italia.

E-mail: g.bordin@libero.it

Articolo pubblicato secondo la Creative Commons Attribution NonCommercial 4.0 License (CC BY-NC 4.0).

${ }^{\circ}$ Copyright: the Author(s), 2020

Licensee PAGEPress, Italy

QUADERNI - Italian Journal of Medicine 2020; 8(3):55-63 programmazione delle attività societarie e nel confronto con le istituzioni per definire iniziative atte a migliorare il contesto delle MI in cui gli Infermieri erogano assistenza.

\section{Introduzione}

L'aumento della sopravvivenza e la presenza di fasce di popolazione fragili ha determinato in questi anni un'accresciuta richiesta di salute che si è riversata su tutti i settori della Sanità ma che ha trovato la sua valvola di sfogo privilegiata negli Ospedali ed in particolare nelle $\mathrm{MI}^{1}$ cui afferiscono pazienti con bisogni assistenziali diversi e con flussi non programmabili. Sono in genere adulti, anziani, pluripatologici, con malattie croniche degenerative, clinicamente instabili, con limitata speranza di vita, spesso fragili e a rischio di perdita dell'autosufficienza con conseguenti risvolti sociali che rendono difficile la dimissione e a rischio di ricoveri ripetuti. ${ }^{2}$

Questo scenario epidemiologico si inquadra in un trend di progressiva riduzione della dotazione di posti letto in ospedale, con richiesta di limitare la durata della degenza senza che l'auspicato sviluppo delle cure territoriali abbia portato, ad oggi, risultati percepibili sulla appropriatezza dei ricoveri ospedalieri. Il sistema di accoglienza dei ricoveri urgenti va spesso in crisi, con conseguente sovraffollamento sia dei DEA che dei reparti di degenza, particolarmente in area medica. Il sovraffollamento ha conseguenze importanti sia sul clima organizzativo che sulle persone, influenza l'esito delle cure e può modificare la soddisfazione dei pazienti e del personale di assistenza. Tra le motivazioni sottese all'aumentato turnover del personale infermieristico nei reparti di medicina sono comprese ragioni quali un eccessivo carico di lavoro, un lavoro di trincea, il troppo tempo speso a fare il posto letto per nuovi pazienti nuovi. L'aumentato turnover del personale infermieristico non facilita il consolidamento definitivo del gruppo professionale in un lavoro di squadra stabile, induce la perdita della continuità assistenziale e rende incerta la qualità della cura. ${ }^{3}$

Chi lavora in MI deve sviluppare una gamma di 
competenze eterogenee che vanno dall'assistenza al paziente critico (ventilazione non invasiva, complicanze settiche, gestione di cateteri venosi centrali, procedure invasive, ecografia bedside, ecc.) al reinserimento sul territorio del paziente fragile, dai percorsi assistenziali complessi alla gestione della terminalità, dalla gestione dei posti letto alle competenze relazionali.

Nella gestione della complessità descritta giocano un ruolo importante la competenza clinica degli Infermieri, l'attenzione alla sicurezza e alla qualità delle cure, il rapporto all'interno del gruppo di lavoro, i rapporti con le altre strutture in particolar modo il DEA e la direzione.

Tali premesse hanno costituito una valida motivazione ad aderire alla proposta di FADOI di condurre una survey, sulla scorta di quanto già fatto con gli Internisti, volta a rilevare una serie di opinioni e di orientamenti degli Infermieri delle MI, su argomenti quali: i) gli indicatori più idonei a valutare la qualità della cura in MI; ii) gli aspetti più soddisfacenti e più importanti dell'attività di reparto, nonché gli aspetti peculiari e quelli da modificare; iii) la propensione alla cura dei pazienti critici; iv) i rapporti tra le UO di MI, l'Ospedale e il DEA.

I dati potrebbero fornire una rappresentazione nazionale degli orientamenti degli Infermieri ANİMO sui temi oggetto della survey e potrebbero orientare le iniziative societarie nelle attività formative e nel confronto con le istituzioni a completamento di quanto già emerge dalla letteratura.

\section{Materiali e Metodi}

Un gruppo di lavoro ANÌMO, utilizzando il metodo Delphi, ha valutato gli item presenti nella survey FADOI e, per garantire che i dati delle indagini fossero comparabili, ne è stata mantenuta la struttura generale, alcuni item sono stati modificati e nove aggiunti per renderli pertinenti all'attività infermieristica.

La survey è stata condotta on line dal 3.10.19 all'8.11.19 e prevedeva la compilazione da parte dei soci $\mathrm{ANIMMO}$, di un questionario costruito su quattro aree tematiche volte ad individuare: i) gli indicatori più idonei a valutare la qualità dell'assistenza (38 item) questionario $\mathrm{A}$; ii) gli aspetti dell'attività di reparto più soddisfacenti (10 item), più importanti (13 item), peculiari (6 item), da modificare (12 item) e la cura dei pazienti critici (4 item) - questionario B; iii) le opinioni degli Infermieri sui rapporti tra le UO di MI, l'Ospedale (5 item) e il DEA (4 item) - questionario C.

Ad ogni item è stato chiesto di assegnare un valore tra 0 e 10 ( 0 irrilevante, 10 estremamente importante).

Inoltre sono stati raccolti dati quali l'età, il ruolo e alcune caratteristiche dell'ospedale (localizzazione regionale, numero posti letto, caratteristiche del DEA).

I dati sono stati trattati in forma anonima ed elaborati dal Centro Studi FADOI.

\section{Risultati}

Le aree regionali di appartenenza dei soci, i dati generali e le caratteristiche di contesto sono riassunti nella Tabella 1 e 2.

Sono stati raccolti 408 questionari di cui 63 compilati parzialmente con una rappresentatività regionale corrispondente alla numerosità dei soci iscritti al 31.12.18: nord 207 (51\%), centro-nord 75 (18\%), centro-sud $50(12 \%)$, isole $76(19 \%)$. Le fasce di età maggiormente rappresentative sono quelle che comprendono i 30-45 anni (40\%) e 46-55 anni (41\%), il $25 \%$ dei rispondenti sono infermieri coordinatori.

I rispondenti lavorano in ospedali con un numero di posti letto inferiore a $200(37 \%)$, tra 200 e 400 $(37 \%)$ e superiore a $400(36 \%)$. Il 36\% degli ospedali sono sede di DEA di I livello (36\%) II livello (21\%) e Pronto Soccorso (27\%).

\section{Questionario A. Indicatori più idonei a valutare la qualità dell'assistenza}

La Tabella 3 presenta la sintesi dei punteggi delle 38 domande relative agli indicatori ritenuti importanti per la qualità delle cure, aggregati per aree tematiche (attività, risorse e organizzazione, processi, esiti). I valori sono distribuiti in modo omogeneo all'interno della fascia che va da 7 a 9, mentre la Tabella 4 riporta i 13 indicatori che hanno ottenuto il punteggio più elevato $(\geq 8)$.

Il punteggio medio assegnato agli indicatori correlati alle risorse $(8,1)$ processi $(8,2)$, ed esiti $(8,1)$ è risultato maggiore di quello riportato dagli indicatori

Tabella 1. Risposte per macroarea regionale.

\begin{tabular}{|c|c|c|c|c|c|}
\hline & Totale & $\%$ & Complete & Parziali & Iscritti \\
\hline Nord & 207 & $51 \%$ & 174 & 33 & 337 \\
\hline Centro-Nord & 75 & $18 \%$ & 66 & 9 & 87 \\
\hline Centro & 50 & $12 \%$ & 41 & 9 & 75 \\
\hline Sud e Isole & 76 & $19 \%$ & 64 & 12 & 124 \\
\hline
\end{tabular}

Nord: Piemonte, Liguria, Lombardia, Veneto, Trentino Alto Adige, Friuli Venezia Giulia; Centro Nord: Toscana, Emilia Romagna; Centro: Lazio, Marche, Umbria, Abruzzo, Molise ; Sud e Isole: Campania, Basilicata, Puglia, Calabria, Sicilia, Sardegna. 
amministrativi correlati all'attività $(7,4)$. Alcuni partecipanti hanno indicato come ulteriori indicatori di qualità delle cure: l'utilizzo di scale di rilevazione dell'indice di allerta precoce (MEWS) e di valutazione multidimensionale, il clima relazionale dell'equipe, la presenza di sistemi di monitoraggio della soddisfazione lavorativa negli operatori, il turnover del personale.

\section{Questionario B. Aspetti più soddisfacenti, importanti, peculiari e da modificare dell'attività di reparto}

La Tabella 5 riporta i punteggi relativi agli aspetti ritenuti rispettivamente più soddisfacenti, importanti, peculiari e da modificare. La distribuzione dei punteggi va da 5,6 a 8,7. Il punteggio medio relativo agli aspetti più soddisfacenti $(6,7)$ è risultato inferiore a quello relativo agli aspetti peculiari $(8,1)$, da modificare $(8,2)$ e più importanti $(8)$.

Le Figure 1-2-3 mostrano come i punteggi siano distribuiti in modo uniforme per i diversi item. Solo la visita collegiale e la possibilità di svolgere tecniche strumentali a scopo assistenziale come accessi vascolari, cateterismo vescicale (CV), sondino nasogastrico (SNG) e la ventilazione non invasiva (NIV) mostrano punteggi più bassi rispetto agli item che indagano tematiche legate al rapporto con il paziente, alla collaborazione con gli altri componenti dell'equipe e ai processi tipici dell'attività infermieristica quali la pianificazione assistenziale, la dimissione, le consegne.

La Figura 4 fa riferimento agli aspetti da modificare con punteggi omogenei per gli item indagati a parte l'organizzazione per compiti che riporta il punteggio più basso $(6,9)$.

\section{La cura del paziente critici}

Il $43 \%$ dei partecipanti lavora in UO dotate di posti letto monitorizzati ed il $92 \%$ ritiene che tale supporto tecnologico possa essere un vantaggio per l'attività assistenziale. Il 93\% si dichiara favorevole ad essere in prima linea insieme all'internista nella gestione di pazienti critici e instabili e disposto a formarsi sulla gestione del paziente critico in MI.

Tabella 2. Dati generali e di contesto.

\begin{tabular}{lcc}
\hline & Totale & $\%$ \\
\hline$<30$ & Età partecipanti & \\
\hline $30-45$ & 32 & $8 \%$ \\
\hline $46-55$ & 164 & $40 \%$ \\
\hline$\geq 56$ & 166 & $41 \%$ \\
\hline \multicolumn{4}{c}{46} & $11 \%$ \\
\hline $200-400$ & Ospedale: posti letto \\
\hline$>400$ & 112 & $27 \%$ \\
\hline \multicolumn{4}{c}{149} \\
\hline DEA I livello & 147 & $37 \%$ \\
\hline DEA II livello & 148 & $36 \%$ \\
\hline PS & 87 & $36 \%$ \\
\hline Altro & 110 & $21 \%$ \\
\hline
\end{tabular}

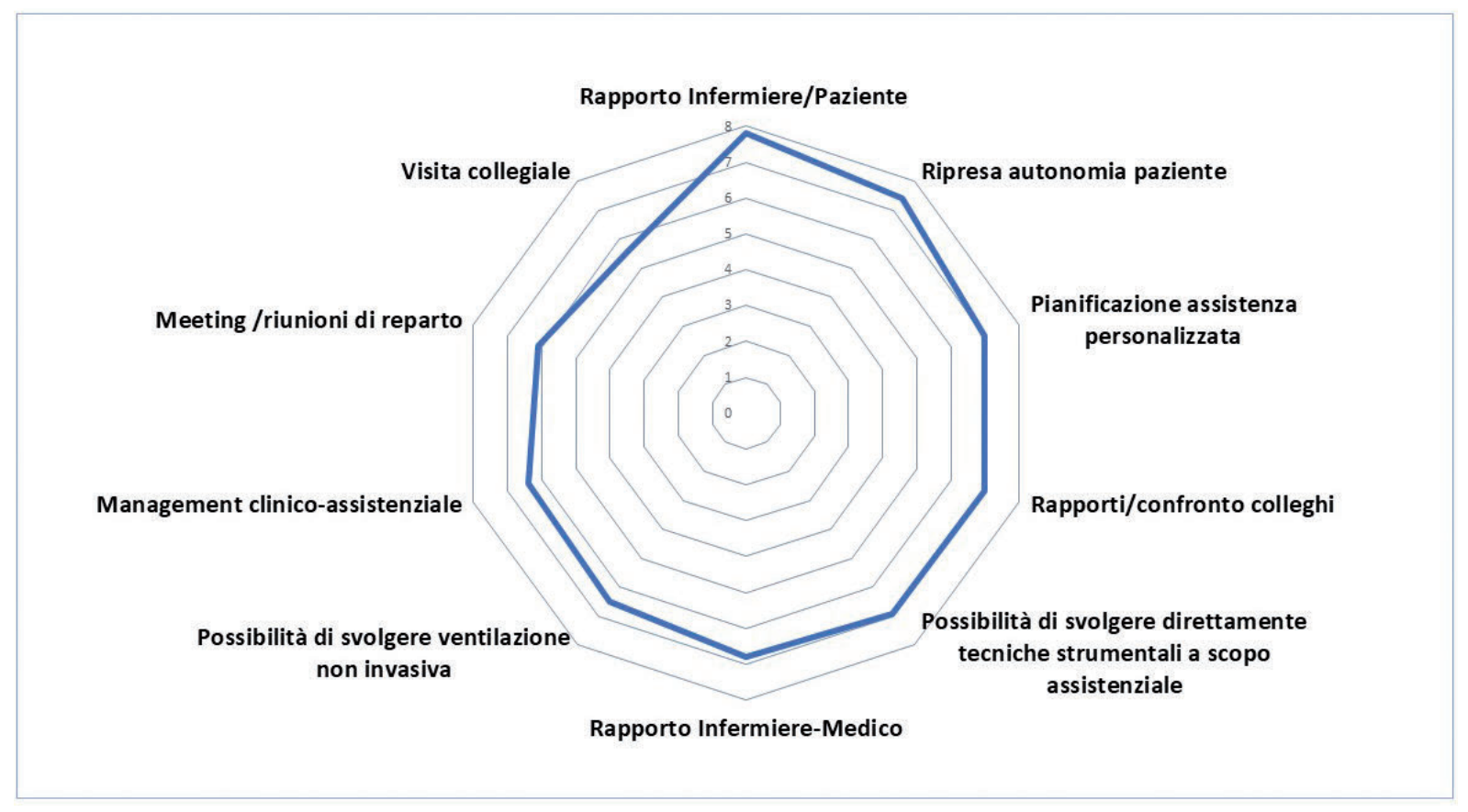

Figura 1. Risultati Questionario B. Aspetto più soddisfacente. 


\section{I rapporti tra le UO di Medicina Interna, l'Ospedale e il DEA}

Il 73\% degli Infermieri percepisce come importante, non ancillare il proprio ruolo all'interno delle $\mathrm{UO}$, ritenute organizzazioni che forniscono una visione forte della medicina interna (54\%), mentre ritengono nel $72 \%$ dei casi che gli atti e le decisioni della Direzione Strategica non tengano in giusta considerazione l'attività da loro svolta.

Nell'ultimo anno il 32\% dei partecipanti dichiara di essere stato chiamato dalle Direzioni Mediche nell'elaborazione di percorsi/protocolli mentre il $49 \%$ e il $19 \%$ rispettivamente è coinvolto raramente o mai.

Il 79\% dei partecipanti afferma che nelle proprie UO sono assegnati Infermieri senza una formazione

Tabella 3. Questionario A. Indicatori di qualità delle cure.

\begin{tabular}{|c|c|c|}
\hline \multicolumn{3}{|c|}{ Indicatori amministrativi correlati all'attività } \\
\hline Numero di re-ricoveri entro un mese & & 8,0 \\
\hline Percentuale di dimissioni a domicilio & & 7,5 \\
\hline Numero di dimissioni l'anno & & 7,4 \\
\hline Degenza media & & 7,3 \\
\hline Percentuale di pazienti ricoverati dal Pronto Soccorso & & 7,3 \\
\hline Numero di pazienti appoggiati in altro reparto & & 7,1 \\
\hline Numero di pazienti in letti aggiunti in reparto & & 7,1 \\
\hline & Punteggio medio & 7,4 \\
\hline \multicolumn{3}{|c|}{ Indicatori correlati alle risorse e organizzazione } \\
\hline Rapporto n. infermieri/n. pazienti & & 9,0 \\
\hline Presenza di personale di supporto & & 8,6 \\
\hline Individuazione dell'infermiere che si prende in carico il paziente & & 8,3 \\
\hline Individuazione del Medico che prende in carico il paziente & & 8,2 \\
\hline Presenza in visita di uno stesso medico per almeno 5 giorni/settimana & & 8,2 \\
\hline Rapporto n. medici/n. pazienti & & 7,9 \\
\hline Presenza dello stesso Infermiere nella visita quotidiana & & 7,3 \\
\hline Presenza del Coordinatore nelle attività cliniche & & 7,0 \\
\hline & Punteggio medio & 8,1 \\
\hline \multicolumn{3}{|c|}{ Indicatori correlati ai processi } \\
\hline Gestione del fine vita & & 8,9 \\
\hline Modello organizzativo-assistenziale centrato sulla persona & & 8,7 \\
\hline Organizzazione per continuità assistenziale & & 8,6 \\
\hline Misurazione dolore con scale validate & & 8,6 \\
\hline Gestione rischio clinico & & 8,5 \\
\hline Riunioni d'equipe & & 8,3 \\
\hline Percorsi diagnostico terapeutici assistenziali & & 8,3 \\
\hline \multicolumn{3}{|c|}{ Possibilità di svolgere tecniche strumentali a scopo assistenziale (ecografia per accessi venosi, catetere vescicale, sondino naso-gastrico) 8,3} \\
\hline Lettera di dimissione medico-infermieristica strutturata & & 8,2 \\
\hline Presenza di sistemi di valutazione della complessità assistenziale & & 8 \\
\hline Possibilità di effettuare ventilazione non invasiva & & 7,8 \\
\hline Presenza di sistemi di valutazione degli outcome assistenziali & & 7,8 \\
\hline Attrattività dell'unità operativa come luogo di lavoro & & 7,6 \\
\hline Presenza di sistemi di monitoraggio del deterioramento clinico & & 7,6 \\
\hline & Punteggio medio & 8,2 \\
\hline \multicolumn{3}{|c|}{ Indicatori correlati agli esiti } \\
\hline Grado di soddisfazione dei pazienti & & 8,8 \\
\hline Ripresa dell'autonomia funzionale e di gestione del proprio stato di salute & & 8,6 \\
\hline Incidenza infezioni correlate all'assistenza & & 8,6 \\
\hline Incidenza delle lesioni da pressione & & 8,5 \\
\hline Numero delle cadute dei pazienti & & 8,0 \\
\hline Percentuale di pazienti sottoposti a cateterismo vescicale & & 7,3 \\
\hline Ricorso alla contenzione eccezionale/temporaneo solo a tutela sicurezza & & 7,3 \\
\hline Numero dei contenziosi & & 7,2 \\
\hline Percentuale di mortalità & & 7,2 \\
\hline & Punteggio medio & 8,1 \\
\hline
\end{tabular}


specifica mentre questo accade raramente per il 17\% e mai per il 4\% dei casi (Figura 5).

Un percorso di condivisione con il PS per la definizione del flusso dei ricoveri o la consulenza strutturata nelle emergenze/urgenze è ritenuto molto importante dal 98\% dei partecipanti che, tenendo conto di aree di degenza nel DEA, considerano i ricoveri appropriati nel $46 \%$ dei casi mentre per il $45 \%$ l'appropriatezza è minima e per il $9 \%$ nulla.
Gli infermieri dichiarano di essere contattati dai colleghi del DEA per il passaggio di informazioni assistenziali prima dell'arrivo della persona in reparto sempre $(22 \%)$, raramente $(47 \%)$, mai (31\%), e che il verbale del Pronto Soccorso (PS) riporta informazioni utili alla presa in carico infermieristica e alla continuità assistenziale sempre (24\%), raramente (61\%), mai (15\%) (Figura 6).

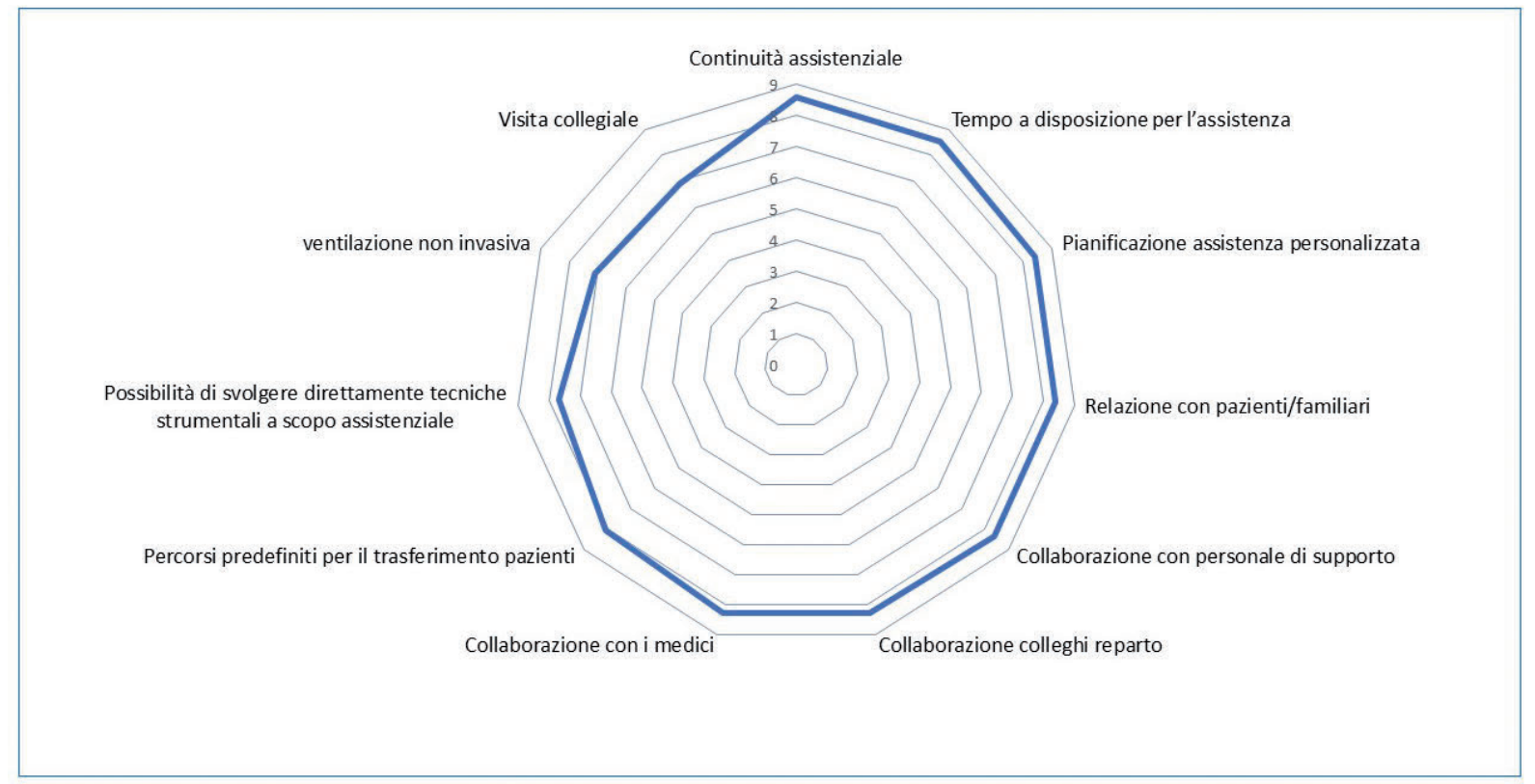

Figura 2. Risultati Questionario B. Aspetto più importante.

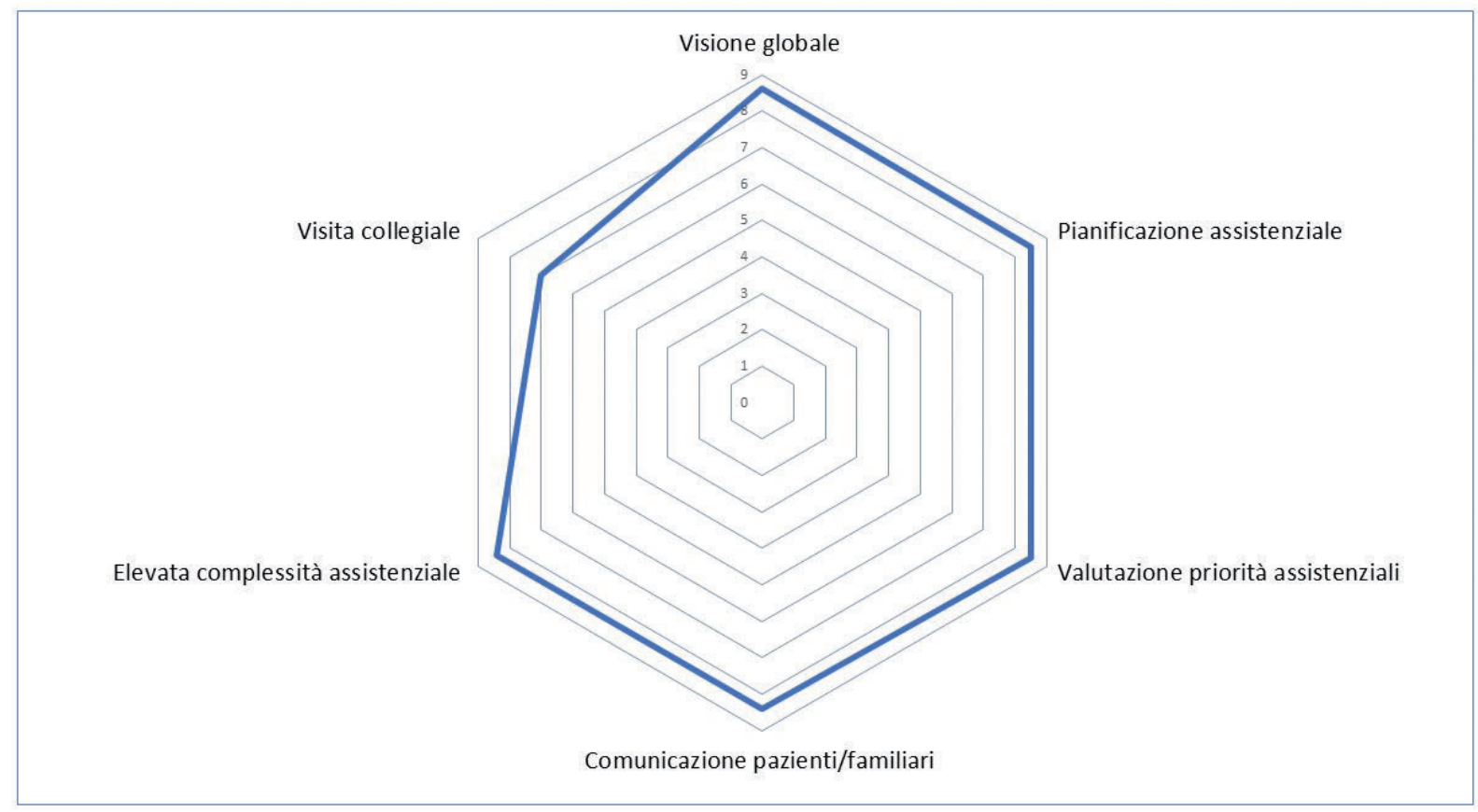

Figura 3. Risultati Questionario B. Aspetto peculiare. 


\section{Discussione}

La survey ha visto la partecipazione del $65 \%$ degli iscritti e per questo può essere considerata rappresentativa dell'orientamento dei soci su tutto il territorio nazionale.

La distribuzione omogenea dei punteggi dati agli item proposti come indicatori di qualità delle cure e a quelli che fanno riferimento agli aspetti importanti e peculiari dell'attività di reparto esprime un consenso, che poteva non essere scontato, nei confronti degli elementi caratterizzanti l'attività degli Infermieri che lavorano nelle MI.

Per quanto riguarda gli indicatori della qualità della cura l'orientamento degli Infermieri tende a con- siderare maggiormente quelli orientati agli esiti, ai processi e alle risorse piuttosto che verso quelli di carattere amministrativo di prassi proposti in ambito aziendale. Questo sottolinea un diverso significato dato dai professionisti alla produzione di salute e qualità della cura rispetto ai decisori istituzionali orientati a prospettive di efficienza.

Il clima relazionale dell'equipe e la presenza di sistemi di monitoraggio della soddisfazione lavorativa sono stati citati da alcuni partecipanti come ulteriori indicatori di qualità non compresi negli item proposti. Nelle MI questi fattori devono essere considerati con attenzione per il peso che hanno sull'intenzione di lasciare il posto di lavoro o la professione, come evidenziato da Sasso, nello studio

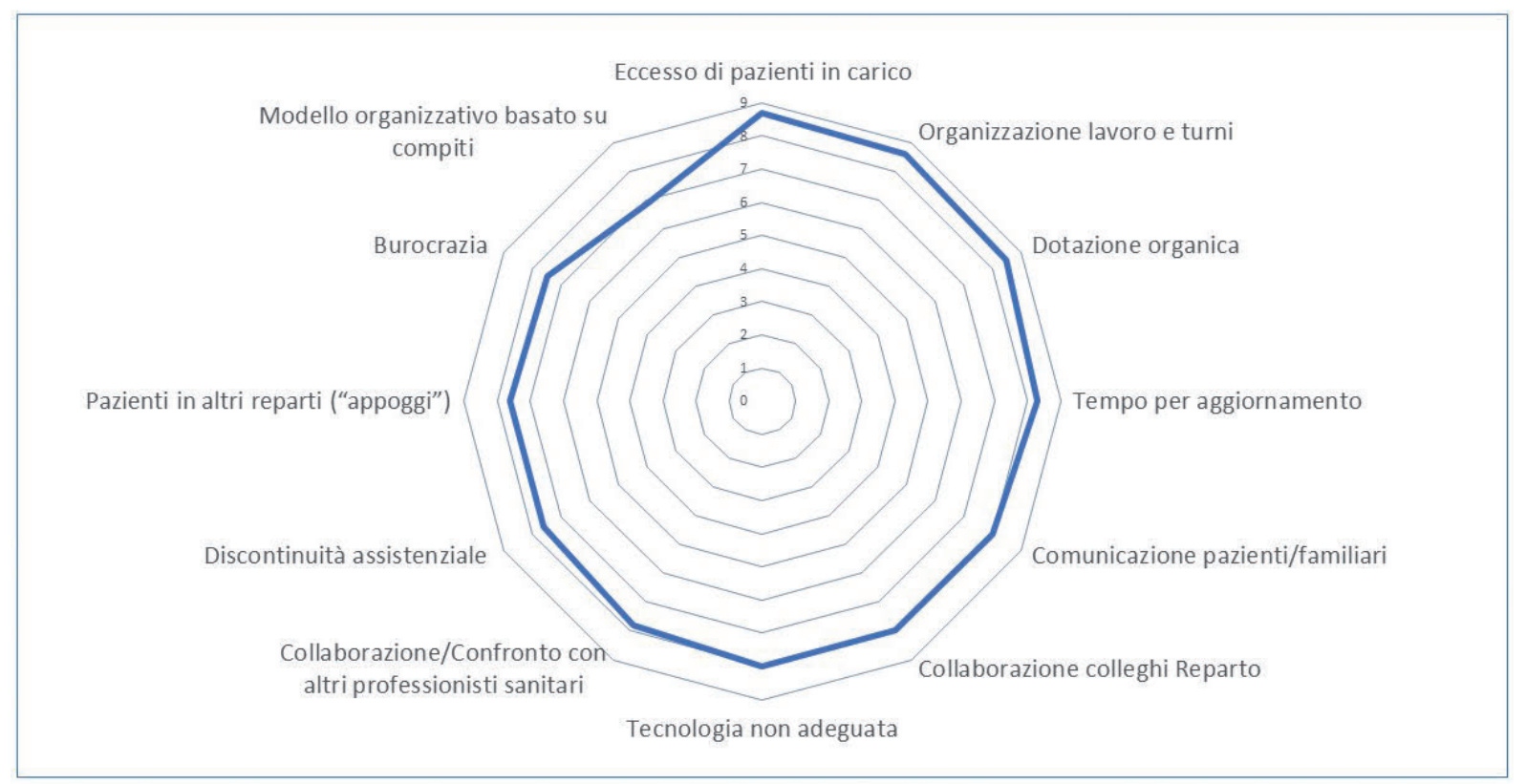

Figura 4. Risultati Questionario B. Aspetto da modificare.

Tabella 4. Indicatori con il punteggio più elevato $(>8)$.

\begin{tabular}{ll}
\hline Rapporto n. infermieri/n. pazienti & 9,0 \\
\hline Gestione del fine vita & 8,9 \\
\hline Grado di soddisfazione dei pazienti & 8,8 \\
\hline Organizzazione per continuità assistenziale & 8,6 \\
\hline Gestione rischio clinico & 8,5 \\
\hline Frequenza insorgenza delle lesioni da pressione & 8,5 \\
\hline Riunioni d'equipe & 8,3 \\
\hline Percorsi diagnostico terapeutici assistenziali) & 8,3 \\
\hline Possibilità di svolgere direttamente tecniche strumentali a scopo assistenziale (ecografia per accessi vascolari, cateterismo vescicale, & 8,3 \\
\hline sondino NG, ...) & 8,2 \\
\hline Lettera di dimissione medico-infermieristica strutturata \\
\begin{tabular}{l} 
Individuazione del Medico che prende in carico il paziente \\
\hline Presenza in visita di uno stesso medico per almeno 5 giorni /settimana
\end{tabular} \\
\hline Numero delle cadute dei pazienti
\end{tabular}


che esplora l'intention to leave (ITL) da parte degli Infermieri. ${ }^{4}$

Relativamente agli aspetti dell'attività più soddisfacenti, più importanti, peculiari e da modificare gli elementi che hanno ottenuto un punteggio più basso sono la possibilità di svolgere tecniche strumentali a scopo assistenziale (accessi vascolari, CV, SNG, NIV), e la visita collegiale.

Tabella 5. Questionario B.

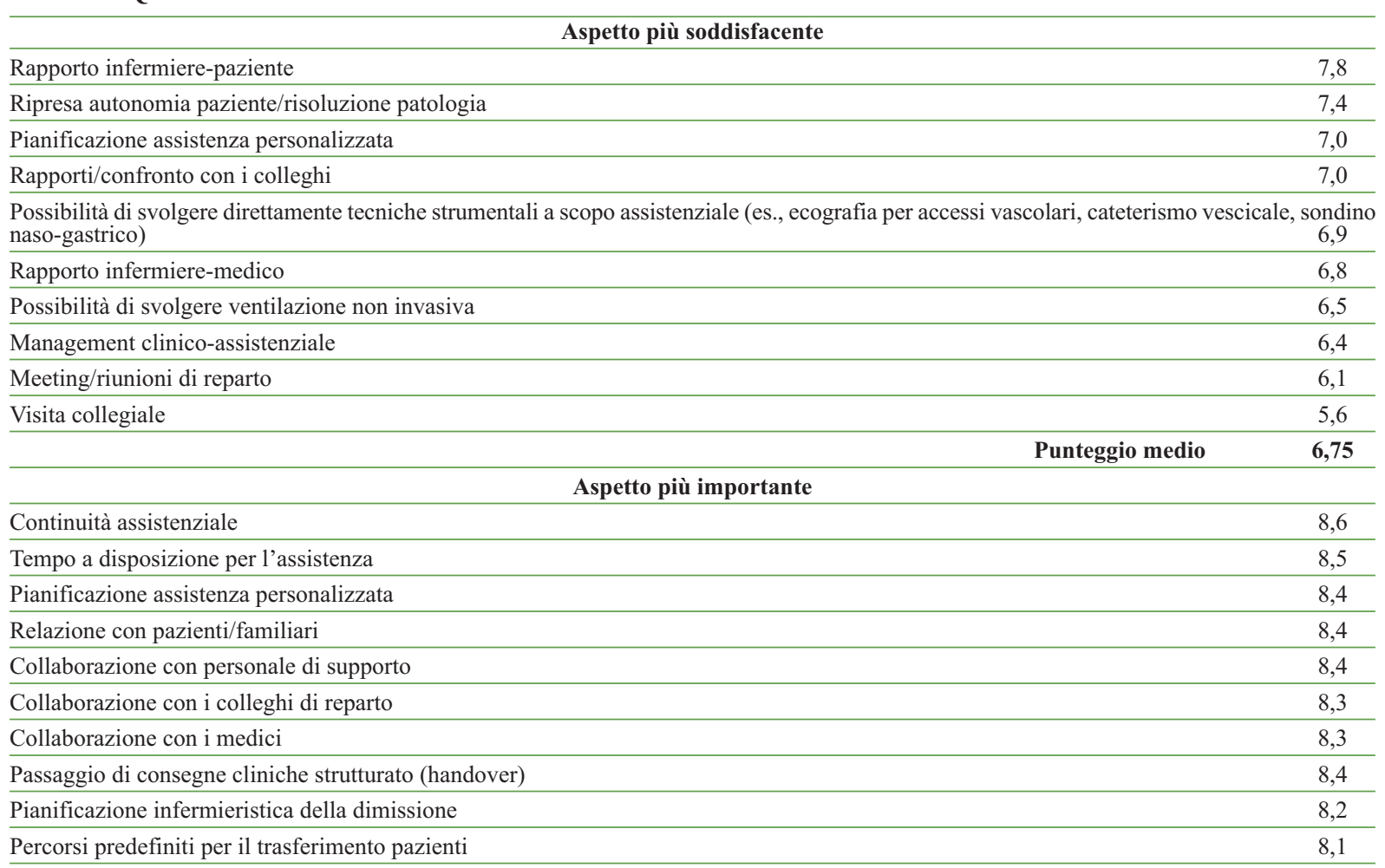

Possibilità di svolgere direttamente tecniche strumentali a scopo assistenziale (accessi vascolari, cateterismo vescicale, sondino naso gastrico)

Ventilazione non invasiva

Visita collegiale

6,9
8,1

\begin{tabular}{lrr} 
& Punteggio medio & \\
\hline & Aspetto peculiare & \\
\hline Visione globale & & \\
\hline Pianificazione assistenziale & 8,6 \\
\hline Valutazione priorità assistenziali & 8,5 \\
\hline Comunicazione pazienti/familiari & 8,5 \\
\hline Elevata complessità assistenziale & 8,4 \\
\hline Visita collegiale & & 8,4 \\
\hline & & 7,0 \\
\hline
\end{tabular}

Eccesso di pazienti in carico

Aspetto da modificare

Organizzazione lavoro e turni

Organizzazione lavoro e turni

Dotazione organica

Tempo per aggiornamento

Comunicazione pazienti/familiari

Collaborazione colleghi reparto

Tecnologia non adeguata

Collaborazione/confronto con altri professionisti sanitari

Discontinuità assistenziale

Pazienti in altri reparti (appoggi)

Burocrazia

Modello organizzativo basato su compiti 
Non sorprende che gli Infermieri considerino le tecniche meno importanti e peculiari della ripresa dell'autonomia della persona, della relazione con l'assistito, della collaborazione interna all'equipe, della pianificazione assistenziale mentre merita una riflessione particolare il peso dato alla visita collegiale. Il Royal College of Nursing definisce il giro visita una attività clinica complessa, cruciale per un'assistenza sanitaria di alta qualità, sicura e puntuale ${ }^{5} \mathrm{e}$ i soci di ANİMO non possono non condividere questa affermazione.
Gli Infermieri sono responsabili dell'assistenza e il loro coinvolgimento nella revisione clinica quotidiana al letto del paziente è fondamentale. Tuttavia esiste molta variabilità nella condotta del giro visita $\mathrm{e}$ nel ruolo attribuito alla presenza dell'Infermiere. Se i tempi della visita medica sono troppo lunghi ed il contributo dell'Infermiere non è valorizzato, la visita collegiale perde di significato e toglie tempo assistenziale all'Infermiere. È necessario un approccio organizzativo che garantisca adeguate risorse e flussi di lavoro,

Gli atti e le decisioni della Direzione Strategica tengono in giusto conto l'attività che svolgi?

L'organizzazione della tua UO fornisce una visione "forte" Medicina Interna ?

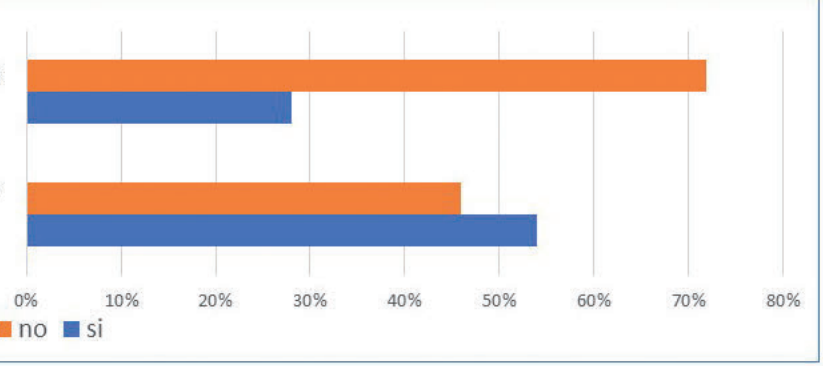

Si verifica l'assegnazione alla vostra unità operativa di Infermieri non formati in Medicina Interna?

Nell'ultimo anno i percorsi/protocolli validati dalla direzione sanitaria hanno previsto la presenza dell'Infermiere di Medicina Interna?

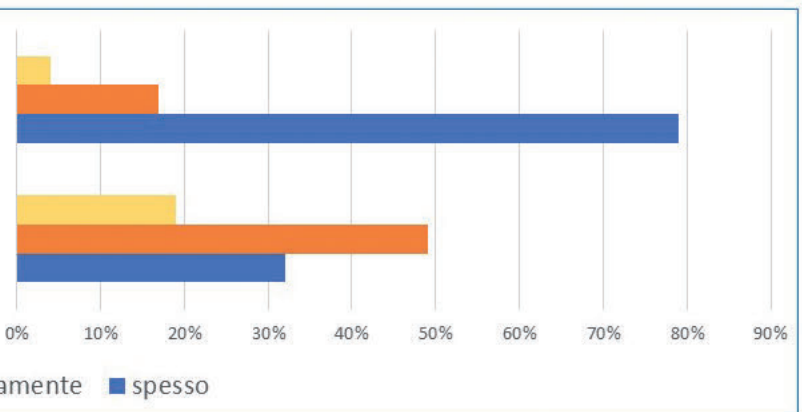

Figura 5. Questionario C. Rapporti con l'Ospedale.

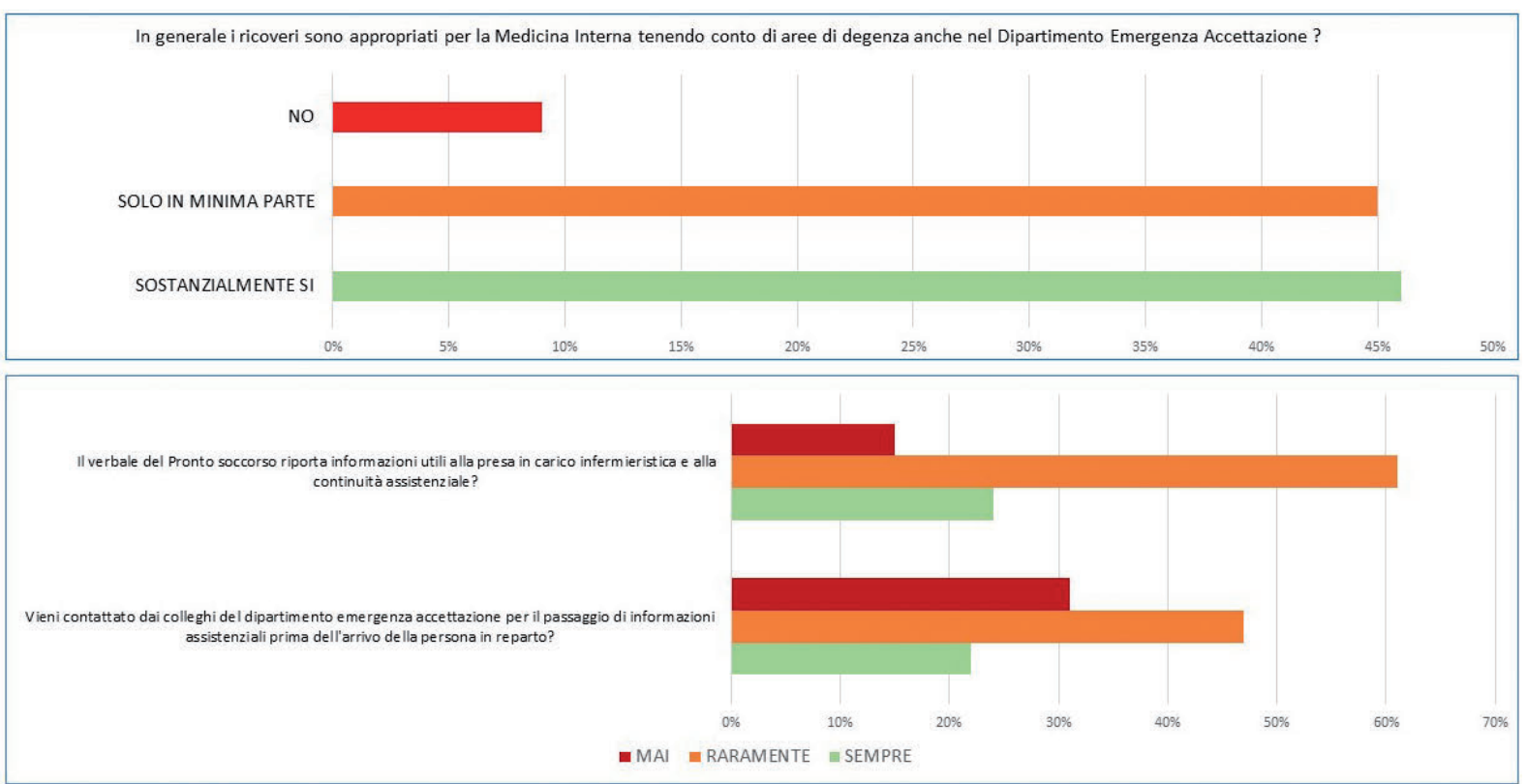

Figura 6. Questionario C. Rapporti con il Dipartimento Emergenza e Accettazione. 
comunicazione centrata sul paziente, liste di controllo per la sicurezza della qualità ${ }^{6}$ affinchè la visita collegiale possa trasformarsi in un momento formativo per gli appartenenti al gruppo multidisciplinare, migliorando l'assistenza erogata al paziente e la comunicazione fra professionisti. ${ }^{7}$

La propensione ad assistere pazienti critici e la disponibilità ad essere formati in quast'area sottende la consapevolezza della necessità di acquisire competenza nell'assistere una casistica di pazienti sempre più frequesti in medicina. Circa il $10 \%$ dei pazienti ricoverati in $\mathrm{MI}$ ha necessità di un setting assistenziale che garantisca trattamenti di alta intensità e necessità di monitoraggio specifico. ${ }^{8}$ Nonostante ciò le UO dotate di posti letto monitorizzati sono in minoranza e questo sottolinea la necessità che si renda disponibile una tecnologia adeguata a supporto dell'attività clinica, oltre all'utilizzo di scale di rilevazione dell'indice di allerta precoce ai fini di garantire cure appropriate e sicure.

In quest'ottica il rapporto con il DEA diventa fondamentale in termini di percorsi condivisi, modalità comunicative adeguate, attenzione all'appropriatezza dei ricoveri.

La maggior parte dei partecipanti (73\%) percepisce come importante, non ancillare il proprio ruolo all'interno delle unità operative, ritenute organizzazioni che forniscono una visione forte della medicina (54\%).

La forte identità interna non trova riscontro nella considerazione da parte della Direzione che si traduce nel limitato coinvolgimento nella stesura di documenti di indirizzo o nell'assegnazione di uno staffing quantiqualitativamente inadeguato, mentre la letteratura annovera la partecipazione alle attività aziendali tra $\mathrm{i}$ fattori che diminuiscono il rischio di $\mathrm{ITL}^{4}$ ed è noto che il numero degli infermieri, ma anche il loro livello di formazione e di esperienza professionale, incidono sul tasso di mortalità e sugli esiti assistenziali in generale..$^{9,10}$

Questi elementi critici trovano conferma nei punteggi dati agli aspetti da modificare: carico di lavoro eccessivi, modelli organizzativi orientati all'efficientismo, burocrazia, tempi inadeguati per la formazione e la relazione con l'assistito e i colleghi.

\section{Conclusioni}

La survey, pur con i limiti legati al tipo di indagine, ha raccolto le opinioni degli Infermieri delle MI, confermando l'importanza degli elementi caratterizzanti l'assistenza in medicina quali la visione globale della persona e la complessità, all'interno di un contesto in cui la tecnologia è strumentale mentre restano fondamentali le competenze cliniche, la continuità assistenziale, la cultura del fine vita, la comunicazione ed il lavoro interdisciplinare.
Il rapporto con il DEA, il riconoscimento e valorizzazione dell'attività svolta dagli Infermieri nelle medicine da parte delle direzioni aziendali rappresentano due ambiti di miglioramento che non possono essere sottovalutati per le implicazioni che hanno sulla qualità dell'assistenza e sulla soddisfazione lavorativa.

Il minor grado di soddisfazione e importanza espresso nei confronti della visita collegiale merita una discussione interna e condivisa tra ANİMO e FADOI per rivedere le modalità di realizzazione di questa attività fondamentale e caratterizzante la MI.

I risultati della survey definiscono aree tematiche che ANİMO deve considerare nella programmazione di attività formative, per mantenere alta la competenza degli Infermieri, e nel confronto con le istituzioni per definire iniziative atte a migliorare il contesto delle medicine interne in cui gli Infermieri erogano assistenza.

\section{Ringraziamenti}

Annachiara Crespi, Clinical Project Manager, Centro Studi FADOI.

\section{Bibliografia}

1. Panigada G, Chiti I. Aspetti clinico-organizzativi nella degenza medica ospedaliera in Italia: il ruolo della Medicina Interna nel dipartimento medico e continuità assistenziale Quaderni Ital J Med 2015;3:499-502.

2. Bertoncini F, Gatta C, Pentella G. Nursing clinical competence in area medica. Quaderni Ital J Med 2018;6:1-51.

3. Nardi R, Gardellini A, Iori I. Sovraffollamento nei reparti di degenza e clinical risk management in Medicina Interna: soluzioni solamente strutturali o interventi sistemici sui processi assistenziali? Ital J Med 2009;3:3-8.

4. Sasso L, Bagnasco A, Catania G, et al. Push and pull factors of nurses'intention to leave. J Nurs Manag 2019;27:946-54.

5. Royal College of Physicians, Royal College of Nursing. Ward rounds in medicine: principles for best practice. London: RCP; 2012.

6. Stein J, Payne C, Methvin A, et al. Reorganizing a Hospital Ward as an Accountable Care Unit. J Hospital Med 2015;10:36-40.

7. Simonetti V, Comparcini D, Tesei L, et al. Il giro visita: una prassi che garantisce la qualità? Una revisione integrativa della letteratura. L'infermiere 2019;56:6:e106-19.

8. Bartolomei C, Cei M. L'allocazione dei pazienti in un reparto di Medicina Interna organizzato per intensità di cure: lo studio ADOIT Tri-Co (Triage di Corridoio) Ital J Med 2007;2:31-9.

9. Sasso L, Bagnasco A, Zanini M, et al. The general results of the RN4CAST survey in Italy. J Adv Nurs 2017;73:2028-30.

10. Pentella G, Tesei L. Rischio clinico: livelli di staffing infermieristico ed esiti correlati alle cure infermieristiche. Quaderni Ital J Med 2019;7:19-24. 


\title{
Un giorno in medicina: i risultati delle Survey FADOI e ANÌMO: quanto vicini, quanto lontani
}

\author{
Ada Maffettone, ${ }^{1}$ Gabriella Bordin ${ }^{2}$ \\ ${ }^{1}$ UOC Medicina Cardiovascolare e Dismetabolica, AO Ospedali dei Colli, Napoli; ${ }^{2}$ Medicina Interna, Castelfranco Veneto \\ (TV), Italia
}

\section{Introduzione}

I Medici aderenti alla Federazione delle Associazioni Dirigenti Internisti Ospedalieri (FADOI) e gli Infermieri aderenti all'Associazione Italiana Infermieri di Medicina (ANİMO) sono stati coinvolti in una survey web-based finalizzata a raccogliere le opinioni e gli orientamenti di chi vive quotidianamente la realtà clinica, in merito a: i) gli indicatori ritenuti più idonei a valutare la qualità dell'assistenza; ii) gli aspetti dell'attività di reparto più soddisfacenti, importanti, peculiari e da modificare; iii) la propensione alla cura dei pazienti critici, iv) i rapporti tra le Unità Operative (UO) di Medicina Interna (MI) con l'Ospedale e il Dipartimento di Emergenza e Accettazione (DEA).

Hanno partecipato 740 Medici e 408 Infermieri con una rappresentatività regionale corrispondente alla numerosità dei soci iscritti.

L'elaborazione dei dati è stata condotta dal Centro Studi FADOI.

I risultati confermano l'importanza data dai professionisti di entrambe le società agli elementi che caratterizzano la cura e l'assistenza in Medicina quali la visione globale della persona e la complessità, all'interno di un contesto in cui la tecnologia è strumentale all'attività. Restano fondamentali le competenze cliniche, la cultura del fine vita, la continuità assistenziale, la comunicazione ed il lavoro interdisciplinare.

I dati indicano gli elementi che hanno evidenziato maggiori criticità quali la modalità di esecuzione della

Corrispondente: Ada Maffettone, UOC Medicina Cardiovascolare e Dismetabolica, AO Ospedali dei Colli, via san Domenico 96, 80127 Napoli, Italia.

E-mail: adamaff@hotmail.com

Articolo pubblicato secondo la Creative Commons Attribution NonCommercial 4.0 License (CC BY-NC 4.0).

${ }^{\circ}$ Copyright: the Author(s), 2020

Licensee PAGEPress, Italy

QUADERNI - Italian Journal of Medicine 2020; 8(3):64-73 visita collegiale ed i rapporti con l'Ospedale e con il DEA.

Queste aree rappresentano ambiti di miglioramento da considerare nella programmazione delle attività formative ed istituzionali di FADOI e ANÌMO.

La Federazione delle Associazione dei Dirigenti Ospedalieri Internisti (FADOI) e l'Associazione Italiana degli Infermieri di Medicina (ANİMO) nel corso del 2019 hanno effettuato un sondaggio per via telematica (web-based survey) volto a raccogliere ed analizzare le opinioni degli Internisti e degli Infermieri che quotidianamente svolgono la propria attività lavorativa nelle Unità Operative (UO) di Medicina Interna (MI).

La survey denominata Un giorno in Medicina, aveva tra gli scopi quelli di quantificare, decodificare e successivamente analizzare le opinioni e gli orientamenti dei professionisti sanitari su aspetti specifici della realtà ospedaliera utilizzando una serie di item quali: i) gli indicatori più idonei per valutare la qualità dell'assistenza in MI; ii) gli aspetti più soddisfacenti, importanti e peculiari dell'attività di reparto, nonché gli aspetti da modificare; iii) la propensione alla cura dei pazienti critici; iv) i rapporti tra le UO di MI, l'Ospedale e il DEA.

Poiché da sempre FADOI e ANÌMO condividono valori di fondo riguardo la cura della persona ricoverata in MI e ritengono l'interdisciplinarietà la premessa fondamentale alla realizzazione di una presa in carico del paziente e un'assistenza sicura e di qualità si è voluto confrontare i risultati delle due survey con lo scopo di valutare le aree di concordanza o eventuale discordanza nelle opinioni di Medici e Infermieri circa gli aspetti esplorati dalle due survey e ritenuti caratterizzanti l'attività in MI.

Dall'analisi dei dati si possono ricavati spunti per attuare azioni formative, di miglioramento e di discussione societarie e con le istituzioni.

\section{Materiali e Metodi}

I risultati delle due survey sono stati confrontati dopo avere scelto gli item confrontabili. 
I dati sono stati elaborati ed analizzati dal punto di vista statistico dal Centro Studi Fadoi.

\section{Risultati}

Le aree regionali di appartenenza dei soci, i dati generali e le caratteristiche di contesto sono riassunti nelle Tabelle 1 e 2 .

Alla survey hanno aderito 740 Internisti e 408 Infermieri equamente distribuiti su tutto il territorio nazionale categorizzato come nord, centro-nord, centro, sud, isole e sono rappresentativi della quasi totalità della realtà sanitaria della MI italiana.

\section{Questionario A. Indicatori più idonei a valutare la qualità dell'assistenza}

La Tabella 3 presenta la sintesi dei punteggi relativi agli indicatori ritenuti importanti per la qualità delle cure, aggregati per aree tematiche (attività, risorse e organizzazione, processi, esiti). Per entrambi i gruppi (FADOI E ANIMO) il punteggio medio assegnato agli indicatori correlati a risorse/organizzazione (rispettivamente 8,3 e 7,9) e ai processi (rispettivamente 8,3 e 8,4 ) è risultato maggiore di quello riportato dagli indicatori amministrativi correlati all'attività (rispettivamente 7,3 e 7,4) e agli esiti (rispettivamente 7,4 e 7,8).

ANİMO assegna punteggi maggiori alla gestione del fine vita $(8,9$ vs 8,5) e al numero di cadute $(8,0$ vs $7,4)$ mentre FADOI assegna punteggi maggiori alla presenza del Direttore/Coordinatore nelle attività cliniche $(8,1$ vs 7,0$)$ e alla possibilità di eseguire la ventilazione non invasiva (NIV) $(8,3$ vs 8,0$)$.

\section{Questionario B. Aspetti più soddisfacenti, importanti, peculiari e da modificare dell'attività di reparto}

La Tabella 4 riporta i punteggi relativi agli aspetti ritenuti rispettivamente più soddisfacenti, importanti, peculiari e da modificare e le Figure 1-2-3-4 mostrano le maggiori differenze nei punteggi assegnati dai soci delle due società scientifiche.

Il punteggio medio relativo agli aspetti più soddisfacenti è risultato maggiore per FADOI rispetto ad ANÌMO $(8,1$ vs 6,7$)$, mentre è concordante per aspetti importanti $(8,4$ vs 8,1), peculiari $(8,4$ vs 8,2) e da modificare $(8,3$ vs 8,1$)$.

Le due società concordano sull'attribuzione di un punteggio inferiore per l'item visita collegiale alla quale gli Infermieri attribuiscono un punteggio più basso rispetto ai Medici. Anche la collaborazione medico-infermiere, la possibilità di eseguire la NIV e le tecniche strumentali hanno punteggi più alti per FADOI in termini di importanza delle attività rispetto ad ANİMO.

Tabella 1. Risposte per macroarea regionale.

\begin{tabular}{lcc}
\hline & FADOI & ANÌMO \\
\hline Nord & 247 & 207 \\
\hline Centro-Nord & 170 & 75 \\
\hline Centro & 163 & 50 \\
\hline Sud e Isole & 160 & 76 \\
\hline
\end{tabular}

Nord: Piemonte, Liguria, Lombardia, Veneto, Trentino Alto Adige, Friuli Venezia Giulia; Centro Nord: Toscana, Emilia Romagna; Centro: Lazio, Marche, Umbria, Abruzzo, Molise ; Sud e Isole: Campania, Basilicata, Puglia, Calabria, Sicilia, Sardegna.

Tabella 2. Dati generali e di contesto.

\begin{tabular}{|c|c|c|c|c|}
\hline \multirow[b]{2}{*}{ Età partecipanti } & \multicolumn{2}{|c|}{ FADOI } & \multicolumn{2}{|c|}{ ANÌMO } \\
\hline & Totale & $\%$ & Totale & $\%$ \\
\hline$<30$ & 39 & $5,8 \%$ & 32 & $8 \%$ \\
\hline $30-45$ & 295 & $33,9 \%$ & 164 & $40 \%$ \\
\hline $46-55$ & 169 & $22,8 \%$ & 166 & $41 \%$ \\
\hline$\geq 56$ & 237 & $32 \%$ & 46 & $11 \%$ \\
\hline \multicolumn{5}{|c|}{ Ospedale: posti letto } \\
\hline$<200$ & 234 & $31,6 \%$ & 112 & $27 \%$ \\
\hline $200-400$ & 245 & $33,1 \%$ & 149 & $37 \%$ \\
\hline$>400$ & 261 & $35,3 \%$ & 147 & $36 \%$ \\
\hline \multicolumn{5}{|l|}{ Ospedale: sede di } \\
\hline DEA I livello & 258 & $34,9 \%$ & 148 & $36 \%$ \\
\hline$\underline{\text { DEA II livello }}$ & 218 & $29,5 \%$ & 87 & $21 \%$ \\
\hline PS & 205 & $27,7 \%$ & 110 & $27 \%$ \\
\hline Altro & 59 & $7,9 \%$ & 63 & $16 \%$ \\
\hline
\end{tabular}




\section{La cura del paziente critici}

La Figura 5 riporta i dati relativi alla propensione alla cura dei pazienti critici.

La percentuale di Medici ed Infermieri che pensano sia vantaggioso usufruire di posti letto che permettano il monitoraggio dei pazienti è elevata $(93 \% \mathrm{e}$ $92 \%$ rispettivamente) a fronte della limitata disponibilità attuale di questa tecnologia $(<43 \%)$. I professionisti di entrambe le società dichiarano, con punteggi elevati, di essere favorevoli alla gestione dei pazienti critici e disponibili alla formazione in quest'ambito.

\section{I rapporti tra le UO di Medicina Interna, l'Ospedale e il DEA}

Medici $(71,2 \%)$ e Infermieri (73\%) percepiscono come importante e non ancillare, il proprio ruolo all'interno delle proprie $\mathrm{UO}$, ritenute organizzazioni che forniscono una visione forte della MI (54\%), ma dichiarano

Tabella 3. Questionario A. Indicatori di qualità delle cure.

\begin{tabular}{|c|c|c|}
\hline & FADOI & ANÌMO \\
\hline \multicolumn{3}{|l|}{ Indicatori amministrativi correlati all'attività } \\
\hline Numero di re-ricoveri entro un mese & 7,9 & 8,0 \\
\hline Percentuale di pazienti ricoverati dal Pronto Soccorso & 7,5 & 7,5 \\
\hline Percentuale di dimissioni a domicilio & 7,3 & 7,5 \\
\hline Degenza media & 6,9 & 7,3 \\
\hline Numero di pazienti in letti aggiunti in reparto & 7,2 & 7,1 \\
\hline Numero di pazienti appoggiati in altro reparto & 7,2 & 7,1 \\
\hline Numero di dimissioni l'anno & 7,1 & 7,4 \\
\hline Punteggio medio & 7,3 & 7,4 \\
\hline \multicolumn{3}{|l|}{ Indicatori correlati a risorse e organizzazione } \\
\hline Rapporto n. Infermieri/n. pazienti & 8,9 & 9,0 \\
\hline Rapporto n. Medici/n. pazienti & 8,8 & 7,9 \\
\hline Presenza in visita di uno stesso Medico per almeno 5 giorni/settimana & 8,6 & 8,2 \\
\hline Presenza di Direttore/Coordinatore nelle attività cliniche & 8,1 & 7,0 \\
\hline Utilizzo di lettera di dimissione (medico-infermieristica) strutturata & 8,1 & 8,2 \\
\hline Individuazione del Medico che prende in carico il paziente & 7,9 & 8,2 \\
\hline$\underline{\text { Presenza dello stesso Infermiere nella visita quotidiana }}$ & 7,6 & 7,3 \\
\hline Punteggio medio & 8,3 & 7,9 \\
\hline \multicolumn{3}{|l|}{ Indicatori correlati ai processi } \\
\hline Meeting clinici e discussioni collegiali & 8,5 & 8,3 \\
\hline Gestione del fine vita & 8,5 & 8,9 \\
\hline Percorsi diagnostico-terapeutici/assistenziali strutturati e condivisi & 8,4 & 8,3 \\
\hline Organizzazione per continuità assistenziale & 8,2 & 8,6 \\
\hline Possibilità di effettuare esami strumentali ecografici e procedure/tecniche strumentali a scopo assistenziale & 8,4 & 8,3 \\
\hline Possibilità di effettuare la ventilazione non invasiva & 8,3 & 7,8 \\
\hline Gestione del rischio clinico & 8,0 & 8,5 \\
\hline Punteggio medio & 8,3 & 8,4 \\
\hline \multicolumn{3}{|l|}{ Indicatori correlati agli esiti } \\
\hline Grado di soddisfazione dei pazienti & 8,3 & 8,8 \\
\hline Incidenza delle lesioni da pressione & 8,0 & 8,5 \\
\hline Numero delle cadute dei pazienti & 7,4 & 8 \\
\hline Numero dei contenziosi & 7,0 & 7,2 \\
\hline Percentuale di mortalità & 6,9 & 7,2 \\
\hline Percentuale di pazienti sottoposti a cateterismo vescicale & 6,7 & 7,3 \\
\hline Punteggio medio & 7,4 & 7,8 \\
\hline
\end{tabular}


Tabella 4. Questionario B.

\section{Aspetto più soddisfacente}

Rapporto con il paziente

Ripresa risoluzione patologia/autonomia paziente

Rapporti/confronto con i colleghi

Possibilità di svolgere direttamente tecniche strumentali a scopo assistenziale (ecografia per accessi vascolari, cateterismo vescicale, sondino naso-gastrico)

Possibilità di svolgere ventilazione non invasiva

Management clinico-assistenziale

Meeting/riunioni di reparto

Visita collegiale

\section{Aspetto più importante}

Continuità assistenziale

Tempo a disposizione per l'assistenza

Metodo internistico/Pianificazione assistenza personalizzata

Relazione con pazienti/familiari

Collaborazione con altri specialisti/personale di supporto

Collaborazione con i colleghi di reparto

Collaborazione Medico/Infermiere

Percorsi predefiniti per il trasferimento pazienti

Possibilità di svolgere direttamente tecniche strumentali a scopo assistenziale

(accessi vascolari, cateterismo vescicale, sondino naso gastrico)

Posibilità di svolgere la ventilazione non invasiva

Visita collegiale

\section{Aspetto peculiare}

Visione globale

Pianificazione assistenziale

Valutazione priorità terapeutiche/assistenziali

Comunicazione pazienti/familiari

Diagnosi difficili/Elevata complessità assistenziale

Visita collegiale

\section{- \\ Aspetto da modificare}

Eccesso di pazienti in carico

Organizzazione lavoro e turni

Dotazione organica

$\begin{array}{rr} & 8,3 \\ 8,0 \\ 7,4\end{array}$

Punteggio medio

8,4

8,4

FADOI

ANÌMO

P value

8,9

8,3

7,8

7,4

8,4

7,0

$8,1 \quad 6,9$

$8,1 \quad 6,5$

$7,8 \quad 6,1$

Punteggio medio

7,3

5,6

8,1

6,7

6,9
6,5
6,4
6,1
5,6
6,7


che poco frequentemente gli atti e le decisioni della Direzione Strategica/Sanitaria tengano in giusta considerazione l'attività da loro svolta $(24,4-28,0)$.

Solo il 45\% degli Internisti e $32 \%$ degli Infermieri dichiarano di essere coinvolti "spesso" nell'elaborazione di percorsi/protocolli aziendali (Figura 6).

Il percorso di condivisione con il Pronto Soccorso per la definizione del flusso dei ricoveri o la consulenza strutturata nelle emergenze/urgenze è ritenuto importante dal 95\% dei Medici e dal 98\% degli Infermieri che considerano i ricoveri appropriati nel 57\% e $46 \%$ dei casi rispettivamente (Figura 7).

\section{Discussione}

Questa survey per la prima volta raccoglie e confronta le opinioni di Medici ed Infermieri delle MI relativamente ad alcuni aspetti organizzativi e valoriali che non possono essere dati scontati in un contesto assistenziale complesso in cui l'interdisciplinarietà assume connotati rilevanti.

La ricerca di analogie e differenze nelle risposte ha lo scopo di dare forza agli aspetti che caratterizzano l'attività nelle MI e di individuare possibili ambiti di riflessione e miglioramento.

Per quanto riguarda la qualità delle cure, i soci FADOI e ANÌMO esprimono opinioni concordanti dando maggiore importanza agli indicatori correlati alle risorse/organizzazione e ai processi piuttosto che ai fattori amministrativi correlati alle attività e agli esiti.

Questo è in linea con la letteratura che, se da un lato mette in discussione la validità degli abituali indicatori della qualità, dall'altro evidenzia la differente visione della sanità per mentalità e formazione tra $\mathrm{i}$ gestori e i professionisti, questi ultimi identificandosi con la propria professione, la cura e l'organizzazione vogliono vedere riconosciuta la propria autonomia da parte del management. ${ }^{1,2}$

Sorprende tuttavia i minor peso dato agli esiti. Questo può essere dovuto al limitato ventaglio di item proposti dalla survey, infatti nell'indagine ANÌMO, che ne conteneva un numero maggiore, il punteggio era più elevato $(8,1)$ e sicuramente per i Medici gli item proposti dalla survey non descrivono del tutto la vasta gamma di esiti sensibili all'attività clinica.

ANİMO assegna punteggi maggiori al numero di cadute $(8,0$ vs 7,4), ciò probabilmente è dovuto al fatto che culturalmente gli Infermieri si sentono maggiormente responsabili di questo evento avverso annoverato comunque tra gli outcome relativi all'organizzazione. ${ }^{3}$

Anche la gestione del fine vita presenta un peso diverso tra le due associazioni $(8,9 v s 8,5)$ che da tempo si adoperano per diffondere la cultura della corretta gestione della persona in fine vita ricoverata in ospedale. FADOI nel 2017 ha dedicato un'intera monografia alle problematiche di fine vita ${ }^{4}$ e da quattro anni con ANİMO organizza una giornata di discussione sul questo tema che vede una particolare sensibilità degli Infermieri a cogliere i bisogni del paziente morente rispetto ai Medici. Questi essendo più coinvolti nella diagnosi e nella responsabilità decisionale possono essere trattenuti dalle incertezze esistenti, con il peso

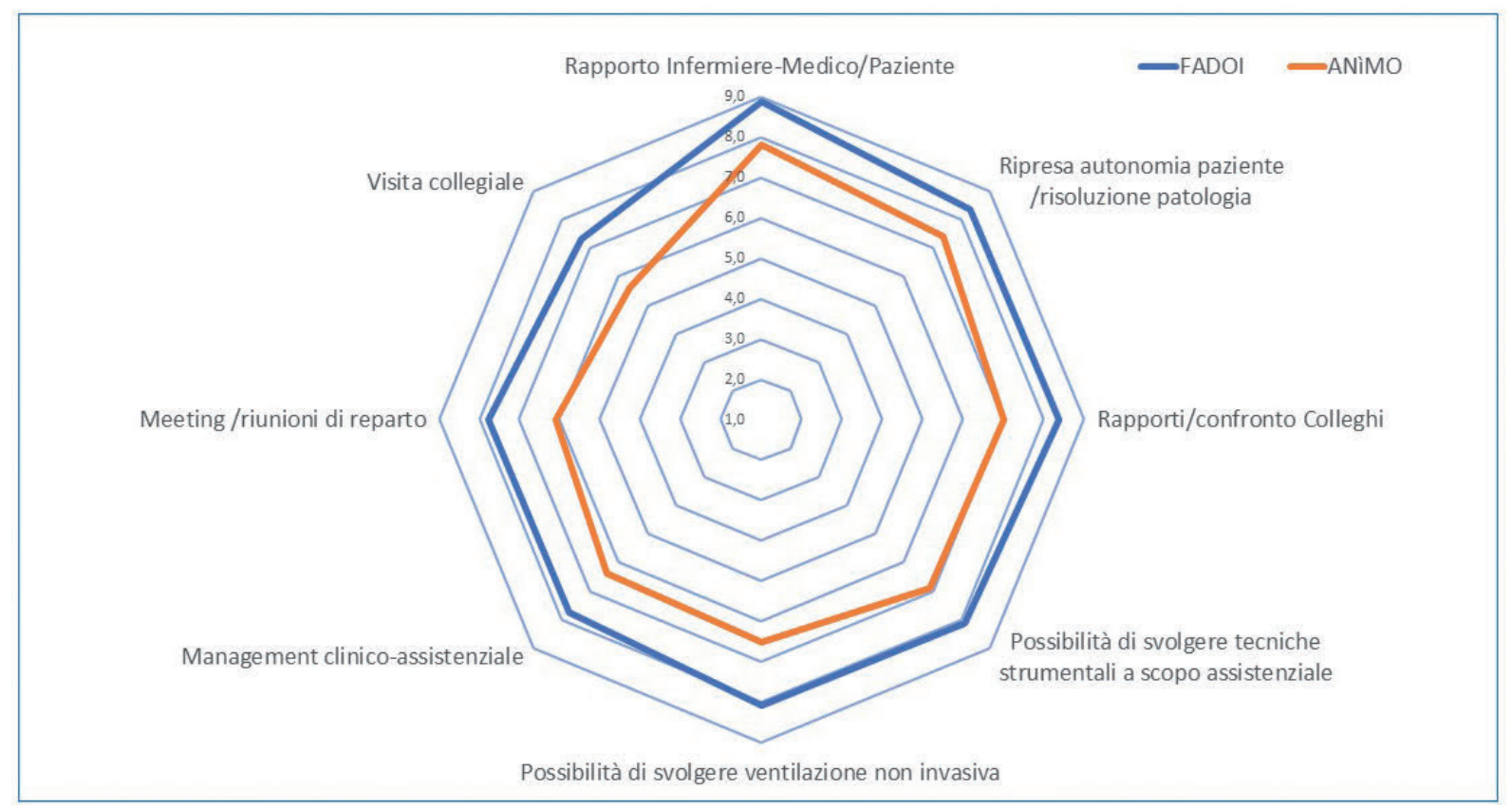

Figura 1. Aspetti più soddisfacenti dell'attività. I punteggi di ANÌMO sono inferiori rispetto a FADOI; il punteggio più basso è assegnato alla visita collegiale. 
delle tendenze conservatrici e degli atteggiamenti della medicina difensiva che si traducono in una certa resistenza al cambiamento. ${ }^{5}$

La presenza del Direttore/Coordinatore nelle attività cliniche assume per gli Infermieri un punteggio più basso $(7,0)$ rispetto a quello attribuito dai Medici $(8,1)$. La motivazione potrebbe essere collegata al fatto che la figura del Coordinatore negli anni si è sempre più staccata dalla clinica, in quanto i percorsi formativi e le richieste aziendali lo hanno portato ad assumere un ruolo più manageriale ed organizzativo. ${ }^{6}$

Sia i Medici che gli Infermieri, per garantire la qualità delle cure, considerano di estrema validità il rapporto numerico Infermiere/pazienti, ma altrettanto non avviene per gli Infermieri che assegnano al rapporto Medico/pazienti un punteggio più basso.

Relativamente agli aspetti dell'attività più soddisfacenti, più importanti, peculiari e da modificare appare

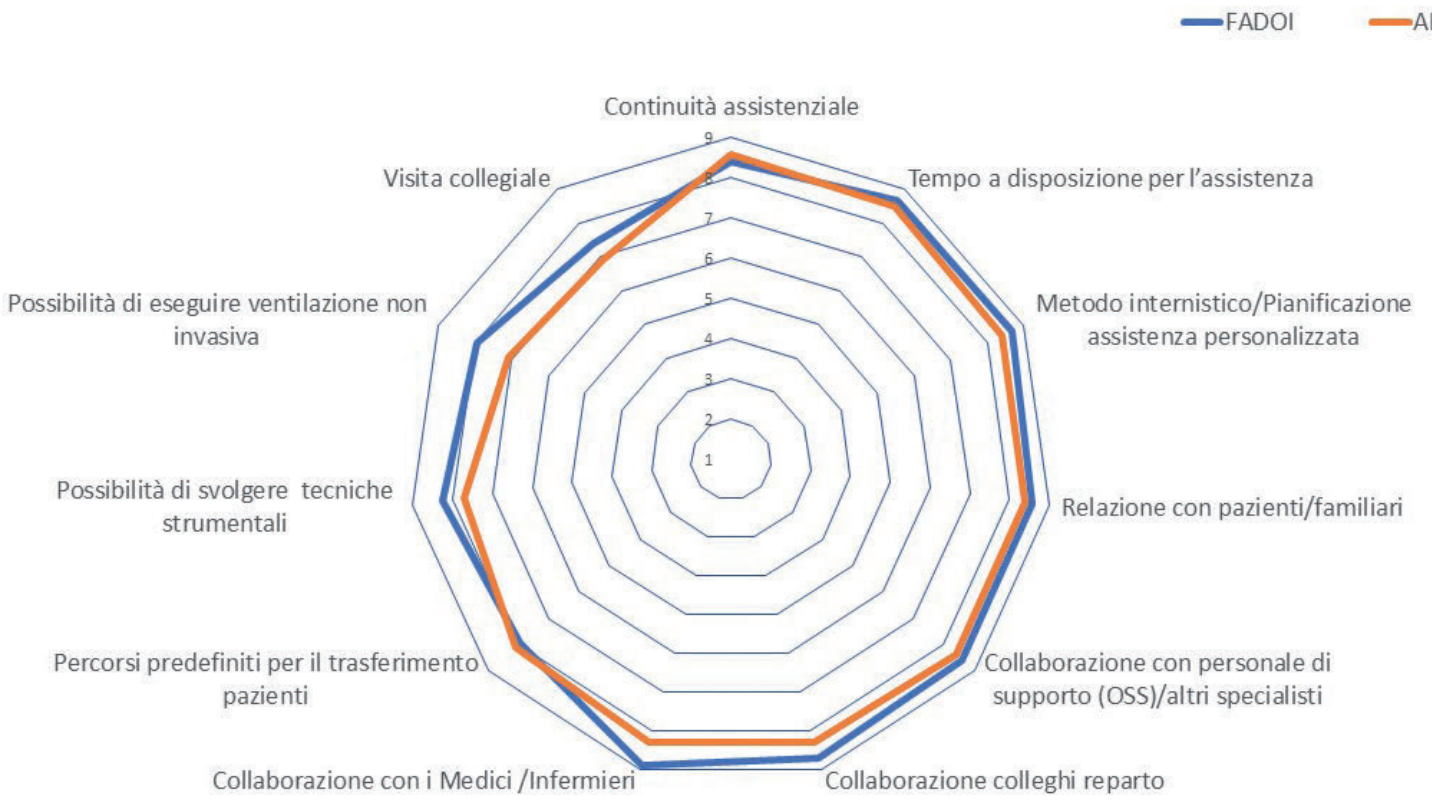

Figura 2. Aspetti più importanti dell'attività. I punteggi di ANÌMO sono inferiori rispetto a FADOI sugli item visita collegiale, ventilazione non invasiva e possibilità di svolgere tecniche strumentali.

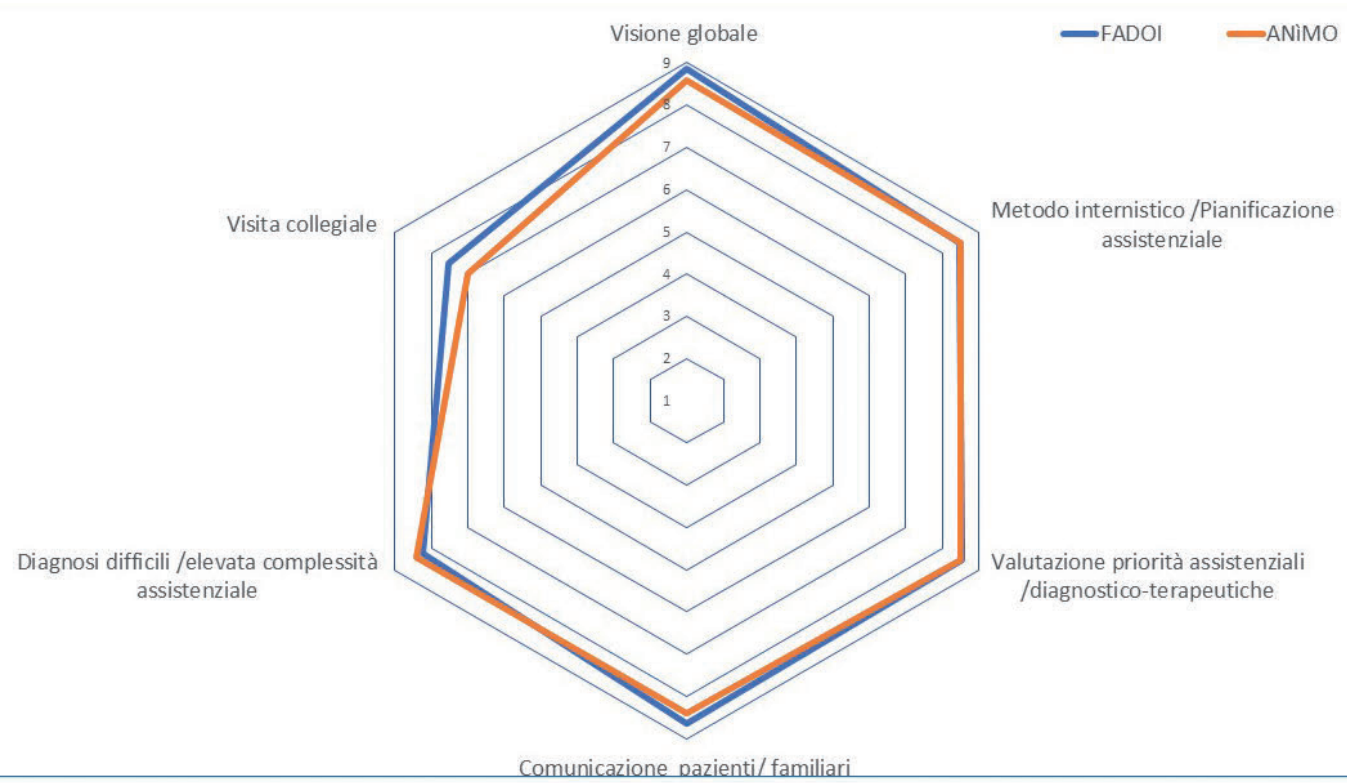

Figura 3. Aspetti peculiari dell'attività. I punteggi di ANÌMO sono inferiori rispetto a FADOI sull'item visita collegiale. 
evidente che gli item relativi agli aspetti che indagano la soddisfazione hanno per ANIMOO complessivamente dei punteggi più bassi. Probabilmente i fattori considerati non sono i più idonei ed esaustivi ad indagare il fenomeno di per sé complesso ma che potrebbe essere considerato come ambito per una valutazione più approfondita. L'elevato turn-over del personale che lavora nelle MI riconosce le sue radici anche nel moral-distress e nell'insoddisfazione lavorativa che deriva dal livello di staffing, dalle caratteristiche dell'ambiente di lavoro, e dall'insieme di esiti infermieristici tra cui le cure non erogate per mancanza di tempo (missed care). Questi fattori possono portare gli Infermieri al burn-out e ad esprimere la volontà di cambiare reparto o di lasciare la professione..$^{7-9}$

Gli aspetti importanti, peculiari dell'attività sono per lo più condivisi dai due gruppi professionali. Estremamente interessante l'importanza che viene data alla visione globale, alla gestione della complessità, alla collaborazione in team e alla comunicazione

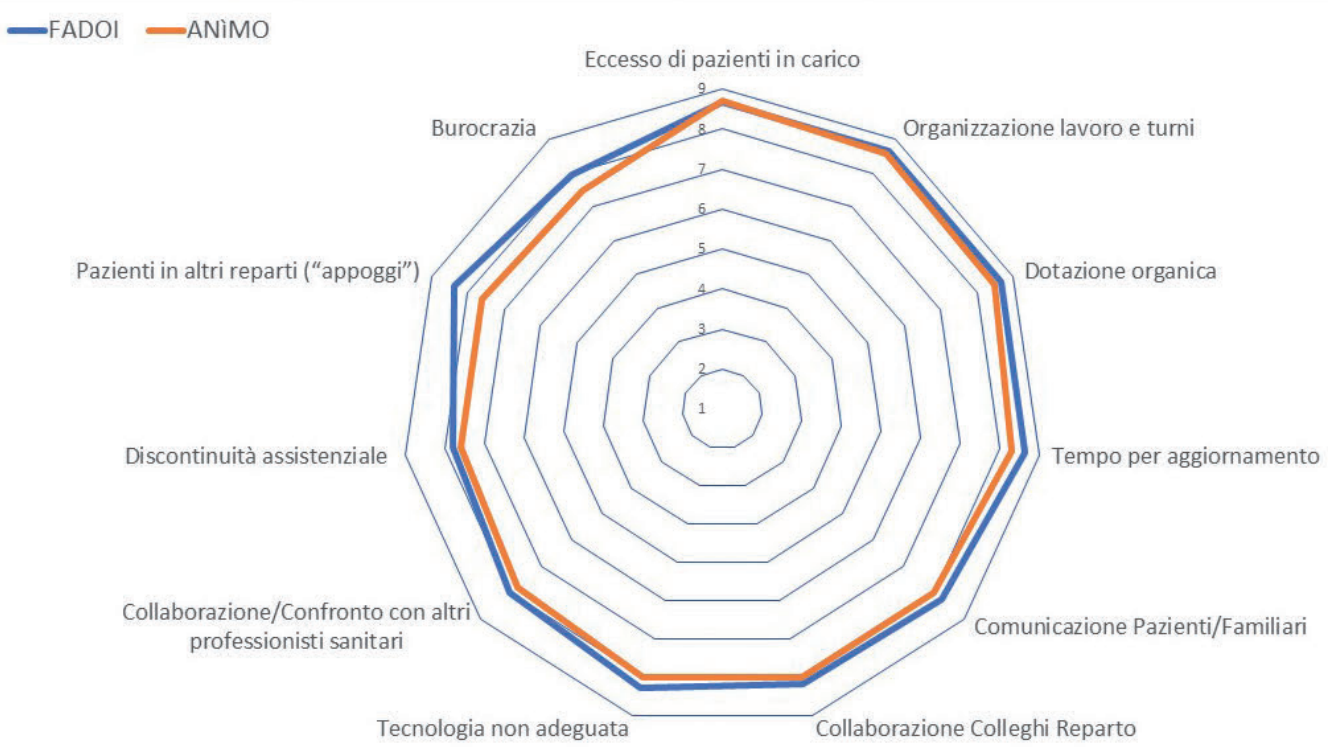

Figura 4. Aspetti da modificare.

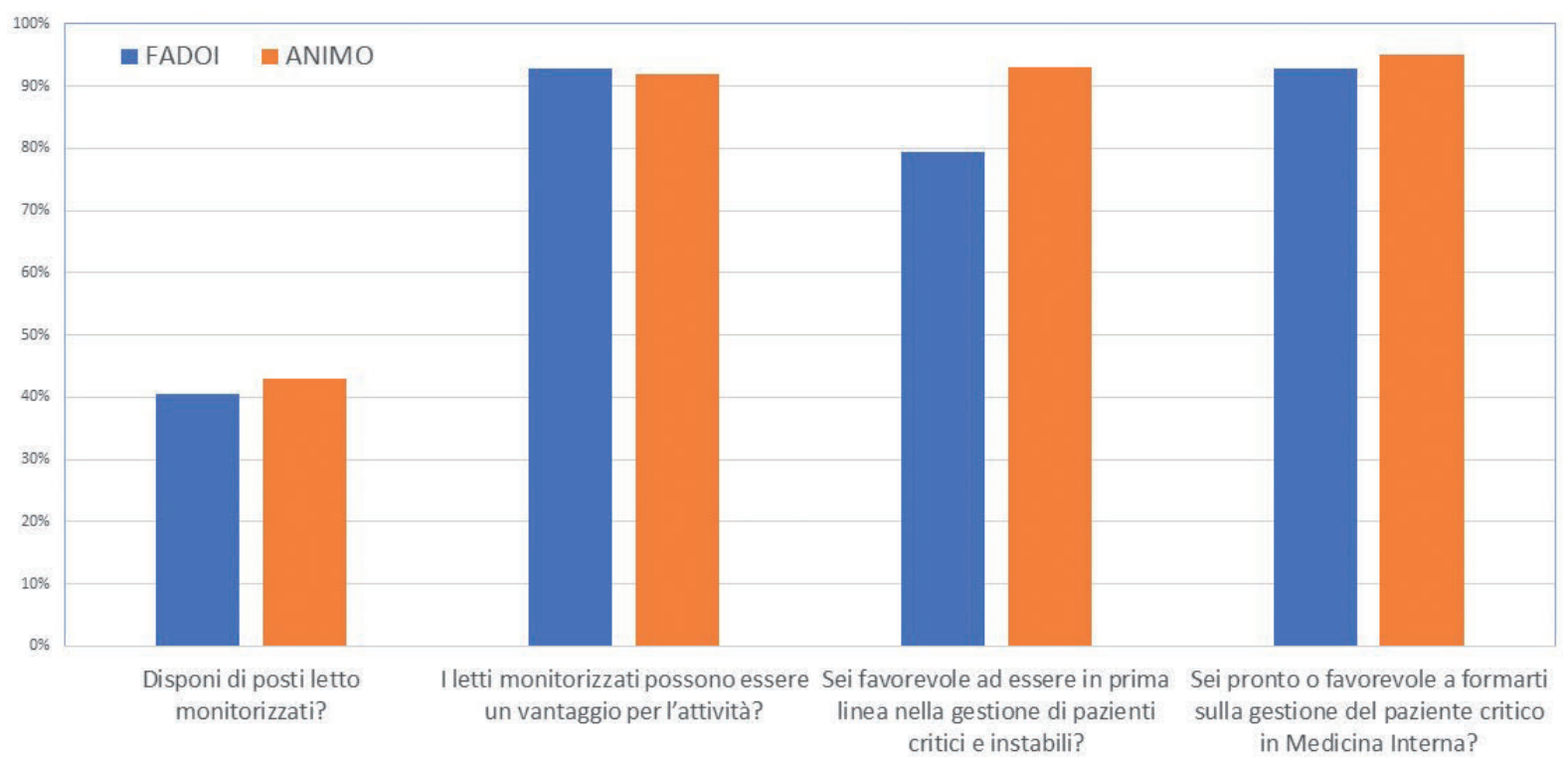

Figura 5. Propensione alla cura dei pazienti critici. 
con i pazienti e familiari. Chi lavora in MI deve essere in grado di dare risposte alle sempre più complesse e articolate esigenze dell'utente, diventa quindi fondamentale creare quel clima di empatia utile al buon esito delle cure e necessario a favorire al meglio l'integrazione tra professionisti. ${ }^{10}$

Alcune divergenze di opinione si notano per la possibilità di eseguire procedure a scopo diagnostico/assistenziali, la NIV e per il giro visita $(\mathrm{GV})$ che hanno punteggi minori per ANÌMO in termini di importanza e peculiarità.

Per quanto riguarda la possibilità di eseguire procedure e NIV, non sorprende che il punteggio degli Infermieri sia più basso. Pur riconoscendo l'importanza della tecnologia, gli Infermieri sono più orientati ad aspetti distintivi della professione quali la ripresa
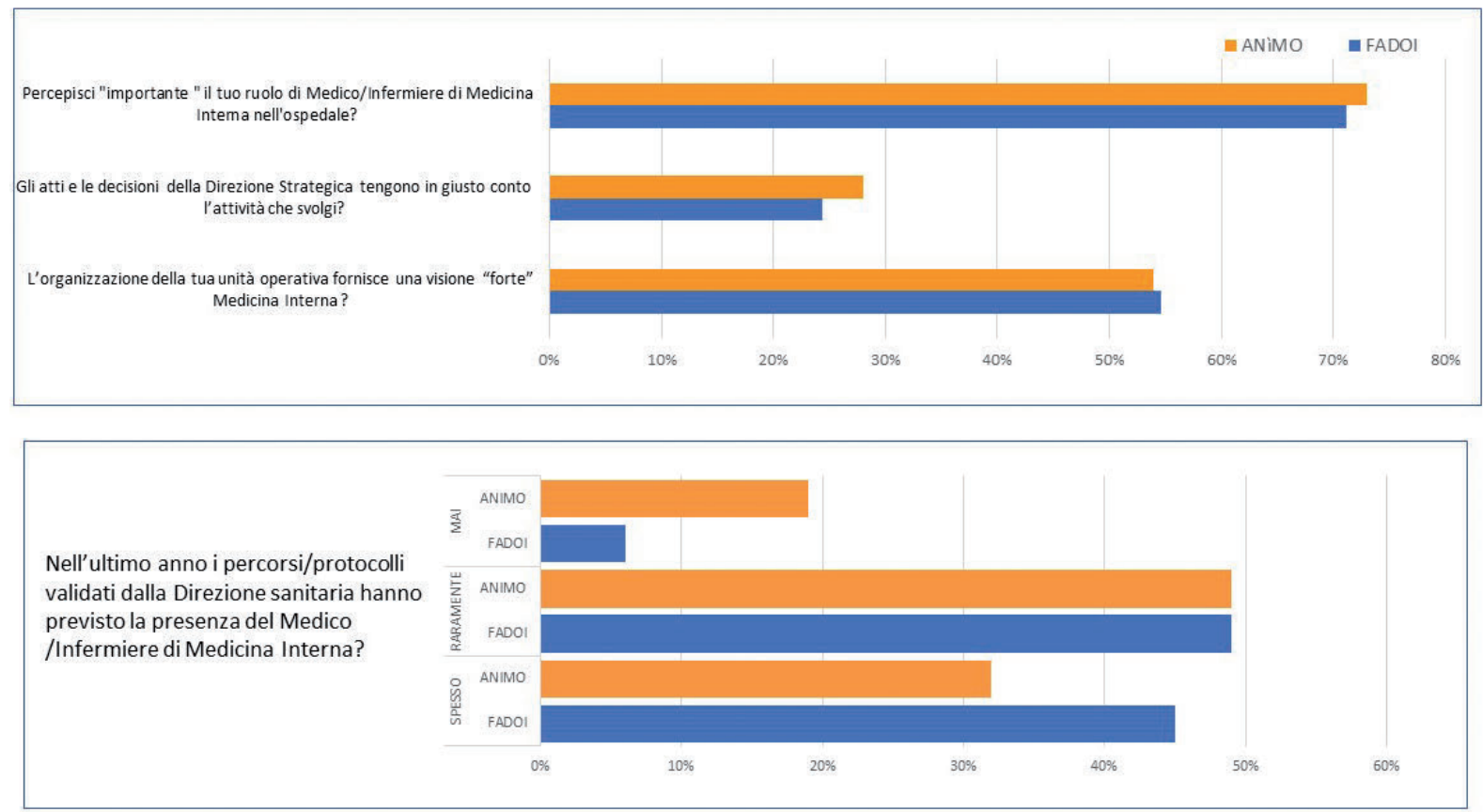

Figura 6. Rapporto con l'ospedale.

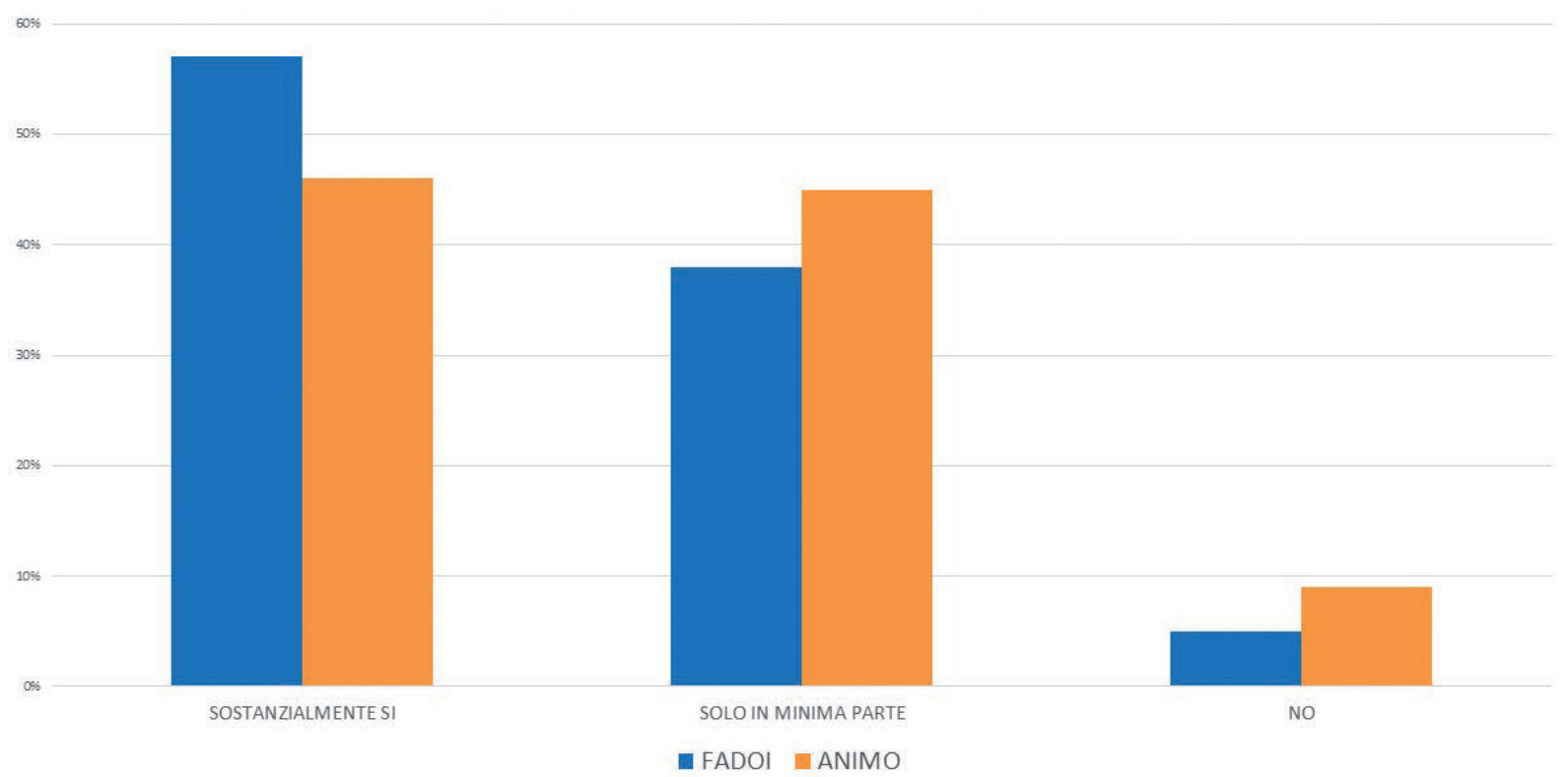

Figura 7. Rapporti tra le Unità Operative di Medicina Interna, l'Ospedale e il Dipartimento Emergenza e Accettazione. 
dell'autonomia della persona, la relazione con l'assistito, la collaborazione interna all'equipe, la pianificazione assistenziale, mentre merita una riflessione particolare il basso peso dato alla visita collegiale.

Come evidenziato da una recente revisione della letteratura su questo tema, il GV rappresenta un importante strumento di comunicazione, attraverso il quale i professionisti sanitari si scambiano informazioni al fine di un proficuo coordinamento delle cure e dell'assistenza. Dalla disamina della letteratura si evince come durante il GV la figura del Medico a volte prevalga su quella degli Infermieri determinando outcome negativi come la scarsa soddisfazione professionale ed il limitato coinvolgimento nella presa di decisioni clinico-assistenziali. D'altra parte un'ulteriore considerazione che può spiegare il limitato coinvolgimento degli Infermieri all'interno del GV è la scarsa consapevolezza del proprio ruolo all'interno del team $^{11} \mathrm{e}$ della responsabilità dell'assistenza che è e resta a loro carico.

Relativamente agli item che indagano i rapporti con l'Ospedale, entrambe le categorie di professionisti sono unanimi nel riconoscere come forte la visione della MI nella propria realtà lavorativa, così come entrambe ritengono importante il proprio ruolo svolto all'interno dell'ospedale come rappresentanti di uno snodo chiave rappresentato dalla MI.

Prevale la percezione per entrambi i gruppi di come gli atti e le decisioni della Direzione Strategica non tengano in giusto conto l'attività svolta.

Alla domanda se nell'ultimo anno i percorsi/protocolli validati dalla Direzione Sanitaria abbiano previsto la presenza dell'Internista o dell'Infermiere di MI, quasi il 50\% di entrambe le categorie ha risposto raramente. Appare quindi chiaro lo scollamento tra la evidente importanza del percorso clinico assistenziale della MI e nel contempo della relativamente scarsa considerazione che la stessa ed i suoi operatori hanno all'interno delle strategie aziendali.

La propensione ad assistere pazienti critici e la disponibilità ad essere formati in quast'area manifesta la necessità di acquisire competenza nell'assistere questi pazienti sempre più frequesti in MI. Circa il 10 $\%$ dei pazienti ricoverati in MI ha necessità di un setting assistenziale che garantisca trattamenti di alta intensità e necessità di monitoraggio specifico. ${ }^{12}$ Nonostante ciò le UO dotate di posti letto monitorizzati sono limitate a questo evidenzia la necessità che si renda disponibile una tecnologia adeguata a supporto dell'attività clinica.

In quest'ottica il rapporto con il DEA diventa fondamentale in termini di percorsi condivisi, modalità comunicative adeguate, attenzione all'appropriatezza dei ricoveri come testimoniato dalla quasi totalità delle risposte date dai partecipanti.

Le criticità del contesto sono vissute in modo uni- voco da Medici ed Infermieri, infatti la dotazione dell'organico, l'eccessivo carico di lavoro, l'organizzazione di lavoro ed i turni orientati all'efficientismo, la burocrazia, i tempi inadeguati per la formazione e la relazione con l'assistito e i colleghi sono gli aspetti da modificare per i quali le opinioni delle due associazioni sono sovrapponibili.

Il problema degli appoggi è più sentito dai medici che frequentemente, ad iso-risorse, devono spostarsi in altri setting, generalmente chirurgici, per seguire malati affidati a una equipe infermieristica che ha meno familiarità nella gestione dei casi clinici tipici della MI. Questo fenomeno, conseguenza dei tagli ai posti letto ed espressione di un mancato allineamento fra cambiamenti demografici e organizzazione ospedaliera, rappresenta una seria minaccia alla qualità e alla sicurezza delle cure erogate dai reparti di MI ed ha ricadute negative sulla soddisfazione di pazienti e operatori sanitari. ${ }^{13}$

\section{Conclusioni}

Complessivamente questa survey, pur con i limiti legati al tipo di indagine, evidenzia molti punti in comune tra le opinioni dei Medici e degli Infermieri delle MI.

Entrambi i gruppi professionali si esprimono a favore degli indicatori associati alle risorse e alla organizzazione delle attività cliniche piuttosto che verso gli indicatori di carattere prevalentemente amministrativo di solito proposti dai decisori istituzionali delle politiche sanitarie.

I dati confermano l'importanza data da FADOI e ANİMO alla cura della persona in primis e della complessità clinica che conservano appieno la loro validità anche nell'ambito di uno scenario sanitario che sta modificandosi e per il quale diventano prioritarie la continuità assistenziale, la comunicazione, la cultura del fine vita e la collaborazione interdisciplinare.

Ribadiscono le distanze spesso presenti con l'Amministrazione e con la Direzione Sanitaria e la necessità di percorsi strutturati ed in collaborazione con il DEA in un'ottica di continuità assistenziale, di comunicazione e collaborazione con tutti gli attori della vita ospedaliera.

La survey mostra la chiara propensione all'ampliamento delle competenze soprattutto per la cura del paziente critico e per la necessaria formazione che è auspicata da entrambi i gruppi.

Si notano alcune divergenze di opinione per la possibilità di eseguire procedure a scopo diagnostico/assistenziali e la NIV che sono ritenute meno importanti per gli Infermieri rispetto ad elementi quali la continuità assistenziale, la cultura del fine vita, la comunicazione ed il lavoro interdisciplinare.

Il consenso si esprime anche nel riconoscere gli 
aspetti da modificare quali carico di lavoro eccessivi, modelli organizzativi orientati all'efficientismo, burocrazia, tempi inadeguati per la formazione e la relazione con l'assistito e i colleghi.

Altre criticità comuni sono costituite dai rapporti con le Direzioni Strategiche e il DEA. Infatti, le risposte ad entrambe le survey evidenziano la distanza significativa tra gli atti delle Direzioni Sanitarie e i professionisti nella percezione dell'importanza strategica dell'attività complessiva svolta dalle M.I.

Il minor grado di soddisfazione e importanza espresso da entrambi i gruppi nei confronti della visita collegiale, più accentuata per gli Infermieri, merita una discussione interna e condivisa tra FADOI e ANÌMO. È necessario rivedere le modalità di realizzazione di questa attività fondamentale e caratterizzante la MI, rafforzando la comprensione dei ruoli e delle responsabilità di ciascun professionista, elementi che già caratterizzano la vision delle due Società scientifiche.

I risultati della survey definiscono aree tematiche che FADOI e ANİMO devono considerare nella programmazione di attività formative orientate all'interdisciplinarietà e di confronto politico-istituzionale per definire iniziative di miglioramento del contesto delle MI.

\section{Ringraziamenti}

Annachiara Crespi, Clinical Project Manager, Centro Studi FADOI.

\section{Bibliografia}

1. De Carli S. È ancora possibile una reale alleanza fra clinici e gestori per una Medicina Interna ospedaliera sostenibile? Quaderni Ital J Med 2014;2:95-100.

2. Foglia E, Croce D. Indicatori della qualità nella Medi- cina Interna Ospedaliera: solamente durata della degenza, ricoveri inappropriati, mortalità intraospedaliera e tasso di re-ricovero a 30 giorni? Quaderni Ital J Med 2014;2:106-13.

3. Doran DM, Pringle D. Gli outcome dei pazienti come responsabilità diretta. In: Doran DM. Nursing outcome. Gli esiti sensibili nelle cure infermieristiche. Milano: MC Graw Hill; 2013. pp 1-20.

4. Zulian F, Campanini M, Lusiani L, et al. Problematiche di fine vita: il ruolo della medicina interna ospedaliera. Quaderni Ital J Med 2017;5(1).

5. Lusiani L, Bullo C. Il concetto di terminalità: certezze e incertezze. Quaderni Ital J Med 2017;5(1):23-7.

6. Sarli P. Il coordinatore nell'organizzazione che cambia: scenari possibili. Atti XIII Congresso Nazionale CNC Parma 2019.

7. Sasso L, Bagnasco A, Catania G, et al. Il numero di pazienti assistiti da ogni infermiere, le cure mancate, la qualità dell'assistenza e la sicurezza dei pazienti. Alcuni dati italiani dello studio RN4CAST per una riflessione condivisa. L'infermiere 2019;56:3:43-50.

8. Morley G. What is "moral distress" in nursing? How, can and should we respond to it? J Clin Nurs 2018;27:3443-5.

9. Sasso L, Bagnasco A, Catania G, et al. Push and pull factors of nurses'intention to leave. J Nurs Manag 2019;27:946-54.

10. Lenti S, Felici M, Campanini M, et al. La comunicazione con il paziente e tra professionisti nella gestione del paziente complesso in medicina interna. Quaderni Ital Med 2017;5(1):1-81.

11. Simonetti V, Comparcini D, Tesei L, et al. Il giro visita: una prassi che garantisce la qualità? Una revisione integrativa della letteratura. L'infermiere 2019;56:e106-19.

12. Bartolomei C, Cei M. L'allocazione dei pazienti in un reparto di Medicina Interna organizzato per intensità di cure: lo studio ADOIT Tri-Co (Triage di Corridoio) Ital J Med 2007;2:31-9.

13. La Regina M, Guarneri F, Bellandi T, et al. Gestione ricoveri in appoggio e rischio clinico Quaderni Ital J Med 2019;7(6):105-10. 


\title{
Il core competence dell'infermiere in Medicina Interna: evoluzione e opportunità
}

\author{
Michela Piasentin, Fabio Bertoncini \\ Gruppo Nazionale Ricerca Associazione A.N.ì.M.O.; ASL Biella, Italia
}

\section{Core competence}

Di recente l'interesse politico sull'impatto che le competenze potrebbero avere sui servizi sanitari è crescente e questo sta determinando un cambiamento nelle strategie sanitarie verso un'assegnazione delle risorse umane più efficace ed efficiente. Questa necessità di cambiamento è dettata da una serie di cause che interessano le riforme dei servizi sanitari, i problemi di budget, di assunzione e fidelizzazione del personale, causati dalla carenza di professionisti sanitari, parallelamente all'affacciarsi di cambiamenti demografici, quali l'aumento dell'invecchiamento della popolazione globale. Sebbene vi sia molta attenzione, sia politica che sanitaria, rispetto al concetto di competenza, la letteratura dimostra che, in realtà, esso non è chiaramente definito, e che questa condizione sta producendo una serie di controversie e discussioni circa l'autonomia professionale e i confini dei vari operatori sanitari, con conseguenti potenziali conflitti tra $\mathrm{i}$ gruppi professionali. ${ }^{1}$

Uno dei principi cardine in cui i professionisti sanitari sono chiamati oggi a mantenere un elevato livello di qualità, risulta essere il mantenimento di un focus costante sull'erogazione di un'assistenza basata su evidenze provenienti da informazioni aggiornate, in linea con lo sviluppo del progresso scientifico e tecnologico. ${ }^{2}$

I professionisti sanitari lavorano in ambienti a rapida evoluzione a causa dei processi di innovazione tecnologica e dei progressi scientifici, ${ }^{3}$ per questo diventa

Corrispondente: Michela Piasentin, Gruppo Nazionale Ricerca Associazione A.N.ì.M.O.; ASL Biella, Italia.

E-mail: michela.piasentin@aslbi.piemonte.it

Articolo pubblicato secondo la Creative Commons Attribution NonCommercial 4.0 License (CC BY-NC 4.0).

${ }^{\circ}$ Copyright: the Author(s), 2020

Licensee PAGEPress, Italy

QUADERNI - Italian Journal of Medicine 2020; 8(3):74-77 quindi fondamentale modificare la propria pratica, mantenendo aggiornate le competenze da agire in funzione delle sollecitazioni e alle esigenze dei sistemi in cui operano e delle popolazioni a cui si rivolgono. ${ }^{4}$

Il cambiamento è costante in tutti gli standard di pratica infermieristica e sanitaria e l'implementazione di nuove pratiche richiede oggi infermieri in grado di dimostrare competenza nella pratica clinica specialistica, poiché la qualità delle cure può essere realizzata solo se gli infermieri sono ritenuti competenti per fornire il miglior standard di cura. ${ }^{5}$

Sebbene le pratiche innovative non siano generalmente apprese nei programmi educativi formali in quanto scaturiscono dall'evoluzione dei tempi, per un'assistenza sanitaria innovativa, gli infermieri ricoprono un ruolo importante. ${ }^{6}$

La letteratura è coerente nell'affermare che la formazione di base non permette di mantenere e sostenere le competenze e le conoscenze acquisite per tutta la durata della carriera lavorativa di un professionista. ${ }^{2}$

Tutti i professionisti della salute, infatti, dovrebbero partecipare o dedicare regolarmente parte del loro tempo ad attività di aggiornamento continuo, con lo scopo di migliorare e ottimizzare le cure e, soprattutto, gli esiti sulla persona assistita. ${ }^{7}$

In passato, per mantenere attuale un aggiornamento professionale e uno sviluppo delle conoscenze evidence-based, era sufficiente consultare libri di testo, articoli di giornale o il confronto con altri colleghi.?

Con l'avanzare della tecnologia, le opportunità di formazione online sono diventate sempre più diffuse $\mathrm{e}$ utilizzate, ${ }^{8}$ tanto che, oggi, il web risulta essere lo strumento più utilizzato per accedere alla conoscenza, ${ }^{7}$ rendendo però difficoltoso e quasi impossibile il controllo, in termini di validità metodologica delle informazioni cui si accede $\mathrm{e}$ facendo così emergere numerose criticità correlate all'evidence-based practice. ${ }^{9}$

I principali ostacoli che gli infermieri descrivono nei confronti di questo tipo di approccio sembrano essere correlati alla scarsità di tempo, alla mancanza di esperienza e di conoscenze legate a questo processo ${ }^{10} \mathrm{e}$ al cospicuo numero di risultati derivanti dalla ricerca. ${ }^{11}$

Altre cause che risultano essere in grado di influenzare l'utilizzo delle conoscenze provenienti dalla 
ricerca hanno come determinante comune proprio la metodologia: molti articoli sono troppo tecnici, contengono analisi statistiche complesse che i fruitori non sempre comprendono e sono scritti in uno stile accademico non facile da leggere. ${ }^{10}$

La letteratura è concorde nell'affermare che per garantire uno standard di competenza adeguato è necessario che gli infermieri ricorrano all'aggiornamento continuo delle loro conoscenze. ${ }^{12}$

Nell'ottica dello sviluppo delle risorse umane valutare le competenze e promuovere l'empowerment professionale diviene un elemento fondamentale per qualsiasi organizzazione. La competenza infermieristica è fondamentale per un'assistenza di qualità. ${ }^{13}$

Secondo Levati, la competenza è una caratteristica intrinseca di un individuo, appartenente alla dimensione psicologica, costituita dall'insieme di capacità, conoscenze, esperienze finalizzate. Si esprime attraverso comportamenti e necessita, per esprimersi, dell'azione di motivazione e di contesto. L'aspetto specifico del contesto, che impatta sulla nascita e sviluppo della competenza, è la cultura organizzativa. L'azione combinata di motivazione $e$ contesto fanno assumere alla competenza il suo carattere soggettivo di consapevolezza, di possibilità di controllo sull'ambiente esterno (padronanza). ${ }^{14}$

$\mathrm{Al}$ fine di mantenere aggiornate competenze di natura clinico-specialistica, il solo ricorso alle banche dati o alle evidenze disponibili, però, non è sufficiente o facilmente praticabile: nell'ultimo decennio, la letteratura segnala come vi sia stato un sovraccarico di produzioni scientifiche, con oltre un milione di pubblicazioni annue su PubMed e, per contro, come in alcuni ambiti di ricerca rimanga una importante carenza di prove di efficacia. ${ }^{15}$

Il panorama internazionale e la letteratura sono concordi nell'affermare che la priorità, è quella di velocizzare e ottimizzare il processo di trasferimento alla pratica delle nuove conoscenze, con lo scopo di migliorare gli esiti e ridurre i trattamenti inappropriati e potenzialmente dannosi. ${ }^{16}$

In un'epoca in cui la richiesta di responsabilità nel settore sanitario è in crescita, diventa quindi fondamentale comprendere come gli infermieri sviluppano le loro competenze, aumentano le loro conoscenze. ${ }^{13}$

Sebbene non esista una definizione universale delle competenze infermieristiche, è noto che un professionista esperto possiede conoscenze e abilità avanzate. ${ }^{13}$

La competenza è una misura della prestazione che è l'espressione attiva e comportamentale dell'esperienza che giace su un continuum dal principiante all'esperto. Gli alti livelli di competenza non sono garantiti solo con l'esperienza. ${ }^{13}$

Come già descritto da Levati, ${ }^{14}$ per competenza s'intende un sistema di capacità, conoscenze ed esperienze finalizzate, mentre i comportamenti diventano prestazioni efficaci se sono coniugate in maniera congruente con il ruolo organizzativo: la capacità caratterizza la parte stabile delle competenze, mentre le conoscenze ed esperienze finalizzate sono la parte flessibile, incrementabile e acquisibile.

Le competenze possono rimanere a livello potenziale senza l'intervento del contesto e della motivazione.

Nello studio di Cunningham, viene citato il lavoro filosofico di Drummond sull'economia della conoscenza, ritenuto molto utile quando applicato alla concettualizzazione del mix di abilità correlato ai fattori contestuali. Come descritto, i fattori contestuali agiscono a più livelli, tra cui il livello strutturale, organizzativo e di reparto. A livello di reparto, le modifiche al mix di abilità sono spesso influenzate dalle esigenze del paziente. ${ }^{1}$

Nell'erogazione dell'assistenza, l'incremento di malattie croniche, tipiche dei setting di area medica, ha sostenuto un cambiamento culturale un tempo fondato sulla gestione dell'acuzia, oggi sull'individuazione di strategie in grado di far fronte alla complessità della cronicità.

Il Piano Nazionale Cronicità pubblicato dal Ministero della Salute nel 2016 accompagna e sostiene i professionisti all'erogazione di interventi personalizzati attraverso il piano di cura, fondato su tre principi fondamentali: assistenza centrata sulla persona, $\mathrm{em}$ powerment e auto-cura. ${ }^{15-17}$

La cura della persona basata sulla centralità della presa in carico dovrà seguire percorsi integrati, organizzati per processo, che intersecano le diverse facilities, interagendo anche con i processi di ricerca per favorire il trasferimento immediato delle scoperte scientifiche alla clinica.

Porre al centro del processo di cura la persona assistita, con la sua unicità e specificità, è il principio cardine, il valore su cui basare le politiche, i principi e i processi di organizzazione e gestione clinica. ${ }^{18}$

In quest'ottica e in questo contesto culturale, emerge anche la necessità di sviluppare un'assistenza sanitaria culturalmente competente. L'infermiere si trova spesso ad assistere persone e famiglie che hanno culture diverse e che a volte ne influenzano la percezione di salute e le diverse manifestazioni di bisogno o più semplicemente di comprensione e adesione ai regimi terapeutici. I professionisti che non posseggono uno standard minimo di esperienza e conoscenze adeguate a essere culturalmente competenti determinano la qualità delle prestazioni erogate. ${ }^{19}$

La rapidità con cui emergono e approdano le innovazioni e i cambiamenti richiede un rapido processo di trasferimento delle evidenze all'applicazione pratica. ${ }^{18}$

Tale rapidità non permette di attendere la fine del processo cognitivo per iniziare quello applicativo e formativo. ${ }^{18}$

Ai fini dell'apprendimento organizzativo è interes- 
sante sottolineare la costante interdipendenza tra persona e gruppo in cui è inserita e quanto quest'ultimo incida sullo sviluppo delle competenze individuali. ${ }^{20}$

Il confronto, la condivisione, lo scambio di conoscenze all'interno delle équipe è un propulsore che potrà generare un'evoluzione delle conoscenze nel singolo professionista. ${ }^{18}$

Circa l'evoluzione delle nursing clinical competence in area medica, ad oggi, l'unica pubblicazione che finalizza il focus sull'interezza di questo contesto clinico, risulta essere una pubblicazione del 2018, all'interno della quale, in linea con quanto pubblicato nel 2011 per la professione medica, ${ }^{21}$ vengono sistematizzate competenze specialistiche all'interno di tre distinti livelli di professionalità per ognuno dei seguenti processi di nursing: assistenza al paziente cronico, al paziente con scompenso cardiaco, con broncopneumopatia cronica e ostruttiva, cirrosi epatica, pancreatite acuta, diabete mellito, delirium, depressione, assistenza al paziente critico, al paziente con ipertensione arteriosa, trombosi venosa profonda, ischemia cerebrale, disfagia, squilibri idroelettrolitici, polmonite, sanguinamento gastro-intestinale, sepsi, assistenza al paziente con monitoraggio cardiaco, al paziente in ventilazione non invasiva, l'uso dell'ecografia operativa infermieristica, la somministrazione di terapia e chemioterapia, le lesioni da pressione, il dolore, gli accessi vascolari, il paziente fragile, il processo di dimissione difficile, l'assistenza al paziente in fine vita. ${ }^{22}$

La letteratura, pur affermando l'essenzialità da parte di ogni professionista nel sostenere l'aggiornamento continuo basato sulle migliori evidenze scientifiche, sottolinea come la quantità di nuove conoscenze prodotte quotidianamente renda irrealistico aspettarsi che ogni professionista riesca a stare costantemente aggiornato, ${ }^{23}$ ecco che quindi, per mantenere uno standard di aggiornamento ottimale è necessario trovare nuove modalità in grado di promuovere e divulgare pratiche basate sull'evidenza attraverso la progettazione di nuove strategie educative e di divulgazione delle conoscenze..$^{15}$

Nello studio di Cunningham è descritto come secondo il NHS England, il giusto mix di competenze del solo personale non garantirà la fornitura di cure di alta qualità ai pazienti e ciò dipende da una serie di altri fattori, come la leadership di un'organizzazione, la cultura di gestione, il team che lavora nel reparto, il livello di istruzione e la formazione disponibile per il personale e l'ambiente organizzativo. ${ }^{1}$

La competenza promuove la collaborazione tra l'infermiere e altri membri del team sanitario al fine di espandere e integrare nuove conoscenze e abilità nella propria pratica. ${ }^{5}$

È necessario, che le équipe siano incentrate su valori quali la condivisione e l'integrazione continua in un'ottica di collaborazione sinergica ed integrata per un continuo scambio di conoscenze e professionalità. ${ }^{18}$

La formazione e l'aggiornamento assumono un ruolo cardine per favorire lo sviluppo di competenze aggiornate che possano garantire la disseminazione di nuovo Know-how. ${ }^{18}$

Diverse ricerche e analisi della letteratura hanno rilevato una mancanza di attenta valutazione relativamente all'effetto che il contesto organizzativo ha sull'influenzare il processo decisionale relativo al mix di competenze. ${ }^{1}$

È quindi importante che il professionista sostenga l'apprendimento, e che l'organizzazione provveda a monitorare i livelli di competenza sviluppata/acquisita in coerenza con i risultati richiesti. ${ }^{20}$

L'aggiornamento continuo, il trasferimento di buone pratiche e nuove conoscenze sono i driver per migliorare l'assistenza, così come allo stesso tempo, per migliorare gli esiti delle persone assistite, sono fondamentali la volontà e la capacità di trasferire le conoscenze, le abilità e le attitudini. ${ }^{24}$

Laddove le organizzazioni supportano il luogo di lavoro come risorsa di apprendimento, i cambiamenti nella pratica professionale del singolo hanno un effetto diretto sul team e di questo possono beneficiarne sia l'organizzazione che il servizio. ${ }^{25}$

L'attività assistenziale quotidiana è risultata essere un'importante risorsa per l'apprendimento e il suo potenziale dovrebbe essere riconosciuto e sfruttato sistematicamente. ${ }^{3}$

Gli infermieri sono un gruppo professionale molto grande e riuscire ad identificare quali sono le strategie per promuovere l'adozione di pratiche evidence-based è fondamentale. ${ }^{26}$

Il continuo adattamento alle nuove necessità di salute e di conseguenza, il mantenimento di nuova conoscenza e lo sviluppo di competenze, necessita che l'intero sistema sia indirizzato verso livelli culturali che favoriscano opportunità di sviluppo e diffusione di competenze organizzate in termini di specificità di processi e risultati. ${ }^{20}$

\section{Bibliografia}

1. Cunningham J, O'Toole T, White M, Wells J. Conceptualizing skill mix in nursing and health care: An analysis. J Nurs Manag 2019;27:256-63.

2. Horsley T, O’Neill J, McGowan J, et al. Interventions to improve question formulation in professional practice and self-directed learning. Cochrane Database Syst Rev 2010 May 12 [cited 2019 Aug 24]; Available from: http://doi.wiley.com/10.1002/14651858.CD007335.pub 2

3. Pool I, Poell R, Berings M, Ten Cate O. Strategies for continuing professional development among younger, middle-aged, and older nurses: a biographical approach. Int J Nurs Stud 2015;52:939-50. 
4. Saiani L, Dimonte V, Palese A, et al. Il dibattito sullo sviluppo di competenze specialistiche/avanzate degli infermieri. Assist Inferm Ric 2016;35:116-21.

5. Axley L. Competency: A Concept Analysis. Nurs Forum 2008;43:214-22.

6. White KR, Pillay R, Huang X. Nurse leaders and the innovation competence gap. Nurs Outlook 2016.

7. Weng YH, Kuo KN, Yang CY, et al. Increasing utilization of Internet-based resources following efforts to promote evidence-based medicine: A national study in Taiwan. BMC Med Inform Decis Mak 2013;13(1).

8. Rodriguez C, Victor C, Leonardi N, et al. Barriers to participation in an online nursing journal club at a community teaching hospital. J Contin Educ Nurs 2016;47: 536-42.

9. Doran D, Haynes BR, Estabrooks CA, et al. The role of organizational context and individual nurse characteristics in explaining variation in use of information technologies in evidence based practice. Implement Sci 2012;7(1).

10. Oermann MH, Floyd JA, Galvin EA, Roop JC. Brief reports for disseminating systematic reviews to nurses. Clin Nurse Special 2006;20:233-8.

11. Straus S, Haynes R. Managing evidence-based knowledge: the need for reliable, relevant and readable resources. CMAJ 2009;180:9.

12. Loveday HP, Wilson JA, Pratt RJ, et al. Epic3: National evidence-based guidelines for preventing healthcare-associated infections in NHS Hospitals in England. J Hosp Infect 2014;86(S1).

13. Bathish M, Wilson C, Potempa K. Deliberate practice and nurse competence. Appl Nurs Res 2018;40:106-9.

14. Levati W, Saraò M. Il modello delle competenze. Milano: FrancoAngeli; 2003. 127 p.

15. Conway A, Dowling M, Binchy A, et al. Implementing an initiative to promote evidence-informed practice: part 1 - a description of the Evidence Rounds programme. BMC Med Educ 2019;6;19:74.

16. Flodgren G, O’Brien MA, Parmelli E, Grimshaw JM. Local opinion leaders: Effects on professional practice and healthcare outcomes. Cochrane Datab Syst Rev 2019;2019.

17. Acquaro J, Tousijn W. Gestire la cronicità: Potere, Responsabilità e Decision Making. Prof Inferm [Internet]. 2019;72:3.

18. Lega F, Mauri M, Prenestini A. L'ospedale tra presente e futuro. Analisi, diagnosi e linee di cambiamento per il sistema ospedaliero italiano. 1st ed. Milano: EGEA; 2010. pp 303-304.

19. Bressan V, Stevanin S. La competenza culturale: modelli, standard e strumenti per l'infermiere italiano. L'infermiere [Internet] 2014;2. Available from: www.ipasvi.it

20. Borgioli M, Chiappi S, Bini B, Rasero L. Un sistema per valutare le competenze nell'infermieristica: la definizione degli strumenti operativi. Prof Inferm [Internet] 2014;63:4. Available from: http://www.profinf.net.bvs2. clas.cineca.it/pro3/index.php/IN/article/view/62/47

21. Nardi R, Mathieu G, Berti F, et al. Modelli di valutazione della clinical competence del medico specialista internista ospedaliero. Ital J Med 2011;5:33-45.

22. Bertoncini F, Gatta C, Pentella G. Nursing clinical competence in area medica. Ital J Med 2018 Oct 24;1-83.

23. Registered Nurses' Association of Ontario. Toolkit : Implementation of Best Practice Guidelines. Best Pract Guidel [Internet] 2012;(September):1-154. Available from: http://rnao.ca/sites/rnao-ca/files/RNAO_ToolKit_2012 rev4_FA.pdf

24. Wellings C, Gendek M, Gallagher S. Evaluating Continuing Nursing Education. A Qualitative Study of Intention to Change Practice and Perceived Barriers to Knowledge Translation. J Nurses Prof Dev 2017;33:6.

25. Manley K, Martin A, Jackson C, Wright T. A realist synthesis of effective continuing professional development (CPD): A case study of healthcare practitioners CPD. Nurse Educ Today 2018.

26. Wu Y, Brettle A, Zhou C, et al. Do educational interventions aimed at nurses to support the implementation of evidence-based practice improve patient outcomes? A systematic review. Nurse Educ Today 2018;50:109-14. 


\title{
Gli esiti sensibili all'assistenza infermieristica in Medicina Interna
}

\author{
Letizia Tesei, ${ }^{1}$ Claudia Gatta ${ }^{2}$ \\ ${ }^{1}$ Gruppo Ricerca Associazione ANiMO, Area Vasta 2 ASUR Marche, Ancona; ${ }^{2}$ Direttivo ANiMO, Azienda Sanitaria Locale, \\ Biella, Italia
}

\section{Introduzione}

L'ipotesi che l'assistenza infermieristica sia fondamentale per ottenere risultati di qualità relativamente alla salute delle persone, delle famiglie e della comunità è alla base della disciplina infermieristica. A tale proposito, in letteratura si trovano diverse definizioni di Nursing Sensitive Outcomes o Nursing Outcomes (esiti infermieristici), tutte riconducibili al contributo dell'assistenza infermieristica nel percorso di cura del paziente. Pur non esistendo ancora una definizione universale, per esiti infermieristici si intende una condizione, un comportamento o una percezione misurabile del paziente o della sua famiglia, concettualizzata come variabile e largamente influenzata $d a$ $o$ sensibile alle cure infermieristiche. Gli esiti sensibili all'infermieristica possono anche essere definiti come i cambiamenti misurabili nella condizione del paziente, attribuiti alla cura infermieristica ricevuta. Ad oggi gli esiti sensibili alle cure infermieristiche possono essere classificati a seconda dell'ambito al quale si riferiscono in: clinici e di sicurezza, organizzativi e relativi all'ambiente lavorativo, soggettivi dei pazienti e degli operatori. Nelle Medicine interne gli esiti sensibili alle cure infermieristiche rappresentano la conseguenza o gli effetti degli interventi erogati dagli infermieri e si manifestano in prevenzione dei rischi correlati all'assistenza, con cambiamenti nello stato di salute, nel comportamento o nella percezione delle persone assistite.

A partire dalla fine degli anni ' 90 sono stati istituiti a livello internazionale osservatori sugli esiti correlati

Corrispondente: Letizia Tesei, Gruppo Ricerca Associazione ANiMO, Area Vasta 2 ASUR Marche, Ancona, Italia. E-mail: letizia.tesei@hotmail.it

Articolo pubblicato secondo la Creative Commons Attribution NonCommercial 4.0 License (CC BY-NC 4.0).

${ }^{\circ}$ Copyright: the Author(s), 2020

Licensee PAGEPress, Italy

QUADERNI - Italian Journal of Medicine 2020; 8(3):78-84 alle cure infermieristiche attraverso set di indicatori prestabiliti al fine di misurare la performance assistenziale. ${ }^{1}$ Quando infatti l'assistenza infermieristica non è ottimale, i pazienti sperimentano negativamente l'assistenza sanitaria ricevuta. ${ }^{2}$ La mancata garanzia della qualità dell'assistenza infermieristica, non porta solo insoddisfazione e angoscia, ma anche a fallimenti in termini di sicurezza dei pazienti. Studi a livello internazionale hanno messo in evidenza le conseguenze dannose della scarsa assistenza infermieristica. ${ }^{3}$ In particolare, l'assistenza mancata o incompleta è stata identificata quale meccanismo chiave che spiega l'associazione ampiamente dimostrata tra i livelli di personale infermieristico e gli esiti del paziente, inclusa la mortalità. ${ }^{4,5} \mathrm{~A}$ tale proposito, la ricerca negli ultimi 20 anni, ha dimostrato l'impatto del numero e della composizione del personale sugli esiti sul paziente ed è ormai noto che nei contesti in carenza di personale gli esiti sui pazienti peggiorano. Dove esiste un rapporto infermieri pazienti più elevato diminuiscono le situazioni in cui gli infermieri non riescono a riconoscere le criticità del paziente, le cadute, la durata della degenza e le riammissioni. Di contro, dove c'è meno personale, aumentano gli errori di terapia e le cure omesse. ${ }^{6}$ È stata particolarmente studiata l'associazione tra rapporto infermieri/pazienti e aumento della mortalità. ${ }^{7}$ Di recente pubblicazione sono i risultati dello studio di AIKEN et al. Nell'ambito del Progetto Registered Nurse Forecast - RN4CAST, è stata correlata la mortalità ospedaliera a 30 giorni dall'ammissione alle variabili dello staff infermieristico: carico di lavoro e formazione di base. Fra i numerosi esiti sensibili alle cure infermieristiche studiati (es., lesioni da pressione,cadute, contenzione, ecc.) ci sono anche le infezioni correlate all'assistenza, un esito tra i più studiati unitamente alla mortalità, nonché quelli per i quali sono state dimostrate correlazioni statisticamente significative con la quantità di assistenza infermieristica erogata. ${ }^{8}$

\section{Criteri e strumenti di ricerca}

È stata condotta una revisione della letteratura al fine di sintetizzare i principali studi (qualitativi e quantitativi) con l'obiettivo di consentire una compren- 
sione esaustiva del fenomeno indagato. Non è stata prevista tuttavia un'analisi della qualità degli studi considerati. La ricerca bibliografica è stata condotta consultando attraverso la piattaforma ESBCO le banche dati Medline e Cinahl, il motore di ricerca Google Scholar, la ricerca manuale nelle riviste. Per la ricerca sono stati utilizzati i termini: Nursing Sensitive Outcomes, Nursing Outcomes.

\section{Risultati}

\section{Esiti sensibili alle cure infermieristiche}

Rispetto agli esiti correlati alle cure infermieristiche, anche se il dibattito è recente, in passato, alcune teoriche dell'infermieristica avevano già contribuito ad individuarli. Florence Nightingale ad esempio, fu la prima ad ipotizzare anche per l'infermieristica misure di esito tipiche della medicina (mortalità e morbilità), mentre Virginia Henderson dichiarò l'indipendenza del paziente nelle attività di vita quotidiane (ADL) quale esito più importante per valutare l'assistenza infermieristica. ${ }^{9}$ Ma è solo a partire dalla fine degli anni ' 90 che sono stati istituiti a livello internazionale osservatori sugli esiti correlati alle cure infermieristiche con l'obiettivo di monitorare gli esiti dell'assistenza infermieristica sul paziente e quindi misurare la performance assistenziale. Allo scopo di superare la carenza di strumenti validati, l'associazione delle Infermiere dello Stato di Alberta (Canada), ha realizzato nel 1993, una prima revisione delle pubblicazioni disponibili. Successivamente, nel 1997, l'università dell'Iowa (USA) ha sviluppato il Nursing Outcomes Classification (NOC) che ha guidato il dibattito internazionale per anni sui risultati clinici degli infermieri. ${ }^{10}$ Diane Doran e Peter Griffiths sono tra i principali autori che hanno cercato di dare una visione globale della letteratura sugli outcome. Doran ad esempio definisce i nursing sensitive outcome come la conseguenza o gli effetti degli interventi erogati dagli infermieri che si manifestano con cambiamenti nello stato di salute, nel comportamento o nella percezione del paziente e/o con la risoluzione del problema attuale per il quale l'assistenza infermieristica è stata prestata. ${ }^{1}$ Griffiths invece, descrive i nursing sensitive outcomes come aspetti dell'esperienza, del comportamento e dello stato di salute del paziente che sono determinati in tutto o in parte dall'assistenza infermieristica ricevuta, con variazioni che dipendono dalla qualità e dalla quantità dell'assistenza stessa. ${ }^{11}$ Inoltre, per definire gli esiti dell'assistenza, si è cercato di inquadrare gli stessi in modelli concettuali di riferimento, correlandoli con le caratteristiche della assistita. Fra i modelli sviluppati per l'applicazione in ambiente ospedaliero, un sistema ha sistematizzato gli esiti assistenziali in categorie come l'educazione dell'assistito e della sua famiglia, le capacità di autocura, la gestione dei sintomi, il livello di sicurezza dell'assistito ed il suo livello di soddisfazioni; un altro prende in considerazione le condizioni fisiologiche e psicologiche, la conoscenza della propria salute ed il livello di soddisfazione; un altro ancora ha considerato lo stato funzionale, lo stato mentale, il livello di stress e di soddisfazione dell'assistito, i costi dell'assistenza. ${ }^{1}$ Ad oggi, gli esiti sensibili alle cure infermieristiche possono essere classificati a seconda dell'ambito al quale si riferiscono in clinici e di sicurezza, organizzativi e relativi all'ambiente lavorativo, soggettivi dei pazienti e degli operatori. ${ }^{1}$

\section{Esiti sensibili alle cure infermieristiche in Medicina Interna}

\section{Riduzione abilità ADL - indipendenza - qualità di vita}

Il rischio di declino funzionale post-ricovero è stato ampiamente studiato, ${ }^{12}$ ma poche ricerche hanno esplorato l'associazione tra processi di cura e outcome funzionali post-ricovero, nonostante esistano evidenze a sostegno dell'associazione tra questi ultimi e la ridotta mobilità, l'isolamento sensoriale, l'ambiente estraneo ed ostile e il diminuito apporto nutrizionale durante il periodo di degenza tutti fattori che concorrono a un peggioramento della qualità di vita. Diversi studi hanno dimostrato che i pazienti, pur in grado di deambulare al momento del ricovero, hanno trascorso a letto $1^{\prime} 83,3 \%$ della giornata, seduti per il $12,9 \%$, e in piedi o a camminare per il 3,8\% del tempo. Questo dato è particolarmente importante per l'area medica in quanto gli anziani rappresentano oltre il $70 \%$ dei pazienti degenti. Ma, fattori diversi dalla dipendenza nella mobilità di base rappresentano le cause principali per cui i pazienti rimangono inattivi durante il ricovero. In particolare, il vissuto dei professionisti sanitari circa la mobilizzazione dei pazienti, soprattutto quando questi necessitano di assistenza per alzarsi dal letto, sollevarsi da una sedia o camminare, chiedendo una maggiore attenzione da parte del personale di reparto, può rappresentare un fattore incidente sul livello di mobilità del paziente ricoverato. Lo studio di So e Pierluissi, ha dimostrato per esempio, come i pazienti anziani degenti in ospedale hanno una percezione positiva rispetto all'esercizio fisico e che i professionisti sanitari hanno una forte influenza in tale attività durante la degenza. La maggior parte dei partecipanti allo studio, pur ritenendo l'attività fisica importante, non pensava di doverla praticare durante il ricovero. Gli stessi dichiarano che i medici e gli infermieri li hanno poco stimolati ad esercitarsi. Poiché il livello di mobilità è legato agli outcome funzionali dei pazienti, è importante sviluppare e valutare strategie per 
una mobilizzazione efficace e precoce dei pazienti anziani ricoverati nelle medicine interne. Gli infermieri in particolare, dovrebbero concentrare i loro sforzi per elaborare protocolli di intervento atti a promuovere la mobilità e prevenire il declino funzionale

\section{Deterioramento clinico improvviso}

Le ricerche dimostrano che 1' $80 \%$ dei pazienti che sono ricoverati nei setting di area medica non sono monitorati tramite dispositivi elettronici, il $20 \%$ dei pazienti monitorati, per le condizioni cardiache e respiratorie, non hanno dispositivi di monitoraggio continuo, inoltre, studi dimostrano che, quando il numero dei pazienti assegnata ciascun infermiere supera le 4 unità, la mortalità, le complicanze e i casi di morte improvvisa aumentano. ${ }^{13}$ La vigilanza è elemento essenziale della cura infermieristica e come tale, definisce il ruolo chiave degli infermieri all'interno del sistema sanitario, duemila pazienti muoiono ogni anno in ospedale, a causa del ridotto livello di sicurezza presente. Nei reparti medici, le ragioni possono includere un insufficiente numero di personale, e un insufficiente o inefficace sistema di identificazione del deterioramento clinico. Inoltre, studi evidenziano come ogni decisione clinica degli infermieri è influenzata dal livello di stanchezza e dall'essere soli in turno o in pochi. Dati significativi suggeriscono infine che la notte insieme alla mattina presto rappresentano i momenti in cui il paziente è a maggior rischio di morte improvvisa. A tal proposito un' adeguata sorveglianza dovrebbe essere garantita, utilizzando particolari protocolli e nuove tecnologie in grado di allertare lo staff infermieristico relativamente al deterioramento clinico dei pazienti.

\section{Delirium}

Il delirium ${ }^{14}$ nell'adulto, è una condizione severa associata ad esiti negativi per il paziente la quale può essere prevenuta e i sintomi trattati se riconosciuti e affrontati con urgenza. Circa il $20-30 \%$ dei pazienti ricoverati nei reparti medici manifestano delirium. Il delirium e scarsamente riconosciuto e poco registrato nella documentazione clinica ma il suo riconoscimento e tempestivo trattamento può impedire il manifestarsi di importanti complicanze come: aumentato rischio cadute e lesioni da decubito (cinque volte maggiore); prolungamento della degenza (da 9 a 21 giorni).

Il trattamento della sintomatologia del delirium oltre che di una componente farmacologica necessita di una componente non farmacologica della quale gli infermieri sono responsabili. In particolare l'assistenza infermieristica anche attraverso il coinvolgimento della famiglia e caregiver, mira al recupero dell'orientamento del paziente e al miglioramento delle capacita funzionali, della mobilità, alla preven- zione delle cadute, lesioni da pressione, infezioni e declino funzionale.

\section{Lesioni da pressione}

Il rischio di sviluppare ulcere da pressione ${ }^{15}$ riguarda qualunque paziente allettato per brevi, medi o lunghi periodi. La letteratura mostra che i dati maggiori sono in riferimento a pazienti anziani fragili che sviluppano ulcere da pressioni a causa di immobilizzazione e allettamento, le lesioni da pressione (LdP) sono un fenomeno presente, e sempre più in aumento per due fondamentali cause: l'aumento dell'età media dei pazienti e, soprattutto in Italia, la carenza, sempre più grave di personale all'interno dei reparti, che comporta un'impossibilita di mettere in atto tutte le manovre di prevenzione necessarie per diminuire il rischio di comparsa delle lesioni e non aggravare lesioni già presenti. in Italia i dati relativi ad uno studio nazionale di prevalenza che ha valutato 13.081 pazienti ricoverati in 24 strutture ospedaliere, ha documentato un tasso di prevalenza pari al $10,97 \%$, che non si associava però, ad un'adeguata risposta in termini di applicazione di misure preventive, che infatti venivano adottate soltanto nel $9,4 \%$ dei casi.

\section{Conoscenze per prevenzione/gestione complicanze collegate alla propria patologia}

L'educazione all'autogestione della propria patologia è un processo di facilitazione delle conoscenze, abilità e comportamenti fondamentale nella sua cura. ${ }^{16}$ Essa ha come obiettivo quello di fare sviluppare la capacità di prendere decisioni da parte della persona in genere con malattia cronico/degenerative che, così, farà parte a tutti gli effetti del team di cura con cui condivide obiettivi di miglioramento dei risultati clinici, dello stato di salute e della qualità di vita. Anche se le revisioni sistematiche sulla terapia educativa nei pazienti cronici rilevano l'eterogeneità nei metodi e nella modalità di riportare i risultati degli studi citati, molti studi hanno riscontrato che l'educazione all'autogestione della malattia cronica si associa a: miglioramento della conoscenza della malattia, miglioramento nelle modalità di autocura, miglioramento negli esiti. La presenza di un'attività infermieristica nel coordinamento degli interventi educativi aumenta l'efficacia degli stessi a breve termine. Nonostante i risultati incoraggianti presenti in letteratura, l'applicazione degli interventi educativi nella realtà clinica presenta ancora grosse difficoltà. Il tempo dedicato dai diversi operatori sanitari all'attività educativa copre una parte minima dell'orario settimanale, l'educazione viene svolta in molti casi in maniera non strutturata, solo poco più della metà dei centri che fanno educazione terapeutica attua viene eseguita la valutazione e la registrazione dell'attività educativa. Poiché gli infermieri giocano 
un ruolo importante e hanno una precisa responsabilità nell'assistenza delle persone con malattie croniche, hanno il dovere di iniziare il prima possibile il selfmanagement mirato sulla patologia.

\section{Aderenza terapeutica, competenza sulla somministrazione dei farmaci}

L'aderenza del paziente alle prescrizioni terapeutiche, rappresenta uno dei problemi più importanti della pratica clinica, dal momento che il successo di qualsiasi intervento terapeutico dipende dalla effettiva adesione del paziente alla prescrizione. ${ }^{17} \mathrm{La}$ non aderenza ha infatti come dirette conseguenze, la distorsione dell'efficacia del trattamento e l'emergere di problematiche connesse ad un incremento dei costi legato all'inefficace gestione della patologia. L'Organizzazione Mondiale della Sanità, afferma che l'aderenza a lungo termine alla terapia farmacologica per le malattie croniche nei paesi sviluppati, corrisponde ad una media del 50\%. In generale, la non aderenza terapeutica si traduce in un peggioramento dei sintomi e frequenti ri-ospedalizzazioni ed è inoltre fonte di preoccupazione per diversi motivi. Non assumere o assumere meno farmaci di quelli prescritti può portare al non raggiungimento dell'outcome di trattamento. Molte persone inoltre, non riferiscono ai propri curanti quando non stanno assumendo la terapia. Questo può determinare una valutazione sbagliata delle condizioni del paziente. Comprendere ed analizzare il complesso concetto di aderenza terapeutica può aiutare quindi i professionisti sanitari e quindi gli infermieri a migliorare l'assistenza alla persona. Capire le cause di non aderenza è essenziale per il raggiungimento degli esiti clinici e comportamentali desiderati. Poiché nei nostri contesti di lavoro, i pazienti sono spesso fragili, presentano problemi di tipo cognitivo comportamentale, e quindi non sono in grado di gestire autonomamente le terapie farmacologiche prescritte, è importante valutare la capacità del familiare o del caregiver di gestire i farmaci prescritti ed eventualmente attuare su questi interventi mirati. Infatti, affinché i programmi di assistenza per le condizioni croniche si rivelino efficaci, è necessario che il paziente, la famiglia e la comunità che lo/la sostengono, giochino contemporaneamente un ruolo attivo. Il sostegno sociale, sia formale che informale, che il paziente riceve da altri membri della sua comunità, è stato registrato come un importante fattore capace di influenzare gli outcome positivi sulla salute e sul comportamento.

\section{Cadute}

Uno degli aspetti ancora molto dibattuto in sanità a livello mondiale, proprio per la sua intrinseca complessità multifattoriale in termini di prevedibilità, gestione ed implicazioni, e il problema delle cadute negli anziani. ${ }^{18}$ Sebbene l'incidenza sia maggiore nella popolazione anziana, sono da ritenere a rischio tutte le persone che accedono alle strutture sanitarie poiché in fase acuta, o perché caratterizzate da particolari patologie (ad esempio persone affette da cancro in fase terminale) o da situazioni fisiologiche (ad esempio donne in gravidanza con una postura sbilanciata o episodi lipotimici). Secondo l'OMS il 30\% delle persone sopra i 65 anni cade ogni anno e per le persone sopra ai 75 anni i tassi sono più elevati. Ed ancora il $78 \%$ delle cadute che si verificano in ospedale e rappresentato da cadute prevedibili. La valutazione del rischio deve essere effettuata mediante strumenti standardizzati e attraverso la valutazione clinica del professionista, elemento irrinunciabile nella definizione del rischio, in quanto nessuno strumento di valutazione, da solo, è in grado di identificare tutte le persone a rischio o tutti i fattori di rischio. È inoltre dimostrato che gli interventi multifattoriali mirati sono in grado di ritardare e ridurre le cadute dei pazienti ospiti nelle residenze assistenziali. Essi comprendono: esercizio fisico; revisione delle terapie farmacologiche; modifiche ambientali; ausili per la deambulazione; uso di calze antiscivolo negli anziani; formazione del personale; educazione sanitaria; problem solving dopo la caduta.

\section{Malnutrizione}

La malnutrizione ${ }^{19}$ è una condizione caratterizzata da carenza di sostanze nutritive, che è causa di esiti negativi misurabili. In particolare, è dimostrato come in ospedale, la malnutrizione influisce sull'aumento delle infezioni delle ferite, delle ulcere da pressione, sui tempi di degenza, sui re-ricoveri e sulla mortalità. Il rischio di malnutrizione aumenta con l'età e con i livelli di cura; nonostante i significativi progressi della medicina, la malnutrizione rimane un problema di salute pubblica con alta prevalenza nei paesi in via di sviluppo. Sebbene i dati siano diversi da uno studio all'altro, evidenze disponibili mostrano che un intervento nutrizionale precoce può ridurre i tassi di complicanze e il costo delle cure. Al fine di intervenire tempestivamente, è quindi importante identificare, la malnutrizione $\mathrm{o}$ il rischio già al momento dell'ammissione in ospedale, in modo precoce e sistematico. Le evidenze scientifiche riportano come il $20-40 \%$ dei pazienti presentino, alla loro ammissione in ospedale, un quadro di malnutrizione, situazione destinata a peggiorare durante la degenza. Infatti, è stato confermato che $1170 \%$ dei pazienti presenta un peggioramento del proprio stato nutrizionale durante i primi 10 giorni di ricovero. La popolazione anziana rappresenta un gruppo vulnerabile ad elevato rischio di malnutrizione in quanto l'invecchiamento è associato a deterioramento fisiologico, a menomazione fisica e psico-sociale e spesso a difficoltà economiche, fattori che 


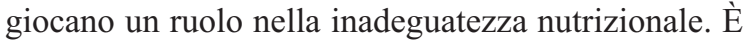
importante quindi, che gli infermieri e tutto il personale sanitario valutino lo stato nutrizionale di ogni paziente al fine di identificare la malnutrizione e cercare di gestire i problemi nutrizionali, utilizzando strumenti di screening pratici, rapidi e affidabili. La letteratura riporta diversi metodi validati che possono facilitare l'identificazione dei fattori di rischio di malnutrizione. Per migliorare l'assistenza nutrizionale è necessario però che l'organizzazione all'interno delle unità operative preveda screening e programmi assistenziali tali da soddisfare le esigenze dei singoli degenti. Il miglioramento della pratica clinica relativa alla gestione del problema della malnutrizione, offre una straordinaria opportunità per ottimizzare la qualità complessiva della cura del paziente, migliorare i risultati clinici e ridurre i costi.

\section{Le infezioni correlate all'assistenza}

Secondo uno studio condotto tra il 2011 ed il 2012 dallo European Centre for Disease Prevention and Control, in oltre 30 Pesi europei, la prevalenza media di pazienti ricoverati con almeno una infezione correlata all'assistenza $(\mathrm{ICA})^{20}$ era del $5,7 \%$ (Italia 6.1 IC95\% 4.8-9.6). Le ICA sono, unitamente alla mortalità, tra gli esiti di cura più studiati, nonché quelli per i quali sono state dimostrate correlazioni statisticamente significative con l'assistenza infermieristica. I risultati della revisione della letteratura mettono in luce l'associazione tra le variabili di staffing e gli esiti di tipo infettivologico. La variabile di staffing maggiormente studiata è stata il numero di ore di assistenza infermieristica per ogni paziente. Anche il rapporto numerico infermiere/paziente, il clima organizzativo e i livelli di formazione infermieristica avanzata, sono stati associati alle infezioni.

\section{La soddisfazione delle cure}

Gli indicatori di soddisfazione delle cure (caring $)^{21}$ identificati dai pazienti sono coerenti con quelli individuati in un'ampia ed esaustiva analisi concettuale del concetto di cura: il prestare attenzione inteso come capacità di far posto all'altro nella mente, i pazienti percepiscono di ricevere caring quando si sentono protetti e controllati, non si sentono soli, si rendono conto di essere nei pensieri degli infermieri perché tornano ad osservarli, a misurare dei parametri, a sorvegliare l'evoluzione delle loro condizioni senza doverli chiamare.

Essere responsivi significa saper rispondere alle necessità dell'altro con sollecitudine, con prontezza, quindi dare una risposta tempestiva, sollecita, ma anche quando mantenere le promesse.

La disponibilità cognitiva e emotiva, che si esprime con una relazione densa, con la presenza, viene apprezzata e percepita dai pazienti quando l'in- fermiere si interessa alla loro storia, al loro sentire (sentirsi riconosciuti e rispettati nella propria individualità), quando condivide anche parti di sé per stare ancora più vicino nella relazione.

La competenza tecnica è la condizione essenziale per un buon esercizio della cura; non viene menzionata ma i pazienti intervistati assegnano molto valore alla componente informativa. Informazioni puntuali e appropriate vengono apprezzate non solo per il loro contenuto ma soprattutto perché transitano interesse, attenzione, preoccupazione e quindi come un momento di intenso caring.

Gli attuali modelli assistenziali, ad esempio il Primary Nursing, il Case Management dove vengono assegnate agli infermieri precise responsabilità verso un gruppo di pazienti sono sicuramente una condizione facilitante.

\section{La contenzione}

Il Royal College of Nursing definisce la contenzione come una restrizione intenzionale dei movimenti o del comportamento volontario del soggetto.

Se la contenzione può essere considerata quale Atto sanitario assistenziale che utilizza, con o senza il consenso della persona elo dei famigliari, mezzi fisici o meccanici applicati al corpo o allo spazio circostante la persona per limitarne i movimenti, il concetto di atto sanitario assistenziale, richiama quelli di prevenzione e di cura. L'obiettivo che si prefigge la contenzione deve essere pertanto, esclusivamente l'aiuto assistenziale, ovvero la salvaguardia dell'incolumità psicofisica del paziente. Se è vero che la contenzione in alcune circostanze è necessaria per la sicurezza del soggetto e di chi lo assiste è altrettanto vero che va utilizzata con coscienza e solo in caso di effettiva necessità.

La contenzione infatti, non solo rappresenta una limitazione della libertà della persona, ma può avere ripercussioni sul piano psicologico del paziente e dei familiari e causare una serie di conseguenze fisiche al paziente contenuto. A tale proposito, a tutela del diritto alla salute e della libertà individuale intervengono sia il Codice Penale che il Codice Deontologico delle Professioni Infermieristiche; quest'ultimo, al Capo VI Art. 35, cita testualmente: L'Infermiere riconosce che la contenzione non è atto terapeutico. Essa ha esclusivamente carattere cautelare di natura eccezionale e temporanea; può essere attuata tura dall'equipe o, in caso di urgenza indifferibile, anche dal solo Infermiere se ricorrono i presupposti dello stato di necessità, per tutelare la sicurezza della persona assistita, delle altre persone e degli operatori. La contenzione deve comunque essere motivata e annotata nella documentazione clinico assistenziale, deve essere temporanea e monitorata nel corso del tempo per verificare se permangono le condizioni che ne hanno 
giustificato l'attuazione e se ha inciso negativamente sulle condizioni di salute della persona assistita. ${ }^{22}$

Nonostante la scarsità degli studi in merito, più del profilo comportamentale o psicologico dei pazienti e della quantità di risorse a disposizione, pare rivestano un ruolo decisivo la cultura, l'organizzazione dei servizi e l'attitudine degli operatori. La contenzione fisica è stata recentemente inclusa nel concetto più generale di maltrattamento degli anziani assieme alla contenzione farmacologica e ambientale: prevenirla ed eliminarla significa ridurre il maltrattamento perché accanto alla contenzione sono spesso attuate altre pratiche restrittive (es., applicazione di pannoloni assorbenti in assenza di appropriata indicazione) ${ }^{23}$

Zanetti et al., hanno elaborato degli studi di monitoraggio della contenzione fisica anche per sensibilizzare tutti gli operatori, e anche i famigliari, a questa problematica cercando di equilibrare il bisogno di sicurezza/protezione con il rispetto della dignità della persona. Inoltre stanno stimolando la riflessione critica sulle pratiche assistenziali a partire dall'analisi del quotidiano e per dare voce ad un fenomeno sul quale non c'è ancora molta chiarezza e se ne parla ancora troppo poco. ${ }^{24}$

$\mathrm{E}$, inoltre molto importante chiarire il concetto di falsa contenzione, la contenzione è la situazione in cui si applicano ad una persona, non in grado di comprenderne le finalità/utilità, in assenza di una sua richiesta o consenso, mezzi che impediscono la libertà di movimento e che non possono essere rimossi senza l'aiuto di altri. Le false contenzioni sono invece le situazioni in cui vengono applicati dispositivi per favorire il mantenimento/recupero delle ADL ed il comfort ad una persona in grado di comprenderne le finalità/utilità o per lo meno non manifesta contrarietà al loro utilizzo. La mancanza di chiarezza tra contenzione quale cattiva pratica (funzionale al mantenimento di organizzazioni che non curano) e contenzione quale garanzia della postura o per aumentare l'autonomia, rende difficile non solo la raccolta dati e il loro proficuo utilizzo ma sollecita profondi dilemmi professionali. Per questo sarebbe necessario chiarire quanto prima, a livello istituzionale, organizzativo e professionale questa ambivalenza fonte di tante incomprensioni.

Inoltre, focalizzare l'attenzione sulle diverse tipologie di contenzioni i cui significati ed utilità non possano essere compresi e condivisi da chi le subisce, apre la discussione anche sulla dimensione del consenso, su quando questo vada ricercato e acquisito nelle situazioni in cui è facile prevedere che la persona non sarà più in grado di esprimerlo (programmazione anticipata delle cure) e riporta la discussione sul tema dell'autonomia sostenibile. ${ }^{23}$

Proprio a questo scopo Animo ha indicato, nel progetto Choosing Wisely fare di più non vuol dire fare meglio la contenzione come pratica a rischio di inappropriatezza, e ha contribuito a costruire una scheda informativa sul problema con Altro Consumo.

\section{Conclusioni}

L'attenzione della ricerca ai risultati correlati all'assistenza rappresenta la chiave per monitorare e migliorare la qualità delle cure e richiede un cambiamento culturale della pratica clinica. Sebbene la qualità e la sicurezza delle cure sia riconosciuta come una sfida interprofessionale, nell' ambito di questa sfida è stato dimostrato che alcune tipologie di outcome sono sensibili e dipendono dall'assistenza infermieristica. ${ }^{1}$

Gli infermieri hanno pertanto la responsabilità di valutare l'effetto della pratica infermieristica sugli esiti dei pazienti considerando aspetti quali la promozione della salute, la prevenzione dei rischi e delle malattie e la riduzione della sofferenza. ${ }^{25}$ Se nel tempo, le iniziative di ricerca sulla valutazione della qualità e sui risultati sono state ostacolate dalla mancanza di dati pertinenti disponibili, è fondamentale oggi investire risorse finalizzate alla raccolta dei dati che potranno sempre più fornire misure affidabili e valide dei processi di assistenza infermieristica e dei risultati ottenuti, a supporto del contributo dell'infermiere alla cura del paziente. ${ }^{18} \mathrm{Il}$ monitoraggio degli esiti sensibili alle cure infermieristiche, anche in area medica, dovrebbe costituire parte integrante della normale pratica clinica per la valutazione degli interventi di assistenza, come avviene nei sistemi sanitari avanzati.

La misurazione degli esiti sensibili alle cure infermieristiche, consentirà di migliorare la sicurezza delle cure, la responsabilità del personale sugli esiti, la cultura della valutazione e di produrre informazioni utili al management infermieristico per prendere decisioni sull'organizzazione delle cure ed intraprendere un dialogo costruttivo sulle pratiche sicure.

\section{Bibliografia}

1. Doran D. Nursing Outcomes - Gli esiti sensibili alle cure infermieristiche. London: McGraw-Hill; 2013.

2. Rathert C, Wyrwich MD, Boren SA. Patient-centered care and outcomes: A systematic review of the literature. Med Care Res Rev 2013;70;351-79.

3. Griffiths P, Recio-Saucedo A, Dall'Ora C, et al. The association between nurse staffing and omissions innursing care: A systematic review. Adv Nurs 2018;74:1474-87.

4. Aiken LH, Sloane DM, Bruyneel L, et al. Nurse staffing and education and hospital mortality in nine European countries: A retrospective observational study. Lancet 2014;383:1824-30.

5. Ball JE, Murrells T, Rafferty AM, et al. Care left undone during nursing shifts: Associations with workload and 
perceived quality of care. BMJ Qual Saf 2014;23:116-25.

6. Mongardi M, Fortuna D, Sciolino L. Gli esiti sensibili alle cure infermieristiche: risultati di uno studio multicentrico. Assist Inferm Ric 2019;38:117-37.

7. McHugh MD, Ma C. Hospital Nursing and 30-Day Readmissions among Medicare Patients with Heart Failure, Acute Myocardial Infarction, and Pneumonia. Med Care 2013;51:52-9.

8. Aiken LH, Cerón C, Simonetti M, et al. Hospital nurse staffing and patient outcomes. Rev Méd Clínica Las Condes 2018;29:322-7.

9. Palese A, Beltrame ER, Bin A, et al. E siti sensibili alle cure infermieristiche: analisi critica della letteratura. Assist Inferm Ric 2008;27:33-42.

10. Mongardi M, Bassi E, De Palma R, et al. Osservatorio regionale sui nursing sensitive Outcomes. Servizio Presidi Ospedalieri - DGSPS, Regione Emilia Romagna; 2014.

11. Griffiths P, Jones S, Maben MT. State of the art metrics for nursing : a rapid appraisal. London: National Nursing Research Unit at King's College; 2008.

12. Bertoncini F, Di Berardino S, Lo Burgio I, et al. La ricerca finalizzata in Area Medica: approfondimenti assistenziali dell'Associazione A.N.Í.M.O. Quaderni Ital J Med 2019;7:40-2.

13. Bertoncini F, Di Berardino S, Lo Burgio I, et al. La ricerca finalizzata in Area Medica: approfondimenti assistenziali dell'Associazione A.N.Í.M.O. Quaderni Ital J Med 2019;7:45-46.

14. Bertoncini F, Gatta C, Pentella G. Nursing clinical competence in area medica. Quaderni Ital J Med 2018;6:13-4.

15. Bertoncini F, Gatta C, Pentella G. Nursing clinical competence in area medica. Quaderni Ital J Med 2018;6:41-2.

16. Bertoncini F, Di Berardino S, Lo Burgio I, et al. La ricerca finalizzata in Area Medica: approfondimenti assistenziali dell'Associazione A.N.Í.M.O. Quaderni Ital J
Med 2019;7:47-8.

17. Bertoncini F, Di Berardino S, Lo Burgio I, et al. La ricerca finalizzata in Area Medica: approfondimenti assistenziali dell'Associazione A.N.Í.M.O. Quaderni Ital J Med 2019;7:33-4.

18. Bertoncini F, Di Berardino S, Lo Burgio I, et al. La ricerca finalizzata in Area Medica: approfondimenti assistenziali dell'Associazione A.N.Í.M.O. Quaderni Ital J Med 2019;7:28-30.

19. Bertoncini F, Di Berardino S, Lo Burgio I, et al. La ricerca finalizzata in Area Medica: approfondimenti assistenziali dell'Associazione A.N.Í.M.O. Quaderni Ital J Med 2019;7:37-8.

20. Seligardi M, Bassi E, Mongardi M. Esiti sensibili alle cure infermieristiche e staffing: le infezioni correlate all'assistenza. Revisione della letteratura. Assist Inferm Ric 2017;36:172-8.

21. Canzan F, Saiani L, Mortari L, Ambrosi E. Quando i pazienti parlano di cura: Uno studio qualitativo fenomenologico. Assist Inferm Ric 2013;32:205-12.

22. Bertoncini F, Di Berardino S, Lo Burgio I, et al. La ricerca finalizzata in Area Medica: approfondimenti assistenziali dell'Associazione A.N.Í.M.O. Quaderni Ital J Med 2019;7:40-2.

23. Palese A, Cargnelli G, Bicego L, et al. Progettare e condurre studi sulla contenzione fisica: appunti di metodo. Assist Inferm Ric 2018;37:76-82.

24. Zanetti E, Zani M, Poli M, et al. La contenzione a domicilio: indagine di prevalenza tra gli utenti del Servizio di Assistenza Domiciliare dell'Azienda ULSS n. 7 Pedemontana, Distretto 1 Bassano del Grappa (VI). Assist Inferm Ric 2018;37:62-7.

25. Jones TL. Outcome measurement in nursing: Imperatives, ideals, history, and challenges. Online J Issues Nurs $2016 ; 21$. 


\section{LINEE GUIDA PER GLI AUTORI}

I Quaderni dell 'Italian Journal of Medicine (Quaderni ITJM), costituiscono una collana supplementare solo online annessa alla rivista Italian Journal of Medicine contenente lavori solo in lingua italiana.

I Quaderni ITJM pubblicano:

- Monografie ad hoc individuate dal Presidente FADOI, dal Consiglio Direttivo, dal Board Scientifico o dall'Editor in Chief dell'Italian Journal of Medicine, in funzione del contesto scientifico-istituzionale attuale.

- Monografie ad hoc su temi di particolare rilevanza scientifica a cura della Commissione FADOI Giovani.

- Traduzioni in italiano di alcuni lavori pubblicati sui numeri standard dell'Italian Journal of Medicine, di particolare interesse per la comunità scientifica.

\section{STESURA DEI LAVORI}

I lavori dovranno essere redatti in modo conforme alle linee guida sotto riportate:

- I manoscritti devono essere scritti interamente in lingua italiana, su documento di Word, con carattere Times New Roman/Arial, dimensione 12, formato A4, interlinea doppia e margini $2,54 \mathrm{~cm}$.

Parole totali: $\max$ 4000; Sommario/Abstract: $\max 250$ parole; Bibliografia: $\min 40$ voci; Tabelle e Figure: $3 / 5$ totali (le tabelle non devono superare n. 1 pagina del documento in Word).

- La strutturazione del contenuto deve attenersi agli standard internazionali per la Rassegna (Review): i) Abstract riassuntivo dell'intero lavoro; ii) Introduzione al tema trattato; iii) Criteri e strumenti di ricerca (criteri di inclusione/esclusione, banche dati consultate, ...); iv) i successivi paragrafi devono illustrare le più recenti scoperte scientifiche nel settore; v) Conclusioni; vi) Bibliografia.

- La prima pagina deve riportare: i) titolo (in stampatello minuscolo), senza acronimi; ii) nome e cognome per esteso di ciascun autore; iii) affiliazione(i) di ciascun autore, numerate con numeri arabi; iv) eventuali ringraziamenti; v) nome e indirizzo postale completi dell'autore corrispondente, corredati da telefono, fax, e-mail; vi) da 3 a 5 parole chiave, separate da virgola. La seconda pagina può riportare: i) contributi degli autori, e.g. informazioni relative a contributi sostanziali delle persone coinvolte nello studio (http://www.icmje.org/\#author); ii) dichiarazione relativa a potenziali conflitti d'interesse; iii) ulteriori informazioni (e.g. fondi, esposizioni durante conferenze...).

- In caso di utilizzo di tabelle, queste devono essere tutte numerate con numeri arabi e citate nel testo in ordine consecutivo (e.g. NON nominare le tabelle come Tabella $1 \mathrm{~A}, 1 \mathrm{~B}, \ldots$ o $1.0,1.1, \ldots$ ). Le tabelle devono essere presentate in formato editabile. Ciascuna tabella deve essere corredata da una breve didascalia; in caso di abbreviazioni, riportare una nota a piè di CIASCUNA tabella che spieghi TUTTE le abbreviazioni presenti in ognuna.

- In caso di utilizzo di figure, queste devono essere inviate in formato tiff o .jpg, allegate al manoscritto in singoli files, secondo le seguenti specifiche:

i) a colori (salvate in modalità CMYK): minimo 300 dpi di risoluzione;

ii) in bianco e nero: minimo 600 dpi di risoluzione;

iii) minimo $17,5 \mathrm{~cm}$ di larghezza.

Ciascuna figura deve essere corredata da una breve didascalia.

$N B$ : In caso di Tabelle/Figure riprese e/o modificate da altri lavori già pubblicati, sarà cura degli autori accertarsi se tali materiali siano o meno coperti da copyright e procurarsi i permessi necessari per la riproduzione. Tali permessi dovranno essere allegati alla versione definitiva del lavoro. L'ufficio editoriale si riserva la facoltà di rimuovere Tabelle/Figure coperte da copyright, se sprovviste dei necessari permessi.

- In caso di utilizzo di abbreviazioni, la prima volta che esse sono citate è necessario scrivere per esteso la definizione+abbreviazione tra parentesi tonde [e.g. risonanza magnetica (RMN)], a seguire si dovrà riportare solo l'abbreviazione (unica eccezione: nei titoli e nelle didascalie di tabelle e figure NON si utilizzano abbreviazioni).

\section{BIBLIOGRAFIA}

Le voci bibliografiche devono essere formattate secondo lo stile Vancouver.

Nella sezione Bibliografia, le voci bibliografiche devono essere numerate consecutivamente nell'ordine in cui appaiono per la prima volta nel testo (NON in ordine alfabetico) e, nel testo, devono essere indicate con numeri arabi in apice. Voci bibliografiche riferite a comunicazioni personali o dati non pubblicati devono essere incorporate nel testo e NON inserite tra le voci numerate [e.g. (Wright 2011, dati non pubblicati) o (Wright 2011, comunicazione personale)]. Le voci bibliografiche nella sezione Bibliografia devono tassativamente essere preparate come segue:

i) più di 3 autori, citare 3 autori, et al. Se il lavoro contiene solo 4 autori, citarli tutti e 4;

ii) titolo del lavoro in stampatello minuscolo;

iii) nome della rivista, senza punti, abbreviato secondo gli standard internazionali; in caso di dubbi sulla corretta abbreviazione, fare riferimento ai seguenti siti:

a. ISI Journal Abbreviations Index (http://library.caltech.edu/ reference/abbreviations/);

b. Biological Journals and Abbreviations (http://home.ncifcrf. gov/research/bja/);

c. Medline List of Journal Titles (ftp://ftp.ncbi.nih.gov/pubmed/J_Medline.txt);

iv) inserire l'anno di pubblicazione subito dopo il nome della rivista, seguito da punto e virgola;

v) NON inserire giorno o mese di pubblicazione;

vi) citare solo il volume, seguito dai due punti (NON citare il fascicolo tra parentesi);

vii) abbreviare le pagine, e.g. 351-8

Per accertarsi di aver correttamente formattato le voci bibliografiche, confrontarle con le citazioni in PubMed (http://www.ncbi. nlm.nih.gov/pubmed).

Esempi (prestare attenzione anche alla punteggiatura):

Articolo standard su Rivista

Halpern SD, Ubel PA, Caplan AL. Solid-organ transplantation in HIV-infected patients. N Engl J Med 2002;347:284-7.

Proceedings

Christensen S, Oppacher F. An analysis of Koza's computational effort statistic for genetic programming. In: Foster JA, Lutton E, Miller J, Ryan C, Tettamanzi AG, eds. Genetic programming. EuroGP 2002: Proceedings of the 5th European Conference on Genetic Programming, 2002 Apr 3-5, Kinsdale, Ireland. Berlin: Springer; 2002. pp 182-91.

Articoli i cui autori sono Organizzazioni

Diabetes Prevention Program Research Group. Hypertension, insulin, and proinsulin in participants with impaired glucose tolerance. Hypertension 2002;40:679-86.

Libri

Murray PR, Rosenthal KS, Kobayashi GS, Pfaller MA. Medical microbiology. 4th ed. St. Louis, MO: Mosby; 2002. (CITAZIONE DEL LIBRO INTERO)

Meltzer PS, Kallioniemi A, Trent JM. Chromosome alterations in human solid tumors. In: Vogelstein B, Kinzler KW, eds. The genetic basis of human cancer. New York, NY: McGraw-Hill; 2002. pp 93113. (CITAZIONE DI UN CAPITOLO)

\section{MODALITÀ D'INVIO DEI LAVORI}

Monografie

Gli Autori dovranno fare riferimento all'Editor-in-Chief o alle persone da lui designate nelle lettere di invito a scrivere gli articoli programmati.

Per gli articoli inviati da giovani internisti, gli Autori dovranno fare riferimento al Dr. Michele Meschi (e-mail: mmeschi@ausl.pr.it)e agli eventuali altri referenti da lei designati, nelle prime fasi di stesura dei manoscritti, revisioni e correzioni. 


\section{QUADERNI}

dell'Italian Journal

of Medicine

Il Dr. Meschi raccoglierà poi le versioni definitive dei lavori di ciascuna monografia e provvederà all'invio di tutti i materiali all'ufficio editoriale.

I lavori solo nella loro versione definitiva e approvata dalla Commissione FADOI Giovani dovranno pervenire all'ufficio editoriale già pronti per l'impaginazione e immediata pubblicazione (già corredati da eventuali permessi per la riproduzione di tabelle e immagini redatti secondo le presenti linee guida).

Traduzioni

Previo invito dell'Editor-in-Chief, gli Autori dovranno far pervenire all'ufficio editoriale la versione tradotta in italiano, al seguente indirizzo e-mail: paola.granata@pagepress.org

Il file in formato Word dovrà essere formattato secondo gli standard editoriali della rivista ufficiale ed essere già pronto per impagina-

\section{STAFF EDITORIALE}

Paola Granata, Journal Manager

paola.granata@pagepress.org

Claudia Castellano, Production Editor

Tiziano Taccini, Technical Support

\section{PUBBLICATO DA}

PAGEPress Publications

via A. Cavagna Sangiuliani 5

27100 Pavia, Italy

T. +39.0382 .464340$

F: +39.0382 .34872$

\section{agepress}

www.pagepress.org info@pagepress.org zione e immediata pubblicazione (corredato da eventuali permessi per la riproduzione di tabelle e immagini).

Si prega di inviare le eventuali tabelle in formato editabile e le figure in alta definizione secondo gli standard sopra riportati.

\section{NOTA PER GLI AUTORI}

I lavori pubblicati sui Quaderni ITJM non verranno indicizzati, ma saranno liberamente disponibili in un'apposita sezione del sito FADOI (http://www.fadoi.org/) e della rivista ufficiale.

Gli Autori i cui lavori siano accettati per la pubblicazione sui Quaderni ITJM e che fossero interessati a vederli pubblicati anche sulla rivista ufficiale, dovranno sottomettere attraverso il sito dell'ITJM (www.italjmed.org) la versione (già tradotta) in inglese e redatta in modo conforme alle linee guida della rivista; seguiranno poi la procedura di selezione tramite peer review e, se accettati, saranno inseriti nel piano editoriale standard.

\section{QUADERNI - ITALIAN JOURNAL OF MEDICINE}

Tutti gli articoli pubblicati sui QUADERNI - Italian Journal of Medicine sono redatti sotto la responsabilità degli Autori. La pubblicazione o la ristampa degli articoli della rivista deve essere autorizzata per iscritto dall'editore. Ai sensi dell'art. 13 del D.Lgs 196/03, i dati di tutti i lettori saranno trattati sia manualmente, sia con strumenti informatici e saranno utilizzati per l'invio di questa e di altre pubblicazioni e di materiale informativo e promozionale. Le modalità di trattamento saranno conformi a quanto previsto dall'art. 11 del D.Lgs 196/03. I dati potranno essere comunicati a soggetti con i quali PAGEPress intrattiene rapporti contrattuali necessari per l'invio delle copie della rivista. Il titolare del trattamento dei dati è PAGEPress Srl, via A. Cavagna Sangiuliani 5 - 27100 Pavia, al quale il lettore si potrà rivolgere per chiedere l'aggiornamento, l'integrazione, la cancellazione e ogni altra operazione di cui all'art. 7 del D.Lgs 196/03.

https://www.italjmed.org/index.php/ijm/quad 
PAGEPress - Scientific Publications

Via A. Cavagna Sangiuliani, 5 - 27100 Pavia - Italy

Phone +390382 464340 - Fax +39038234872

www.pagepress.org 